\title{
The impact of oral anticoagulants on haemostasis
}

Citation for published version (APA):

Zwaveling, S. (2019). The impact of oral anticoagulants on haemostasis: a step towards individualized therapy. [Doctoral Thesis, Maastricht University]. Ridderprint BV. https://doi.org/10.26481/dis.20190503sz

Document status and date:

Published: 01/01/2019

DOI:

10.26481/dis.20190503sz

Document Version:

Publisher's PDF, also known as Version of record

\section{Please check the document version of this publication:}

- A submitted manuscript is the version of the article upon submission and before peer-review. There can be important differences between the submitted version and the official published version of record.

People interested in the research are advised to contact the author for the final version of the publication, or visit the DOI to the publisher's website.

- The final author version and the galley proof are versions of the publication after peer review.

- The final published version features the final layout of the paper including the volume, issue and page numbers.

Link to publication

\footnotetext{
General rights rights.

- You may freely distribute the URL identifying the publication in the public portal. please follow below link for the End User Agreement:

www.umlib.nl/taverne-license

Take down policy

If you believe that this document breaches copyright please contact us at:

repository@maastrichtuniversity.nl

providing details and we will investigate your claim.
}

Copyright and moral rights for the publications made accessible in the public portal are retained by the authors and/or other copyright owners and it is a condition of accessing publications that users recognise and abide by the legal requirements associated with these

- Users may download and print one copy of any publication from the public portal for the purpose of private study or research.

- You may not further distribute the material or use it for any profit-making activity or commercial gain

If the publication is distributed under the terms of Article $25 \mathrm{fa}$ of the Dutch Copyright Act, indicated by the "Taverne" license above, 


\section{THE IMPACT OF \\ ORAL ANTICOAGULANTS \\ ON HAEMOSTASIS}

A STEP TOWARDS INDIVIDUALIZED THERAPY

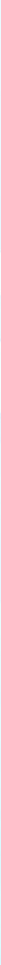

SUZANNE ZWAVELING 

THE IMPACT OF ORAL

ANTICOAGULANTS ON HAEMOSTASIS

A STEP TOWARDS INDIVIDUALIZED THERAPY

Suzanne Zwaveling 
(C) Suzanne Zwaveling, Maastricht 2019

All rights are reserved. No part of this book may be reproduced, stored in a retrieval system or transmitted in any form or by any means, without prior permission in writing from the author or, where appropriate, the publisher of the article.

Cover design: Sophia Zwaveling, Michael Laurens

Layout: $\quad$ Jules Verkade, persoonlijkproefschrift.nl

Printing: $\quad$ Ridderprint BV | www.ridderprint.nl

ISBN: $\quad$ 978-94-6375-299-2

Financial support by Stichting Hart Onderzoek Nederland (www.hartonderzoek.nu), is gratefully acknowledged.

Financial support for printing this thesis was kindly provided by the department of biochemistry of Maastricht University and Stago BNL.

Financial support by the Dutch Heart Foundation for the publication of this thesis is gratefully acknowledged. 


\title{
The impact of oral anticoagulants on haemostasis
}

\author{
A step towards individualized therapy
}

\author{
PROEFSCHRIFT
}

ter verkrijging van de graad van doctor aan de Universiteit Maastricht, op gezag van de Rector Magnificus, Prof. dr. Rianne M. Letschert, volgens het besluit van het College van Decanen,

in het openbaar te verdedigen

op vrijdag 3 mei 2019 om 12:00 uur

door

Suzanne Zwaveling

Geboren op 22 mei 1988 te Utrecht 


\section{Promotor:}

Prof. dr. H. ten Cate

\section{Copromotores:}

Dr. S. Bloemen

Dr. A. ten Cate-Hoek

\section{Beoordelingscommissie:}

Prof. dr. T. M. Hackeng (voorzitter)

Prof. dr. R.P. Koopmans

Dr. K. Winckers

Prof. dr. R.A.S. Ariëns (University of Leeds)

Prof. dr. R.E.G. Schutgens (Universiteit Utrecht) 


\section{CONTENTS}

Chapter $1 \quad$ General introduction and outline of this thesis 7

Chapter $2 \quad$ Prediction of bleeding risk in patients taking vitamin $\mathrm{K} \quad 31$ antagonists using thrombin generation testing

Chapter 3 Calibrated automated thrombinography (CAT), a tool to identify patients at risk of bleeding during anticoagulant, therapy? A systematic review

Chapter $4 \quad$ The anticoagulant effect of dabigatran is reflected in the lag time and time-to-peak, but not in the endogenous thrombin potential or peak, of thrombin generation

Chapter 5 A new assay for the concomitant assessment of rivaroxaban concentration and its impact on thrombin generation

Chapter 6 The variability in response to rivaroxaban and apixaban between and within individuals, over time

Chapter 7 The differential effects of dabigatran, rivaroxaban and apixaban on fibrinolysis

Chapter 8 Summary, general discussion and future prospects 163

Chapter $9 \quad$ Nederlandse samenvatting

Appendix I Valorisation

$\begin{array}{lll}\text { Appendix II About the author } & 200\end{array}$

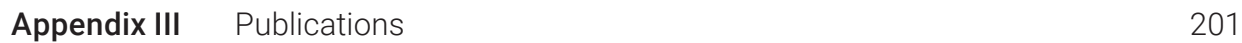

Appendix IV Dankwoord 204 


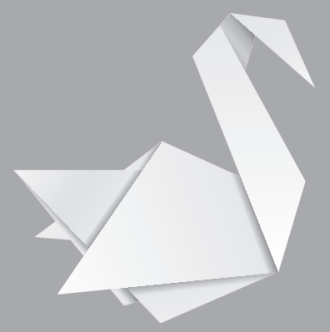




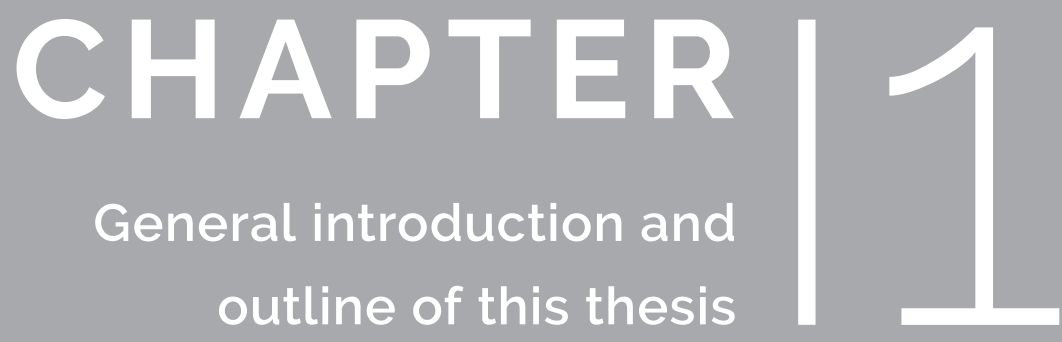




\section{HAEMOSTASIS - PHYSIOLOGY}

The physiological response to vessel injury by the formation of a clot to maintain adequate blood flow in the vessel under any circumstance is called haemostasis. The maintenance of haemostasis is achieved by three complementary systems: blood platelets, coagulation and fibrinolysis. ${ }^{1-3}$ When vessel injury occurs multiple important mechanisms are initiated in parallel. The vessel constricts to reduce the injured surface and collagen is displayed from the wound, which directly activates platelets. By physiologic rules of flow, platelets are pushed outwards, to the proximity of the vessel wall, by the bloodstream. The endothelial adhesive protein von Willebrand Factor (VWF), helps to trap platelets and adhere them onto the damaged endothelial wall, in a shear dependent mechanism. Subsequently, the activation of platelets by thrombin and other mediators stimulates the recruitment and activation of more platelets, forming a haemostatic plug. ${ }^{3}$

At the same time, a chain-reaction of enzymes is started, to finally form a fibrin network, which stabilizes the primary haemostatic clot. This reaction, in which enzymes rapidly activate one another, is known as coagulation. "Ex vivo" in the laboratory, coagulation can be initiated via two main routes traditionally known as the extrinsic and the intrinsic pathway. In reality these routes do not operate separately but are intertwined via feedback loops, to facilitate the formation of the pivotal enzyme thrombin. ${ }^{4}$ Thrombin cleaves fibrinogen into fibrin and is therefore directly responsible for producing the actual clot. In vivo, upon damage of the vascular endothelium, coagulation is started by the release of tissue factor (TF) from the site of injury, originally described as the "extrinsic pathway", presented in figure 1a. TF forms a complex with activated factor VII (FVIIa), the TF/FVIIa complex, which then activates FX. Subsequently, FXa can convert prothrombin (FII) into thrombin (FIla). These initial thrombin molecules stimulate further thrombin production by several positive feedback mechanisms. The most important feedback loop is the activation of factor $\mathrm{V}$ into $\mathrm{FVa}$, by which thrombin enables the formation of the prothrombinase complex (FVa together with FXa and calcium, on a phospholipid surface). When prothrombin binds to the phospholipid surface, the prothrombinase complex can convert prothrombin more efficiently. ${ }^{5,6}$ This is the most important and effective pathway that is responsible for the production of $\mathrm{FXa}$, and thereby thrombin, in the presence of high TF concentrations. To make sure that sufficient amounts of thrombin can be formed even at low concentrations of TF, another feedback loop can be initiated by the first trace amount of thrombin. ${ }^{7}$ Thrombin activates FVIII and 
FXI. FVIIla is a cofactor for factor FIXa, which together form the intrinsic "tenase complex" on a phospholipid surface. This complex can be formed upon activation by either factor FXIa (via the originally described intrinsic route), or by low concentrations of the TF/FVIla complex (via the extrinsic route), as shown in figure 1a. The tenase complex enhances the generation of FXa. This feedback loop is known as the Josso Loop and can boost thrombin generation up to 95\% (Figure 1). 8,9 Ultimately, thrombin activates FXIII, thereby stimulating the formation of crosslinks between fibrin fibres, which results in a more stable clot. ${ }^{10}$

Ex vivo, a second pathway, formerly known as the "intrinsic route" and today as the contact activation pathway, can also initiate coagulation. Via this route, FXIla activates FXI (FXIa then activates FIX as described above) and prekallikrein in the presence of high molecular weight kininogen (HMWK). Prekallikrein can boost the formation of FXIla via the activation of kallikrein. Although this mechanism is well established in vitro, the physiologic functions of the contact system in haemostasis are still uncertain.

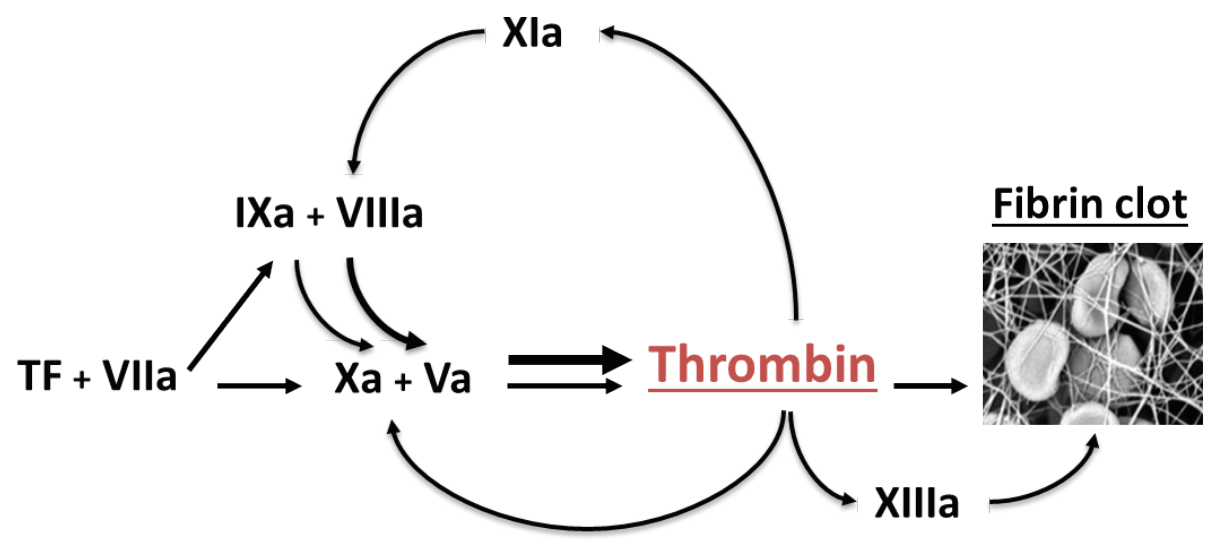

Figure 1. The extrinsic pathway and the feedback loops generating thrombin.

In addition to its major role in coagulation, thrombin is also a potent platelet activator, mostly by stimulating protease-activated receptor (PAR)-1 and, at high concentrations, PAR-4. The increased aggregation by activated platelets aids coagulation by providing a phospholipid surface. Inactivated platelets carry negatively charged phospholipids (PL) such as phosphatidylserine (PS) on the inside of their membrane. Activation of platelets causes transmembrane translocation of PL, a flip-flop mechanism, in which PS is externalized. This creates a negatively charged surface that facilitates adherence of positively charged coagulation factors. 
Besides enabling its own production, thrombin also inhibits its own generation via the activated protein C (APC) system, as is shown in figure 2. Thrombomodulin (TM) binds thrombin at exosite 1, forming a complex that promotes the activation of protein C into APC. Supported by protein S, APC can inactivate FVa. ${ }^{11}$ Additionally, protein S and FVa function as synergetic cofactors in the APC-mediated inactivation of FVIIIa. ${ }^{12}$ Thus, by tempering two positive feedback loops, thrombin generation is dampened. To further prevent excess clotting, i.e. thrombus formation, several other natural anticoagulant systems are operational: firstly, antithrombin (AT) is a serine protease inhibitor that binds and inactivates multiple coagulation enzymes, but most importantly thrombin. Some anticoagulant drugs (heparins) operate by enhancing the activity of AT, as we will discuss later. Secondly, the extrinsic pathway can be inhibited by tissue factor pathway inhibitor (TFPI), a protein which consists of three consecutive Kunitz domains. Kunitz domain 1 can bind to FVIla and the Kunitz 2 can bind to FXa. The function of the third domain is not yet fully understood. TFPI downregulates TF activity by the formation of an inactive complex, in which FXa is bound to the Kunitz 2 domain, and TF/VIla is then connected to the Kunitz 1 domain. Protein S acts as cofactor in the bimolecular complex formation of TPFI with FXa, thereby accelerating the inhibition of FXa by 10 -fold..$^{13}$

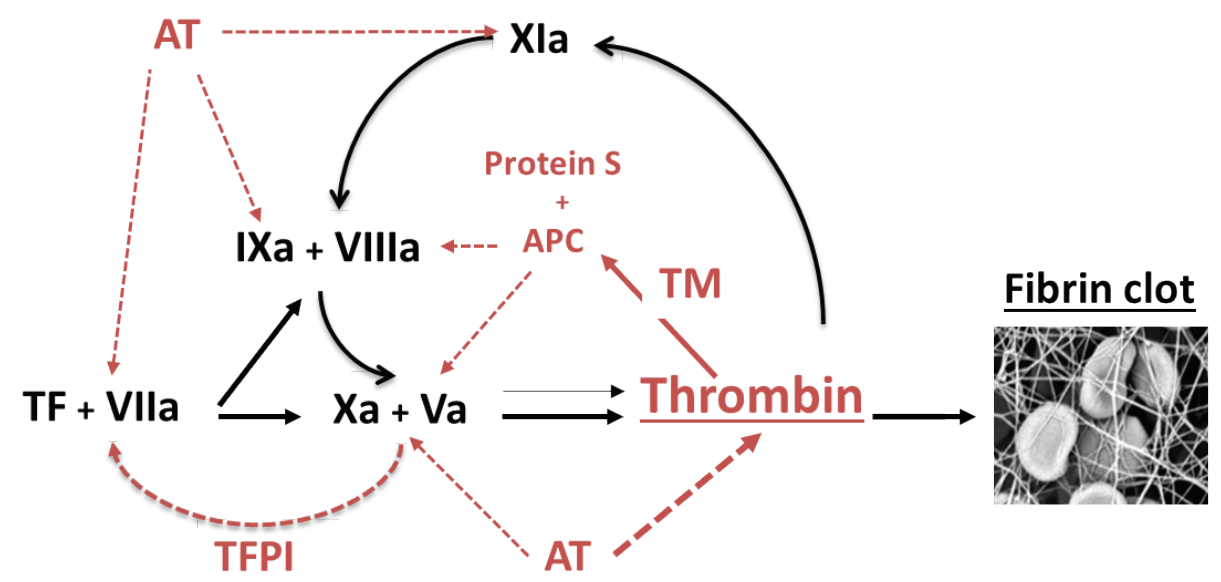

Figure 2. The downregulation of thrombin via multiple pathways. 


\section{FIBRINOLYSIS}

To maintain a balance in haemostasis, the degradation of the fibrin clot is as important as its formation. Degradation of the clot is called fibrinolysis and is summarized in figure 3. Fibrinolysis is started by plasminogen and tissue plasminogen activator (tPA) that bind together to the carboxy-lysine ends of the fibrin molecules in the clot. By the binding to fibrin, tPA can more efficiently convert plasminogen into plasmin. ${ }^{14}$ Plasmin cleaves fibrin into fibrin degradation products (FDPs), which leads to the actual lysis of the clot. In vivo, plasminogen is mainly activated by tPA, released from endothelial cells, but can also be activated by urokinase plasminogen activator (UPA), that is secreted from macrophages, renal epithelium and endothelial cells. Fibrinolysis is modulated through several inhibitors. The most important inhibitors would be plasminogen activator inhibitors 1 and 2 (PAI-1 and PAI-2) that directly inactivate IPA and UPA by incorporating them in a stable, inactive complex. The second halt on fibrinolysis is the direct inhibition of plasmin by $\mathrm{a}_{2}$-antiplasmin.

Besides an important function in clot formation, thrombin plays an essential part in fibrinolysis by controlling several clot protective mechanisms. Firstly, higher concentrations of thrombin are related to thinner fibrin fibres, that promote the formation of a more compact and denser network. ${ }^{15}$ Secondly, thrombin activates factor XIII, which enhances clot strength and links $\mathrm{a}_{2}$-antiplasmin to fibrin supporting the inactivation of plasmin. ${ }^{10}$ Finally, thrombin in complex with TM, activates thrombin-activatable fibrinolysis inhibitor (TAFI). Activated TAFI (TAFla) protects the clot against fibrinolysis by removing the binding sites for tPA and plasminogen of the fibrin fibres, limiting plasmin generation. ${ }^{16,17}$ Fibrinolysis can be measured by monitoring the changes in turbidity during clot formation and subsequent degradation, using a turbidimetric assay. In this assay, coagulation is initiated by TF. The reaction is started by recalcification of citrated plasma. Simultaneously, exogenous tPA is added to induce clot lysis. The clot lysis time (CLT) is commonly defined as the time from half maximal fibrin formation to half maximal degradation, as displayed in figure 4. This assay is considered a well-established method to study the overall plasma fibrinolytic potential, including effects of plasminogen, $a_{2}$-antiplasmin, PAI-1 and AT.18 


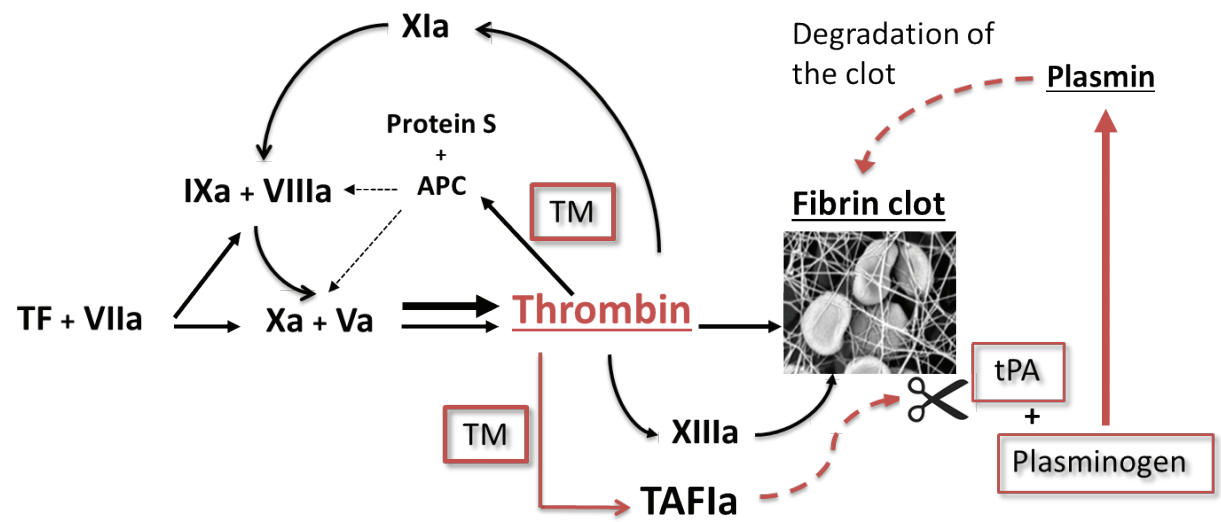

Figure 3. Fibrinolysis and the protective roles of thrombin.

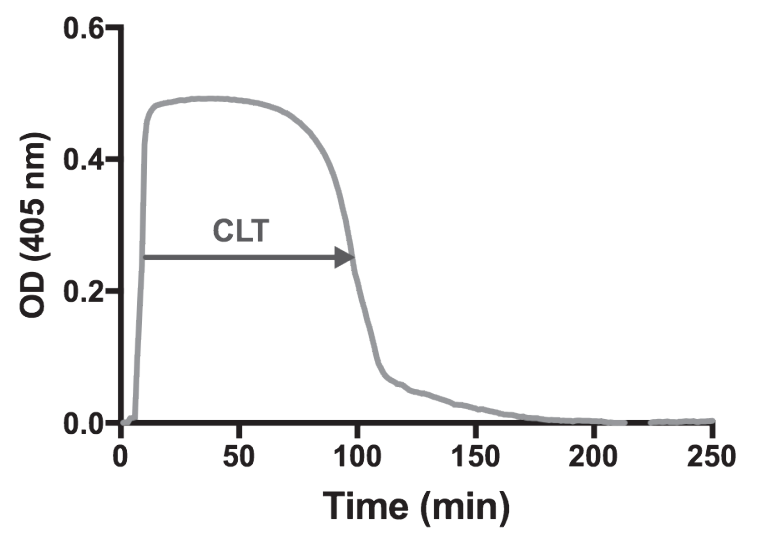

Figure 4. Definition of the clot lysis time (CLT).

The CLT is commonly defined as the time from half maximal fibrin formation (upward line) to half maximal degradation (downward line) in a measurement of the optical density (OD)

\section{HAEMOSTASIS - PATHOLOGY}

\section{Thrombotic disorders}

Under physiological conditions a delicate equilibrium exists between a hyper- and hypocoagulable state. Coagulation is of vital importance to maintain haemostasis and an adequate blood flow in case of severe bleeding. However, the pathological formation of a clot due to hypercoagulation, thrombosis, can be life threatening. 
Thrombosis is caused by an imbalance between three components that are summarized in Virchow's triad: blood flow, the vascular endothelium and coagulation proteins (stimulators and inhibitors). ${ }^{19}$ Thrombosis can occur in arteries (arterial thrombosis) or in veins (venous thrombosis). Together, arterial and venous thrombosis, underlie the most prevalent causes of morbidity and mortality in the Western world, ie myocardial infarction, ischemic stroke and venous thromboembolism (VTE). Currently, thrombosis is the leading cause of death in women. ${ }^{20}$ In the Netherlands 39,128 patients died of cardiovascular disease in the year 2013 , of those 20,815 were women and 18,313 were men. Most of these deaths were caused by arterial thrombosis. ${ }^{21}$

Arterial thrombosis often occurs after the rupture or erosion of an atherosclerotic lesion, which promotes clotting through collagen and TF-mediated mechanisms. The most critical forms of arterial thrombosis occur in the event of an occlusion of one or more main blood vessels to the brain (ischaemic stroke) or heart (myocardial infarction (MI)). In these instances of atherothrombosis, platelets play an important role in the pathophysiology and primary antithrombotic management consists of platelet inhibitors. Moreover, in case of an acute arterial thrombus, arterial stenting via a percutaneous coronary intervention (PCI) can be warranted. After the insertion of a stent, the foreign material is highly coagulable and the combined action of two platelet inhibitors is required for 6 months or 1 year, depending on the cause for $\mathrm{PCl}$, to prevent recurrent myocardial ischemia. In case of an ischemic stroke, thrombolysis and/or intra-arterial thrombectomy, are presently first line interventions, followed by platelet inhibitors to prevent recurrence.

Venous thrombosis can occur in deep or more superficial veins, due to triggering of coagulation by altered flow conditions and inflamed endothelium. In VTE hypercoagulability is dominant over platelet activation in driving thrombosis. Hence, anticoagulant therapy is the intervention of choice in case of acute VTE. The incidence of VTE in the Netherlands is about 1-2 per 1000 persons per year. Annually 1250 patients die of VTE. The most feared acute complication of venous thrombosis is pulmonary embolism (PE), the result of incomplete clot breakdown, producing emboli that pass through the heart and end up in the pulmonary circulation. When patients suffer haemodynamic instability, thrombolytic therapy may sometimes be warranted in PE, in addition to regular anticoagulation therapy. Acute VTE will be treated with oral anticoagulant therapy for at least three months and longer, depending on additional thrombotic risk factors. 
A common disorder that deserves special mention because it is highly associated with hypercoagulability is atrial fibrillation. Atrial fibrillation (AF) is the most common rhythm disorder of the heart. In AF, the atria of the heart do not respond to the regular electrical conduction directed by the sinoatrial (SA) node, but contract irregularly. The percentage of patients with AF increases with age, with $0.1 \%$ of the patients under 50 and $14 \%$ of the patients over 80 years old, being affected. ${ }^{22}$ Because the atria "quiver" more than they contract functionally, blood coagulation may be activated locally resulting in thrombus formation in the atria, particularly in the left atrial appendage. Smaller emboli can detach from such a thrombus and circulate with the bloodstream through the left chamber into the arteries to the brain, causing an ischemic stroke. Consequently, all patients with AF are screened with the $\mathrm{CHA}_{2} \mathrm{DS}_{2}-\mathrm{VASc}$ score, to assess their individual risk of stroke. A score of 0 in males and 1 in females is considered a low risk of stroke. A score of 1 in males or above 1 in females is considered an increased risk of stroke (>1-2\%) and these patients should receive oral anticoagulants for an indefinite period of time. ${ }^{23}$

\section{ANTICOAGULANT TREATMENT}

Over the last few years more anticoagulant drugs have become available. This increase in available anticoagulants and platelet inhibitors has further improved, but also complicated treatment. Physicians have more choice between different anticoagulant drugs than a few years ago. Moreover, each drug has its own prescription regimen, indications, contra-indications and interactions, which have to be taken into account to prevent adverse drug events (ADE). Sometimes treatment with anticoagulants and platelet inhibitors has to be combined, which increases the risk of bleeding even more. ${ }^{24-26}$ Several studies have shown that visits to the emergency department because of ADEs most commonly involve antithrombotic medication. ${ }^{27}$ Moreover, anticoagulants are among the most common drugs that cause ADEs in hospitalized patients..$^{28}$ An important message from these studies is that most ADEs from anticoagulant drugs result from human errors, and are therefore potentially preventable.

\section{Parenteral treatment}

In prophylaxis of VTE, low molecular weight heparins (LMWHs) are the most commonly used drugs. LMWHs are also being used to treat VTE in patients with cancer, or during pregnancy. The anticoagulant effect of LMWHs is based on the functional enhancement of 
AT. The binding of LWMHs to AT causes a conformational change in the protease inhibitor, that accelerates the inactivation rate of (primarily) FXa by AT. The length of the sugar-chain of the LMWH molecule determines whether AT inactivates just FXa, or Flla as well. Although the LMWHs are efficient drugs, one important disadvantage is the route of administration, which is by subcutaneous injection. Moreover, there is relatively poor information about the plasma activity of LMWH in relation to outcome (prevention of (recurrent) thromboembolism, or bleeding). LMWH activity can be measured by determining the anti-FXa concentration, but evidence on how well these levels correlate with clinical outcome is non-conclusive. Therefore, in general, monitoring guided dose-adjustment is not recommended and only advised in certain situations e.g. during pregnancy or in case of extreme body weight. Two other types of heparins are used in current clinical practice: unfractionated heparin (UFH), which is mainly used during cardio-pulmonary bypass surgery and synthetic pentasaccharides (e.g. fondaparinux). Both drugs act via AT, respectively on FXa and Flla or FXa alone, respectively; further elaboration on these drugs is beyond the scope of this thesis.

\section{Oral anticoagulant therapy - Vitamin K antagonists}

The treatment of thrombosis relies on oral anticoagulant drugs. For many years, vitamin $\mathrm{K}$ antagonists (VKAs) have been the cornerstone in oral anticoagulation treatment. VKAs are prescribed for the prevention of thrombosis in patients with AF, and as treatment and secondary prevention in patients with VTE. Vitamin $\mathrm{K}$ is needed for the production of functionally active FII, FVII, FIX and FX, as well as protein C and protein S. VKAs (warfarin, acenocoumarol and phenprocoumon) do not inhibit vitamin $\mathrm{K}$ directly, as the name would suggest, but inhibit vitamin $\mathrm{K}$ epoxide reductase. This enzyme regulates the recycling of inactive vitamin $\mathrm{K}$ into active vitamin $\mathrm{K}$. By inducing vitamin $\mathrm{K}$ depletion, these drugs inhibit the production of four clotting factors. The onset of anticoagulant effect of these drugs takes several days, which makes bridging therapy with fast acting anticoagulants like LMWH necessary. The anticoagulant effect can remain present for several days to weeks depending on the agent's half-life time. VKAs are easily influenced by food or drugs. Consequently, the dosing regimen is not straightforward. The effect of VKAs can be measured with the prothrombin time (PT), a standardized laboratory assay, which measures the time until the clot is formed i.e. until the first traces of thrombin are present. Coagulation is triggered with a high concentration (> 100 pM) of TF. Due to the excess amount of TF the PT gives a fast result (on average 10s), but only provides information on the extrinsic pathway. This clotting time-based assay is standardized to the international normalized ratio (INR) to be 
comparable between different facilities, with a value of 1.0 representing a normal clotting time ratio. The intention of anticoagulation with VKAs is to achieve prolongation of the clotting time with an INR value between 2.0 and 3.0, the "sweet spot" for most indications, as shown in figure 5. INR values below 2.0 are associated with an increased risk of thrombosis, an INR above 3.0 is associated with an increased risk of bleeding. ${ }^{29}$ The expected incidence of bleeding per year during long-term treatment with VKAs is about $10 \%-17 \%$ for all types of bleeding, $2 \%-5 \%$ for major bleeding, and $0.5 \%-1 \%$ for fatal bleeding. The occurrence of intracranial haemorrhage $(\mathrm{ICH})$, which is the most feared bleeding complication, is rare (0.2\%-0.4\% per year). ${ }^{30}$ Based on practical limitations, including laboratory monitoring and the need for repeated dose adjustments, there has been a long search for oral anticoagulants that would require less intensive management.

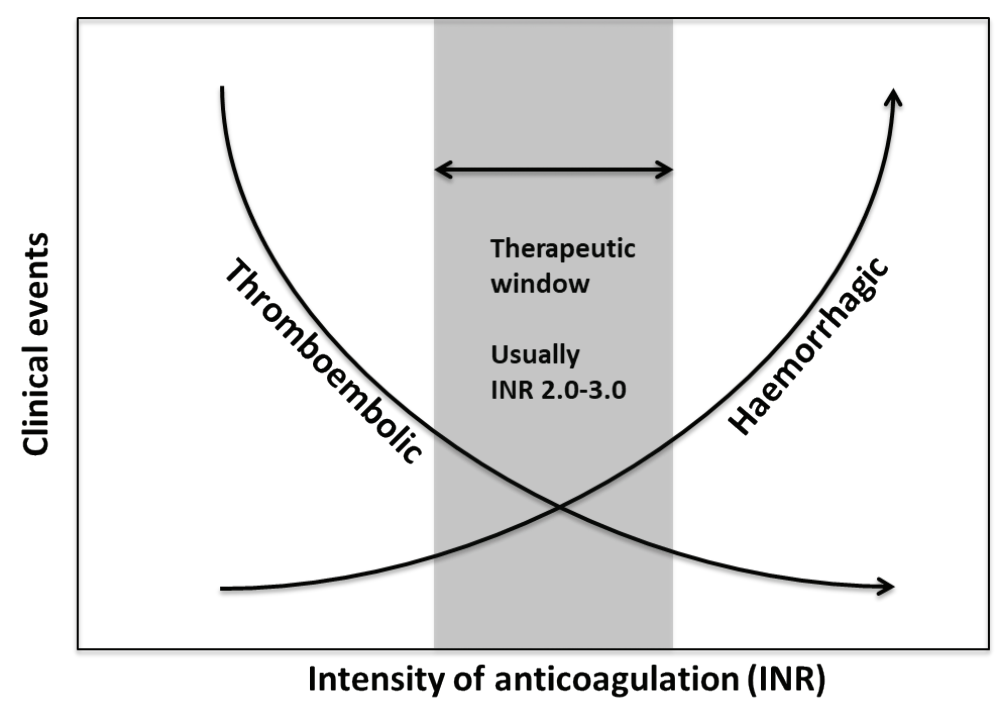

Figure 5. The therapeutic window for patients treated with VKAs. Figure adapted from Blann et al. ${ }^{29}$

\section{Oral anticoagulant therapy - Direct oral anticoagulants}

Since 2012, four direct oral anticoagulants (DOACs) have been registered for clinical use. All DOACs are selective, reversible, small molecular inhibitors of one specific coagulation factor (Flla or FXa). Dabigatran is a direct thrombin inhibitor, whereas rivaroxaban, apixaban and edoxaban are all direct FXa inhibitors. For this thesis it is important to note that these drugs are small molecules that can inhibit their target factor even if these factors are incorporated in a complex. For example, direct FXa inhibitors can inhibit free FXa, but also FXa within 
the prothrombinase complex. Large randomized non-inferiority trials that compared these drugs to VKAs, have established both their efficacy and safety to prevent stroke or systemic embolism in patients with non-valvular AF as well to treat symptomatic venous thrombosis. ${ }^{31-38}$ One of the advantages over VKAs in those studies was that less $\mathrm{ICH}$ occurred in the DOAC groups: $0.5 \%{ }^{32}$ for rivaroxaban and $0.12 \%^{31}$ for dabigatran. However, DOACs still show $14.9 \%$ clinically relevant bleedings per year. ${ }^{32}$ Similar major bleeding rates were reported in these trials comparing warfarin with rivaroxaban (3.45\% vs. $3.6 \%$ ) or with dabigatran $3.4 \%$ vs. $3.1 \%$ in patients with non-valvular AF during a 2-year follow-up. ${ }^{31,32}$ Only apixaban and edoxaban, in comparison to warfarin, showed a significantly lower rate of major bleeding in patients with non-valvular AF (2.1\% vs. $3.1 \%$ and $2,75 \%$ vs. $3,43 \%) .33,34$

Since 2016 DOACs have become the first choice of anticoagulants for the prevention of ischemic stroke in patients with AF and for the treatment of VTE. In addition, all DOACs, with the exception of edoxaban, can be used as thrombo- prophylaxis around knee- or hip replacement surgery. Low dose rivaroxaban exclusively, is approved for the prevention of major adverse events after acute coronary syndrome (ACS) in combination with antiplatelet therapy. ${ }^{39}$ The major advantage over VKAs, and hence the main selling point of DOACs, is their proclaimed pharmacokinetic and -dynamic stability, in part due to fewer interactions with food or drugs. Therefore, they are typically administered in a fixed dose, adjusted only to clinical criteria (body weight, age, renal function etc., illustrated in table 1). Routine laboratory monitoring is not recommended, however, there are multiple clinical scenarios in which insight in DOAC levels and their effect on coagulation could have clinically relevant consequences. These scenarios include: life-threatening bleeding or suspected thromboembolism under DOAC treatment; assessment of safety to perform acute interventions, in particular when associated with high bleeding risk; decision on the use of thrombolytic therapy in ischemic stroke and suspected drug accumulation (either because of an overdose or due to impaired drug clearance i.e. chronic kidney or liver disease). Although, still under debate, there is a growing consensus that measuring levels in these scenarios could be helpful. ${ }^{40-42}$ 


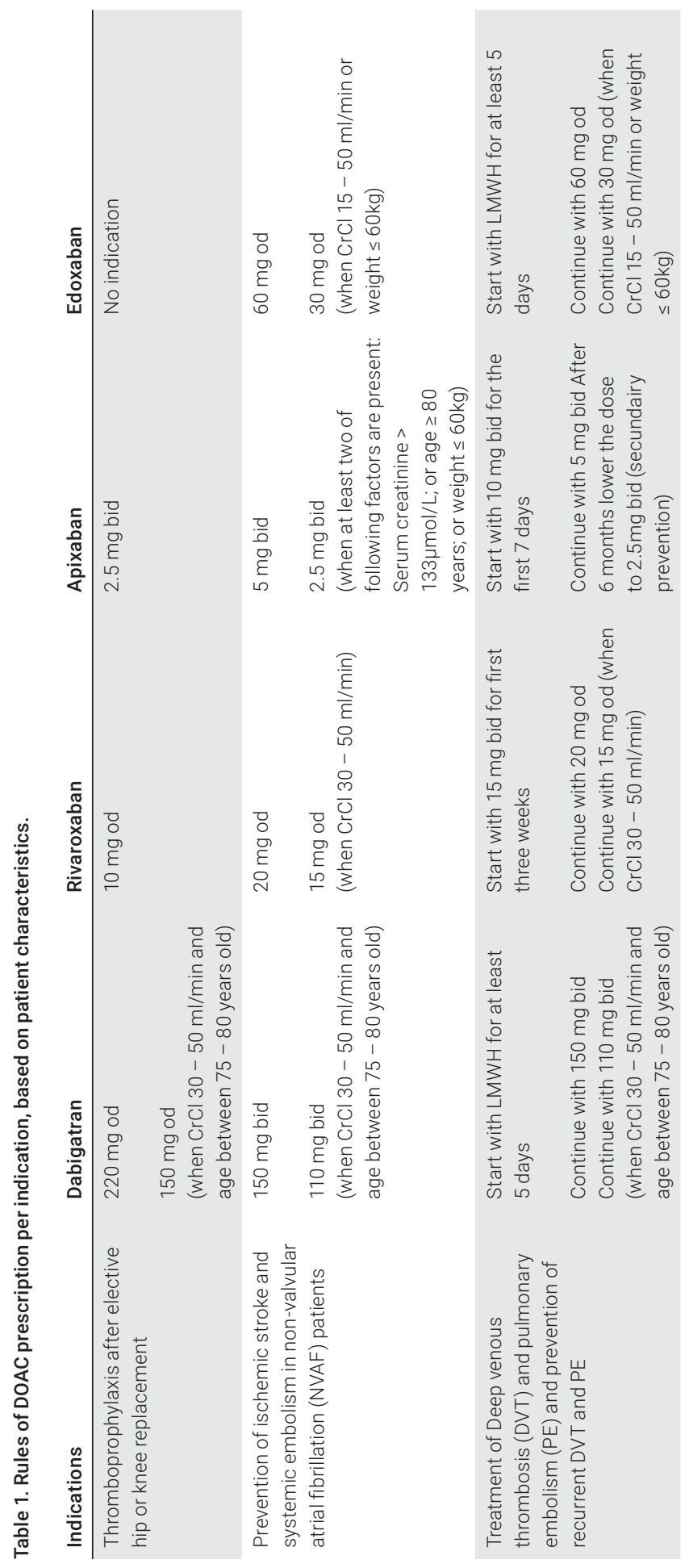


On a personal note, I have encountered the following situations in which the usefulness of the measurement of DOAC levels was evident:

A 65-year-old patient, using dabigatran 150 mg twice daily, was awaiting surgery. Routine coagulation lab results showed a prolonged thrombin time (TT) although the patient had not taken dabigatran the day before surgery. When measured, dabigatran levels proved to be $<30$ $\mathrm{ng} / \mathrm{ml}$, which is considered safe to undergo surgery. ${ }^{43}$ The patient underwent surgery that day without any bleeding complications.

A 76-year-old woman was taking rivaroxaban $20 \mathrm{mg}$ once daily because of a VTE. During treatment she was admitted with a recurrent DVT. Physicians suspected non-adherence, until drug levels were assessed. A level above $600 \mathrm{ng} / \mathrm{m} /$ was found (measured by an anti-FXa assay), which is much above the expected "on-therapy" target value, as can be concluded from table 2. This proved that the patient was taking the drug. This patient was consequently, switched to another DOAC. It could be argued that in this case a functional test should be performed to assess whether the new DOAC does inhibit coagulation sufficiently.

A 95-year-old patient came to the outpatient clinic one month after a percutaneous coronary intervention (PCI) because of myocardial infarction. As comorbidity she had mild renal insufficiency. To prevent stent occlusion she was on antiplatelet therapy (clopidogrel). During her hospital stay, she was also diagnosed with AF, for which apixaban was started in the lowest registered dose (2.5 mg twice daily), according to age and renal function. During consultation she mentioned that she had been suffering from diarrhea since a few days and was not feeling well. Her creatinine level had increased to $150 \mu \mathrm{mol} / \mathrm{L}$ and the estimated clearance was decreased to an estimated glomerular filtration rate (eGFR) of $28 \mathrm{~mL} / \mathrm{min}$, probably due to dehydration. Because of a high bleeding risk during the combined antithrombotic therapy in an old patient with impaired renal clearance, drug levels were measured. Although she had taken the drug 6 hours before blood was drawn, the apixaban level (352 ng/ml) was well above the "on-therapy" peak value (table 2), which should be reached 2-4 hours after intake. The PT and APTT were within the normal range. Switching from DOAC to VKA was recommended, as it was assumed that switching to another DOAC would not lead to lower drug levels, given the observed drug accumulation and the decreased kidney function. This case stresses the importance to consider more frequent follow-up in patients with impaired kidney function. Moreover, it demonstrates that a normal PT and APTT do not exclude the presence of relevant drug levels of apixaban (table 3). ${ }^{44}$ Finally, although a therapeutic range has not been established for DOACs, recent studies 
demonstrate that levels in extreme ranges (too low, or too high, compared to the published "on therapy" levels) are associated with clinical outcomes as bleeding or thrombosis. ${ }^{45-47}$ Since several studies have found large inter-individual variation in drug levels it could be argued that a functional assay would give additional information on this patient's individual coagulation status.

A 72-year-old patient had been admitted to the ICU for over three months. During admission he had suffered from two acute myocardial infarctions for which he underwent $\mathrm{PCl}$ and received 3 drug-eluting stents in total; he was treated with aspirin and clopidogrel. Because of concurrent AF he was using rivaroxaban 20 mg once daily as well. The moment he visited the outpatient clinic he had been using triple therapy for over 3 months, despite having multiple risk factors for an increased risk of bleeding (anaemia, a history of major bleeding, weight loss during admission). Currently he had multiple spontaneous hematomas over his body and reported several nosebleeds. He mentioned that he had not taken any medication on the day of his visit. His lab results showed a PT of $16.7 \mathrm{sec}$ an INR of 1.56 and an aPTT of $37 \mathrm{sec}$. Rivaroxaban levels were high $(386 \mathrm{ng} / \mathrm{ml})$ over 12 hours after last dose intake. It was decided to stop aspirin directly, because 3 months of triple therapy after $\mathrm{PCl}$ is considered sufficient in patients with a high risk of bleeding. Furthermore, rivaroxaban was reduced to $15 \mathrm{mg}$ and continued in combination with clopidogrel for one year, in accordance with results of the PIONEER AF-PCI study. ${ }^{48}$ We advised to measure DOAC levels again after 3 months, to ascertain the appropriateness of this strategy.

Table 2. DOAC dosage and their expected levels. ${ }^{49-52}$

\begin{tabular}{|c|c|c|c|c|c|c|}
\hline \multirow[b]{2}{*}{ Substance } & \multirow[b]{2}{*}{ Dose } & \multicolumn{2}{|c|}{ "on therapy range" (ng/ml) } & \multicolumn{2}{|c|}{$\begin{array}{l}\text { Observed concentrations } \\
\text { at overdose }(\mathrm{ng} / \mathrm{ml})\end{array}$} & \multirow[b]{2}{*}{ Clearance } \\
\hline & & $\begin{array}{l}\text { Trough levels } \\
(12 / 24 \mathrm{~h})\end{array}$ & $\begin{array}{l}\text { Peak Levels } \\
(2-4 \mathrm{~h})\end{array}$ & $\begin{array}{l}\text { Trough levels } \\
(12 / 24 \mathrm{~h})\end{array}$ & $\begin{array}{l}\text { Peak Levels } \\
(2-4 \mathrm{~h})\end{array}$ & \\
\hline \multirow[t]{3}{*}{ Dabigatran } & $150 \mathrm{mg}$ bid & $<30-100$ & $67-356$ & $>200$ & $>450$ & \multirow{3}{*}{$\begin{array}{l}80 \% \text { renal } \\
20 \% \text { faecal }\end{array}$} \\
\hline & $110 \mathrm{mg}$ bid & $40-150$ & $80-300$ & $>200$ & $>450$ & \\
\hline & $220 \mathrm{mg}$ od & $10-100$ & $30-450$ & $>67$ & $>450$ & \\
\hline \multirow[t]{2}{*}{ Rivaroxaban } & $10 \mathrm{mg}$ od & $0-100$ & $50-300$ & $>100$ & $>300$ & \multirow{2}{*}{$\begin{array}{l}33 \% \text { renal } \\
66 \% \text { meta-bolized: } \\
\text { (50\% renal and } \\
\text { other half by } \\
\text { hepatobiliary route) }\end{array}$} \\
\hline & $20 \mathrm{mg}$ od & $5-100$ & $150-300$ & $>120$ & $>350$ & \\
\hline \multirow[t]{3}{*}{ Apixaban } & $2.5 \mathrm{mg}$ bid & $11-90$ & $30-153$ & $>162$ & $>221$ & \multirow{3}{*}{$\begin{array}{l}25-29 \% \text { renal } \\
56 \% \text { faecal }\end{array}$} \\
\hline & $5 \mathrm{mg}$ bid & $22-177$ & $59-302$ & $>230$ & $>321$ & \\
\hline & 10 mg bid & $41-335$ & $111-572$ & $>335$ & $>572$ & \\
\hline \multirow[t]{2}{*}{ Edoxaban } & $60 \mathrm{mg}$ od & $10-40$ & $120-150$ & $?$ & $?$ & $35 \%$ renal \\
\hline & $30 \mathrm{mg}$ od & $?$ & $?$ & ? & $?$ & $65 \%$ faecal \\
\hline
\end{tabular}


These cases illustrate that on an individual basis there may be several reasons to consider quantitative analysis of DOAC levels to improve clinical decision making. However, there are still important difficulties in the interpretation of DOAC levels:

1. The correlation between the different doses of DOACs and measured drug levels is rather weak, reflected by broad "on-therapy" ranges. ${ }^{50,52-54}$ Although for some DOACs drug levels are associated with clinical outcome, such as bleeding and thrombosis ${ }^{45-47}$, this has not been translated into a clinically useful therapeutic window.

2. Studying DOAC levels and outcome is complicated by the short half-life time. Knowledge of type of DOAC, dose and the exact time of last drug intake is crucial for the interpretation of DOAC levels.

3. For each DOAC a reduced dose regimen, selected for patient with certain characteristics (Table 1), has been established. However, the recommendations for switching to a lower dose differ per DOAC. This way, a patient could, with the same characteristics, qualify for a lower dose of one DOAC but for a full dose of the other. Moreover, patient characteristics can change over time e.g. temporarily impaired renal or liver function. The guidelines advise monitoring of kidney function during DOAC treatment, but the frequency by which this needs to be done is unknown. Besides, a recent real life study did not find a correlation between DOAC levels and kidney function. ${ }^{55}$

4. It remains uncertain whether DOAC levels represent the full effect of DOACs on global coagulation (activity), or even relate to clinical outcome (thrombotic or bleeding events), although recent studies suggest linkage of trough levels to thromboembolic events. ${ }^{47}$ 
Table 3: Summary of possible tests to assess DOACs, adapted from Douxfils et al..$^{44}$

\begin{tabular}{|c|c|c|c|c|}
\hline Lab test & Interpretation: & To keep in mind: & Type of Test & Suitable to asses: \\
\hline aPTT & $\begin{array}{l}\text { Normal aPTT: Excludes } \\
\text { above "on-therapy" levels of } \\
\text { dabigatran } \\
\text { Does NOT exclude the presence } \\
\text { of dabigatran }\end{array}$ & $\begin{array}{l}\text { Sensitivity is } \\
\text { highly dependent } \\
\text { on reagent. }\end{array}$ & Clotting time & Dabigatran \\
\hline $\mathrm{TT}$ & $\begin{array}{l}\text { Normal TT: Excludes the } \\
\text { presence of dabigatran } \\
\text { Does NOT tell you how much } \\
\text { dabigatran is present }\end{array}$ & $\begin{array}{l}\text { Can be prolonged } \\
\text { even in the } \\
\text { presence of } \\
\text { non-relevant drug } \\
\text { levels. }\end{array}$ & Clotting time & Dabigatran \\
\hline PT & $\begin{array}{l}\text { Normal PT: Excludes above "on- } \\
\text { therapy" levels of rivaroxaban } \\
\text { (or edoxaban at peak). } \\
\text { Does NOT exclude the presence } \\
\text { of rivaroxaban or edoxaban at } \\
\text { trough levels. }\end{array}$ & $\begin{array}{l}\text { Sensitivity is } \\
\text { highly dependent } \\
\text { on reagent. }\end{array}$ & Clotting time & $\begin{array}{l}\text { Rivaroxban } \\
\text { (edoxaban) }\end{array}$ \\
\hline $\begin{array}{l}\text { dTT } \\
\text { ECA/ECT }\end{array}$ & $\begin{array}{l}\text { Sensitive for the detection of drug } \\
\text { levels of dabigatran. } \\
\text { Good correlation with LC-MS/MS. }\end{array}$ & $\begin{array}{l}\text { Not available in } \\
\text { every hospital. } \\
\text { Requires specific } \\
\text { calibrator. }\end{array}$ & $\begin{array}{l}\text { Quantification of } \\
\text { DTI levels }\end{array}$ & Dabigatran \\
\hline $\begin{array}{l}\text { Chromogenic } \\
\text { anti-Xa assay }\end{array}$ & $\begin{array}{l}\text { Sensitive in detecting drug levels } \\
\text { of rivaroxaban, apixaban and } \\
\text { edoxaban. } \\
\text { Good correlation with LC-MS/MS. }\end{array}$ & $\begin{array}{l}\text { Not available in } \\
\text { every hospital. } \\
\text { Requires specific } \\
\text { calibrator. }\end{array}$ & $\begin{array}{l}\text { Quantification } \\
\text { direct FXa levels }\end{array}$ & $\begin{array}{l}\text { Rivaroxban } \\
\text { Apixaban } \\
\text { Edoxaban }\end{array}$ \\
\hline dRVV-T & $\begin{array}{l}\text { Normal dRVV-T: can exclude } \\
\text { DOAC levels }>50 \mathrm{ng} / \mathrm{mL}^{-1} \\
\text { Does not tell you how much } \\
\text { DOAC is present }\end{array}$ & $\begin{array}{l}\text { Sensitivity is } \\
\text { dependent on the } \\
\text { reagent but }<\text { than } \\
\text { PT and aPTT. }\end{array}$ & Clotting time & $\begin{array}{l}\text { Can be used for } \\
\text { all DOACs }\end{array}$ \\
\hline LC-MS/MS & $\begin{array}{l}\text { Most reliable tool to determine } \\
\text { drug levels. } \\
\text { Only technique that can } \\
\text { discriminate between DOACs. }\end{array}$ & $\begin{array}{l}\text { Only available in } \\
\text { specialized labs. } \\
\text { Requires trained } \\
\text { staff. } \\
\text { Time consuming. }\end{array}$ & $\begin{array}{l}\text { Quantification of } \\
\text { drug levels }\end{array}$ & $\begin{array}{l}\text { Can be used for } \\
\text { all DOACs }\end{array}$ \\
\hline
\end{tabular}

activated partial thromboplastin time (aPTT), thrombin time (TT), prothrombin time (PT), diluted thrombin time (dTT), ecarin chromogenic assay (ECA) ecarin clotting time (ECT); direct thrombin inhibitors (DTI), diluted Russell's viper venom time (dRVV-T), direct oral anticoagulant (DOAC), liquid chromatography tandem mass spectrometry (LC-MS/MS) 


\section{MEASURING DOACS IN A FUNCTIONAL MANNER}

Measuring DOACs by most conventional clotting time-based assays is hindered by a lack of the required sensitivity and a lack of correlation between a prolonged clotting time and anticoagulant intensity. ${ }^{42,52,56-60}$ In table 3 an overview is given of several quantitative assays and their applicability to measure DOACs. The accurate quantification of DOAC levels in patients can be performed with sensitive assays that are currently present in most hospitals in the Netherlands. ${ }^{44,60-63}$ However, DOAC levels may not accurately represent the effect of DOACs on coagulation in individual patients. A global coagulation assay, such as the thrombin generation (TG) assay, is able to detect DOACs and can display their effect on coagulation. ${ }^{61,64,65} \mathrm{TG}$ can be measured automatically by several methods. One of the best-known methods is calibrated automated thrombinography (CAT). This assay, which estimates the amount of free thrombin formed over time, was developed by Hemker et al. ${ }^{66}$ Since its development, TG measured by CAT, has shown correlations with clinical outcomes and is being used in many laboratories around the world. ${ }^{67-70}$ Depending on the initiator used, different parts of the coagulation system can be studied, e.g. at low TF concentrations the Josso loop plays a more pronounced role, and in the presence of TM the APC system can be assessed. In addition to testing global coagulation profiles, TG has the capacity to detect the effect of many anticoagulants. ${ }^{71,72}$ However, due to the large array of possibilities there are difficulties in standardization and therefore the various modifications for DOAC testing have not yet been implemented.

When measuring TG with CAT, coagulation is usually initiated by TF. Lowering the concentration of TF increases the sensitivity of the assay. The reaction is started by the recalcification of citrated plasma. A fluorescent substrate is added with a low affinity for thrombin. The total quantity of free thrombin can be calculated by comparing the intensity of measured fluorescence, to the fluorescence intensity of a matched calibrator $\left(a_{2}\right.$-macroglobulin $\left(a_{2} M\right)$ thrombin) with a known amount of thrombin activity. The generated thrombin can bind to AT, which will lead to direct inactivation of thrombin. However, a part of thrombin (estimated at $25 \%$ ) can bind to $\mathrm{a}_{2} \mathrm{M}$ forming the complex $\mathrm{a}_{2} \mathrm{M}$-thrombin, which can still cleave small substrates (such as the fluorescent substrate used in CAT). Therefore, after the experiment, a correction is made by subtracting $\mathrm{a}_{2} \mathrm{M}$-thrombin from the total amount of thrombin measured. The outcome of TG is often presented by several numerical parameters, but can also be graphically displayed as a thrombogram, shown in figure 6 . The thrombogram 
starts with the initiation phase, called the lag time. The lag time presents the time from the addition of a trigger to the moment the first thrombin is formed, and can be compared with a clotting time. The highest point of the graph, represents the maximum amount of thrombin and is known as the peak. The time-to-peak (TTP) is the time needed to reach the peak. The total amount of thrombin that can be formed over time is represented by the area under the curve, named the endogenous thrombin potential (ETP). The steepness of the rising curve, or velocity index, characterizes the speed of thrombin formation. The downward slope of the curve characterizes the speed of its decay $\left(\mathrm{K}_{\text {decay }}\right)$, which is mainly the effect of AT that inactivates $2 / 3$ of thrombin. One fourth is absorbed $a_{2} M$, the rest of the thrombin is caught by less important inhibitors.

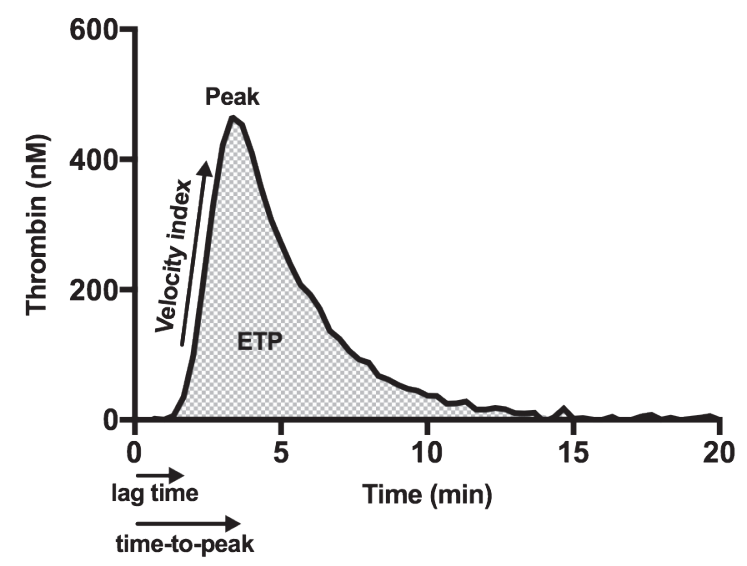

Figure 6. An example of a thrombogram with its mostly used parameters.

ETP, endogenous thrombin potential

As mentioned above, TG is able to distinguish prothrombotic as well as bleeding phenotypes in patients. 67,70,73,74 In addition to testing global coagulation profiles, TG has the capacity to detect the effect of many anticoagulants ${ }^{71,72}$. Multiple studies have shown that TG is also able to detect the presence of DOACs, and might be able to display the impact of DOAC on global coagulation. In figure 7 the dose dependent effect of rivaroxaban, a direct FXa inhibitor, on the TG curve is shown. If the inhibition of these curves could be correlated to clinical outcome would that make TG a suitable tool to assess the impact of DOACs? Would the results of TG be a valuable addition to measuring DOAC levels? In this thesis I will hope to answers to some of these questions. 


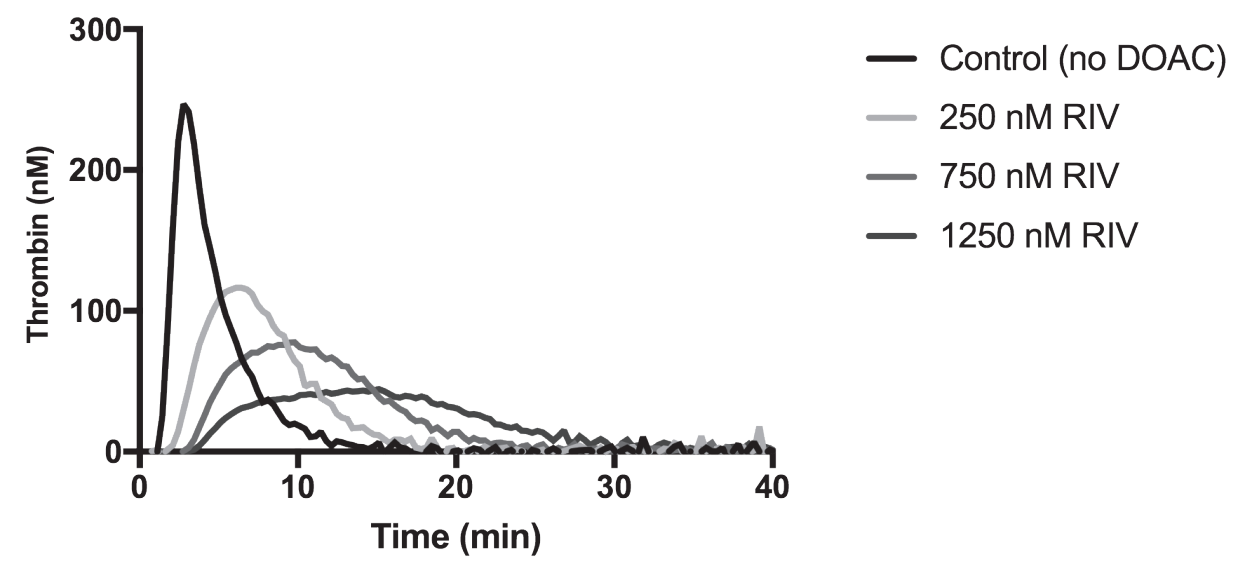

Figure 7. An example of the dose-dependent response of rivaroxaban (RIV) on thrombin generation.

\section{OUTLINE OF THIS THESIS}

In this thesis several aspects of measuring anticoagulation therapy with TG are described. In chapter 2 different tools that could predict of bleeding in patients using VKAs are investigated, for instance the HAS-BLAD score and TG, in whole blood as well as in plasma. In chapter $\mathbf{3}$ an overview is provided of the current literature on the ability of TG to predict bleeding in patients using VKAs. In chapter $\mathbf{4}$ the ability of TG to measure the direct thrombin inhibitor dabigatran is assessed. Chapter $\mathbf{5}$ is about the development of an assay to measure levels of direct FXa inhibitors concomitant with TG in the same set-up. In chapter 6 the in vitro inter- and intra-individual variation is examined in TG upon addition of fixed dose of DOACs and over a longer period of time. Chapter 7 describes the differential effects of direct thrombin inhibitors and direct FXa inhibitors on fibrinolysis, using a turbidimetric assay. The focus is especially on the TAFI-pathway. In chapter $\mathbf{8}$ all the results are summarized and discussed. 


\section{REFERENCES}

1. Dahlback B. Blood coagulation. Lancet. 2000;355(9215):1627-1632

2. Furie B, Furie BC. Mechanisms of thrombus formation. N Engl J Med. 2008;359(9):938-949

3. Versteeg HH, Heemskerk JW, Levi M, Reitsma PH. New fundamentals in hemostasis. Physiol Rev. 2013;93(1):327-358.

4. Bouma BN, Meijers JC. Role of blood coagulation factor XI in downregulation of fibrinolysis. Curr Opin Hematol. 2000;7(5):266-272.

5. Rosing J, Tans G, Govers-Riemslag JW, Zwaal RF, Hemker HC. The role of phospholipids and factor Va in the prothrombinase complex. J Biol Chem. 1980;255(1):274-283.

6. Nesheim ME, Taswell JB, Mann KG. The contribution of bovine Factor $V$ and Factor Va to the activity of prothrombinase. J Biol Chem. 1979;254(21):10952-10962.

7. Xi M, Beguin S, Hemker HC. Importance of factor-IX-dependent prothrombinase formation--the Josso pathway--in clotting plasma. Haemostasis. 1989;19(6):301-308.

8. Mann KG, Brummel K, Butenas S. What is all that thrombin for? J Thromb Haemost. 2003;1(7):1504-1514.

9. Josso F, Prou-Wartelle O. Interaction of tissue factor and factor VII at the earliest phase of coagulation. Thromb Diath Haemorrh Suppl. 1965;17:35-44.

10. Lorand L. Factor XIII: structure, activation, and interactions with fibrinogen and fibrin. Ann N Y Acad Sci. 2001;936:291-311.

11. Rosing J, Hoekema L, Nicolaes GA, et al. Effects of protein S and factor Xa on peptide bond cleavages during inactivation of factor Va and factor VaR506Q by activated protein C. J Biol Chem. 1995;270(46):27852-27858.

12. Shen $L$, Dahlback B. Factor $V$ and protein $S$ as synergistic cofactors to activated protein $C$ in degradation of factor VIIla. J Biol Chem. 1994;269(29):18735-18738.

13. Hackeng TM, Rosing J. Protein S as cofactor for TFPI. Arterioscler Thromb Vasc Biol. 2009;29(12):2015-2020.

14. Chapin JC, Hajjar KA. Fibrinolysis and the control of blood coagulation. Blood Rev. 2015;29(1):17-24.

15. Wolberg AS. Thrombin generation and fibrin clot structure. Blood Rev. 2007;21(3):131-142.

16. Bajzar $\mathrm{L}$. Thrombin activatable fibrinolysis inhibitor and an antifibrinolytic pathway. Arterioscler Thromb Vasc Biol. 2000;20(12):2511-2518.

17. Mosnier LO, Bouma BN. Regulation of fibrinolysis by thrombin activatable fibrinolysis inhibitor, an unstable carboxypeptidase B that unites the pathways of coagulation and fibrinolysis. Arterioscler Thromb Vasc Biol. 2006;26(11):2445-2453

18. Lisman T, Leebeek FW, Mosnier LO, et al. Thrombin-activatable fibrinolysis inhibitor deficiency in cirrhosis is not associated with increased plasma fibrinolysis. Gastroenterology. 2001;121(1):131-139.

19. Bagot CN, Arya R. Virchow and his triad: a question of attribution. Br J Haematol. 2008;143(2):180-190.

20. Mieres $\mathrm{JH}$. Review of the American Heart Association's guidelines for cardiovascular disease prevention in women. Heart. 2006;92 Suppl 3:iii10-13

21. Townsend N, Wilson L, Bhatnagar P, Wickramasinghe K, Rayner M, Nichols M. Cardiovascular disease in Europe: epidemiological update 2016. Eur Heart J. 2016;37(42):3232-3245.

22. Zoni-Berisso M, Lercari F, Carazza T, Domenicucci S. Epidemiology of atrial fibrillation: European perspective. Clin Epidemiol. 2014;6:213-220.

23. Lip GY, Nieuwlaat R, Pisters R, Lane DA, Crijns HJ. Refining clinical risk stratification for predicting stroke and thromboembolism in atrial fibrillation using a novel risk factor-based approach: the euro heart survey on atrial fibrillation. Chest. 2010;137(2):263-272.

24. Choi HI, Ahn JM, Kang SH, et al. Prevalence, Management, and Long-Term (6-Year) Outcomes of Atrial Fibrillation Among Patients Receiving Drug-Eluting Coronary Stents. JACC Cardiovasc Interv. 2017;10(11):1075-1085. 
25. Enomoto $Y$, lijima $R$, Tokue $M$, et al. Bleeding risk with triple antithrombotic therapy in patients with atrial fibrillation and drug-eluting stents. Cardiovasc Interv Ther. 2014;29(3):193-199.

26. Kang DO, Yu CW, Kim HD, et al. Triple antithrombotic therapy versus dual antiplatelet therapy in patients with atrial fibrillation undergoing drug-eluting stent implantation. Coron Artery Dis. 2015;26(5):372-380.

27. Shehab N, Lovegrove MC, Geller Al, Rose KO, Weidle NJ, Budnitz DS. US Emergency Department Visits for Outpatient Adverse Drug Events, 2013-2014. Jama. 2016;316(20):2115-2125.

28. Piazza G, Nguyen TN, Cios D, et al. Anticoagulation-associated adverse drug events. Am J Med. 2011;124(12):1136-1142.

29. Blann AD, Fitzmaurice DA, Lip GY. Anticoagulation in hospitals and general practice. Bmj. 2003;326(7381):153-156.

30. Rubboli A, Becattini C, Verheugt FW. Incidence, clinical impact and risk of bleeding during oral anticoagulation therapy. World J Cardiol. 2011;3(11):351-358.

31. Connolly SJ, Ezekowitz MD, Yusuf S, et al. Dabigatran versus warfarin in patients with atrial fibrillation. N Engl J Med. 2009;361(12):1139-1151.

32. Patel MR, Mahaffey KW, Garg J, et al. Rivaroxaban versus warfarin in nonvalvular atrial fibrillation. N Engl J Med. 2011;365(10):883-891.

33. Granger $\mathrm{CB}$, Alexander JH, McMurray JJ, et al. Apixaban versus warfarin in patients with atrial fibrillation. N Engl J Med. 2011;365(11):981-992.

34. Giugliano RP, Ruff CT, Braunwald E, et al. Edoxaban versus warfarin in patients with atrial fibrillation. N Engl $J$ Med. 2013;369(22):2093-2104.

35. Bauersachs R, Berkowitz SD, Brenner B, et al. Oral rivaroxaban for symptomatic venous thromboembolism. N Engl J Med. 2010;363(26):2499-2510.

36. Schulman S, Kearon C, Kakkar AK, et al. Dabigatran versus warfarin in the treatment of acute venous thromboembolism. N Engl J Med. 2009;361(24):2342-2352.

37. Agnelli G, Buller HR, Cohen A, et al. Oral apixaban for the treatment of acute venous thromboembolism. N Engl J Med. 2013;369(9):799-808.

38. Buller HR, Decousus H, Grosso MA, et al. Edoxaban versus warfarin for the treatment of symptomatic venous thromboembolism. N Engl J Med. 2013;369(15):1406-1415.

39. Eikelboom JW, Connolly SJ, Bosch J, et al. Rivaroxaban with or without Aspirin in Stable Cardiovascular Disease. N Engl J Med. 2017;377(14):1319-1330.

40. Heidbuchel H, Verhamme P, Alings M, et al. Updated European Heart Rhythm Association Practical Guide on the use of non-vitamin $\mathrm{K}$ antagonist anticoagulants in patients with non-valvular atrial fibrillation. Europace. 2015;17(10):1467-1507.

41. Levy JH, Ageno W, Chan NC, Crowther M, Verhamme P, Weitz JI. When and how to use antidotes for the reversal of direct oral anticoagulants: guidance from the SSC of the ISTH. J Thromb Haemost. 2016;14(3):623-627.

42. Baglin T, Hillarp A, Tripodi A, Elalamy I, Buller H, Ageno W. Measuring Oral Direct Inhibitors (ODIs) of thrombin and factor Xa: A recommendation from the Subcommittee on Control of Anticoagulation of the Scientific and Standardisation Committee of the International Society on Thrombosis and Haemostasis. J Thromb Haemost. 2013.

43. Dubois $\mathrm{V}$, Dincq AS, Douxfils $\mathrm{J}$, et al. Perioperative management of patients on direct oral anticoagulants. Thromb J. 2017;15:14.

44. Douxfils J, Ageno W, Samama CM, et al. Laboratory testing in patients treated with direct oral anticoagulants: a practical guide for clinicians. J Thromb Haemost. 2018;16(2):209-219.

45. Reilly PA, Lehr T, Haertter S, et al. The effect of dabigatran plasma concentrations and patient characteristics on the frequency of ischemic stroke and major bleeding in atrial fibrillation patients: the RE-LY Trial (Randomized Evaluation of Long-Term Anticoagulation Therapy). J Am Coll Cardiol. 2014;63(4):321-328. 
46. Aisenberg J, Chatterjee-Murphy P, Friedman Flack K, et al. Gastrointestinal Bleeding With Edoxaban Versus Warfarin: Results From the ENGAGE AF-TIMI 48 Trial (Effective Anticoagulation With Factor Xa Next Generation in Atrial Fibrillation-Thrombolysis In Myocardial Infarction). Circ Cardiovasc Qual Outcomes. 2018;11(5):e003998.

47. Testa S, Paoletti O, Legnani C, et al. Low drug levels and thrombotic complications in high-risk atrial fibrillation patients treated with direct oral anticoagulants. J Thromb Haemost. 2018;16(5):842-848.

48. Gibson $\mathrm{CM}$, Mehran R, Bode C, et al. Prevention of Bleeding in Patients with Atrial Fibrillation Undergoing PCI. N Engl J Med. 2016;375(25):2423-2434.

49. Dincq AS, Lessire S, Douxfils J, Dogne JM, Gourdin M, Mullier F. Management of non-vitamin K antagonist oral anticoagulants in the perioperative setting. Biomed Res Int. 2014;2014:385014.

50. Francart SJ, Hawes EM, Deal AM, et al. Performance of coagulation tests in patients on therapeutic doses of rivaroxaban. A cross-sectional pharmacodynamic study based on peak and trough plasma levels. Thromb Haemost. 2014;111(6):1133-1140.

51. Skeppholm M, Hjemdahl P, Antovic JP, et al. On the monitoring of dabigatran treatment in "real life" patients with atrial fibrillation. Thromb Res. 2014;134(4):783-789.

52. Cuker A, Siegal DM, Crowther MA, Garcia DA. Laboratory measurement of the anticoagulant activity of the non-vitamin K oral anticoagulants. J Am Coll Cardiol. 2014;64(11):1128-1139.

53. Hawes EM, Deal AM, Funk-Adcock D, et al. Performance of coagulation tests in patients on therapeutic doses of dabigatran: a cross-sectional pharmacodynamic study based on peak and trough plasma levels. J Thromb Haemost. 2013;11(8):1493-1502.

54. Samuelson BT, Cuker A. Measurement and reversal of the direct oral anticoagulants. Blood Rev. 2017;31(1):77-84

55. Testa S, Tripodi A, Legnani $C$, et al. Plasma levels of direct oral anticoagulants in real life patients with atrial fibrillation: Results observed in four anticoagulation clinics. Thromb Res. 2016;137:178-183.

56. Kitchen S, Gray E, Mackie I, Baglin T, Makris M, committee B. Measurement of non-coumarin anticoagulants and their effects on tests of Haemostasis: Guidance from the British Committee for Standards in Haematology. Br J Haematol. 2014;166(6):830-841.

57. Samuelson BT, Cuker A. Measurement and reversal of the direct oral anticoagulants. Blood Rev. 2016.

58. Tripodi A. To measure or not to measure direct oral anticoagulants before surgery or invasive procedures. $J$ Thromb Haemost. 2016;14(7):1325-1327.

59. Avecilla ST, Ferrell C, Chandler WL, Reyes M. Plasma-diluted thrombin time to measure dabigatran concentrations during dabigatran etexilate therapy. Am J Clin Pathol. 2012;137(4):572-574.

60. Douxfils J, Chatelain B, Hjemdahl P, et al. Does the Russell Viper Venom time test provide a rapid estimation of the intensity of oral anticoagulation? A cohort study. Thromb Res. 2015;135(5):852-860.

61. Douxfils J, Chatelain C, Chatelain B, Dogne JM, Mullier F. Impact of apixaban on routine and specific coagulation assays: a practical laboratory guide. Thromb Haemost. 2013;110(2):283-294.

62. Douxfils J, Dogne JM, Mullier F, et al. Comparison of calibrated dilute thrombin time and aPTT tests with LC-MS/MS for the therapeutic monitoring of patients treated with dabigatran etexilate. Thromb Haemost. 2013;110(3):543-549.

63. Douxfils J, Tamigniau A, Chatelain B, et al. Comparison of calibrated chromogenic anti-Xa assay and PT tests with LC-MS/MS for the therapeutic monitoring of patients treated with rivaroxaban. Thromb Haemost. 2013;110(4):723-731.

64. Douxfils J, Mullier F, Robert S, Chatelain C, Chatelain B, Dogne JM. Impact of dabigatran on a large panel of routine or specific coagulation assays. Laboratory recommendations for monitoring of dabigatran etexilate. Thromb Haemost. 2012;107(5):985-997.

65. Douxfils J, Mullier F, Loosen C, Chatelain C, Chatelain B, Dogne JM. Assessment of the impact of rivaroxaban on coagulation assays: laboratory recommendations for the monitoring of rivaroxaban and review of the literature. Thromb Res. 2012;130(6):956-966. 
66. Hemker HC, Giesen P, Al Dieri R, et al. Calibrated automated thrombin generation measurement in clotting plasma. Pathophysiol Haemost Thromb. 2003;33(1):4-15.

67. Bloemen S, Zwaveling S, Ten Cate H, Ten Cate-Hoek A, de Laat B. Prediction of bleeding risk in patients taking vitamin K antagonists using thrombin generation testing. PLoS One. 2017;12(5):e0176967.

68. Hron G, Kollars M, Binder BR, Eichinger S, Kyrle PA. Identification of patients at low risk for recurrent venous thromboembolism by measuring thrombin generation. Jama. 2006;296(4):397-402.

69. Segers $\mathrm{O}$, van Oerle R, ten Cate H, Rosing J, Castoldi E. Thrombin generation as an intermediate phenotype for venous thrombosis. Thromb Haemost. 2010;103(1):114-122.

70. Tripodi A, Legnani C, Chantarangkul V, Cosmi B, Palareti G, Mannucci PM. High thrombin generation measured in the presence of thrombomodulin is associated with an increased risk of recurrent venous thromboembolism. J Thromb Haemost. 2008;6(8):1327-1333.

71. Bloemen S, Hemker HC, Al Dieri R. Large inter-individual variation of the pharmacodynamic effect of anticoagulant drugs on thrombin generation. Haematologica. 2013;98(4):549-554.

72. Gatt A, van Veen JJ, Woolley AM, Kitchen S, Cooper P, Makris M. Thrombin generation assays are superior to traditional tests in assessing anticoagulation reversal in vitro. Thromb Haemost. 2008;100(2):350-355.

73. Al Dieri R, Peyvandi F, Santagostino E, et al. The thrombogram in rare inherited coagulation disorders: its relation to clinical bleeding. Thromb Haemost. 2002;88(4):576-582.

74. Hemker HC, Giesen P, AIDieri R, et al. The calibrated automated thrombogram (CAT): a universal routine test for hyper- and hypocoagulability. Pathophysiol Haemost Thromb. 2002;32(5-6):249-253. 


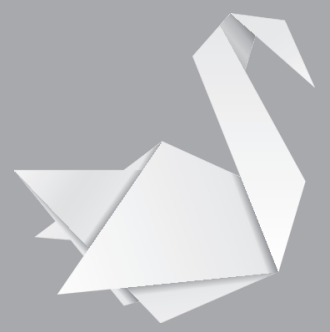




\section{CHAPTER}

Prediction of bleeding risk in patients taking vitamin $\mathrm{K}$ antagonists using thrombin generation testing

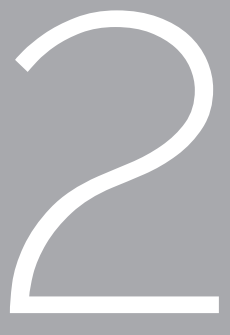

Saartje Bloemen*

Suzanne Zwaveling*

Hugo ten Cate

Arina ten Cate-Hoek

Bas de Laat

*These authors contributed equally to this work. 


\section{ABSTRACT}

\section{Background}

Until recently, vitamin $\mathrm{K}$ antagonists (VKAs) were the mainstay of oral anticoagulant treatment with bleeding as the most prevalent adverse effect. One to four percent of patients experience major bleeding episodes, while clinically relevant bleeding occurs in up to $20 \%$. At this moment no laboratory assays are available to identify patients at risk for bleeding. With this study we aimed to investigate whether thrombin generation tests might identify a bleeding risk in patients taking VKAs.

\section{Methods}

This prospective cohort study included 129 patients taking VKAs for more than three months. Calibrated automated thrombinography (CAT) was performed in whole blood, platelet rich and platelet poor plasma. Hematocrit, hemoglobin concentrations and the International Normalized Ratio (INR) were defined and coagulation factor levels were measured.

\section{Results}

Forty clinically relevant bleeding episodes were registered in 26 patients during follow-up. No differences were found in plasma CAT parameters or INR values. Bleeding was not associated with age, sex, hematocrit, hemoglobin levels or coagulation factor levels. In whole blood a significantly lower endogenous thrombin potential (ETP) and peak were found in patients with bleeding (median ETP: 182.5 versus $256.2 \mathrm{nM}$.min, $\mathrm{p}=0.002$; peak: 23.9 versus $39.1 \mathrm{nM}, \mathrm{p}=0.029)$. Additionally, the area under the receiver operating curve (AUC ROC) was significantly associated with bleeding (ETP: 0.700, $p=0.002$; peak: 0.642, $p=0.029$ ). HAS-BLED scores were also significantly higher in bleeding patients ( 3 versus $2, p=0.003$ ), with an AUC ROC $0.682(p=0.004)$.

\section{Conclusions}

In conclusion, bleeding in patients taking VKAs is associated with a decreased whole blood ETP and peak as well as with an increased HAS-BLED score. 


\section{INTRODUCTION}

For over 50 years vitamin $\mathrm{K}$ antagonists (VKAs) have been widely used, not only as (first choice) treatment for thromboembolism, but as primary and secondary prevention of (venous) thromboembolism as well. ' Warfarin is currently the most prescribed VKAs followed by acenocoumarol and phenprocoumon. The predominant adverse effect of anticoagulant therapy is an increased risk of bleeding which can lead to morbidity and mortality. Annually approximately 1 to $4 \%$ of patients treated with VKAs suffer from major bleeding episode. ${ }^{2}$ Clinically relevant bleeding occurs in up to $20 \%$ of patients. ${ }^{3}$ The risk of bleeding increases with age. Patients that are older than 75 years, experience major bleeding more frequently than younger patients: $5.1 \%$ versus $1 \%$ per year, respectively. ${ }^{4}$ This bleeding risk increases even more when VKAs are combined with antiplatelet therapy. ${ }^{5}$ In the past several attempts were made to more accurately estimate the bleeding risk of individual patients treated with VKAs. One of the commonly used clinical methods for the identification of patients with atrial fibrillation at risk for bleeding is the HAS-BLED score, which is a clinical decision score. ${ }^{6}$ The HAS-BLED score contains the risk factors hypertension, abnormal renal/liver function, stroke, bleeding history or predisposition, labile international normalized ratio (INR), elderly (age $\geq 65$ years) and drugs/alcohol (ab)use concomitantly. Although the HAS-BLED score is developed and validated only in patients with $A F$, it would be reasonable to think that the score could be applied in patients with different indications for VKA use, considering the comparable risk factors for bleeding. Moreover, the HAS-BLED score has the highest predictive potential compared to other clinical prediction scores $^{7}$; however its accuracy differed based on the cohort used for validation. 8 ,9 As of yet there are no laboratory methods that prospectively predict which patients are at risk for bleeding. Considering the INR, there is an increased risk of bleeding at higher INR levels, yet the majority of bleeding events occurs in patients that are within the therapeutic range. Thrombin generation, a method that detects the enzymatic activity of thrombin, has been shown to be able to detect both prothrombotic and bleeding phenotypes based on changes in the coagulation system. ${ }^{10}$ Additionally, thrombin generation has the capacity to detect the anticoagulant effect of many if not all anticoagulants, including VKAs and direct oral anticoagulants (DOACs). 11,12 Until recently this method was only applicable in plasma due to quenching of the fluorescent signal by sedimentation of erythrocytes. Introduction of a porous matrix, preventing this sedimentation, and using a different thrombin-sensitive substrate enabled studying thrombin generation in whole blood. ${ }^{13}$ In this study we investigated whether thrombin generation, in plasma or whole blood, could be used to predict bleeding episodes in 129 patients taking VKAs and compared these parameters to the INR, the HAS-BLED score, fibrinogen levels and other factor determinations. 


\section{MATERIALS \& METHODS}

\section{Study population}

Patients taking VKAs were randomly included in this study between March 2012 and October 2013. A sample size of 127 was sufficient to provide power of $80 \%$ with a two-sided a-level of 0.05. Patients were eligible for inclusion in this study when treated with VKAs for longer than three months and undergoing a venapuncture in order to determine their INR value at the Maastricht anticoagulation clinic. Patients under 18 years of age were excluded. All patients were informed and provided written consent. The study was approved by the local medical ethical committee (Medisch-ethische toetsingscommissie academisch ziekenhuis Maastricht/universiteit Maastricht (METC aZM/UM), approval number: 11-4-142.4/ccl).

\section{Blood samples}

Patients were included in the anticoagulation clinic during one of their routine checkups for VKA treatment. After giving informed consent on this day of inclusion, blood was drawn for both INR determination and further laboratory assays necessary for this study, such as thrombin generation. Blood was collected through antecubital venapuncture using citrate tubes (1 volume of trisodium citrate $3.2 \%$ to 9 volumes of blood), (BD Vacutainer system, Roborough, Plymouth, UK). Platelet rich plasma (PRP) was prepared by centrifuging the blood at $250 \mathrm{~g}$ for $15 \mathrm{~min}$. Platelet poor plasma (PPP) was prepared by double centrifugation at 2,000 g for $10 \mathrm{~min}$. PPP for thrombin generation was used immediately, the remainder was stored at $-80^{\circ} \mathrm{C}$ until bulk analysis in other assays was possible.

\section{Follow-up}

Bleeding episodes were recorded during the follow-up period (minimum four months, mean follow-up time: 15.5 months) at the anticoagulation clinic in Maastricht according to the definitions and criteria defined by the Dutch Federation of Anticoagulation Clinics. These criteria are based on the guidelines by the Scientific and Standardization Committee of the International Society on Thrombosis and Haemostasis. ${ }^{14}$ The definitions of major bleeding are: any intracranial hemorrhage, any objectively diagnosed intra-articular hemorrhage and bleeding leading to death, transfusion, surgery, and/or hospital admission. Minor bleeding was defined as all other clinically relevant bleeding not meeting the definition for major bleeding. Clinically relevant bleeding was defined according to the guidelines of the Dutch Federation of Anticoagulation Clinics. Bleeding events, when detected by a general 
practitioner or at the hospital, were systematically reported to the anticoagulation clinic. Minor bleedings were mostly reported by patients themselves, during routine visits and only rated if they were clinically relevant. The specifications of the types of bleeding (minor/ major) in our study are listed in Table 1.

Table 1. Bleeding types with specifications and the number of each type of bleeding that occurred during the follow-up period.

\begin{tabular}{llll} 
Type & Specification & Classification & Number of bleedings \\
\hline Nose & $>30 \mathrm{~min}$ & Minor & 6 \\
Eye & conjunctival & Minor & 12 \\
\hline Skin & $>10 \mathrm{~cm}$ or multiple hematomas & Minor & 7 \\
\hline Digestive tract & & Minor & 3 \\
\hline Uro-genital tract & & Minor & 5 \\
\hline Traumatic bleed & 30 min, with treatment & Minor & 2 \\
\hline Nose & & Major & 2 \\
\hline Digestive tract & Major & 2 \\
\hline Other locations & & Minor & 1
\end{tabular}

We also recorded age, sex, type of VKA, time in therapeutic range (TTR) and indication for VKA use. The use of antithrombotic therapy as well as other co-medications affecting coagulation (e.g. non-steroidal anti-inflammatory drugs (NSAIDs)) was also documented to assess potential confounding effects. Bleeding episodes were recorded until the end of the study or until discontinuation of treatment. Other parameters were determined on the day of inclusion.

\section{Reagents}

Synthetic phospholipids were obtained from Avanti Polar Lipids Inc. (Alabaster, AL, USA). Recombinant tissue factor (TF) known as Innovin (Dade-Behring, Marburg, Germany) was used. Z-Gly-Gly-Arg-aminomethylcoumarine (ZGGR-AMC) was purchased from Bachem (Basel, Switzerland). Rhodamine substrate ( $\mathrm{P}_{2} \mathrm{Rho}$ ) was a gift of Diagnostica Stago (Asnières sur Seine, France). Recombinant human thrombomodulin (TM) was a kind gift of Asahi Kasei Pharma (Japan). The calibrator, a2-macroglobulin-thrombin complex, was prepared as described previously. ${ }^{15}$ Hepes buffers containing $5 \mathrm{mg} / \mathrm{ml}$ or $60 \mathrm{mg} / \mathrm{ml}$ bovine serum albumin were prepared as described by Hemker et al. ${ }^{16}$ 


\section{Thrombin generation}

CAT was performed in plasma as described earlier. ${ }^{15}$ TF was used to initiate the reaction at a final concentration of $1 \mathrm{pM}$ in PRP and at both $1 \mathrm{pM}$ and $5 \mathrm{pM}$ with $4 \mu \mathrm{M}$ phospholipids in PPP. The effect of TM was also tested in PPP (1 pM TF) at a concentration of $2.5 \mathrm{nM}$. In PPP (5 pM TF) and PRP $20 \mathrm{nM}$ of TM was used (around IC ${ }_{50}$ ). The whole blood CAT technique was performed according to our group's earlier specifications. ${ }^{13}$ Thirty microliters of blood was mixed with $10 \mu \mathrm{l}$ of P2Rho substrate $(1.8 \mathrm{mM})$ and $20 \mu \mathrm{l}$ of $\mathrm{TF} / \mathrm{CaCl}_{2}$ solution was added, which initiated thrombin generation. In the calibration wells, $20 \mu$ l of reagents were replaced with calibrator (final concentration: $100 \mathrm{nM}$ ). Instantly after the activation, $5 \mu$ l of the mixture is pipetted on paper disks (Whatman 589/1, Whatman $\mathrm{GmbH}$, Dassel, Germany) in a flat bottom 96-well polystyrene plate and covered with $40 \mu \mathrm{l}$ of mineral oil (Affymetrix, USB, Cleveland, Ohio, USA). The final TF concentration was $1 \mathrm{pM}$ and TM (20 nM) was added as well. Fluorescent signals were measured using the Fluoroskan Ascent software (Thermo Labsystems, Helsinki, Finland). Measurements were performed in triplicate and fluorescent signals were transformed into thrombin concentrations as described by Hemker et al. ${ }^{17}$ The thrombin generation parameters which were analyzed were: lag time, which is the time until the first traces of thrombin are formed; ETP, the area under the thrombin generation curve, peak level of thrombin formation and the time-to-peak or the time until the thrombin peak is reached.

\section{Additional analyses}

Hematocrit and hemoglobin concentration determinations were performed in citrated blood with a Coulter Counter analyzer (Beckman Coulter, Woerden, the Netherlands). Levels of clotting factors, including fibrinogen were assessed using the STA-R Evolution analyzer (Stago, Asnières sur Seine, France). Factor II, V, VII, VIII, IX, X levels were determined with clotting assays triggered by either a thromboplastin based reagent (FII, FV, FVII, FX) or a kaolin based reagent (FVIII and FIX). Fibrinogen levels were measured using the Clauss method. Protein $\mathrm{C}$ activity was determined by an aPTT based assay, activated by Agkistrodon c. contortrix venom. Protein $S$ was tested in a clotting assay in which the activity of protein $\mathrm{S}$ as a cofactor of protein $\mathrm{C}$ is measured by its effect on factor $\mathrm{Va}$. Antithrombin (ATIII) was determined by a chromogenic measurement. INRs were determined in the local anticoagulation clinic. The prothrombin time was determined in citrated plasma with an automated coagulation analyzer (Sysmex CA 1500, Siemens Diagnostics, the Netherlands) using Innovin ${ }^{\circ}$ (Dade-Behring) as the thromboplastin reagent. The INR value was expressed 
as the ratio of the subject's PT to a normal (control) sample raised to the power of the International Sensitivity Index (ISI); $\left(\mathrm{PT}_{\text {test }} / \mathrm{PT}_{\text {normal }}\right)^{\mid \mathrm{ISI}}$. HAS-BLED scores of each patient were calculated post-hoc by a blinded physician using medical records, allotting one point for each risk factor. ${ }^{18}$

\section{Statistical analysis}

All analyses were performed using Graphpad Prism version 5.00 (Graphpad Software Inc., La Jolla, CA, USA). Patients with missing data were not excluded from the analysis. Correlation analysis of whole blood versus plasma CAT parameters was performed using Pearson correlation test. Patients were divided into two groups (with bleeding and without bleeding). Differences between groups were analyzed via the Mann Whitney $U$ test and represented by medians with interquartile ranges (IQR), range from minimum value to maximum value and 95\% confidence intervals (CI). Receiver operating curves (ROC) were used to investigate the ability of WB CAT and the HAS-BLED score to discriminate between bleeding and non-bleeding patients. The area under the ROC curve (95\% Cl) quantified the predictive value of parameters. Differences between two groups (other than bleeding versus non-bleeding) were also analyzed using the Mann Whitney $U$ test. A two-sided $p$-value of $\leq$ 0.05 was considered statistically significant.

\section{RESULTS}

\section{Patient characteristics}

One hundred and fifty patients were eligible for the study and 21 patients had to be excluded for several reasons: failed blood collection, not fulfilling the inclusion criterion of using VKAs for at least three months, technical problems during measurements or other reasons. The demographics of the remaining 129 patients are listed in Table 2. The average duration of VKA treatment until the inclusion date was approximately 5 years. Therapeutic ranges consisted of INR's from $2.0-3.5(n=103)$ or $2.5-4.0(n=26)$, depending on the indication. 
Table 2. Patient demographics and clinical characteristics.

\begin{tabular}{|c|c|c|c|c|}
\hline & $\begin{array}{l}\text { Total population } \\
(n=129)\end{array}$ & $\begin{array}{l}\text { Non-bleeding } \\
(n=103)\end{array}$ & $\begin{array}{l}\text { Bleeding } \\
(n=26)\end{array}$ & p-value \\
\hline Age (median [IQR]) & $70[62.5-76.0]$ & $70[60-80]$ & $70[60-80]$ & 0.4074 \\
\hline Female sex $(\%)$ & 22 & 21 & 23 & 0.8532 \\
\hline VKA Acenocoumarol (\%) & 95 & 96.1 & 92.3 & 0.4163 \\
\hline Patients with TTR<60\% (\%) & 80 & 84.5 & 61.5 & 0.0214 \\
\hline \multicolumn{5}{|l|}{ Indications (\%) } \\
\hline AF & 72.1 & 73.8 & 65.4 & \\
\hline prosthetic valve & 13.2 & 10.7 & 23.1 & \\
\hline pulmonary embolism & 3.9 & 3.9 & 3.8 & \\
\hline venous thrombosis & 3.1 & 3.9 & 0 & \\
\hline $\mathrm{CABG}$ & 1.6 & 1.9 & 0 & \\
\hline peripheral atherosclerosis & 1.6 & 1.0 & 3.8 & \\
\hline cardiomyopathy & 0.8 & 1.0 & 0 & \\
\hline cerebrovascular insufficiency & 0.8 & 1.0 & 0 & \\
\hline cerebral embolism & 0.8 & 1.0 & 0 & \\
\hline arterial embolism & 0.8 & 1.0 & 0 & \\
\hline other rare indications & 1.6 & 1.0 & 3.8 & \\
\hline
\end{tabular}

SD, standard deviation; VKA, vitamin K antagonist; AF, atrial fibrillation; CABG, coronary artery bypass graft

\section{Bleeding episodes}

In our study we found that 26 patients (20.2\%) suffered from 40 clinically relevant bleeding episodes during a mean follow-up of 15.5 months after inclusion. The mean time between inclusion (including testing) and bleeding was 9.8 months. The bleeding rates between male $(n=121)$ and female $(n=28)$ patients were comparable $(20 \%$ and $21 \%$ bleeding, respectively). Seventeen patients had one bleeding episode, six patients experienced two bleeding events, two patients had three bleeding episodes and one patient suffered five times from a clinically relevant bleeding. Patients experienced different types of bleeding (Table 1). In our population patients mainly experienced conjunctival eye bleeds. Four major bleeding episodes occurred during the follow-up period. Two patients experienced severe digestive tract bleedings and one patient suffered twice from severe nosebleeds.

\section{Thrombin generation}

Analyzing samples with whole blood CAT a significantly lower ETP and peak was found in the patients that suffered from bleeding compared to patients that did not have this adverse 
effect (Fig 1 A-B and Table 3). Differences in ETP and peak remained statistically significant in the presence of TM, although to a lesser degree (median [IQR] (CI) ETP: 134.9 [104.7-193] (126.4-169.9) versus 174.3 [129.5-222.9] (169.0-195.7), $p=0.009$; peak: 20.18 [13.74-30.39] (18.0-28.9) versus 27.72 [18.08-37.49] (27.1-37.0), $p=0.033(n=25$ and $n=102))$, in bleeding versus non-bleeding patients respectively). The lag time and time-to-peak did not differ significantly between bleeding and non-bleeding patients. A receiver operating curve (ROC) was constructed for ETP and peak determined in whole blood (Fig 1 C-D). Assessment of the area under the curve (AUC) of the ROC demonstrated that both ETP and peak were significantly associated (AUC (CI) ETP: 0.700 (0.584-0.816), $p=0.002(n=25)$ and AUC (CI) peak: $0.642(0.516-0.767), p=0.029(n=102)$, respectively) with the bleeding tendency.
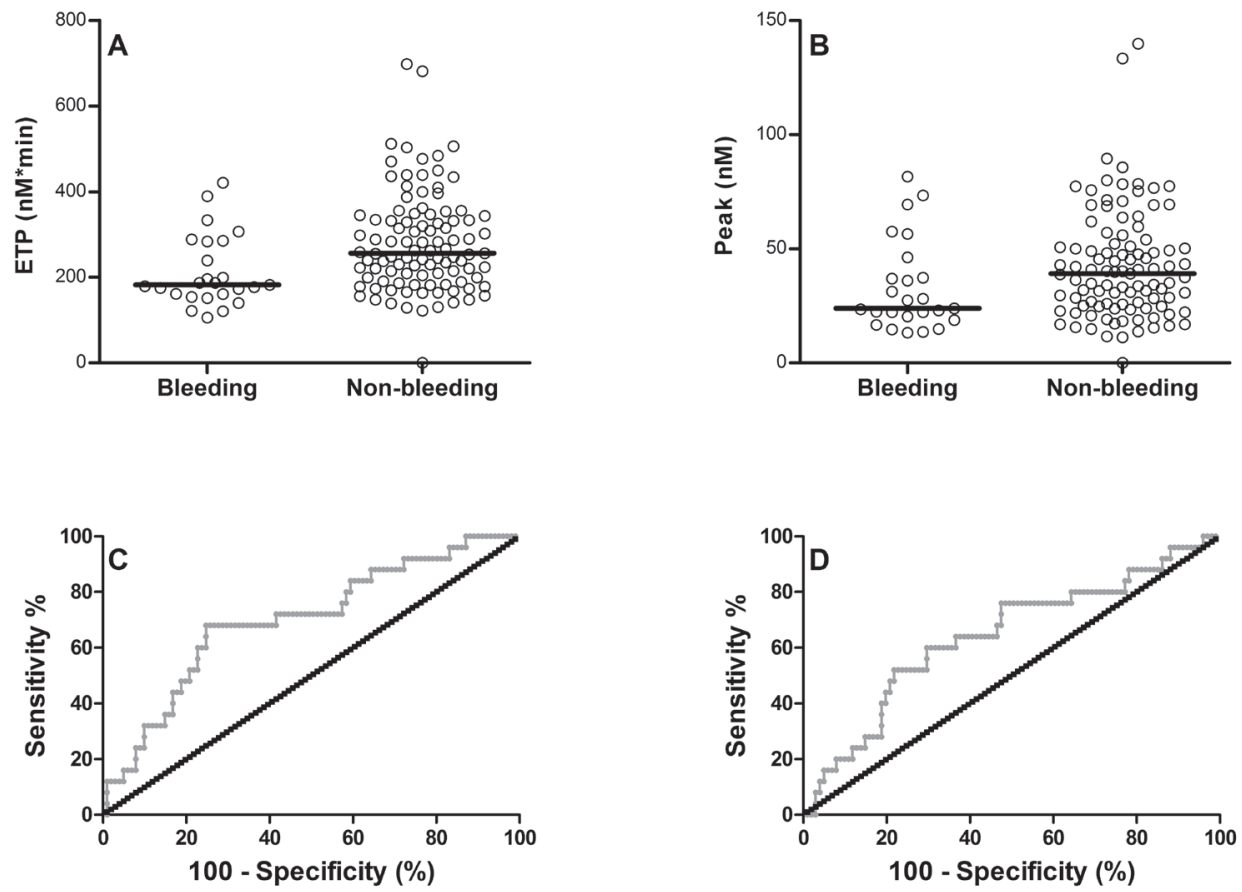

Figure 1. Whole blood CAT analysis of patients with and without bleeding symptoms.

(A) Difference in whole blood endogenous thrombin potential (ETP) $(p=0.002)$ in patients with and without bleeding. (B) Difference in whole blood peak $(p=0.029)$ in patients with and without bleeding. (C) Receiver operating curve $(R O C)$ of the ETP in whole blood thrombin generation (area under the curve $(A \cup C)=0.700, p=0.002$ ). (D) Receiver operating curve $(R O C)$ of the peak in whole blood thrombin generation $(A \cup C=0.642, p=0.029$ ). 


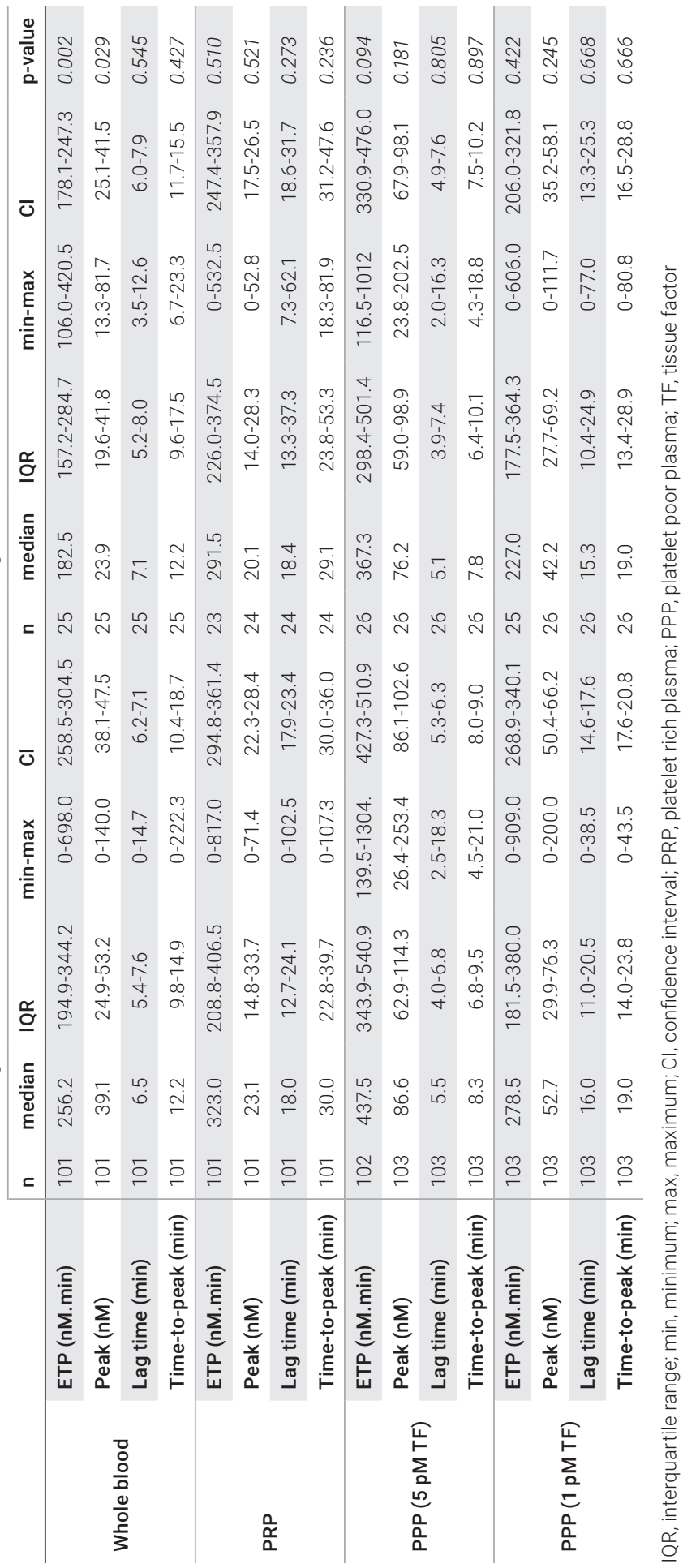


In plasma no significant differences were detected for the thrombin generation parameters between patients that suffered from bleeding and those without bleeding events, although a trend for a decreased ETP and peak height was observed in the bleeding population (Fig 2 and Table 3). Similarly, no significant differences were found in the lag time and time-to-peak between both populations. The addition of thrombomodulin did not change these results.
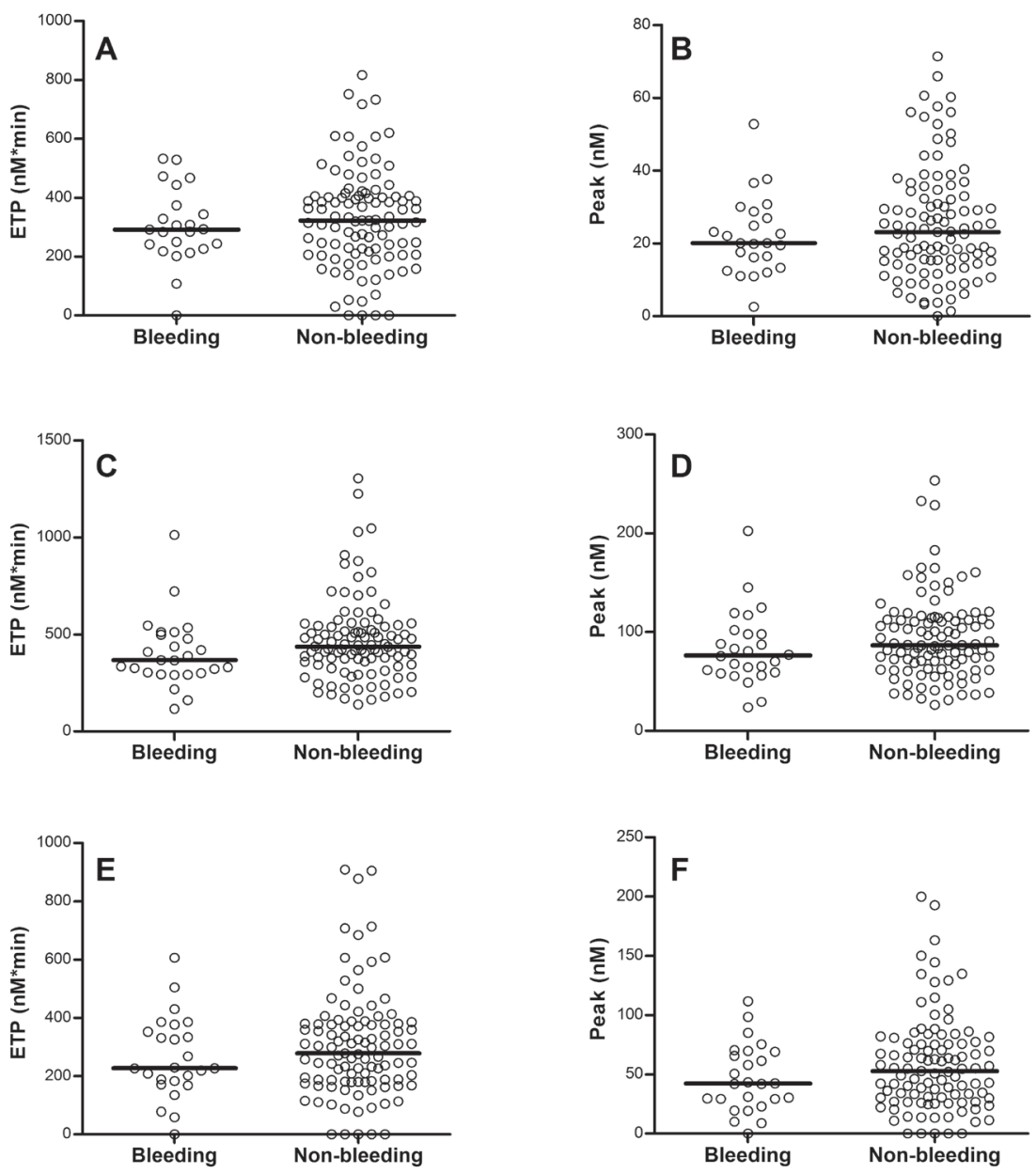

Figure 2. Plasma CAT analysis of patients with and without bleeding symptoms.

Analysis of the endogenous thrombin potential (ETP) and peak in patients with and without bleeding. (A) ETP in platelet rich plasma, (B) peak in platelet rich plasma, (C) ETP in platelet poor plasma at $5 \mathrm{pM}$ TF, (D) peak in platelet poor plasma at $5 \mathrm{pM} \mathrm{TF,} \mathrm{(E)} \mathrm{ETP} \mathrm{in} \mathrm{platelet} \mathrm{poor} \mathrm{plasma} \mathrm{at} 1 \mathrm{pM} \mathrm{TF,} \mathrm{(F)} \mathrm{peak} \mathrm{in} \mathrm{platelet} \mathrm{poor} \mathrm{plasma} \mathrm{at} 1 \mathrm{pM}$ TF. No significant differences were found for these parameters using the Mann Whitney $U$ test. Medians are indicated by lines. 
We assessed whether co-medication affecting platelets, could have an effect on the ETP and peak in whole blood. Twenty-nine patients used co-medication that affects platelets (P2Y12-inhibitors, acetylsalicylic acid and/or non-steroidal anti-inflammatory drugs) of which $12(41.4 \%)$ suffered from bleeding and 17 patients did not (S1 Table). From S1 Table it can also be calculated that $13.4 \%$ displayed bleeding when not using co-medication. We found that the use of these co-medications did not influence the ETP and peak measured in whole blood (S1 Table). Therefore our analyses were not corrected for this type of co-medication.

The parameters in whole blood CAT (1 pM TF) were compared to the parameters (ETP, peak, lag time and time-to-peak) in the plasma CAT (PRP at 1 pM TF and PPP at 1 and 5 pM TF). All thrombin generation parameters displayed a significant correlation between plasma and whole blood measurements and the highest correlation coefficients were established for the ETP and peak (S2 Table). The best correlation for the four parameters was found in PPP using 5 pM TF. Regarding the INR, similar correlations were observed with the whole blood CAT as compared to plasma CAT (inverse, hyperbolical correlation of ETP and peak with INR, linear correlation of lag time and time-to-peak with INR) (S3 Table).

\section{Additional analyses}

Bleeding events were not associated with a difference in age (median [IQR]: 70 [60-80] for both groups (Cl bleeding: 60-70 versus non-bleeding: 70-70), $p=0.4074(\mathrm{n}=26$ and $n=$ 103)). Patients with and without bleeding events did not significantly differ regarding the INR, hematocrit, hemoglobin levels, or fibrinogen concentration (S4 Supporting information). Differences in levels of other coagulation and anticoagulant factors were determined, but none of them reached statistical significance (S5 Table).

\section{HAS-BLED}

HAS-BLED scores in patients with bleeding episodes were significantly higher than in patients that did not bleed (median [IQR] (CI): 3 [2-3.25] (2.3-3.1) versus 2 [1-3] (1.9-2.3), $p=0.003(n=26$ and $n=103))($ Fig 3A). A ROC curve was constructed resulting in an AUC of 0.682 (Cl: 0.571-0.792) ( $p=0.004$ ) (Fig 3B). Additionally, the HAS-BLED score did not correlate to the whole blood ETP $(r=0.051, p=0.573)$ and peak $(r=0.075, p=0.401)$. 

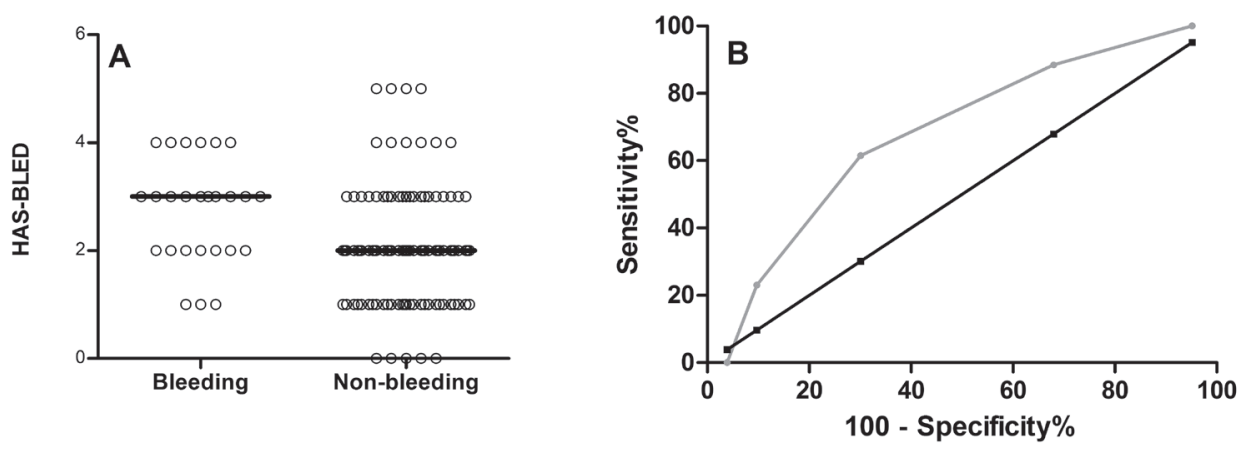

Figure 3. HAS-BLED analysis of bleeding and non-bleeding patients.

(A) Difference in HAS-BLED scores of patients with $(n=26)$ and without bleeding $(n=103, p=0.003)$. (B) Receiver operating curve (ROC) of the HAS-BLED scores (area under the curve $=0.682, p=0.004$ ).

\section{DISCUSSION}

Thrombin generation tests in plasma have been shown to provide a more complete overview of coagulation, since it encompasses both the extrinsic and intrinsic pathway. Clotting times (e.g. PT, aPTT) only include one of the pathways and measure the time until fibrin is formed, representing just $5 \%$ of the thrombin formation. ${ }^{11}$ It is known that an assessment of the complete thrombin generation is better related to clinical outcome.10,19-22 More recently, the whole blood CAT was developed, bringing thrombin generation one step closer to physiology paving the way for point of care thrombin generation assays. In this study, we provide a first clinical validation of this technique in patients using VKAs. Patients with and without clinically relevant bleeding episodes significantly differed in whole blood CAT ETP and peak. As expected the INR, which is the standard follow up test for patients taking VKAs, was not predictive for bleeding on the long term; for the treatment of patients is adjusted to the measured INR.

In a population of patients using VKAs the whole blood CAT suggests to be the first laboratory test that is related to a bleeding risk in a prospective set-up. The AUC of ROC is a measure for the discrimination of a test/score between patient with and without the disease. The ETP and peak in whole blood have an AUC of ROC of 0.700 and 0.642 , respectively and therefore particularly the ETP can be considered as a fair predictor of bleeding. Although these parameters cannot fully discriminate between bleeding and non-bleeding patients, their discriminative value is at least comparable to that of the HASBLED score. In contrast, 
the plasma-based CAT did not discriminate between the bleeding and non-bleeding patients. Other studies showed that plasma CAT was indicative of bleeding in different patient populations. ${ }^{19,23,24}$ However, this finding could not be repeated in our study. The presence of platelets alone (PRP) did not improve the outcome of the test considering discrimination between patients with and without bleeding. This is in accordance to earlier findings indicating that recurrent bleeding in patients with a stable INR cannot be explained by changes in platelets or von Willebrand factor function. ${ }^{25}$ In contrast to plasma CAT, whole blood CAT includes erythrocytes that can directly contribute to thrombin generation, e.g. as a source of procoagulant phospholipids on the cell membrane. Interplay between the coagulation system and these red blood cells may provide an explanation that whole blood CAT, but not plasma CAT enables discrimination between bleeding and non-bleeding patients in patients on VKAs. Earlier studies by Whelihan et al. show that a sub-fraction of red blood cells express phosphatidylserine and this might serve as a surface for thrombin and other phospholipid-bound coagulation factors. ${ }^{26}$ Apart from erythrocytes, whole blood differs from plasma in the presence of leukocytes. The release of TF by these cells (in particular monocytes) may also contribute to the differential activation of coagulation in bleeding versus non-bleeding patients. However, the exact mechanism resulting in an improved discrimination of whole blood thrombin generation between patients with and without bleeding episodes needs further research.

Bleedings that occurred during the study were mostly spontaneous, minor bleedings; none were caused by surgical intervention or major trauma. Although this increases the likelihood of similar etiology, the bleedings in this study population can still result from multiple factors, e.g. VKA treatment, co-medication, the coagulation system (e.g. clotting factors), age, gender or TTR. Co-medication is one of the known factors that can increase the risk of bleeding. 5,27 In our population we found that $41.4 \%$ of the patients who were taking co-medication affecting platelets suffered from bleeding, whereas this was only $13.4 \%$ in patients that did not use co-medication. In this study whole blood ETP and peak were not influenced by the use of co-medication. This confirms previously reported results suggesting that the presence or absence of platelets and by extension platelet agonists or antagonists, had no effect on thrombin generation in whole blood. ${ }^{13}$ Moreover, we detected no differences between bleeding and non-bleeding patients in PRP. Therefore our analyses were not corrected for the use of co-medications. Furthermore, clotting factor deficiencies or increased levels of natural anticoagulants could result in an increased bleeding risk. However, 
both clotting factor and anticoagulant factor levels proved to be similar in the bleeding and non-bleeding patients. Additionally, an increased age or difference in gender distribution may confer an increased risk for bleeding. 4,28,29 Yet in our study no significant difference in age or gender distribution was found between the patients with and without bleeding episodes. The TTR (which is also included in the HAS-BLED score) was lower in patients with bleeding. It is known that patients with a low TTR are at higher risk of complications (either bleeding or thrombosis). ${ }^{30} \mathrm{~A}$ significantly higher HAS-BLED score was detected in patients with bleeding events compared to those without. The ROC AUC of the HAS-BLED score was comparable to that of the whole blood ETP and peak. The HAS-BLED score was developed for AF patients; however other patient groups taking VKAs have comparable risk factors for bleeding. In the present study $72 \%$ of the population was treated for $A F$ and $28 \%$ of the patients had other indications. Even within the latter group, in spite of its small number, a significant difference in HAS-BLED scores was found between the bleeding and the non-bleeding group, indicating that the HAS-BLED could be of value in these patients. Since bleeding may be provoked by many different factors, it seems unlikely that one single test would be able to predict a patients bleeding risk. Within this reasoning, a combination of laboratory tests with existing bleeding scores, may lead to a more accurate prediction of the bleeding risk. On the other hand, it could also be suggested to include the whole blood CAT ETP and peak in the HAS-BLED score, since this was the coagulation assay with the best clinical association. The INR, which is currently incorporated in the HAS-BLED score, did not prospectively discriminate between bleeding and non-bleeding patients. Additionally, there was no correlation between whole blood TG parameters and the HAS-BLED score. Therefore, we speculate that the predictive value of the HAS-BLED could be improved by replacing the INR by whole blood CAT parameters. This would then open the possibility to also use this score in assessing the bleeding risk for patients using DOACs. We expect this might be important in the future with the increasing use of DOACs.

Inevitably, this study had some limitations. Firstly, the observed risk associations are with all clinically relevant bleeding complications, whereas it could be argued that only major bleeding complications matter. Previous studies showed that patients with minor bleeding events are at increased risk (>2.5-fold) for major bleedings. ${ }^{31,32}$ Therefore, we believe that the inclusion of minor, clinically relevant bleedings is a rational and clinically relevant choice. Secondly, it can be argued whether this study featured a large enough sample size to discover a relevant effect of bleeding predisposition on thrombin generation, but in accordance with 
our power calculation, the observed rate of bleeding complications allows for drawing conclusions based on the presented data. It is obvious that in order to assess the utility of whole blood CAT testing in patients on anticoagulation a focus on major bleeding complications will be important, which warrants repetition in a larger study to corroborate our findings. Finally, it is known that some biomarkers and risk factors associated with bleeding have also been linked to stroke and systemic thromboembolism. Therefore, it would be interesting to explore this topic. Unfortunately we were unable to do so because there were no episodes of recurrent VTE or stroke during the follow-up period.

In conclusion, clinically relevant bleeding in patients taking VKAs in our study is associated with a diminished whole blood ETP and peak, but not with INR. Consequently, whole blood ETP and peak could be of value in assessing patients who suffer from recurrent bleeding with an INR in therapeutic range. In our population an augmented HAS-BLED score was associated with bleeding as well. Implementing whole blood CAT might improve the sensitivity of bleeding scores such as the HAS-BLED, enabling further tailoring of therapy for individual patients.

\section{ACKNOWLEDGMENTS}

The authors would like to thank Hilde Kelchtermans for critically reading the manuscript and the employees of the Maastricht Anticoagulation Clinic for their assistance with patient inclusion and blood collection. 


\section{REFERENCES}

1. Ansell J, Hirsh J, Hylek E, Jacobson A, Crowther M, Palareti G. Pharmacology and management of the vitamin K antagonists: American College of Chest Physicians Evidence-Based Clinical Practice Guidelines (8th Edition). Chest. 2008;133(6 Suppl):160s-198s

2. Halbritter K, Beyer-Westendorf J, Nowotny J, Pannach S, Kuhlisch E, Schellong SM. Hospitalization for vitamin-K-antagonist-related bleeding: treatment patterns and outcome. Journal of thrombosis and haemostasis : JTH. 2013;11(4):651-659.

3. Connolly SJ, Ezekowitz MD, Yusuf S, et al. Dabigatran versus warfarin in patients with atrial fibrillation. The New England journal of medicine. 2009;361(12):1139-1151.

4. Levine MN, Raskob G, Beyth RJ, Kearon C, Schulman S. Hemorrhagic complications of anticoagulant treatment: the Seventh ACCP Conference on Antithrombotic and Thrombolytic Therapy. Chest. 2004;126(3 Suppl):287s-310s.

5. Hallas J, Dall M, Andries A, et al. Use of single and combined antithrombotic therapy and risk of serious upper gastrointestinal bleeding: population based case-control study. BMJ (Clinical research ed). 2006;333(7571):726.

6. Gallego P, Roldan V, Torregrosa JM, et al. Relation of the HAS-BLED bleeding risk score to major bleeding, cardiovascular events, and mortality in anticoagulated patients with atrial fibrillation. Circulation Arrhythmia and electrophysiology. 2012;5(2):312-318.

7. Apostolakis S, Lane DA, Buller H, Lip GY. Comparison of the CHADS2, CHA2DS2-VASc and HAS-BLED scores for the prediction of clinically relevant bleeding in anticoagulated patients with atrial fibrillation: the AMADEUS trial. Thrombosis and haemostasis. 2013;110(5):1074-1079.

8. Lane DA, Lip GY. Use of the CHA(2)DS(2)-VASc and HAS-BLED scores to aid decision making for thromboprophylaxis in nonvalvular atrial fibrillation. Circulation. 2012;126(7):860-865.

9. Olesen JB, Lip GY, Hansen PR, et al. Bleeding risk in 'real world' patients with atrial fibrillation: comparison of two established bleeding prediction schemes in a nationwide cohort. Journal of thrombosis and haemostasis : JTH. 2011;9(8):1460-1467.

10. Hemker HC, Giesen P, AIDieri R, et al. The calibrated automated thrombogram (CAT): a universal routine test for hyper- and hypocoagulability. Pathophysiology of haemostasis and thrombosis. 2002;32(5-6):249-253.

11. Al Dieri R, de Laat B, Hemker HC. Thrombin generation: what have we learned? Blood Rev. 2012;26(5):197-203.

12. Bloemen S, Hemker HC, Al Dieri R. Large inter-individual variation of the pharmacodynamic effect of anticoagulant drugs on thrombin generation. Haematologica. 2013;98(4):549-554.

13. Ninivaggi M, Apitz-Castro R, Dargaud Y, de Laat B, Hemker HC, Lindhout T. Whole-blood thrombin generation monitored with a calibrated automated thrombogram-based assay. Clinical chemistry. 2012;58(8):1252-1259.

14. Schulman S, Kearon C. Definition of major bleeding in clinical investigations of antihemostatic medicinal products in non-surgical patients. Journal of thrombosis and haemostasis : JTH. 2005;3(4):692-694.

15. Hemker HC, Giesen P, Al Dieri R, et al. Calibrated automated thrombin generation measurement in clotting plasma. Pathophysiology of haemostasis and thrombosis. 2003;33(1):4-15.

16. Coen Hemker H, Hemker PW, Al Dieri R. The technique of measuring thrombin generation with fluorescent substrates: 4. The H-transform, a mathematical procedure to obtain thrombin concentrations without external calibration. Thrombosis and haemostasis. 2009;101(1):171-177.

17. Hemker HC, Kremers R. Data management in thrombin generation. Thrombosis research. 2013;131(1):3-11.

18. Pisters R, Lane DA, Nieuwlaat R, de Vos CB, Crijns HJ, Lip GY. A novel user-friendly score (HAS-BLED) to assess 1-year risk of major bleeding in patients with atrial fibrillation: the Euro Heart Survey. Chest. 2010;138(5):1093-1100. 
19. Bosch YP, Al Dieri R, ten Cate $H$, et al. Measurement of thrombin generation intra-operatively and its association with bleeding tendency after cardiac surgery. Thrombosis research. 2014;133(3):488-494.

20. Al Dieri R, Peyvandi F, Santagostino E, et al. The thrombogram in rare inherited coagulation disorders: its relation to clinical bleeding. Thrombosis and haemostasis. 2002;88(4):576-582.

21. Dargaud $Y$, Beguin $S$, Lienhart $A$, et al. Evaluation of thrombin generating capacity in plasma from patients with haemophilia A and B. Thrombosis and haemostasis. 2005;93(3):475-480.

22. van Hylckama Vlieg A, Christiansen SC, Luddington R, Cannegieter SC, Rosendaal FR, Baglin TP. Elevated endogenous thrombin potential is associated with an increased risk of a first deep venous thrombosis but not with the risk of recurrence. $\mathrm{Br} J$ Haematol. 2007;138(6):769-774.

23. Dargaud $Y$, Hoffman $M$, Lefrapper $L$, et al. Bleeding risk in warfarinized patients with a therapeutic international normalized ratio: the effect of low factor IX levels. Journal of thrombosis and haemostasis : JTH. 2013;11(6):1043-1052.

24. Luna-Zaizar H, Gonzalez-Moncada Al, Padilla-Lopez EL, et al. Thrombin generation and international normalized ratio in inherited thrombophilia patients receiving thromboprophylactic therapy. Thrombosis research. 2015;136(6):1291-1298.

25. van der Meijden PE, Bouman AC, Feijge MA, et al. Platelet dysfunction in thrombosis patients treated with vitamin K antagonists and recurrent bleeding. PloS one. 2013;8(5):e64112.

26. Whelihan MF, Mann KG. The role of the red cell membrane in thrombin generation. Thrombosis research. 2013;131(5):377-382

27. Rothberg MB, Celestin C, Fiore LD, Lawler E, Cook JR. Warfarin plus aspirin after myocardial infarction or the acute coronary syndrome: meta-analysis with estimates of risk and benefit. Annals of internal medicine. 2005;143(4):241-250.

28. Lip GY, Clementy N, Pericart L, Banerjee A, Fauchier L. Stroke and major bleeding risk in elderly patients aged >/=75 years with atrial fibrillation: the Loire Valley atrial fibrillation project. Stroke. 2015;46(1):143-150.

29. Poli D, Antonucci E, Testa S, Tosetto A, Ageno W, Palareti G. Bleeding risk in very old patients on vitamin $K$ antagonist treatment: results of a prospective collaborative study on elderly patients followed by Italian Centres for Anticoagulation. Circulation. 2011;124(7):824-829.

30. Sanden P, Renlund H, Svensson PJ, Sjalander A. Bleeding complications and mortality in warfarin-treated VTE patients, dependence of INR variability and iTTR. Thrombosis and haemostasis. 2017;117(1):27-32.

31. van Rein N, le Cessie S, van Vliet IP, et al. Increased risk of major bleeding after a minor bleed during treatment with vitamin $\mathrm{K}$ antagonists is determined by fixed common risk factors. Journal of thrombosis and haemostasis : JTH. 2016;14(5):948-952.

32. Veeger NJ, Piersma-Wichers M, Meijer K, Hillege HL. Minor bleeds alert for subsequent major bleeding in patients using vitamin K antagonists. Br J Haematol. 2011;153(4):508-514. 


\section{SUPPLEMENTAL INFORMATION}

S1 Table. The use of co-medications did not influence whole blood ETP and peak.

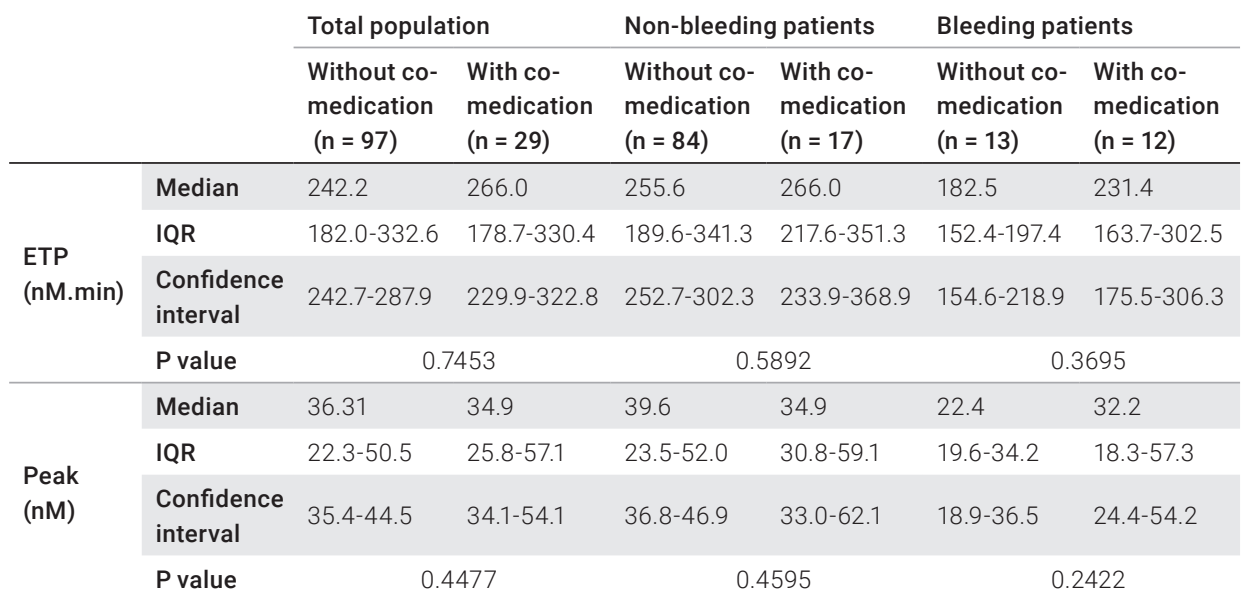

$I Q R$, interquartile range

S2 Table. Correlation of whole blood CAT with plasma CAT,

\begin{tabular}{lllll} 
R-value & ETP $(\mathrm{nM} . \mathrm{min})$ & Peak $(\mathrm{nM})$ & Lag time $(\mathrm{min})$ & Time-to-peak $(\mathrm{min})$ \\
\hline PRP & 0.593 & 0.651 & 0.300 & 0.449 \\
& $p=0.000$ & $p=0.000$ & $p=0.001$ & $p=0.000$ \\
PPP (5 pM TF) & 0.675 & 0.815 & 0.426 & 0.510 \\
& $p=0.000$ & $p=0.000$ & $p=0.000$ & $p=0.000$ \\
PPP (1 pM TF) & 0.613 & 0.767 & 0.252 & 0.506 \\
& $p=0.000$ & $p=0.000$ & $p=0.004$ & $p=0.000$
\end{tabular}

PRP, platelet rich plasma; PPP, platelet poor plasma; TF, tissue factor; ETP, endogenous thrombin potential

S3 Table. Correlation of INR with CAT parameters.

\begin{tabular}{lllll} 
R-value & ETP $(\mathrm{nM} \cdot \mathbf{m i n})$ & Peak $(\mathrm{nM})$ & Lag time $(\mathrm{min})$ & Time-to-peak $(\mathrm{min})$ \\
\hline Whole blood & -0.456 & -0.720 & 0.504 & 0.622 \\
\hline PRP & $p=0.000$ & $p=0.000$ & $p=0.000$ & $p=0.000$ \\
& -0.626 & -0.653 & 0.581 & 0.605 \\
\hline PPP (5 pM TF) & -0.748 & $p=0.000$ & $p=0.000$ & $p=0.000$ \\
& $p=0.000$ & -0.782 & 0.466 & 0.489 \\
PPP (1 pM TF) & -0.784 & $p=0.000$ & $p=0.000$ & $p=0.000$ \\
& $p=0.000$ & -0.768 & 0.384 & 0.377 \\
\hline
\end{tabular}

PRP, platelet rich plasma; PPP, platelet poor plasma; TF, tissue factor; ETP, endogenous thrombin potential 
S4 Supporting information. Medians with ranges parameters which are known to be related with bleeding.

\begin{tabular}{|c|c|c|c|c|c|c|c|c|c|c|c|}
\hline & \multicolumn{5}{|c|}{ Non-bleeding } & \multicolumn{6}{|c|}{ Bleeding } \\
\hline INR & 103 & 2.9 & 2.3-3.7 & $1.1-5.7$ & $2.8-3.1$ & 26 & 3.0 & $2.3-3.5$ & $1.4-5.1$ & $2.6-3.3$ & 0.874 \\
\hline Hematocrit & 103 & 0.38 & $0.36-0.42$ & $0.24-0.47$ & $0.38-0.40$ & 26 & 0.38 & $0.35-0.40$ & $0.25-0.46$ & $0.35-0.39$ & 0.232 \\
\hline Hemoglobin & 103 & 7.6 & $7.1-8.2$ & $4.6-9.9$ & $7.5-7.8$ & 26 & 7.4 & $6.6-7.9$ & $5.2-9.1$ & $6.9-7.7$ & 0.112 \\
\hline
\end{tabular}

INR, international normalized ratio; IQR, interquartile range; $\mathrm{Cl}$, confidence interval
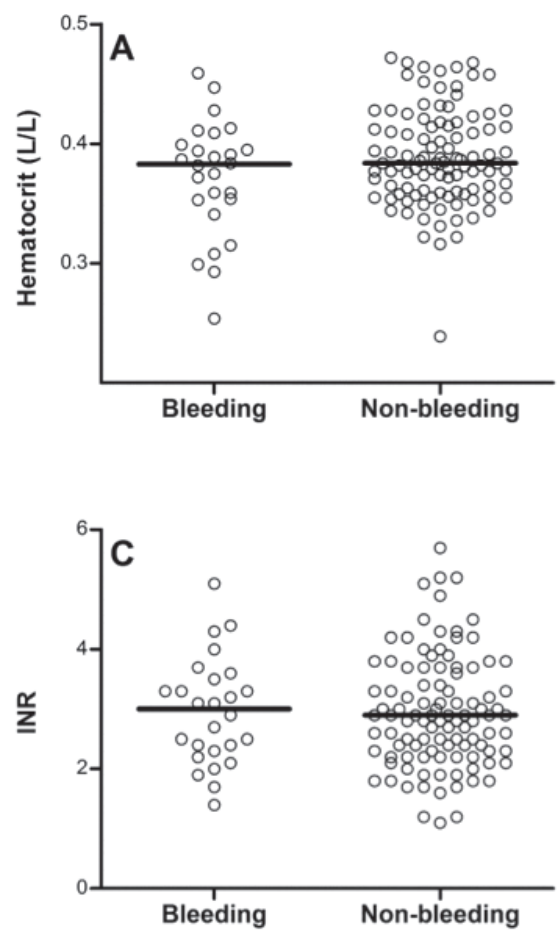
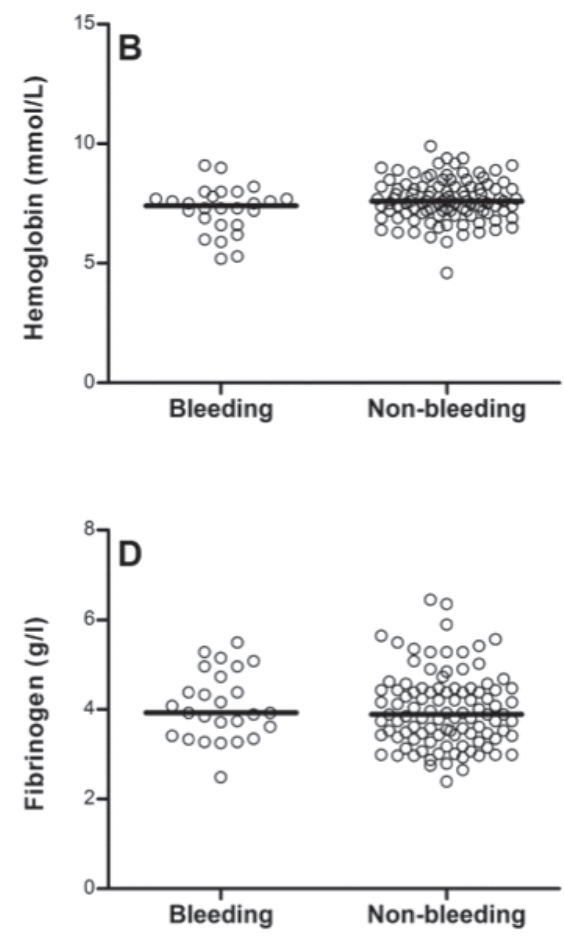

S5 Figure 1. Hematocrit, hemoglobin, international normalized ratio (INR) and fibrinogen determinations in patients with and without bleeding symptoms.

Determinations of $(A)$ hematocrit $(p=0.232)$, (B) hemoglobin $(p=0.112)$, (C) INR ( $p=0.874)$ and (D) fibrinogen $(p=$ 0.540 ) in patients with bleeding compared to without bleeding. 
S5 Table. Medians with interquartile ranges of coagulation factor determinations.

\begin{tabular}{|c|c|c|c|c|c|c|}
\hline & \multicolumn{2}{|l|}{ Prothrombin } & \multicolumn{2}{|l|}{ Factor V } & \multicolumn{2}{|l|}{ Factor VII } \\
\hline & Bleeding & Non-bleeding & Bleeding & Non-bleeding & Bleeding & Non-bleeding \\
\hline $\mathrm{n}$ & 26 & 129 & 26 & 128 & 26 & 127 \\
\hline Median & 19 & 23 & 101.5 & 104 & 17 & 15 \\
\hline [IQR 25\%-75\%] & [15.8-27.3] & [16.5-29] & [89.8-115.3] & [91-115] & [12-24.3] & [10-23] \\
\hline $\mathrm{Cl}$ & $18.4-25.1$ & $22.4-26.0$ & $95.1-112.1$ & 101.0-107.4 & $14.2-25.0$ & $16.3-20.8$ \\
\hline \multirow[t]{3}{*}{ P-value } & \multicolumn{2}{|l|}{0.304} & \multicolumn{2}{|l|}{0.806} & \multicolumn{2}{|l|}{0.502} \\
\hline & \multicolumn{2}{|l|}{ Factor VIII } & \multicolumn{2}{|l|}{ Factor IX } & \multicolumn{2}{|l|}{ Factor X } \\
\hline & Bleeding & Non-bleeding & Bleeding & Non-bleeding & Bleeding & Non-bleeding \\
\hline $\mathrm{n}$ & 17 & 105 & 24 & 124 & 26 & 128 \\
\hline Median & 174 & 179 & 33 & 36 & 11 & 13 \\
\hline [IQR 25\%-75\%] & [135-234] & [141-218.5] & [24.3-41.8] & [26-46.8] & [8-14.8] & [9-16] \\
\hline $\mathrm{Cl}$ & 152.0-222.- & 173.2-195.7 & $29.3-42.9$ & $36.1-42.9$ & $10.3-14.7$ & $12.9-15.4$ \\
\hline \multirow[t]{3}{*}{ P-value } & \multicolumn{2}{|l|}{0.968} & \multicolumn{2}{|l|}{0.357} & \multicolumn{2}{|l|}{0.197} \\
\hline & \multicolumn{2}{|l|}{ Protein C } & \multicolumn{2}{|l|}{ Protein S } & \multicolumn{2}{|l|}{ Antithrombin } \\
\hline & Bleeding & Non-bleeding & Bleeding & Non-bleeding & Bleeding & Non-bleeding \\
\hline $\mathrm{n}$ & 23 & 101 & 15 & 99 & 26 & 129 \\
\hline Median & 10 & 11 & 35 & 36 & 112 & 108 \\
\hline [IQR 25\%-75\%] & [5-23] & [5.3-23] & [25-40] & [27-42] & [104.5-118.8] & [102-116] \\
\hline $\mathrm{Cl}$ & $9.1-21.6$ & $13.0-19.2$ & $26.9-41.3$ & 34.3-39.7 & $107.8-116.4$ & 106.9-111.4 \\
\hline P-value & 0.953 & & 0.443 & & 0.235 & \\
\hline
\end{tabular}

$\mathrm{IQR}$, interquartile range; $\mathrm{Cl}$, confidence interval 


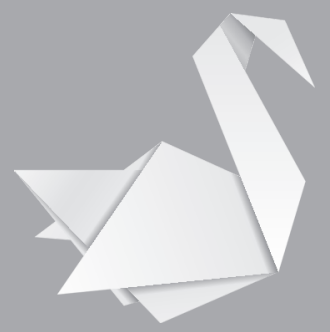




\section{CHAPTER}

Calibrated automated thrombinography (CAT), a tool to identify patients at risk of bleeding during anticoagulant therapy?

A systematic review

Suzanne Zwaveling

Saartje Bloemen

Bas de Laat

Hugo ten Cate

Arina ten Cate-Hoek

TH open 2018;2:e291-e302. 


\section{ABSTRACT}

\section{Background}

Bleeding is a feared adverse event during anticoagulant treatment. In patients on vitamin-K-antagonists, most bleedings occur with the international normalized ratio (INR) in the therapeutic range. Currently, identification of high-risk patients via laboratory methods is not reliable. In this systematic review we assed the ability of calibrated automated thrombin generation (CAT-TG) to predict bleeding in patients on anticoagulant treatment.

\section{Methods}

A systematic search was executed in three databases: Medline, Embase, and Cochrane.

\section{Results}

Seven studies were included; two were of good methodological quality. One study showed that patients on warfarin with INRs in range (2-3) admitted for haemorrhage ( $\mathrm{n}=28)$, had lower CAT-TG levels (endogenous thrombin potential (ETP) 333+/-89nM.min ${ }^{-1}$ ) than patients on warfarin admitted for other reasons (ETP 436+/-207nM. min $^{-1} ; \mathrm{P}<0.001$ ). Another study found no difference in ETP or Peak levels between bleeding and non-bleeding patients in PPP or PRP. When measured in whole blood, both levels were significantly lower in patients with bleeding compared to non-bleeding patients (median [interquartile range (IQR)] ETP: 182.5 [157.2-284,7nM. min-1] vs. median [IQR] ETP: 256.2 [194.9-344.2nM.min-1 ${ }^{-1}$; $(P<0.001)$ and median [IQR] peak 23.9 [19.6-41.8nM] vs. median [IQR] peak 39.1 [24.9-53.2nM]; $(\mathrm{P}<0.05)$. From the remaining studies, four suggested that CAT-TG is more sensitive in detecting haemostatic abnormalities than INR and one paper found ETP and INR to be equally useful. However, insufficient data were provided to validate these conclusions.

\section{Conclusion}

Studies investigating the direct association between decreased CAT-TG values and haemorrhagic events are rare. Therefore, the clinical consequences of low CAT-TG values remains to be further investigated.

\section{Highlights:}

- In patients using VKAs an association between decreased calibrated automated thrombin generation and bleeding was found, however only few studies have been performed

- Patients with INR in the same range, can have varying thrombin generation values

- The association between thrombin generation and bleeding in patients using DOACs remains to be elucidated 


\section{INTRODUCTION}

Thromboembolic disease is a major cause of morbidity and mortality worldwide. The treatment of thromboembolism relies on anticoagulant drugs of which direct oral anticoagulants (DOACs) and vitamin-K-antagonists (VKAs) are the most widely prescribed. Although anticoagulant therapy has proven to be effective since many years, it is associated with serious adverse effects. Major bleeding is a feared, but also prevalent complication with an incidence of 1-3\% annually. Patients on VKAs are monitored by measuring the prothrombin time (PT), which is standardized to the international normalized ratio (INR). The INR is used to adjust therapy towards therapeutic levels order to minimize the risk of bleeding. ${ }^{2}$ Nevertheless, clinical studies have shown that most bleeding complications occur within a therapeutic INR range. ${ }^{3-5}$ Rates of major bleedings between $3.09 \%$ and $3.4 \%$ have been described in patients using warfarin with a time in therapeutic range (TTR) of $66 \%$; for cerebral haemorrhage these numbers are approximately between $0.38 \%$ and $0.7 \%{ }^{6-8}$ For DOACs the rates of major bleedings lie between $2.13 \%$ and $3.11 \%$, and rates of cerebral haemorrhage are between $0.1 \%$ and $0.5 \% .^{6-8}$

Other than INR assessment, which at least helps to reduce the risk of bleeding in patients using VKAs, no other laboratory test has been helpful in limiting bleeding risk during anticoagulant therapy. The predictive value for bleeding of the PT and the activated partial thromboplastin time (aPTT) has been investigated in several studies, which all found poor correlations between these laboratory tests and haemorrhagic events. ${ }^{9-11}$ Furthermore, studies have shown that the PT and aPTT are unsuitable to measure the effect of DOACs. In contrast, global coagulation tests, like CAT-TG, are able to capture more aspects of the coagulation system, and could potentially be beneficial in assessing the total anticoagulant effects. By this reasoning, some studies indeed suggested added value of CAT-TG testing in relation to bleeding outcome ${ }^{12}$, or for monitoring of patients on anticoagulant therapy. ${ }^{13}$ CAT-TG is able to show the anticoagulant effect of many, possibly all, anticoagulants including DOACs. Multiple studies have previously found that patients who are treated with VKAs have a diminished amount of thrombin that is formed over time (low endogenous thrombin potential (ETP)), a lower maximum of thrombin that is formed (low Peak height), and a postponed start of coagulation (prolonged lag time) compared to healthy controls. ${ }^{14,15}$

We hypothesized that patients that are at risk of bleeding during anticoagulant therapy would have even lower CAT-TG values than patients using anticoagulants who are less at risk of bleeding. In this systematic review we evaluate whether CAT-TG can detect the risk of bleeding in patients on oral anticoagulant treatment, and as such, would be of potential clinical value. 


\section{METHODS}

\section{Data sources and searches}

We performed a systemic search for studies evaluating the use of CAT-TG to predict bleeding in patients on anticoagulant therapy in three databases (Medline, Embase en Cochrane). The date of the search performed in Medline was May 18 ${ }^{\text {th }} 2017$. On the $22^{\text {th }}$ of May 2017 we systematically searched the Embase and Cochrane databases. No restrictions or filters with regard to language, publication date and age were applied. The search strategy was refined using keywords of references found in a pilot search and after manual review of reference lists. The keywords included synonyms for "thrombin generation", "anticoagulant therapy" and "bleeding" as outcome (see Supplementary Appendix 1 for the complete search strategy). If a paper was eligible for full-text reading but could not be retrieved, attempts were made to retrieve the article by searching other libraries or contacting authors. Authors were also contacted in case reports were only available as conference abstracts. The results of the database searches were supplemented by manual review of a reference list of papers that met the inclusion criteria. Duplicate articles where filtered manually.

\section{Study selection}

Two reviewers independently screened abstracts between May $22^{\text {the }} 2017$ until July 2017 and selected papers for eligibility based on pre-defined in- and exclusion criteria. A third reviewer was consulted to agree on the final selection and to resolve any discrepancies between the first two reviewers. For a complete overview of the selection procedures see figure 1. We included studies when fulfilling the following criteria: 1) research was performed in patients using oral or parenteral anticoagulant treatment for more than three months; 2) CAT-TG was measured using a calibrated automated global TG test and the most common parameters: lag time (the time until the first thrombin is formed), peak (the maximum amount of thrombin that is formed), time-to-peak (time until the maximum is reached) and the endogenous thrombin potential (ETP) (the total amount of thrombin that can be formed over time); 3) there was a clear description of the method of CAT-TG, e.g. noting the amount of tissue factor (TF), the use of corn trypsin inhibitor (CTI), thrombomodulin (TM) or activated protein $\mathrm{C}$ (APC), the use of phospholipids and the characteristics of the sample material (platelet poor plasma (PPP), platelet rich plasma (PRP) or whole blood) and 4) the outcome of CAT-TG was related to bleeding. Bleedings should have occurred spontaneously, i.e. not by a procedure (e.g. post-operative bleeding or a punch biopsy). Additionally, bleeding episodes should have 
been well documented and described. When a study cohort was described by more than one article, we only included the original data. Studies were excluded if they 1) consisted of non-original data (e.g. reviews, guidelines, comments), 2) were not written in English, 3) were performed in patients with a known bleeding disorder (e.g. haemophilia), severe liver cirrhosis or liver failure, cancer, or in paediatric patients, 4) were animal studies, 5) did not use the calibrated automated thrombinography (CAT)-method as automated global thrombin generation test but e.g. the Technoclone method, measured prothrombin fragment F1 and F2, or thrombin-anti thrombin complexes or 6 ) did not have "spontaneous clinical bleeding" as the outcome (e.g. post-operative bleeding or punch biopsy).

\section{Data extraction and quality assessment}

The included studies were reviewed in duplicate and data was extracted using a standardized form. The extracted data included author, journal, year of publication, study design, clinical setting, number of patients, patient characteristics including the type of anticoagulant, the method of TG, other types of test used for comparison, bleeding events, follow-up, statistical analysis and results. It was intended to construct $2 \times 2$ tables where possible, for the patients with a high/low risk of bleeding, using the extracted numbers of true and false positive as well as negative results according the TG test. Methodological quality of studies was assessed using the Newcastle-Ottawa quality assessment scale for cohort studies and case-control studies. To be able to classify the quality of the articles we created a table in which we defined the amount of stars. Both are described in the Supplementary Appendix. ${ }^{16}$ 


\section{RESULTS}

\section{Search results}

The database search yielded 1698 papers in total; 674 papers were retrieved from Medline, 818 from Embase and 206 from Cochrane (Figure 1). 1387 papers were excluded based on title or abstract. Eighty-seven papers remained eligible and were evaluated based on full text. The majority of studies were not performed in patients on long-term anticoagulant treatment and/or did not assess the direct relation between CAT-TG and clinical bleeding in the absence of an intervention and were therefore excluded $(n=50)$. For example 31 of these studies assessed TG as a tool to investigate the reversal of anticoagulation. The examined drugs in these studies were often DOACs and tests were mostly performed in healthy subjects. These studies used the normalisation of coagulation tests (such as CAT-TG) as an indication that coagulation was restored, but did not investigate the relation with clinical bleeding. ${ }^{13,17-46}$ Eight studies investigated the role of TG in prediction of bleeding after cardiac surgery. ${ }^{47-54}$ These studies were excluded because all patients underwent surgical intervention; therefore, the outcome was not compatible with spontaneous haemorrhage $(n=8)$. We did not find any studies investigating DOACs and the direct relation between TG and clinical bleeding. Finally, 7 papers were included. All papers studied the value of CAT-TG to evaluate a bleeding risk in patients using VKAs and two of these are currently unpublished. We approached the authors, but unfortunately no additional information could be retrieved. 


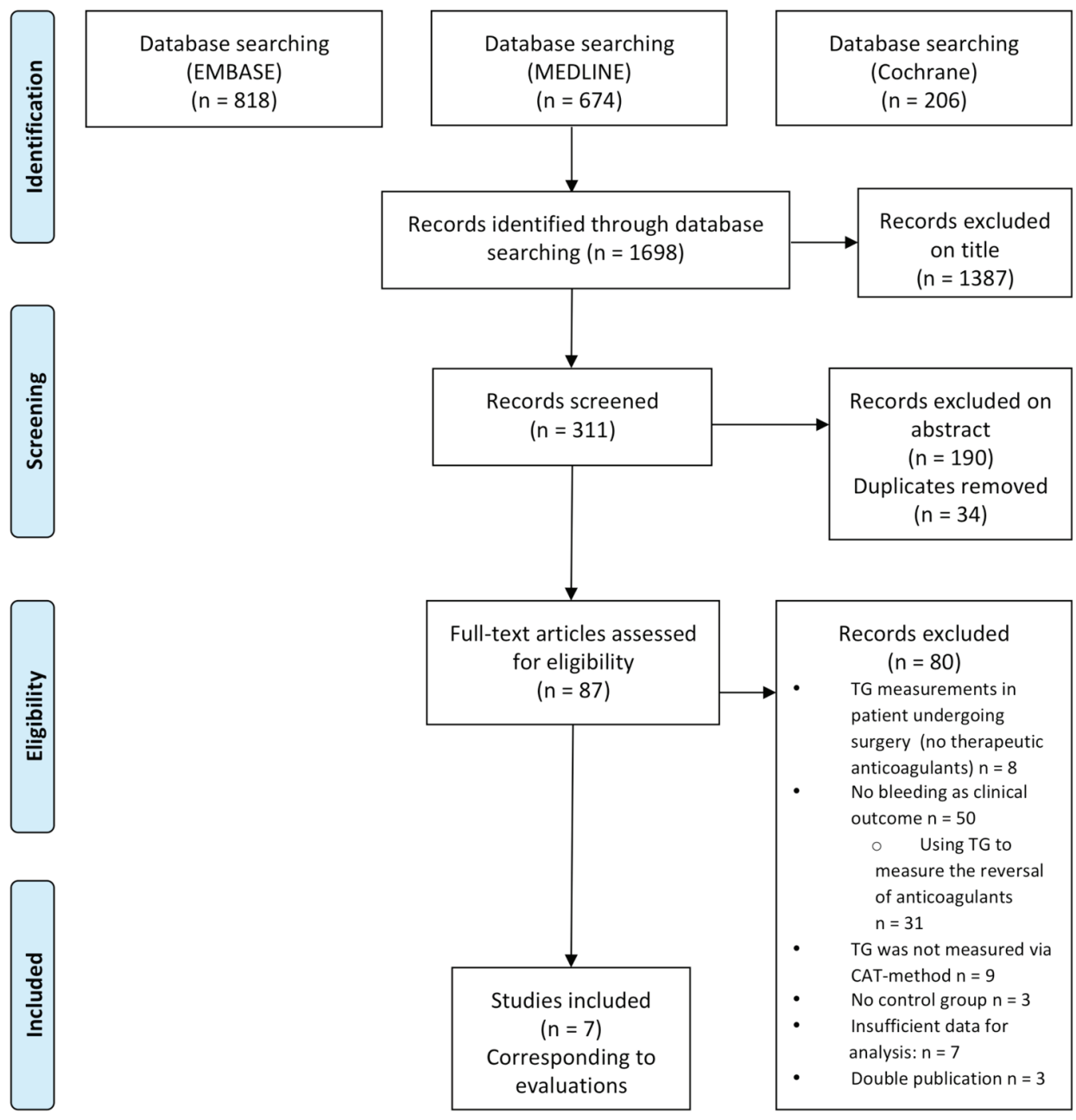

Figure 1. Flow diagram of literature review.

$\mathrm{TG}=$ thrombin generation; $\mathrm{CAT}=$ calibrated automated thrombinography

\section{General aspects and results of the included studies}

The studies selected were mostly prospective studies. There are some similarities between the studies concerning the choice of anticoagulants, the target range for the INR and the investigated method of TG. There was heterogeneity in patient selection, duration of follow-up and also in registration of outcome parameters. A general overview of the included studies is given in table 1. The CAT-TG values measured in bleeding and non-bleeding patients using VKAs are displayed in table 2. The quality of the included studies was rated according the Newcastle-Ottawa Quality assessment scale table (table $3 a$ and 3b). 


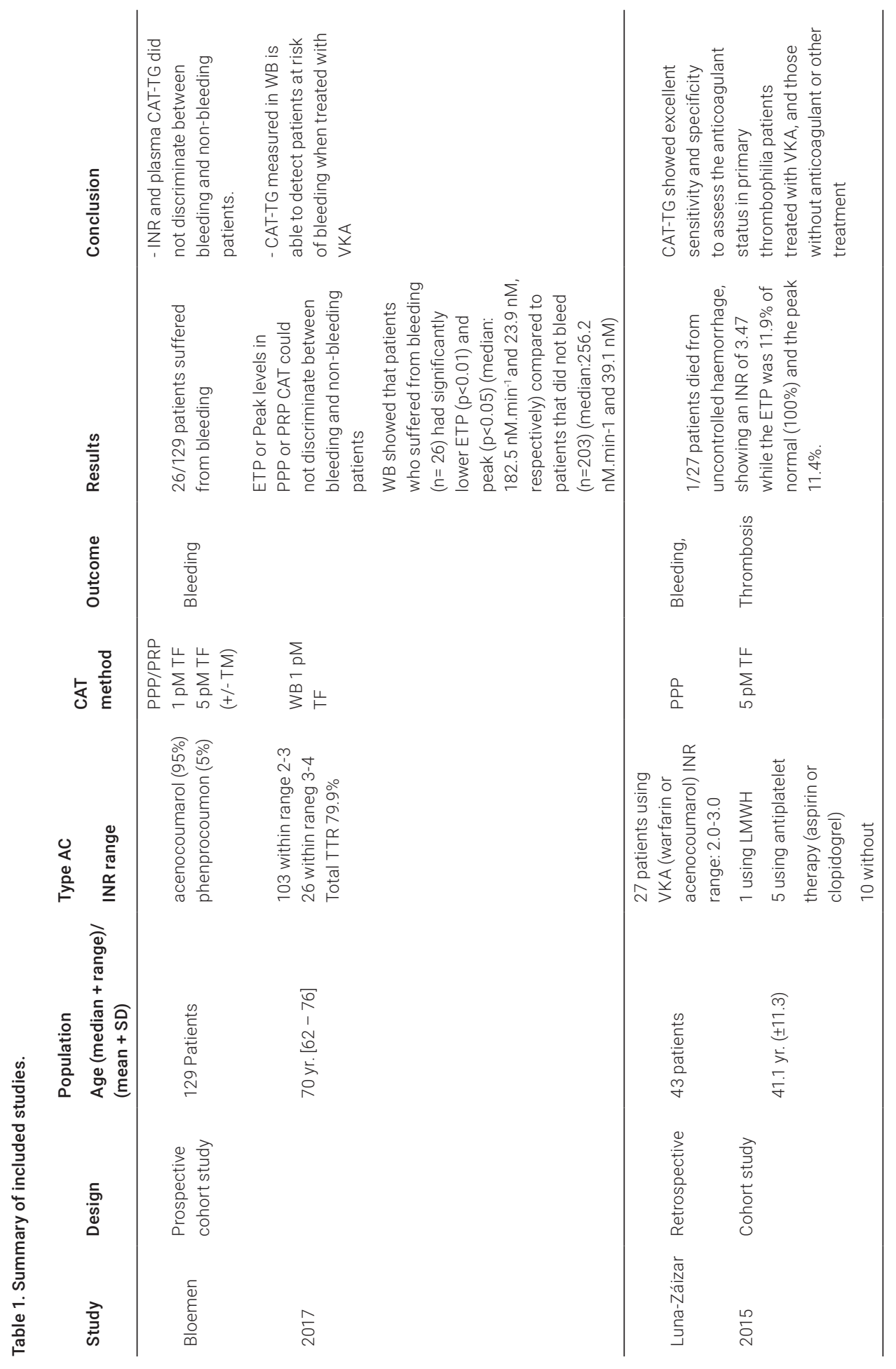




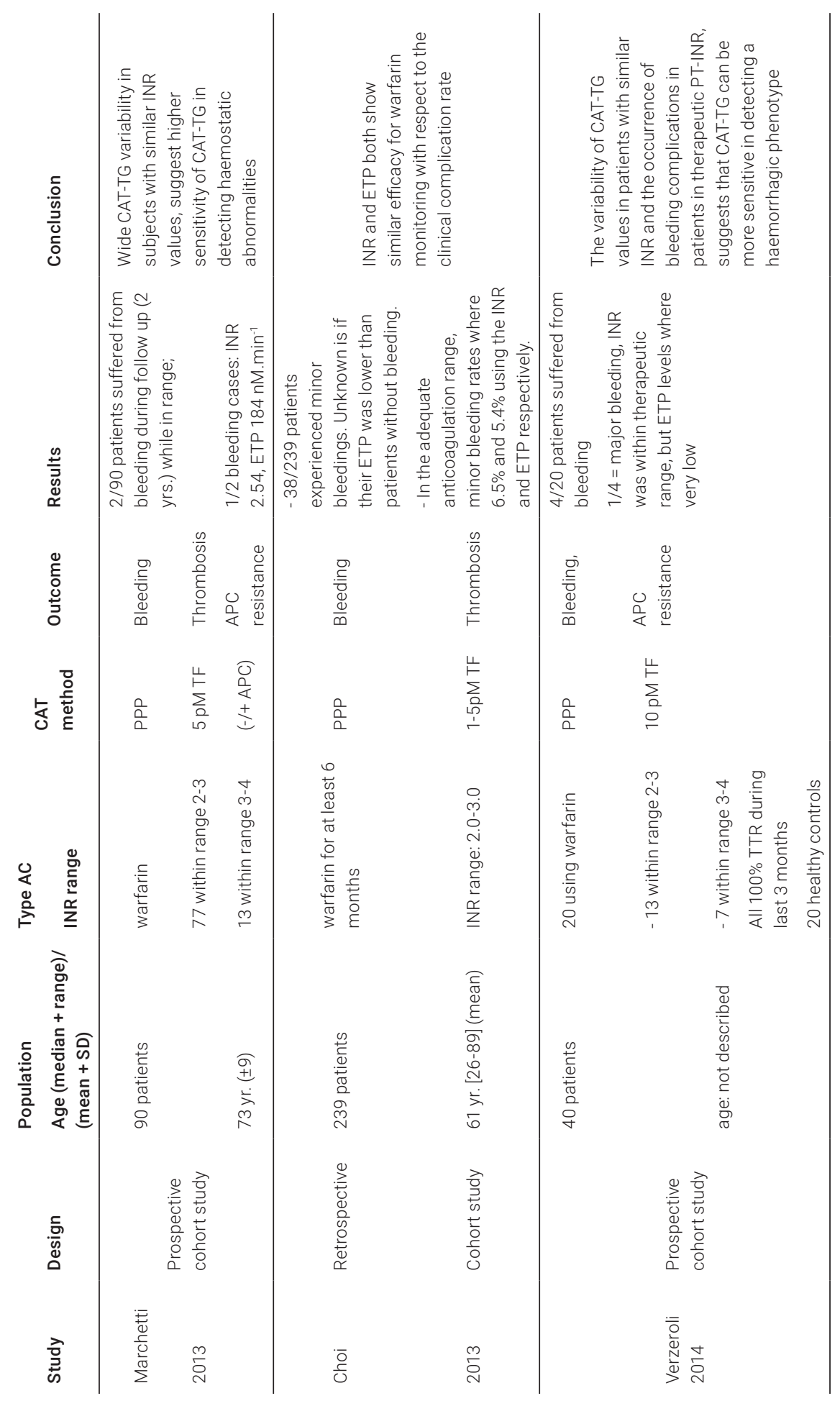




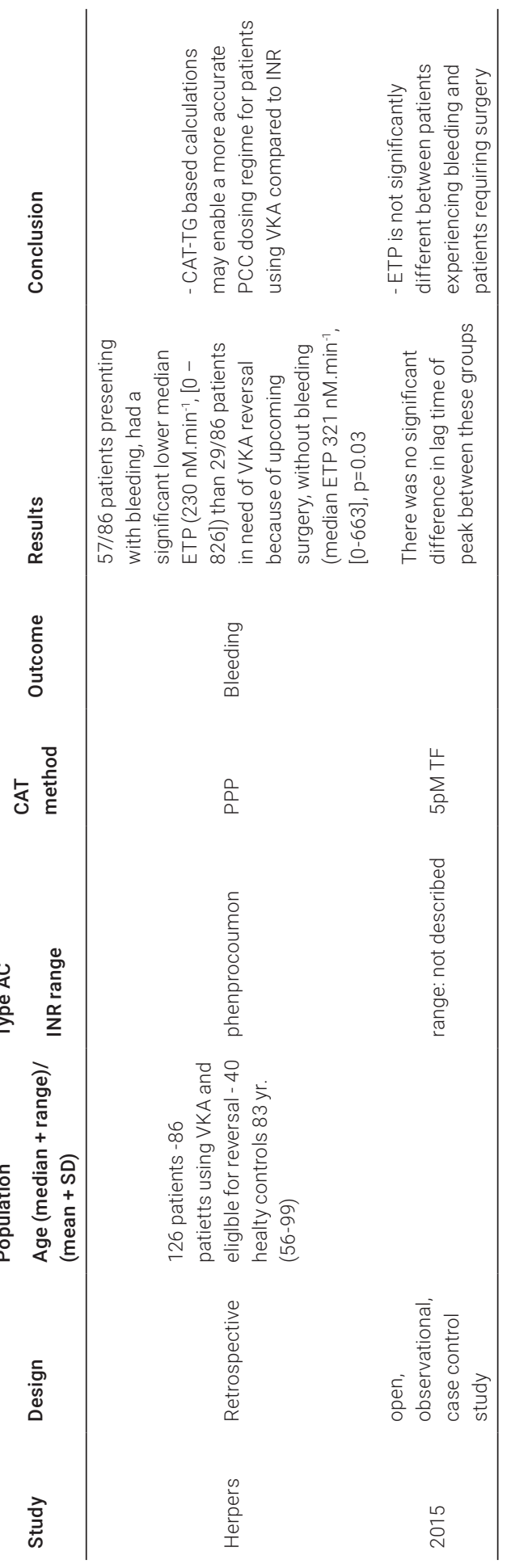




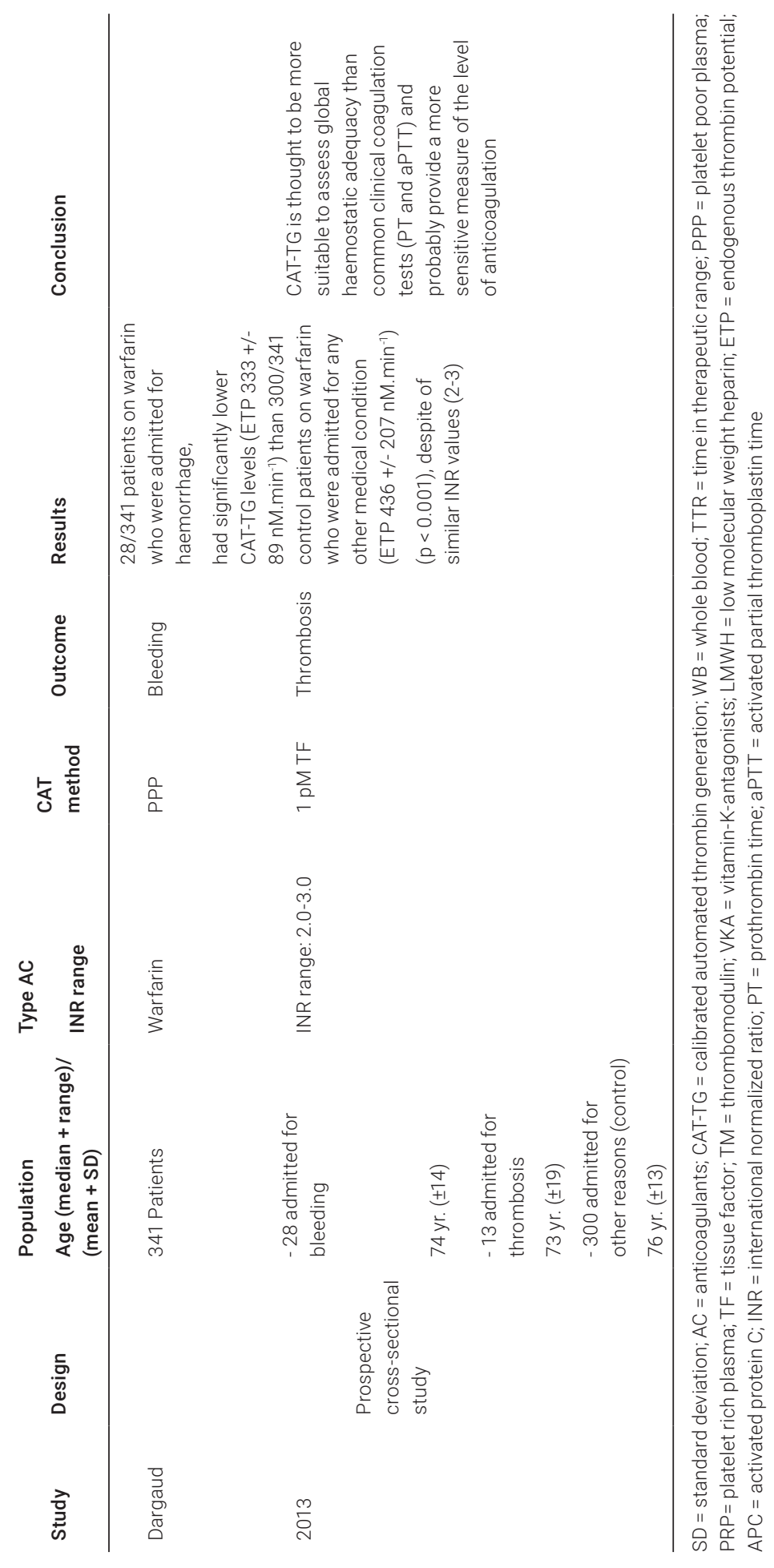




\section{Cohort studies:}

Bloemen et al. studied whether CAT-TG either in plasma or in whole blood could be used to predict bleeding episodes in patients using VKAs. ${ }^{55}$ The authors included 129 patients who used VKAs for at least 3 months with an average age of 68 years. Of this population $21.7 \%$ were female and $95.4 \%$ of the patients were treated with acenocoumarol. The average time in therapeutic range (TTR) was 79.9\%. Of the included patients, 103 were classified in the lower INR range (2.0-3.0) and 26 patients in the higher range (2.5-4.0). Patient characteristics were well defined and there was a follow-up of 15.5 months. As main outcome clinically relevant bleeding episodes were scored during follow-up according to the criteria defined by the Dutch Federation of Anticoagulation Clinics (FNT). Clinically relevant bleeding episodes occurred in 26 (20.2\%) patients. In total 44 bleeding episodes were registered, which is due to the fact that some patients had multiple episodes. CAT-TG measured in PPP with 5pM TF showed lower ETP levels in patients who had experienced bleeding compared to patients without bleeding, however this difference was not significant. Also other CAT-TG parameters like peak height could not discriminate between bleeding and non-bleeding patients (P-value $=0.18)$ nor could INR (P-value $=0.87)$, hematocrit $(P$-value $=0.23)$, hemoglobin (P-value = 0.11), or fibrinogen level (P-value $=0.54)$. In contrast, an increased HAS-BLED score was significantly associated with bleeding (P-value <0.05). Interestingly, CAT-TG stimulated with $1 \mathrm{pM}$ of TF in whole blood yielded significantly lower ETP $(p<0.01)$ and peak height levels $(P<0.05)$ in patients that suffered from bleeding, compared to patients who did not bleed.

Luna-Záizar et al. explored the usefulness of CAT-TG to assess the anticoagulation status compared with the INR in patients with primary thrombophilia. ${ }^{56}$ Fifty patients, who were diagnosed with inherited thrombophilia and had experienced at least one thrombotic event, were included. Whether the selection was consecutive is not described, neither are the criteria for exclusion. Definitions of the outcome "bleeding" (major or minor) were not further explained. Complete results were obtained from 43 patients (13 males (30\%)/ 30 females (70\%), mean age $41.1+/$ - 11.3 years). Twenty-seven of these patients were treated with VKAs and one patient was treated with low molecular weight heparin (LMWH). Fifteen patients were either without anticoagulation medication or used antiplatelet therapy only. CAT-TG was initiated with 5 pM TF in PPP. During the study only one bleeding event occurred. This patient on VKA treatment suffered from fatal haemorrhage. While the INR was 3.47, ETP and peak levels were lower than CAT-TG values of patients using VKAs (2.0-3.0) who did not bleed. Furthermore the study showed significant lower ETP and peak values for patients 
who used VKAs or LMWHs compared to patients who were untreated $(P<0.0001)$. Lag time was also discriminative between these groups but displayed great interindividual variability in the group of patients under optimal anticoagulation with VKAs (INR 2.0-3.0). An inverse nonlinear relation was found between ETP values $\left(R^{2}=0.649\right)$, peak $\left(R^{2}=0.633\right)$ and the velocity index $\left(R^{2}=0.532\right)$ vs. INR values in patients treated with VKAs. As expected a positive linear correlation between lag time and INR was found $\left(R^{2}=0.338\right)$.

Marchetti et al. performed a prospective cohort study to investigate the characteristics of CAT-TG in patients with atrial fibrillation (AF) on permanent oral anticoagulation therapy with warfarin ${ }^{57}$. They included 90 patients (56 males/34 females; aged $73+/-9$ years.) of whom 77 patients had a target INR within range 2.0-3.0, and 13 patients within 3.0-4.0. Other patient characteristics such as concomitant use of anti-platelet therapy were not presented. Outcomes of interest were bleeding and thrombosis, but predefined criteria for bleeding (minor or major) or thrombosis were not described in detail. Patients were followed-up for an average time of 2 years. During follow-up 2 bleedings occurred: one patient suffered a gastric bleeding and another patient experienced cerebral haemorrhage, both of them being within the INR target range. One of these patients with an INR of 2.54 did have a low ETP value. CAT-TG was performed using 5 pM TF. Besides the ETP also other parameters were evaluated: Peak height, lag time and time-to-peak. A significantly decreased ETP (435 +/- 190 vs. 1229 +/- 114 nM.min-1) and Peak (109 +/- 51 vs. 256 +/- 44 nM) were observed in patients with stable INRs in their appropriate range, compared with healthy controls ( $P$ $<0.01)$. In addition, lag time and time-to-peak were significantly prolonged in the patient group: (4.1+/- 1.5 vs. 2.0 +/- $0.3 \mathrm{~min})$ and ( $6.2+/-1.7$ vs. $4.6+/-0.6 \mathrm{~min})$, respectively. A moderate correlation between INR and all CAT-TG parameters $\left(R^{2}=0.6\right)$ was found, but on the other hand patients with similar INRs showed a large variability in TG levels, particularly the patients within target INR range 2.0-3.0.

Choi et al. compared the monitoring performance of ETP with INR. ${ }^{58}$ In this retrospective cohort study, 239 patients (129 males/110 females; mean age 61 years, range: 26-89 years) were included using warfarin for at least 6 weeks for several indications (prosthetic heart valves, AF, ischemic heart failure or DVT). The INR ranged from 1.0 to 4.26 . Exclusion criteria were $<19$ years old, the use of other anticoagulant treatment than warfarin or the use of antiplatelet agents. Outcome was bleeding and thrombosis, obtained from medical records. TG was performed using the CAT-method. The amount of TF and PL was not 
clearly mentioned, but a reference was made to the article of Hemker et al. in which $5 \mathrm{pM}$ TF and $4 \mu \mathrm{M}$ PL were used. ${ }^{59} \mathrm{~A}$ significant inverse correlation between ETP and INR was established $(r=-0.769, P<0.001)$. With a regression equation the authors calculated a therapeutic range for the ETP $\left(290.1\right.$ - $\left.494.6 \mathrm{nM} \cdot \mathrm{min}^{-1}\right)$, which would correspond to the INR range from 2.0-3.0. Subsequently, the patients were divided into 3 groups (under-, adequately- and over-anticoagulated) according to their anticoagulation status judged by the INR or ETP. Whether the ETP is measured per individual or calculated based on the formula mentioned above remains uncertain. To assess the monitoring performance of the INR and ETP they compared their anticoagulation status to clinical complications rates. When the division to the adequate anticoagulation group was based on INR or ETP, bleeding rates where $6.3 \%$ and $5.4 \%$ respectively. Although the use of ETP as target parameter yielded a lower bleeding rate, the difference was not statistically significant. During the study only minor bleedings occurred in 38 patients. Individual ETP values were not reported in the article.

Verzeroli et al. investigated the correlation between CAT-TG and INR in patients treated with warfarin and whether CAT-TG could be useful to identify subjects at a higher risk of bleeding. ${ }^{60}$ The study included 20 patients using warfarin (13 for AF, 7 for cardiac valve prosthesis) who had been in their appropriate target INR ranges for $100 \%$ over the last 3 months; 20 healthy subjects were studied as controls. Patient characteristics were not described, nor were INR target ranges specified. Patients were followed for 1 year. CAT-TG when activated with 10 pM TF, showed a significantly lower TG potential in the patients using warfarin compared to healthy controls; a significant correlation between the INR levels and all TG parameters $(P<0.01)$ was mentioned but the actual data on correlations were not given. During follow-up, bleeding complications were registered in 4 patients, of which 1 major, while all patients were in their respective target INR ranges. All 4 patients had a very low TG potential, but exact levels of CAT-TG parameters are not described. The diagnostic criteria of "bleeding" were not further defined. 
Table 2. CAT-TG values compared in bleeding and non-bleeding patients using VKA.

\begin{tabular}{|c|c|c|c|c|c|c|c|c|c|}
\hline \multirow[b]{2}{*}{ Author: } & \multicolumn{4}{|c|}{ Bleeding } & \multicolumn{5}{|c|}{ Non-bleeding } \\
\hline & CAT-TG & $\mathrm{N}$ & & $\begin{array}{l}\text { Median/ } \\
\text { Mean }\end{array}$ & $\begin{array}{l}I Q R / \\
S D\end{array}$ & $N$ & $\begin{array}{l}\text { Median/ } \\
\text { Mean }\end{array}$ & $\begin{array}{l}I Q R / \\
S D\end{array}$ & $p$-value \\
\hline \multirow[t]{8}{*}{ Bloemen } & WB & 26 & $\operatorname{ETP}\left(\mathrm{nM} \cdot \mathrm{min}^{-1}\right)$ & 182.5 & $157-285$ & 101 & 256.2 & $195-344$ & $<0.01^{\star \star}$ \\
\hline & & & Peak (nM) & 23.9 & $20-42$ & & 39.1 & $25-53$ & $<0.05^{\star}$ \\
\hline & & & TtPeak (min) & 12,2 & $10-18$ & & 12.2 & $10-15$ & 0.427 \\
\hline & & & Lagtime (min) & 7.1 & $5-8$ & & 6.5 & $5-8$ & 0.545 \\
\hline & PPP & 26 & $\operatorname{ETP}\left(\mathrm{nM} \cdot \mathrm{min}^{-1}\right)$ & 367 & $298-501$ & 101 & 438 & $344-541$ & 0.09 \\
\hline & & & Peak (nM) & 76 & $59-99$ & & 86.6 & $63-114$ & 0.181 \\
\hline & & & TtPeak (min) & 7.8 & $6-10$ & & 8.3 & $7-10$ & 0.897 \\
\hline & & & Lagtime (min) & 5.1 & $4-7$ & & 5.5 & $4-7$ & 0.805 \\
\hline \multirow[t]{3}{*}{ Luna-Záizar } & PPP & 1 & $\begin{array}{l}\text { ETP } \\
\% \text { of normal } \\
\text { (normal }=100 \% \text { ) }\end{array}$ & $11.9 \%$ & & 26 & $31.2 \%$ & \pm 12 & $\mathrm{n} / \mathrm{a}$ \\
\hline & & & $\begin{array}{l}\text { Peak } \\
\% \text { of normal) } \\
\text { (normal = } 100 \% \text { ) }\end{array}$ & $11.4 \%$ & & & $32 \%$ & \pm 10 & $\mathrm{n} / \mathrm{a}$ \\
\hline & & & Lagtime (min) & $\mathrm{n} / \mathrm{a}$ & & & 6 & \pm 2 & $\mathrm{n} / \mathrm{a}$ \\
\hline \multirow[t]{4}{*}{ Marchetti } & PPP & 2 & $\operatorname{ETP}\left(\mathrm{nM} \cdot \mathrm{min}^{-1}\right)$ & $184^{+}$ & & 90 & 435 & \pm 190 & $\mathrm{n} / \mathrm{a}$ \\
\hline & & & Peak (nM) & 76 & & & 109 & \pm 51 & $\mathrm{n} / \mathrm{a}$ \\
\hline & & & TtPeak (min) & $\mathrm{n} / \mathrm{a}$ & & & 6 & \pm 2 & $\mathrm{n} / \mathrm{a}$ \\
\hline & & & Lagtime (min) & $\mathrm{n} / \mathrm{a}$ & & & 4 & \pm 2 & $\mathrm{n} / \mathrm{a}$ \\
\hline Choi & PPP & 38 & & $\mathrm{n} / \mathrm{a}$ & & 239 & $\mathrm{n} / \mathrm{a}$ & & $\mathrm{n} / \mathrm{a}$ \\
\hline Verzeroli & PPP & 4 & & $\mathrm{n} / \mathrm{a}$ & & 20 & $\mathrm{n} / \mathrm{a}$ & & $\mathrm{n} / \mathrm{a}$ \\
\hline \multirow[t]{3}{*}{ Herpers } & PPP & 57 & $\operatorname{ETP}\left(\mathrm{nM} \cdot \mathrm{min}^{-1}\right)$ & 230 & $(0-826)$ & 29 & 321 & $(0-663)$ & $0.03^{\star}$ \\
\hline & & & Peak (nM) & 27 & $(0-208)$ & & 42 & $(0-145)$ & $>0.05$ \\
\hline & & & Lagtime (min) & 12 & $(3->60)$ & & 8 & $(4->60)$ & $>0.05$ \\
\hline Dargaud & PPP & 28 & $\operatorname{ETP}\left(\mathrm{nM} \cdot \mathrm{min}^{-1}\right)$ & 333 & \pm 89 & 300 & 436 & \pm 207 & $<0.001^{\star \star}$ \\
\hline
\end{tabular}

Mean values with SD are in bold, the other values are medians with interquartile ranges. CAT-TG = calibrated automated thrombin generation; $\mathrm{N}=$ number; $\mathrm{IQR}$ = interquartile range; $\mathrm{SD}=$ standard deviation; $\mathrm{WB}=$ whole blood; PPP = platelet poor plasma; $\mathrm{ETP}=$ endogenous thrombin potential; $T$ tpeak = time-to-peak; $\mathrm{n} / \mathrm{a}=$ non applicable

$\star$ Significant difference $p<0.05 ; \star$ significant difference $p<0.01$

† Only CAT-TG values from one patient were reported, although two patients suffered from bleeding 


\section{Case control studies:}

Herpers et al. compared the use of the INR versus CAT-TG to guide VKA reversal ${ }^{35}$. In an open, observational study they studied 86 patients treated with phenprocoumon, who where in need of VKA reversal. The median age was 83 years [56-99 years], 53\% was female and the indication for VKAs was mostly cardiac arrhythmia (84\%). They compared 29 patients who needed prophylactic VKA reversal because of upcoming surgery to 57 patients who needed VKA reversal because of haemorrhage. Coagulation reactions during CAT-TG were initiated with 5 pm TF. A significantly lower ETP was described in bleeding patients compared to non-bleeding patients. Lag time and peak did not differ significantly $(P>0.05)$ between these groups. However, in the discussion and conclusion the authors state that no significant difference was found in any CAT parameter (including the ETP).

Dargaud et al. evaluated the hypothesis that the INR might underestimate the level of anticoagulation in patients with a lower Factor IX level in contrast to CAT-TG, suggesting CAT-TG to be a more accurate test. ${ }^{61}$ In this study, 341 patients on warfarin with a stable INR between 2.0-3.0 were included at admission to the emergency department. Twenty-eight of these patients (18 males/10 females, aged $74+/$ - 14 years) were admitted for haemorrhage. Thirteen patients were admitted for thrombosis and were not taken into account in this review. Three hundred patients were admitted for other medical reasons (151 males/149 females, aged $76+/-13$ years). Of the 28 patients who were admitted for haemorrhage, 22 had major bleeding (7 spontaneous intracranial haemorrhage, 3 trauma related intracranial haemorrhage, 12 with gastrointestinal tract bleeding), 5 patients exhibited muscle haematomas; severe epistaxis or gum bleeding and 1 patient had urinary tract bleeding. All patients were reviewed for potential underlying disorders that might explain their bleeding episodes, but no significant abnormalities were detected. The criteria for major bleeding were not explained in further detail. All CAT-TG experiments were activated with 1 pM TF. Patients on warfarin who were admitted for haemorrhage $(n=28)$, had significantly lower TG levels than patients on warfarin that were admitted for any other medical condition, while having similar INRs in target range. No significant correlation between the INR levels in range 2.0-3.0, and ETP values $(r=-0.05,95 \% \mathrm{Cl}=-0.168$ to $0.059 ; \mathrm{P}=0.361)$ was found. Mean TG levels of all admitted patients on warfarin with INRs in target range 2-3 $(n=341)$ were significantly lower than TG levels of 100 healthy controls (ETP $428+/-200 \mathrm{nM} \cdot \mathrm{min}^{-1}$ vs. ETP $\left.1380+/-214 \mathrm{nM} \cdot \mathrm{min}^{-1}\right)$. 


\section{Thrombin Generation methods in the included studies}

All studies measured TG by using the CAT-method, although this method was not always adequately described. Bloemen, Luna-Záizar, Choi, Herpers and Dargaud both used the CAT-method according to Hemker et al. ${ }^{59}$ The assays were performed on a Fluorscan Ascent ${ }^{\circledR}$ fluorometer (thermolabsystem OY, Helsinki, Finland). The software program, Thrombinoscope ${ }^{\circledR}$ (Thrombinoscope BV), enabled the calculation of thrombin activity. Bloemen used $1 \mathrm{pM}$ as well as $5 \mathrm{pM}$ tissue factor (TF) to initiate coagulation in PPP. Dargaud used 1 pM TF. Luna-Záizar and Herpers both used 5 pM TF, while Choi does not mention the used amount of TF. Bloemen et al. measured CAT-TG also in whole blood, using 1 pm TF, according the specifications of Ninivaggi et al. ${ }^{62}$ Marchetti and Verzeroli et al. did not describe the CAT-method in detail. They did report the amount of TF used, which was 5 pM and $10 \mathrm{pM}$ respectively.

All studies used the same concentration of phospholipids $(4 \mu \mathrm{M})$. Two studies (Dargaud and Bloemen) reported the specifications of the type of phospholipids used; both used phospholipids obtained from Avanti Polar Lipids (Alabaster, AL, USA).

\section{Table 3a. Newcastle Ottawa Quality Assessment Scale - Cohort Studies.}
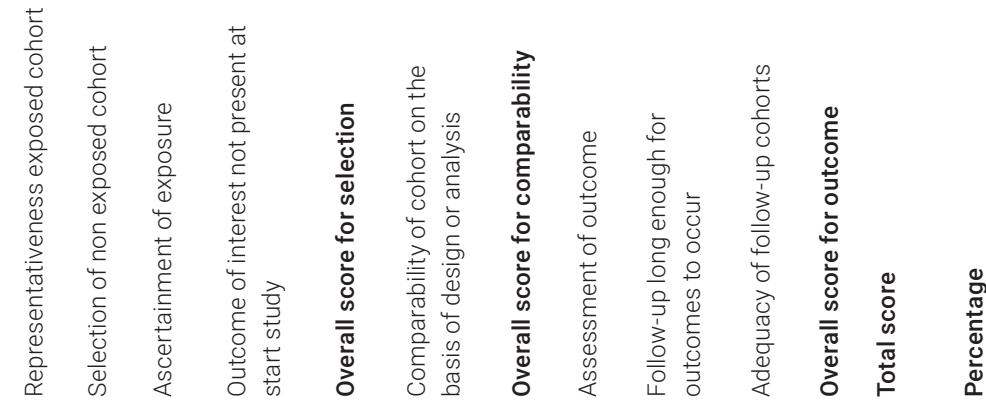

\begin{tabular}{|c|c|c|c|c|c|c|c|c|c|c|c|c|c|}
\hline $\begin{array}{l}\text { Bloemen } \\
2017\end{array}$ & $\star$ & $\star$ & $\star$ & - & 3 & $\star \star$ & 2 & $\star$ & $\star$ & - & 2 & $7 / 9$ & $77.7 \%$ \\
\hline $\begin{array}{l}\text { Luna-Záizar } \\
2015\end{array}$ & $\star$ & $\star$ & $\star$ & - & 3 & - & 0 & $\star$ & - & - & 1 & $4 / 9$ & $44.4 \%$ \\
\hline $\begin{array}{l}\text { Verzeroli } \\
2014\end{array}$ & - & $\star$ & $\star$ & - & 2 & $\star$ & 1 & - & $\star$ & - & 1 & $4 / 9$ & $44.4 \%$ \\
\hline $\begin{array}{l}\text { Choi } \\
2013\end{array}$ & $\star$ & $\star$ & $\star$ & - & 3 & $\star$ & 1 & $\star$ & - & - & 1 & $5 / 9$ & $55.5 \%$ \\
\hline $\begin{array}{l}\text { Marchetti } \\
2013\end{array}$ & - & $\star$ & $\star$ & - & 2 & $\star$ & 1 & - & $\star$ & - & 1 & $4 / 9$ & $44.4 \%$ \\
\hline
\end{tabular}


Table 3b. Newcastle Ottawa Quality Assessment Scale - Case Control Studies.

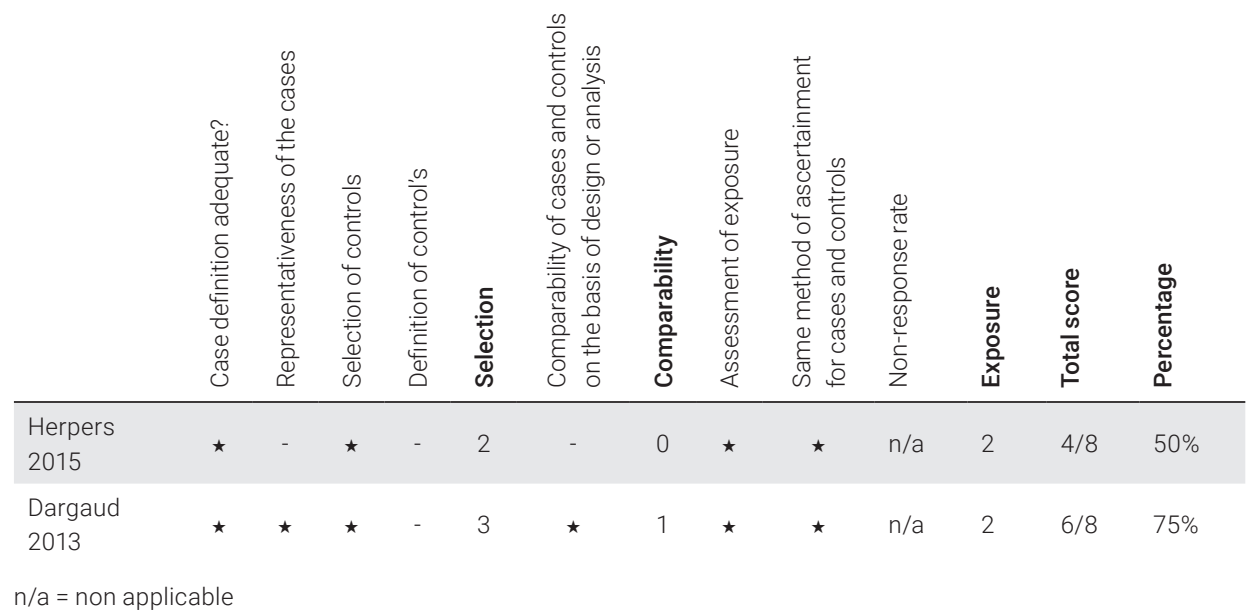

\section{DISCUSSION}

Haemorrhagic complications remain the most important concern during management of anticoagulation therapy. Insight in the (individual) bleeding risk of patients would likely affect clinical decision-making. However, sensitive instruments to identify patients at increased risk of bleeding, who would benefit from more careful management, are lacking. CAT-TG measures the total amount of thrombin formed over time, and offers a more global assessment of coagulation because it is influenced by all clotting factors involved in the cascade. ${ }^{63}$ Several studies have investigated the role TG as a new tool to assess coagulation.13,15,64,65 This review was performed in order to assess the currently available evidence for the predictive ability of CAT-TG in relation to bleeding, not related to surgery, in patients on anticoagulant treatment.

We retrieved 1698 papers when searching the key words "thrombin generation", "anticoagulant therapy" and "bleeding". Interestingly, most studies correlated CAT-TG to the INR, based on the known association between INR and risk of bleeding, to indirectly link bleeding risk to CAT-TG values. In our search we also identified 31 papers exploring the value of TG for the assessment of the reversal of different anticoagulants, including the direct oral anticoagulant drugs. TG levels were used as reference point to determine whether coagulation was restored after adding three- or four-factor prothrombin complex concentrate (PCC), fresh frozen plasma (FFP) or factor VIII inhibitor bypass activity (FEIBA).13,17-43 
Remarkably few studies investigated the direct relation between CAT-TG values and clinical bleeding in stable anticoagulated patients, and could therefore be included in this systematic review. Of these 7 studies only 2 studies were of good methodological quality. The quality of the others studies was considered as moderate. The data available from the studies was unfortunately insufficient to perform a meta-analysis. There are multiple techniques to measure TG. In this review we chose to solely evaluate TG measured by the CAT-method. The difference between methods, as well in protocol as in reagents, makes the TG data between techniques hard to compare. Since TG measured with CAT was previously found to be correlated to a hypocoagulable, as well as a hypercoagulable state in patients, we decided to investigate TG measures with CAT only, although this would limited our scope. 52,66,67 Moreover, to fairly compare outcomes between different studies, a standardized protocol of the CAT method is important. Although the protocols of the included study were largely identical, two studies used different amounts of TF in CAT, which made comparison between outcomes of studies difficult. The use of lower amounts of TF e.g. 1 pM, will enhance the sensitivity of the assay, but could reduce the reproducibility. Choosing a high concentration of TF (e.g.) $10 \mathrm{pM}$, the contribution of the intrinsic pathway to coagulation will become negligible.

This review stressed the need for an unambiguous study protocol for CAT-TG. Some of the included studies were limited because of a small sample size. Additionally, in all included cohort studies the number of patients that suffered from bleeding during the follow up was lower compared to the patients that did not bleed. Consequently, the number of bleeding events may have been too low to reach solid conclusions about the predictive value of CAT-TG for bleeding. The study by Dargaud found significantly lower mean ETP levels in patients experiencing warfarin-related haemorrhage compared to patients with similar INR values in the desired range, who did not experience bleeding. This study had sufficient power and enough clinical bleedings (8.2\%) to point out significant differences in ETP levels between bleeding and non-bleeding patients. However, the design of this study may not be suitable to answer the question whether low CAT-TG values are predictive for bleeding. In this cross-sectional study, the patients suffered from warfarin-related haemorrhage at the time of inclusion and blood withdrawal for the study assessment. Therefore the ETP levels most likely represent a more acute haemostatic condition, involving bleeding-associated consumption of coagulation proteins. Herpers et al. investigated whether patients in need of VKA reversal because of bleeding had lower ETP than patients in need of VKA reversal who did not bleed. This study was considered of moderate methodological quality, even more so because in the discussion and the conclusions of the paper contradictory interpretations of the findings were presented. 
In addition, the difference in design makes it difficult to compare the results of previous studies with the other included studies, which were cohort studies. In the cohort studies, the ETP levels are measured at inclusion while patients are in a stable, non-bleeding state, and bleedings are registered during follow up. The latter design is more informative on the predicting ability of CAT-TG. Unfortunately, out of the five included prospective cohort studies only two studies were of acceptable methodological quality. The studies of Marchetti and Vezeroli both suggest that the differences in ETP values found in patients with similar INRs, indicate that CAT-TG is more sensitive in detecting haemostatic abnormalities than INR. However, data were derived from conference abstracts only, therefore information was incomplete. Luna-Záizar et al. examined the use of CAT-TG in patients using VKAs to assess the anticoagulation status compared to the INR, which corresponds to our research question. The quality of the study was rated as moderate. The sample size was small and only one (major) bleeding event was described during follow up. This patient had lower ETP values compared to other patients with similar INRs but did not bleed. The authors state that this clinical event suggests that the ETP can predict an increased haemorrhagic risk, making CAT-TG a better monitoring parameter than the INR. However, these conclusions are based on a single event rendering the outcome highly questionable. The research question of Choi et al. was also in accordance with ours. The sample size of this study was sufficient and patients as well as controls were drawn out of the community. At the same time the follow up was poorly described. Moreover, the individual ETP values of patients could not be extracted from the data, making it impossible to compare ETP between bleeding and non-bleeding VKA patients. Patients were divided into groups based on their INR or ETP value. The calculation made to develop a therapeutic range for ETP, which should compare to the INR range 2.0-3.0, was partly based upon the INR. Although the article shows an inverse correlation between INR and ETP, which is linear between the INR range 2.0-3.0, it can not be assumed that this correlation will remain linear over a larger range. ${ }^{15,68}$ Therefore ETP values comparable with INRs outside the range 2.0-3.0 cannot be justified. Overall the results of this study were not suitable to answer our research question mainly because of the design despite the general moderate quality. The study by Bloemen et al. was considered of fairly good methodological quality. The design was suitable to answer our research question and gave a detailed description of inclusion criteria, patient characteristics and the specific execution of the CAT-TG test. Although lower average ETP levels were seen in the patients who experienced bleeding compared to patients who did not, no significant difference was observed in the platelet poor plasma CAT-TG. It could be argued that this study was underpowered, and therefore not able to answer the hypothesis if CAT-TG can predict bleeding in patients. When a larger number of patients would have been 
investigated a significant difference might be found, but a substantial overlap in ETP-values between groups will remain, rendering CAT-TG in PPP probably not discriminative between bleeding and non-bleeding patients. One of the limitations of this study was a lack of correction for several confounding factors that are associated with a higher bleeding risk, e.g. diabetes mellitus, a reduced kidney function or a positive bleeding history. Additionally, the occurrence of bleeding complications during follow-up was higher than expected (20.2\%) and most bleedings were clinically relevant minor bleeds. Previous studies have shown that minor bleedings during anticoagulant therapy can be predictive for subsequent major bleedings, although the underlying causal mechanism for this is still to be elucidated. ${ }^{69,70}$ Some evidence indicates that minor bleedings are a marker for fixed and currently unknown risk factors for major bleeding events. In the study by Bloemen et al. it was shown that CAT-TG measured in whole blood was able to distinguish patients at risk of bleeding. One explanation for these findings could be the concurrent effect of platelets and red cells in the whole blood thrombin generation assay. Since a previous study from our laboratory did not detect any significant influence of platelets on bleeding risk in anticoagulated patients, by demonstrating in platelet rich plasma (PRP) that platelet function tests and von Willebrand factor levels did not differ between bleeding and non-bleeding patients on VKAs ${ }^{71}$ a contribution from red blood cells or leukocytes is likely. Although the findings of Bloemen et al. in whole blood are promising, whole blood TG is only recently developed, and the present study provides the first implementation in patients on VKAs. More studies are needed to see if these results can be confirmed.

To genuinely evaluate the predictive ability of CAT-TG in relation to bleeding events it is required to investigate the sensitivity, specificity, positive and negative predictive value of the assay, in order to calculate an odds ratio or construct an receiver operation characteristic (ROC) curve. Unfortunately, this was not possible due to the absence of predefined cut-off values of CAT-TG parameters and because most articles compared mean or median CAT-TG values between patients who suffered from bleeding and patients who did not. Individual CAT-TG values of bleeding and non-bleeding patients were not reported.

Unfortunately, no papers were found investigating the value of CAT-TG to predict bleeding in patients using the direct oral anticoagulants. This could be explained by different reasons. First, measuring direct lla inhibitors with TG shows a paradoxical increase of the peak and ETP and is therefore currently not reliable enough. ${ }^{72,73}$ Another explanation could be that measurements of direct Xa inhibitors, although possible with CAT-TG, have not yet been correlated to clinical bleeding complications in humans. 


\section{CONCLUSION}

CAT-TG is frequently used to assess the effects of anticoagulation in different research settings. Clinical studies are mostly performed in patients treated with VKAs, showing decreased TG values in patients compared with healthy controls. 14,59,61,74 The studies of Bloemen and Dargaud et al., which were found to be of the highest methodological quality according this review, both found evidence supporting an association between low CAT-TG values and bleeding in patients using VKAs. Other reviewed studies agreed with this reasoning, but did not provide enough data to validate this hypothesis. Unfortunately, studies investigating the direct association between decreased CAT-TG values and actual haemorrhagic events are scarce, therefore the clinical consequences of low CAT-TG values remain to be investigated. To further evaluate whether low CAT-TG values can identify patients with a higher bleeding risk, new studies are needed.

While application in VKA treatment is an interesting avenue to pursue, it may be even more important look for associations between CAT-TG activity and bleeding outcomes in patients using DOACs. The use of fixed doses of DOACs, based solely on patient characteristics, causes a wide variability in their anticoagulant responses, which makes testing with overall assays like CAT-TG potentially interesting.

\section{DECLARATION OF INTEREST}

Hugo ten Cate is consultant to Stago, manufacturer of the CAT; he is also chair of the board of the Dutch Federation of Anticoagulation Clinics. All other authors declared to have no conflicts of interest. Suzanne Zwaveling and Saartje Bloemen are linked to and Bas de Laat is employed at Synapse Research Institute, which is part of Stago. 


\section{REFERENCES}

1. Ansell J, Hirsh J, Hylek E, Jacobson A, Crowther M, Palareti G. Pharmacology and management of the vitamin K antagonists: American College of Chest Physicians Evidence-Based Clinical Practice Guidelines (8th Edition). Chest. 2008;133(6 Suppl):160s-198s.

2. Veeger NJ, Piersma-Wichers M, Tijssen JG, Hillege HL, van der Meer J. Individual time within target range in patients treated with vitamin $\mathrm{K}$ antagonists: main determinant of quality of anticoagulation and predictor of clinical outcome. A retrospective study of 2300 consecutive patients with venous thromboembolism. $\mathrm{Br} \mathrm{J}$ Haematol. 2005;128(4):513-519.

3. Palareti G, Leali N, Coccheri S, et al. Bleeding complications of oral anticoagulant treatment: an inception-cohort, prospective collaborative study (ISCOAT). Italian Study on Complications of Oral Anticoagulant Therapy. Lancet. 1996;348(9025):423-428.

4. Jackson SL, Peterson GM, Vial JH, Daud R, Ang SY. Outcomes in the management of atrial fibrillation: clinical trial results can apply in practice. Intern Med J. 2001;31(6):329-336.

5. Abdelhafiz AH, Wheeldon NM. Results of an open-label, prospective study of anticoagulant therapy for atrial fibrillation in an outpatient anticoagulation clinic. Clin Ther. 2004;26(9):1470-1478.

6. Connolly SJ, Ezekowitz MD, Yusuf S, et al. Dabigatran versus warfarin in patients with atrial fibrillation. N Engl J Med. 2009;361(12):1139-1151.

7. Patel MR, Mahaffey KW, Garg J, et al. Rivaroxaban versus warfarin in nonvalvular atrial fibrillation. N Engl J Med. 2011;365(10):883-891.

8. Granger $\mathrm{CB}$, Alexander $\mathrm{JH}, \mathrm{McMurray} \mathrm{JJ}$, et al. Apixaban versus warfarin in patients with atrial fibrillation. N Engl J Med. 2011;365(11):981-992.

9. Chee YL, Crawford JC, Watson HG, Greaves M. Guidelines on the assessment of bleeding risk prior to surgery or invasive procedures. British Committee for Standards in Haematology. Br J Haematol. 2008;140(5):496-504.

10. Suchman AL, Mushlin Al. How well does the activated partial thromboplastin time predict postoperative hemorrhage? Jama. 1986;256(6):750-753.

11. Kitchens CS. To bleed or not to bleed? Is that the question for the PTT? J Thromb Haemost. 2005;3(12):2607-2611.

12. Al Dieri R, Peyvandi F, Santagostino E, et al. The thrombogram in rare inherited coagulation disorders: its relation to clinical bleeding. Thromb Haemost. 2002;88(4):576-582.

13. Gatt A, van Veen JJ, Woolley AM, Kitchen S, Cooper P, Makris M. Thrombin generation assays are superior to traditional tests in assessing anticoagulation reversal in vitro. Thromb Haemost. 2008;100(2):350-355.

14. Altman R, Scazziota A, Herrera L, Gonzalez C. Relationship between thrombin generation and international normalized ratio in patients receiving oral vitamin K antagonist therapy. J Thromb Haemost. 2007;5(7):1552-1569.

15. Gerotziafas GT, Dupont C, Spyropoulos AC, et al. Differential inhibition of thrombin generation by vitamin $\mathrm{K}$ antagonists alone and associated with low-molecular-weight heparin. Thromb Haemost. 2009;102(1):42-48.

16. Wells G, Shea, B, O'Connel, D et al. The Newcastle-Ottawa scale (NOS) for assessing the quailty of nonrandomised studies in meta-analyses. http://www.ohri.ca/programs/clinical_epidemiology/ oxford.htm.

17. Desmurs-Clavel H, Huchon C, Chatard B, Negrier C, Dargaud Y. Reversal of the inhibitory effect of fondaparinux on thrombin generation by rFVIIa, aPCC and PCC. Thromb Res. 2009;123(5):796-798.

18. Zahir H, Brown KS, Vandell AG, et al. Edoxaban effects on bleeding following punch biopsy and reversal by a 4-factor prothrombin complex concentrate. Circulation. 2015;131(1):82-90.

19. Herrmann R, Thom J, Wood A, Phillips M, Muhammad S, Baker R. Thrombin generation using the calibrated automated thrombinoscope to assess reversibility of dabigatran and rivaroxaban. Thromb Haemost. 2014;111(5):989-995.

20. Levi M, Moore KT, Castillejos CF, et al. Comparison of three-factor and four-factor prothrombin complex concentrates regarding reversal of the anticoagulant effects of rivaroxaban in healthy volunteers. J Thromb Haemost. 2014;12(9):1428-1436. 
21. Perzborn E, Heitmeier S, Laux V, Buchmuller A. Reversal of rivaroxaban-induced anticoagulation with prothrombin complex concentrate, activated prothrombin complex concentrate and recombinant activated factor VII in vitro. Thromb Res. 2014;133(4):671-681.

22. Dinkelaar J, Patiwael S, Harenberg J, Leyte A, Brinkman HJ. Global coagulation tests: their applicability for measuring direct factor $\mathrm{Xa}$ - and thrombin inhibition and reversal of anticoagulation by prothrombin complex concentrate. Clin Chem Lab Med. 2014;52(11):1615-1623.

23. Dargaud Y, Desmurs-Clavel H, Marin S, Bordet JC, Poplavsky JL, Negrier C. Comparison of the capacities of two prothrombin complex concentrates to restore thrombin generation in plasma from orally anticoagulated patients: an in vitro study. J Thromb Haemost. 2008;6(6):962-968.

24. Marlu R, Hodaj E, Paris A, Albaladejo P, Cracowski JL, Pernod G. Effect of non-specific reversal agents on anticoagulant activity of dabigatran and rivaroxaban: a randomised crossover ex vivo study in healthy volunteers. Thromb Haemost. 2012;108(2):217-224

25. Skolnick BE, Mathews DR, Khutoryansky NM, Pusateri AE, Carr ME. Exploratory study on the reversal of warfarin with rFVIIa in healthy subjects. Blood. 2010;116(5):693-701.

26. Eerenberg ES, Kamphuisen PW, Sijpkens MK, Meijers JC, Buller HR, Levi M. Reversal of rivaroxaban and dabigatran by prothrombin complex concentrate: a randomized, placebo-controlled, crossover study in healthy subjects. Circulation. 2011;124(14):1573-1579.

27. Khoo TL, Weatherburn C, Kershaw G, Reddel CJ, Curnow J, Dunkley S. The use of FEIBA(R) in the correction of coagulation abnormalities induced by dabigatran. Int J Lab Hematol. 2013;35(2):222-224.

28. Perlstein I, Wang Z, Song $Y$, et al. Reversal of apixaban anticoagulation by 4 -factor prothrombin complex concentrates in healthy subjects. Blood Conference: 56th Annual Meeting of the American Society of Hematology, ASH. 2014;124(21).

29. Evans G, Luddington R, Baglin T. Beriplex P/N reverses severe warfarin-induced overanticoagulation immediately and completely in patients presenting with major bleeding. Br J Haematol. 2001;115(4):998-1001.

30. Cheung KC, Rodgers S, McRae S. Local experience in reversal dabigatran using activated prothrombin complex concentrates (FEIBA). Haematologica. 2012;97:185.

31. Brinkman HJM, Dinkelaar J, Patiwael S, Harenberg J, Leyte A. How should we measure the reversal of direct factor XA or thrombin inhibitors by prothrombin complex concentrate? Journal of Thrombosis and Haemostasis. 2014;12:16.

32. Reijers J, Moerland M, Frieling J, et al. Pharmacodynamic effects of two recombinant FVIla products in anticoagulated healthy volunteers. Journal of Thrombosis and Haemostasis. 2013;11:1129.

33. Godier A, Martin AC, Samama CM, Fischer AM, Le Bonniec B. Reversal of anticoagulant effects of apixaban with non-specific prohaemostatic agents: An in vitro study. Journal of Thrombosis and Haemostasis. 2013;11:826.

34. Brinkman HJA, Herpers R, Van Beem RT, et al. Ex vivo monitoring of Fenprocoumon reversal by prothrombin complex concentrate using thrombography. Journal of Thrombosis and Haemostasis. 2013;11:825.

35. Herpers R, Van Beem RT, Michel WM, et al. INR vs. Thrombin generation assays for guiding VKA reversal: A retrospective comparison. Nederlands Tijdschrift voor Klinische Chemie en Laboratoriumgeneeskunde. 2015;40 (2):107-108.

36. Mullier F, Douxfils J, Chatelain C, Chatelain B, Dogne JM. Thrombin generation assay is superior to traditional coagulometric and chromogenic assays to monitor new oral anticoagulants. Blood Conference: 54th Annual Meeting of the American Society of Hematology, ASH. 2012;120(21).

37. Galan AM, Arellano-Rodrigo E, Veronica Sanz V, et al. Reversal of the antithrombotic action of rivaroxaban and dabigatran: A clinical study in healthy volunteers. Blood Conference: 54th Annual Meeting of the American Society of Hematology, ASH. 2012;120(21). 
38. Pillitteri D, Pilgrimm-Thorp AK, Krause M, Scholz T, Kirchmaier C. Antidotal effects of non-specific reversal agents on anticoagulant-induced inhibition of thrombin generation. Journal of Thrombosis and Haemostasis. 2013;11:562-563.

39. Halim AB, Li Y, Stein E, Mendell J. Low concentrations of rhFVIla or FEIBA significantly and rapidly reverse the anticoagulant effects of supratherapeutic edoxaban. Blood Conference: 53rd Annual Meeting of the American Society of Hematology, ASH. 2011;118(21).

40. Mullier F, Douxfils J, Robert S, et al. Dabigatran: Safety, usefulness and practical details of drug monitoring. Journal of Thrombosis and Haemostasis. 2011;9:857.

41. Mullier F, Douxfils J, Robert S, et al. Rivaroxaban: Safety, usefulness and practical details of drug monitoring. Journal of Thrombosis and Haemostasis. 2011;9:857.

42. Adamidou D, Riddell A, Aghighi S, Bates S, Chowdary P. PRP thrombin generation (TG) in patients on oral anticoagulation with warfarin: Influence of tissue factor (TF) concentration and corn trypsin inhibitor (CTI). Journal of Thrombosis and Haemostasis. 2011;9:590.

43. Voils SA, Martin EJ, Mohammed BM, Bayrlee A, Brophy DF. Laboratory assessment of warfarin reversal with global coagulation tests versus international normalized ratio in patients with intracranial bleeding. Blood Coagul Fibrinolysis. 2015.

44. Schultz NH, Tran HTT, Bjornsen S, Henriksson CE, Sandset PM, Holme PA. The reversal effect of prothrombin complex concentrate (PCC), activated PCC and recombinant activated factor VII against anticoagulation of Xa inhibitor. Thromb J. 2017;15:6.

45. Calmette L, Martin AC, Le Bonniec B, et al. Ticagrelor reversal: in vitro assessment of four haemostatic agents. J Clin Pathol. 2017.

46. Cheung YW, Barco S, Hutten BA, Meijers JC, Middeldorp S, Coppens M. In vivo increase in thrombin generation by four-factor prothrombin complex concentrate in apixaban-treated healthy volunteers. J Thromb Haemost. 2015;13(10):1799-1805

47. Davidson SJ, Burman JF, Philips SM, et al. Correlation between thrombin potential and bleeding after cardiac surgery in adults. Blood Coagul Fibrinolysis. 2003;14(2):175-179.

48. Radulovic $V$, Hyllner $M$, Ternstrom $L$, et al. Sustained heparin effect contributes to reduced plasma thrombin generation capacity early after cardiac surgery. Thromb Res. 2012;130(5):769-774.

49. Schols SE, van der Meijden PE, van Oerle R, Curvers J, Heemskerk JW, van Pampus EC. Increased thrombin generation and fibrinogen level after therapeutic plasma transfusion: relation to bleeding. Thromb Haemost. 2008;99(1):64-70.

50. Schols SE, Lance MD, Feijge MA, et al. Impaired thrombin generation and fibrin clot formation in patients with dilutional coagulopathy during major surgery. Thromb Haemost. 2010;103(2):318-328.

51. Coakley M, Hall JE, Evans C, et al. Assessment of thrombin generation measured before and after cardiopulmonary bypass surgery and its association with postoperative bleeding. J Thromb Haemost. 2011;9(2):282-292.

52. Bosch YP, Al Dieri R, ten Cate $\mathrm{H}$, et al. Measurement of thrombin generation intra-operatively and its association with bleeding tendency after cardiac surgery. Thromb Res. 2014;133(3):488-494.

53. Percy $\mathrm{CL}$, Jones $\mathrm{M}$, Mehta $\mathrm{D}$, et al. Thrombin generation and post-operative bleeding in patients undergoing surgery requiring cardiopulmonary bypass. Journal of Thrombosis and Haemostasis. 2013;11:578.

54. Feder SS, A; Correia, C; Lehmann, S; Meyer, A; Garbade, J; Misfeld, M; Bakhtiary, F; Mohr, F-W; Oberbach, A. Clinical application of thrombin generation: A deeper reflection of plasmatic haemostasis of ventricular assist device recipients. Thoracic and Cardiovascular Surgeon. 2015;63:no pagination.

55. Bloemen S, Zwaveling S, Ten Cate H, Ten Cate-Hoek A, de Laat B. Prediction of bleeding risk in patients taking vitamin K antagonists using thrombin generation testing. PLoS One. 2017;12(5):e0176967. 
56. Luna-Zaizar H, Gonzalez-Moncada Al, Padilla-Lopez EL, et al. Thrombin generation and international normalized ratio in inherited thrombophilia patients receiving thromboprophylactic therapy. Thromb Res. 2015;136(6):1291-1298.

57. Marchetti M, Giaccherini C, Lerede T, Maggioni A, Cefis M, Falanga A. Characterization of thrombin generation (TG) by the calibrated automated thrombography (CAT) in atrial fibrillation (AF) patients on permanent oral anticoagulation therapy (OAT) with warfarin. Journal of Thrombosis and Haemostasis. 2013;3):59-60.

58. Choi Q, Kim JE, Hyun J, Han KS, Kim HK. Contributions of procoagulants and anticoagulants to the international normalized ratio and thrombin generation assay in patients treated with warfarin: potential role of protein $\mathrm{Z}$ as a powerful determinant of coagulation assays. Thromb Res. 2013;132(1):e70-75.

59. Hemker HC, Giesen $\mathrm{P}, \mathrm{Al}$ Dieri R, et al. Calibrated automated thrombin generation measurement in clotting plasma. Pathophysiol Haemost Thromb. 2003;33(1):4-15

60. Verzeroli C, Giaccherini C, Marchetti M, Milesi V, Barcella L, Falanga A. Thrombin generation (TG) potential in patients on chronic oral anticoagulant therapy (OAT). Thrombosis Research. 2014;2):S98-S99.

61. Dargaud $Y$, Hoffman $M$, Lefrapper $L$, et al. Bleeding risk in warfarinized patients with a therapeutic international normalized ratio: the effect of low factor IX levels. J Thromb Haemost. 2013;11(6):1043-1052.

62. Ninivaggi M, Apitz-Castro R, Dargaud Y, de Laat B, Hemker HC, Lindhout T. Whole-blood thrombin generation monitored with a calibrated automated thrombogram-based assay. Clin Chem. 2012;58(8):1252-1259.

63. Hemker HC, Al Dieri R, De Smedt E, Beguin S. Thrombin generation, a function test of the haemostatic-thrombotic system. Thromb Haemost. 2006;96(5):553-561.

64. van Veen JJ, Gatt A, Makris M. Thrombin generation testing in routine clinical practice: are we there yet? $\mathrm{Br} \mathrm{J}$ Haematol. 2008;142(6):889-903.

65. Curvers J, Thomassen MC, Rimmer J, et al. Effects of hereditary and acquired risk factors of venous thrombosis on a thrombin generation-based APC resistance test. Thromb Haemost. 2002;88(1):5-11.

66. Besser M, Baglin C, Luddington R, van Hylckama Vlieg A, Baglin T. High rate of unprovoked recurrent venous thrombosis is associated with high thrombin-generating potential in a prospective cohort study. J Thromb Haemost. 2008;6(10):1720-1725.

67. Tripodi A, Legnani C, Chantarangkul V, Cosmi B, Palareti G, Mannucci PM. High thrombin generation measured in the presence of thrombomodulin is associated with an increased risk of recurrent venous thromboembolism. J Thromb Haemost. 2008;6(8):1327-1333.

68. Gatt A, van Veen JJ, Bowyer A, et al. Wide variation in thrombin generation in patients with atrial fibrillation and therapeutic International Normalized Ratio is not due to inflammation. Br J Haematol. 2008;142(6):946-952.

69. Veeger NJ, Piersma-Wichers M, Meijer K, Hillege HL. Minor bleeds alert for subsequent major bleeding in patients using vitamin K antagonists. Br J Haematol. 2011;153(4):508-514.

70. van Rein N, le Cessie S, van Vliet IP, et al. Increased risk of major bleeding after a minor bleed during treatment with vitamin K antagonists is determined by fixed common risk factors. J Thromb Haemost. 2016;14(5):948-952

71. van der Meijden PE, Bouman AC, Feijge MA, et al. Platelet dysfunction in thrombosis patients treated with vitamin K antagonists and recurrent bleeding. PLoS One. 2013;8(5):e64112.

72. Wagenvoord RJ, Deinum J, Elg M, Hemker HC. The paradoxical stimulation by a reversible thrombin inhibitor of thrombin generation in plasma measured with thrombinography is caused by alpha-macroglobulin-thrombin. J Thromb Haemost. 2010;8(6):1281-1289.

73. Gribkova IV, Lipets EN, Rekhtina IG, et al. The modification of the thrombin generation test for the clinical assessment of dabigatran etexilate efficiency. Sci Rep. 2016;6:29242.

74. Dale B, Eikelboom JW, Weitz JI, et al. Dabigatran attenuates thrombin generation to a lesser extent than warfarin: could this explain their differential effects on intracranial hemorrhage and myocardial infarction? J Thromb Thrombolysis. 2013;35(2):295-301. 


\section{SUPPLEMENTAL INFORMATION}

\section{S1. Newcastle - Ottawa Quality Assessment Scale case control studies}

Note: A study can be awarded a maximum of one star for each numbered item within the Selection and Exposure categories. A maximum of two stars can be given for Comparability.

\section{Selection}

1. Is the case definition adequate?
a) yes, with independent validation $\star$
b) yes, eg record linkage or based on self reports
c) no description

2. Representativeness of the cases
a) consecutive or obviously representative series of cases $\star$
b) potential for selection biases or not stated

3. Selection of Controls
a) community controls $\star$
b) hospital controls
c) no description

4. Definition of Controls
a) no history of disease (endpoint) *
b) no description of source 


\section{Comparability}

1. Comparability of cases and controls on the basis of the design or analysis
a) study controls for (Select the most important factor.) «
b) study controls for any additional factor $\star$ (This criteria could be modified to indicate specific control for a second important factor.)

\section{Exposure}

1. Ascertainment of exposure
a) secure record (eg surgical records) ᄎ
b) structured interview where blind to case/control status $\star$
c) interview not blinded to case/control status
d) written self report or medical record only
e) no description

2. Same method of ascertainment for cases and controls
a) yes $\star$
b) no

3. Non-Response rate
a) same rate for both groups $\star$
b) non respondents described
c) rate different and no designation 


\section{S2. Newcastle - Ottawa Quality Assessment Scale cohort studies}

Note: A study can be awarded a maximum of one star for each numbered item within the Selection and Outcome categories. A maximum of two stars can be given for Comparability

\section{Selection}

1. Representativeness of the exposed cohort
a) truly representative of the average (describe) in the community *
b) somewhat representative of the average in the community
c) selected group of users e.g. nurses, volunteers
d) no description of the derivation of the cohort

2. Selection of the non exposed cohort
a) drawn from the same community as the exposed cohort $\star$
b) drawn from a different source
c) no description of the derivation of the non exposed cohort

3. Ascertainment of exposure
a) secure record (eg surgical records) ᄎ
b) structured interview $\star$
c) written self report
d) no description

4. Demonstration that outcome of interest was not present at start of study
a) yes $\star$
b) no 


\section{Comparability}

1. Comparability of cohorts on the basis of the design or analysis
a) study controls for (select the most important factor) *
b) study controls for any additional factor $\star$ (This criteria could be modified to indicate specific control for a second important factor.)

\section{Outcome}

1. Assessment of outcome
a) independent blind assessment $\star$
b) record linkage $\star$
c) self report
d) no description

2. Was follow-up long enough for outcomes to occur
a) yes (select an adequate follow up period for outcome of interest)
b) no

3. Adequacy of follow up of cohorts
a) complete follow up - all subjects accounted for $\star$
b) subjects lost to follow up unlikely to introduce bias - small number lost - > (select an adequate \%) follow up, or description provided of those lost) *
c) follow up rate <____. (select an adequate \%) and no description of those lost
d) no statement 


\section{S3. Table 3.}

To be able to classify the quality of the studies, we defined the amount of stars as shown in the table.

\begin{tabular}{lll} 
Stars $(\star)$ & $(\%)$ & Quality \\
\hline $1-3$ & $<35 \%$ & poor \\
$4-6$ & $35-70 \%$ & moderate \\
$7-9$ & $70-100 \%$ & good
\end{tabular}

\section{S4. Methodological aspects of the included studies}

The Newcastle-Ottawa Quality Assessment Scale was used to assess the methodological quality of both the cohort studies and the case-control study. ${ }^{1}$ The studies by respectively Bloemen, Luna-Záizar, Marchetti, Choi and Vezeroli et al. are all prospective cohort studies.

\section{Cohort studies:}

Bloemen, Luna-Záizar, Choi et al. investigated a representative selection of patients treated with VKAs in the community. Whether these patients were consecutively included is not mentioned. Marchetti and Vezeroli selected patients who were in their appropriate INR range, and had similar indications for OAC treatment. The selection of the non-exposed cohort in all investigated cohort studies was drawn from the same community as the exposed cohort. Ascertainment of exposure to VKAs was also obtained by all cohort studies, as inclusion was mostly dependent on INR-values. None of the authors demonstrated that the outcome of interest (bleeding) was not present at the start of the study.

Bloemen et al. investigated the influence of sex, age and co-medication (including platelet inhibitors) and additionally controlled for individual coagulation factors, natural anticoagulation factors and other biological proteins (e.g. haemoglobin, haematocrit and fibrinogen). Marchetti et al. controlled for at least two confounding factors (sex and age), while Vezeroli et al. controlled for age only. Other important confounding factors like co-medication are not described by these studies. Furthermore Luna-Záizar and Choi et al. do not mention an implemented correction for confounding factors at all. Choi et al. excluded patients that used other anticoagulant therapy as co-medication. In this way the authors eliminated one important factor that could have influenced the results. 
The assessment of outcome is not described in two of the five studies (Marchetti and Vezeroli). Bloemen et al. identified outcome by record linkage, using codes for specific bleeding complications. Bleedings could be reported by general practitioners, hospitals or by patients themselves. The length of follow-up was acceptable (fifteen months). Luna-Záizar and Choi et al. collected outcome via record linkage as well, unfortunately the length of follow-up was not described. Vezeroli and Marchetti et al. do not explain how they established outcome, but did perform follow-up for an adequate period (1 and 2 years respectively). The adequacy of follow up of the cohorts was not described for any of the studies.

\section{Case control studies:}

Herpers et al. clearly stated inclusion criteria. Cases were adequately defined and derived from a community of patients using VKAs during routine venepunctures at the hospital. All patients had to sign informed consent to be included, there we no other exclusion criteria. Whether patients were consecutively selected, was not described. It was not described if patients had a history of bleeding. Control for confounding factors is not mentioned by the authors. Ascertainment of exposure was obtained through secure medical records of structured interview in the hospital at moment of inclusion. The same method to assess exposure was used in cases and controls. Patients would come to the hospital for the reversal of their VKAs. This could be either bleeding or upcoming surgery. Bleeding was fairly defined, (cerebral $50 \%$, gastro-intestinal $20 \%$ or other $30 \%$ ). The non-response rate was in this case not applicable, because cases were selected based on the presence of the outcome. How many patients declined to give informed consent is unknown.

Dargaud et al. performed a cross-sectional study in which physicians adequately defined the cases of haemorrhage when consecutive patients arrived at the emergency department. Truly representative cases were investigated, drawn from the community of patients treated with VKAs. Whether patients or controls had a history of bleeding was not reported. The outcome of interest is present at the start of the study. Actual control for confounding factors is not mentioned in the paper, but patients using co-medication predisposing for bleeding, liver disease or disseminated intravascular coagulation (DIC) were excluded. In that way the authors eliminated important influential factors. 
Ascertainment of exposure was obtained through secure medical records of structured interview on the emergency department. Cases would present themselves on the emergency department with bleeding complications, while controls would also attend the emergency department, but for other reasons than bleeding. As in the study of Herpers et al., the non-response rate was in this case not applicable, because cases were selected base on the presence of the outcome. Also the number of patients that declined to give informed consent is unknown. The methodological quality of the included studies on the Newcastle-Ottawa Quality assessment scale is represented in table 3a and 3b in the original article.

\section{REFERENCES}

1. Wells G, Brodsky L, O'Connell D, et al. An evaluation of the Newcastle Ottawa Scale: an assessment tool for evaluating the quality of non-randomized studies. Paper presented at: XI International Cochrane Colloquium Book of Abstracts2003. 


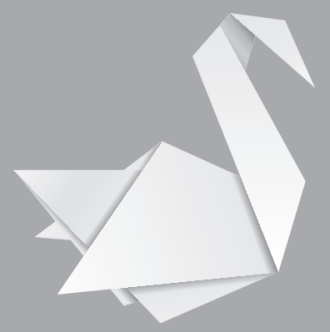




\section{CHAPTER}

The anticoagulant effect of dabigatran is reflected in the lag time and time-to-peak, but not in the endogenous thrombin potential or peak, of thrombin generation

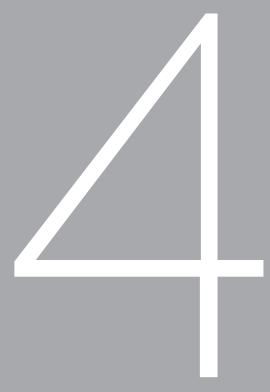

Saartje Bloemen Suzanne Zwaveling Jonathan Douxfils Mark Roest Romy Kremers

François Mullier

Thromb Res. 2018 Oct 6;171:160-166. 


\section{ABSTRACT}

\section{Introduction}

Calibrated automated thrombinography (CAT) is a sensitive method to assess coagulation. Dabigatran inhibits both free thrombin and the a2macroglobulin (a2M)-thrombin complex, which results in an erroneously increased peak and endogenous thrombin potential (ETP) without affecting lag time and time-to-peak. The aim of this study was to elucidate the artefacts in CAT when dabigatran is present.

\section{Materials and Methods}

Thrombin generation (TG) was measured in vitro by using CAT in the presence or absence of $6 \mu \mathrm{M}$ idarucizumab in plasma spiked with dabigatran. Additionally, ex vivo measurements were performed in plasmas of 63 patients using dabigatran in the presence and absence of idarucizumab.

\section{Results}

The in vitro experiments confirmed that the ETP, peak and velocity index were artificially increased. This was mainly due to the inhibition of the calibrator by dabigatran and partly due to CAT algorithms. The calibration artefact could be resolved by adding idarucizumab to the calibrator well. However, the second, mathematical artefact remains when dabigatran is present in the TG well. These findings were corroborated by ex vivo experiments i.e. the lag time and time-to-peak were significantly reduced in patients upon addition of idarucizumab, but the ETP and peak were not significantly affected. The velocity index did change significantly, since this is a combination of time-dependent factors and the peak.

\section{Conclusions}

The peak, ETP and velocity index do not represent the anticoagulant effect of dabigatran on TG measured with CAT. The lag time and time-to-peak, however, do reflect the effect of dabigatran 


\section{INTRODUCTION}

Dabigatran is a direct oral anticoagulant (DOAC) inhibiting thrombin. Dabigatran and DOACs inhibiting factor Xa (rivaroxaban, apixaban, edoxaban) have been shown to be at least non-inferior to vitamin $\mathrm{K}$ antagonists (VKAs) with respect to the prevention and treatment of venous thrombosis. Additionally, large clinical trials have shown less severe haemorrhage during DOAC treatment compared to treatment with VKAs. ${ }^{1-7}$ However, bleeding remains the main concern during DOAC treatment and severe bleeding does still occur, which makes reversal therapy necessary. For the reversal of dabigatran, the humanized monoclonal antibody fragment idarucizumab (Praxbind $\AA$, Boehringer Ingelheim) was developed. ${ }^{8}$ Idarucizumab binds both free and thrombin-bound dabigatran in a 1:1 ratio with an affinity 350 times stronger than the affinity of dabigatran for thrombin. ${ }^{8}$ Studies have shown that administration of idarucizumab successfully restores coagulation. ${ }^{8,9}$ Measuring the effect of dabigatran around administration of idarucizumab is needed, not only to evaluate reversal, but also to assess the remaining bleeding risk of patients. ${ }^{10-12}$ Measuring dabigatran levels before administration helps to select patients who would benefit from reversal thus avoiding unnecessary administration of expensive reversal agents. Measuring after reversal could help detect patients with a rebound effect of plasma dabigatran levels. Moreover, monitoring the effect of dabigatran on coagulation could detect an underlying coagulopathy unrelated to treatment. ${ }^{13-15}$ This can only be achieved by reliable assays that provide information on the global coagulation status.

At the moment, few sensitive methods are available to measure the dabigatran concentration and consequently the neutralizing effect of idarucizumab. Mass spectrometry, the diluted thrombin time (e.g. the Hemoclot thrombin inhibitor ${ }^{\circledR}(\mathrm{HTI})$, Hyphen BioMed), the ecarin clotting assay as well as the ecarin chromogenic assay (e.g. STA-ECA II, Stago) can be used to determine drug levels of dabigatran. ${ }^{16-19}$ However, none of these assays are able to provide information on the differential effect of dabigatran on coagulation or on endogenous coagulopathies. Thrombin generation (TG) is an assay that provides insight in the global coagulation characteristics of patients, and it was shown that TG parameters are associated with both bleeding and thrombotic tendencies in various patient populations. ${ }^{20-23}$ Some studies have been carried out towards the effect dabigatran or other reversible, direct thrombin inhibitors on TG. Dabigatran, as expected, prolongs the lag time and time-to-peak, but counterintuitively, increases the peak height and endogenous thrombin potential (ETP). ${ }^{24-26}$ 
We postulate that these artefacts are due to the calculations used in calibrated automated thrombinography (CAT) ${ }^{27}$ and have to be elucidated and solved for CAT to become a clinical tool able to assess patients using direct thrombin inhibitors. Thrombin bound to a2macroglobulin (a2M) can still cleave small molecules such as the fluorescent substrate used in CAT. ${ }^{20}$ Additionally, direct thrombin inhibitors, such as dabigatran, can also inhibit thrombin in complex with a2M. In order to discern the effects of dabigatran on CAT measurements and calculations, one needs to understand which algorithms are applied in the conversion of the fluorescent signal to the final concentration of free thrombin. By determining the first derivative of the fluorescent data and comparing the slope of the TG curve to that of the calibrator curve, the total thrombin concentration can be determined. This is the first part of the calculations that is affected by dabigatran. The second algorithm is the calculation of a2M-thrombin present in the measurement (TG) well. After the conversion of the fluorescence the total thrombin concentration is represented. In order to obtain the concentration of free thrombin, the a2M-thrombin needs to be subtracted from the total amount of thrombin. ${ }^{28}$ We assume that in the presence of dabigatran this is the second part of the calculations that could be disturbed. Therefore, unlike what previous reports ${ }^{25,26}$ have stated, we postulate that dabigatran not only affects the calibrator, but also the correction for the signal caused by a2M-thrombin in the measurement well. The aim of the current study was to situate and clarify the artefacts (increased ETP and peak) found in CAT when measuring samples containing dabigatran, as well as to investigate to what level these artefacts could be corrected by idarucizumab.

\section{MATERIALS \& METHODS}

\section{Plasma samples}

Sixty-three plasma samples from patients treated with dabigatran etexilate for stroke prevention in non-valvular atrial fibrillation were included in the study. The sample collection was performed in accordance with the Declaration of Helsinki and was approved by the Medical Ethics Committee of the Centre Hospitalier Universitaire UCL Namur in Yvoir, Belgium (number of approval BU3920096633). Blood was taken by venipuncture from the antecubital vein and collected into $0.109 \mathrm{M}$ sodium citrate $(9: 1 \mathrm{~V} / \mathrm{V})$ tubes (Venosafe ${ }^{\circledR}$, Terumo, Heverlee, Belgium) using a 21-gauge needle (Terumo). Platelet poor plasma (PPP) was obtained from the supernatant fraction after double centrifugation at $1,500 \mathrm{~g}$ for 15 
min at room temperature. Afterwards, plasma was aliquoted and frozen at $-80^{\circ} \mathrm{C}$ without delay. Frozen plasma sample aliquots were thawed and heated to $37^{\circ} \mathrm{C}$ for $5-10$ min before experiments. Normal pooled plasma (NPP) was prepared from blood collected from 116 healthy volunteers. Plasma was pooled after an initial centrifugation step (2,500 g, 5 min), followed by ultracentrifugation at 100,000 g for $10 \mathrm{~min}$. Aliquots of $500 \mu \mathrm{l}$ were snap frozen in liquid nitrogen and stored at $-80^{\circ} \mathrm{C}$ until analysis.

\section{Reagents}

Synthetic phospholipids (PL) were obtained from Avanti Polar Lipids Inc. (Alabaster, AL, USA) and used as vesicles consisting of phosphatidylserine, phosphatidylethanolamine and phosphatidylcholine (1:1:3, mol:mol:mol). Recombinant tissue factor (TF) was Innovin ${ }^{8}$ (Dade-Behring, Marburg, Germany). The fluorogenic substrate Z-Gly-Gly-Arg-aminomethylcoumarin (ZGGR-AMC) was purchased from Bachem (Basel, Switzerland). The calibrator (a2M-thrombin) was prepared as described by Hemker et al. ${ }^{29}$ Hepes buffers containing 5 $\mathrm{mg} / \mathrm{ml}$ or $60 \mathrm{mg} / \mathrm{ml}$ bovine serum albumin (BSA5 and BSA60) were prepared according to Hemker et al. ${ }^{30}$ Dabigatran was purchased from Alsachim (Illkirch Graffenstaden, France) and idarucizumab (Praxbind $®$ ) was from Boehringer Ingelheim (Ingelheim am Rhein, Germany).

\section{Calibrated automated thrombinography}

TG was performed in duplicate in platelet poor plasma using CAT as described previously by Hemker et al. ${ }^{29}$ The calibrator wells contained a final concentration of a2M-thrombin equivalent to $100 \mathrm{nM}$ thrombin activity. The final concentration of TF for the in vitro experiments was 1, 5 or $20 \mathrm{pM}$ added together with $4 \mu \mathrm{M}$ PL in the TG well. The patient samples were measured at $5 \mathrm{pM}$ TF and $4 \mu \mathrm{M} \mathrm{PL}$. These samples were measured in the presence of $6 \mu \mathrm{M}$ of idarucizumab in the calibrator wells and in presence and absence of $6 \mu \mathrm{M}$ idarucizumab (final concentration) in the TG well. This concentration was chosen since it can neutralize concentrations of dabigatran up to $6 \mu \mathrm{M}$, i.e. $2,830 \mathrm{ng} / \mathrm{ml}$ (since the idarucizumab binds dabigatran in a 1:1 ratio) and is expected to cover the range of concentrations expected in this population. Additionally, the mean idarucizumab concentration in the REVERSE-AD study after $4 \mathrm{~h}$ was around 5-6 $\mu \mathrm{M} .{ }^{9}$ Data were analysed with specialized software from Thrombinoscope (Maastricht, The Netherlands). The velocity index was calculated as: peak/ (time-to-peak - lag time). a2M-thrombin concentration curves were deducted from the raw, total thrombin fluoresence data as described in Hemker et al. ${ }^{28}$ 


\section{Dabigatran concentration determination}

The dabigatran concentration was determined from the inhibition of a2M-thrombin activity by the dabigatran present in a plasma sample. Samples were prediluted in Hepes buffers containing $5 \mathrm{mg} / \mathrm{ml}$ BSA. The diluted samples were incubated (in duplicate) with $30 \mathrm{nM}$ and $70 \mathrm{nM}$ a2M-thrombin and the remaining a2M-thrombin activity was measured kinetically as the cleavage of thrombin substrate ZGGR-AMC on a Fluoroskan Ascent fluorimeter (Thermo Labsystems, Helsinki, Finland). The inhibition of a2M-thrombin was compared to a dose-response series of dabigatran to enable the determination of the plasma dabigatran level. Dabigatran levels between 50-500 nM (i.e. 23.6-236 ng/ml) were calculated based on the reference curve containing $30 \mathrm{nM}$ a2M-thrombin and levels above $500 \mathrm{nM}$ (i.e. $236 \mathrm{ng} / \mathrm{ml}$ ) were calculated from the curve containing $70 \mathrm{nM}$ a2M-thrombin. Dilute thrombin time using Hemoclot Thrombin Inhibitors ${ }^{\circledR}$ (Hyphen BioMed®) was also performed for all samples on the STA-R Evolution ${ }^{\circledR}$ coagulometer (Diagnostica Stago $\left.{ }^{\circledR}\right)$, as previously described. ${ }^{19}$

\section{Statistical analysis}

Statistical analyses were performed with Graphpad Prism software (version 5.00). Normality of the data was assessed using the Shapiro-Wilk test. Data are represented as median with interquartile range (IQR). The Wilcoxon signed rank test was used to determine the difference between patient samples before and after addition of idarucizumab. A two-sided p-value of 0.05 was considered statistically significant. Data are represented as mean with standard deviation (SD).

\section{RESULTS}

\section{Inaccurate ETP, peak and velocity index in the presence of dabigatran}

TG was measured by the CAT method in normal pooled plasma spiked with 0-1,000 nM dabigatran, which corresponds to $0-472 \mathrm{ng} / \mathrm{ml}$ (Figure 1). Dabigatran (present in both calibrator and TG wells) caused a counter-intuitive increase of the peak height and the ETP (Figure 1C-F), since a reduction of these parameters would be expected. The minimal and maximal values of the peak at 1 pM TF were $123 \mathrm{nM}$ (in the absence of dabigatran) versus 2,142 nM (500 nM dabigatran) and $263 \mathrm{nM}$ (in the absence of dabigatran) versus 3,625 nM (700 nM dabigatran) at 5 pM TF; for the ETP these were 1,064 nM.min (in the absence of dabigatran) versus 13,478 nM.min (500 nM dabigatran) at 1 pM TF and 1,159 nM.min (in the absence of dabigatran) 

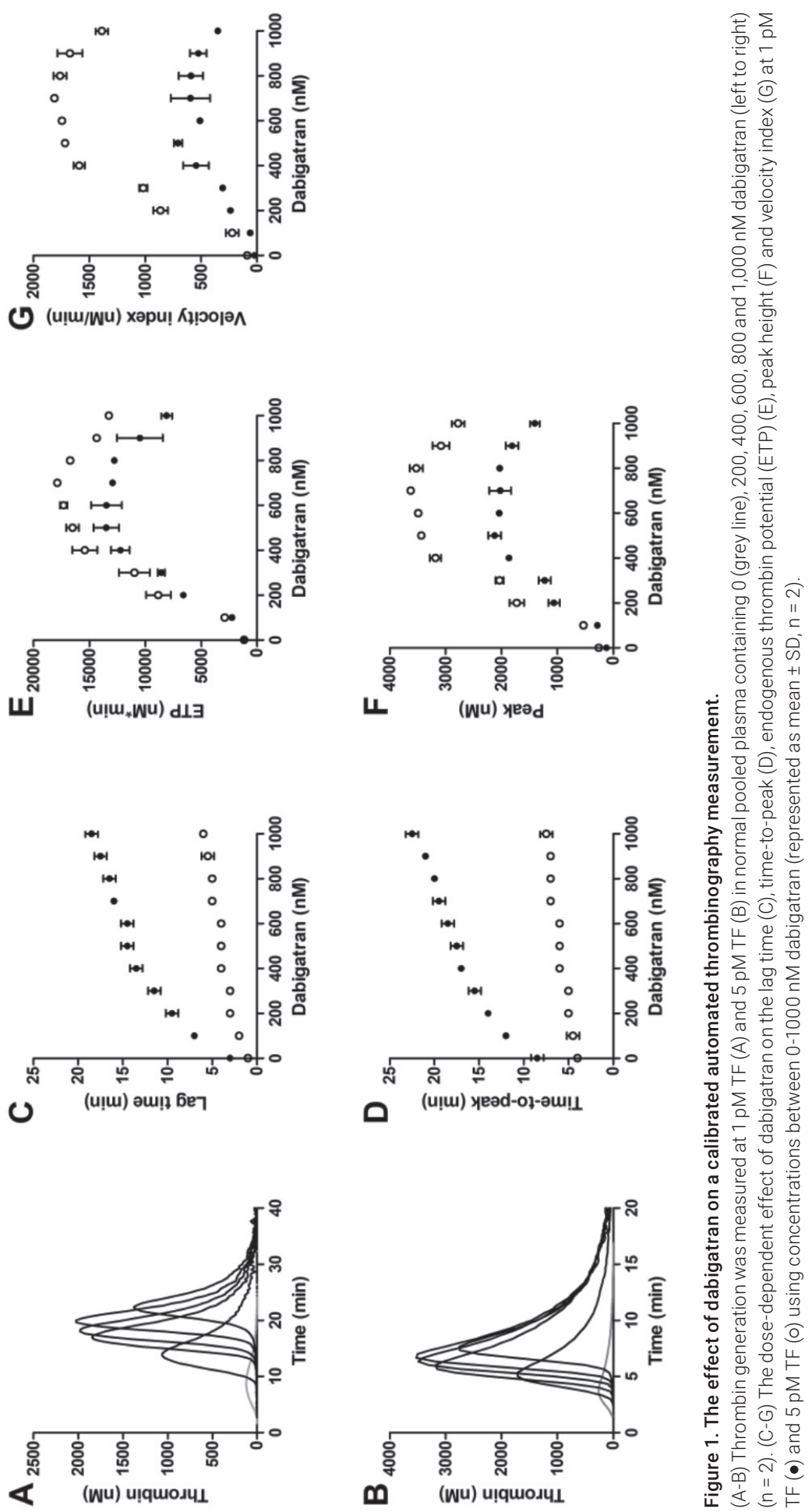

لI $\left(u ! u_{*} w u\right) d \perp \exists$

$$
\text { ᄂ } \text { L }^{\text {(wu) reed }}
$$

$\mathcal{O}$ (u!̣) omị be 
versus 17,302 nM.min (700 nM dabigatran) at $5 \mathrm{pM}$ TF; and for the velocity index these were $22 \mathrm{nM} / \mathrm{min}$ (in the absence of dabigatran) versus $708 \mathrm{nM} / \mathrm{min}$ (500 nM dabigatran) at $1 \mathrm{pM}$ TF and $88 \mathrm{nM} / \mathrm{min}$ (in the absence of dabigatran) versus 1,812 nM/min (700 nM dabigatran) at 5 pM TF. The anticoagulant effect of dabigatran is reflected in the prolonged lag time (6.2 times and 6 times) and time-to-peak (2.6 times and 1.9 times) at both 1 and 5 pM TF, respectively.

Dabigatran reduces the activity of the TG calibrator on the fluorescent substrate dose-dependently (Figure 2). The addition of $6 \mu \mathrm{M}$ idarucizumab to the calibrator well is sufficient to neutralize the inhibitory effect of at least $1,000 \mathrm{nM}(472 \mathrm{ng} / \mathrm{ml})$ of- dabigatran.
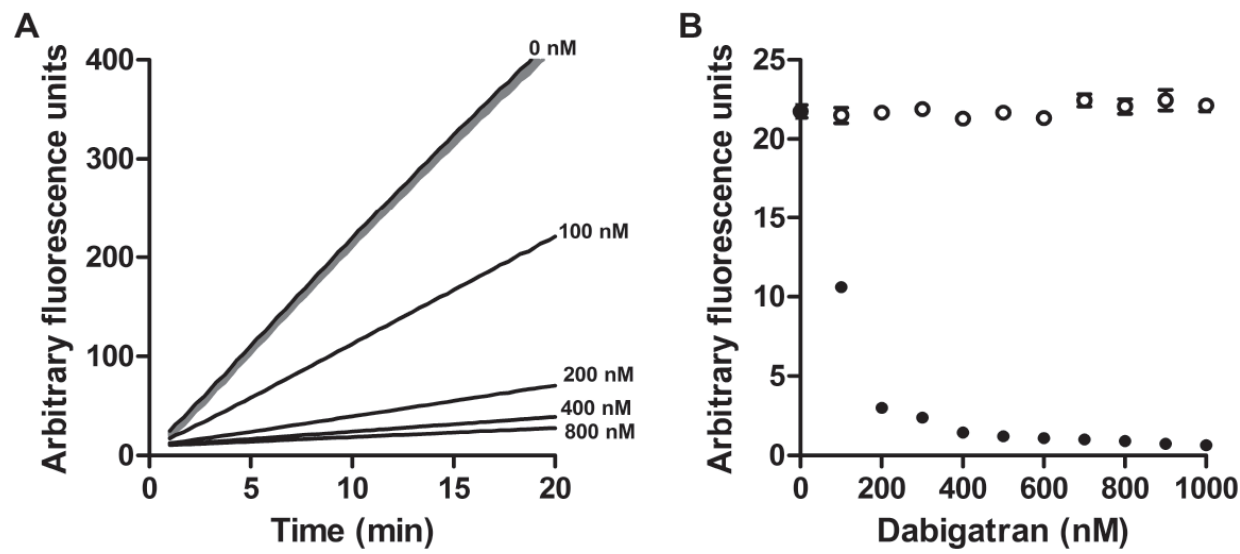

Figure 2. The effect of dabigatran and idarucizumab on the fluorescent activity of the calibrator.

(A) The effect of different dabigatran concentrations on the measurement of the calibrator curves in the absence (black) and presence of $6 \mu \mathrm{M}$ idarucizumab (grey). The curves containing different dabigatran concentrations in the presence of idarucizumab are all overlapping $(n=2)$. (B) Quantification of the dose-dependent inhibition of the thrombin generation calibrator by dabigatran (0-1,000 nM) in the absence $(\bullet)$ and presence of idarucizumab (o) (represented as mean $\pm S D, n=2$ ).

ETP, peak and velocity index remain inaccurate after neutralization of dabigatran in calibrator wells

The addition of $6 \mu \mathrm{M}$ idarucizumab to the calibrator well enables the correct measurement of the calibrator activity in samples containing dabigatran (Figure 3). However, at lower dabigatran concentrations (<300 nM (142 ng/ml)), a stimulatory effect of dabigatran on the TG peak height remains, particularly at the lower tissue factor concentration tested (Figure 3A). At 1 pM tissue factor the peak without addition was $121 \mathrm{nM}$, this increased to maximally $156 \mathrm{nM}$ at $200 \mathrm{nM}$ dabigatran (28\%). In the presence of $1,000 \mathrm{nM}$ of dabigatran the peak was $44 \mathrm{nM}$. At $5 \mathrm{pM}$ TF the increase was only detectable at $100 \mathrm{nM}$ dabigatran with a peak value of $275 \mathrm{nM}$ as compared to 
$264 \mathrm{nM}$ in the absence of dabigatran. At 1,000 nM dabigatran the peak was $90 \mathrm{nM}$. The ETP and velocity index follow the same pattern, at 1 pM TF there was an increase from 1099 nM.min (no dabigatran) to $1320 \mathrm{nM} \cdot \min$ (200 nM dabigatran) for the ETP and from $19 \mathrm{nM} / \mathrm{min}$ (no dabigatran) to $38 \mathrm{nM} / \mathrm{min}(100 \mathrm{nM})$ dabigatran for the velocity index. At $5 \mathrm{pM}$ TF the ETP increased from 1276 nM.min (no dabigatran) to 2050 nM.min (100 nM dabigatran), the velocity index, however, gradually decreased from $132 \mathrm{nM} / \mathrm{min}$ (no dabigatran) to $79 \mathrm{nM} / \mathrm{min}$ (1,000 nM dabigatran).

A

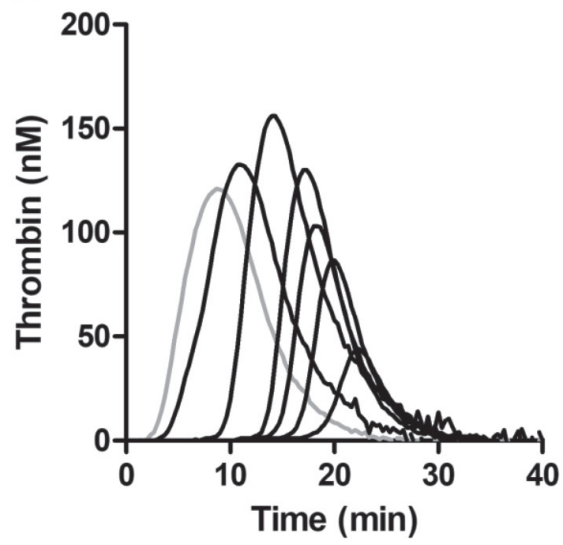

B

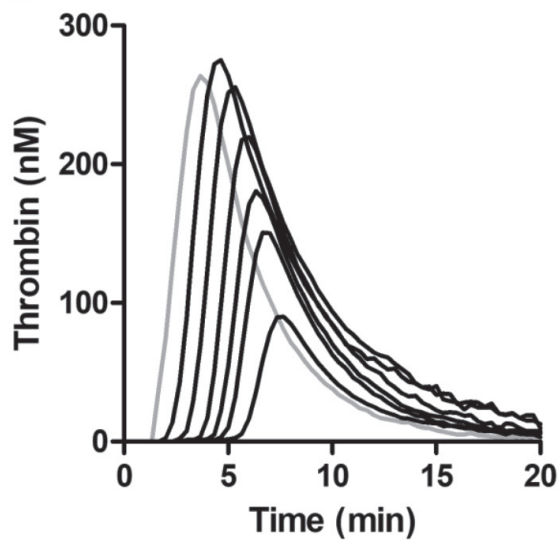

Figure 3. Thrombin generation curves after a correction of the calibration, achieved by addition of idarucizumab $(6 \mu \mathrm{M})$ to the calibrator wells.

Thrombin generation in normal pooled plasma spiked with 0 (grey line), 100, 200, 400, 600, 800, and 1,000 nM dabigatran (left to right) measured at 1 pM TF (A) and 5 pM TF $(B)(n=2)$.

\section{Neutralizing dabigatran in both calibrator and TG wells allows measurement of 'uninhibited' TG}

Idarucizumab can also be used to neutralize dabigatran in the TG well itself. Figure 4 shows the neutralization of 100 and $400 \mathrm{nM}$ dabigatran in normal pooled plasma by $6 \mu \mathrm{M}$ idarucizumab during TG. Both doses of dabigatran prolong the lag time and time-to-peak, which is restored upon the addition of idarucizumab. The addition of idarucizumab alone to normal pooled plasma does not affect TG (figure 5). When TG is initiated with 1 pM TF, the increase of the peak at the low dabigatran (here $100 \mathrm{nM}$ ) concentration is the most visible. With increasing TF concentrations this effect diminishes and is no longer visible at 20 pM TF. However, upon examination of the a2M-thrombin levels during the TG reaction, it is clear that this complex is inhibited by dabigatran from low to high TF levels (figure 4D-F). Therefore, a lower a2M-thrombin concentration is subtracted from the total thrombin concentration and consequently the ETP and peak are overestimated. Since the velocity index is a function of the peak, this will also be overestimated. 

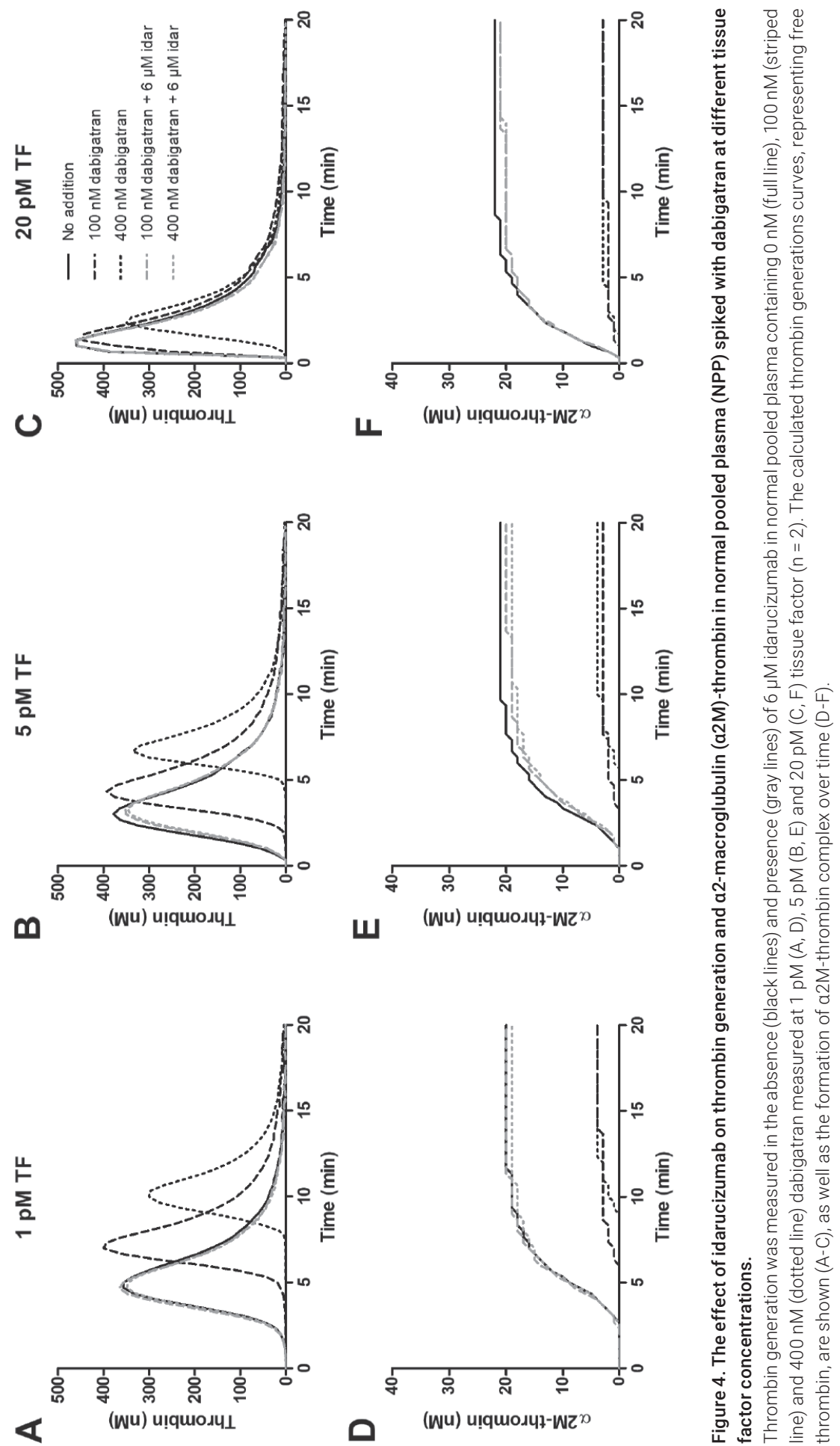

Ш (wu) u!quodul-wZx

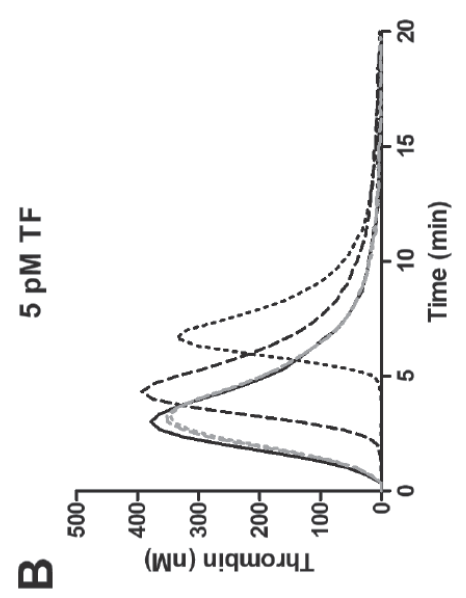

L

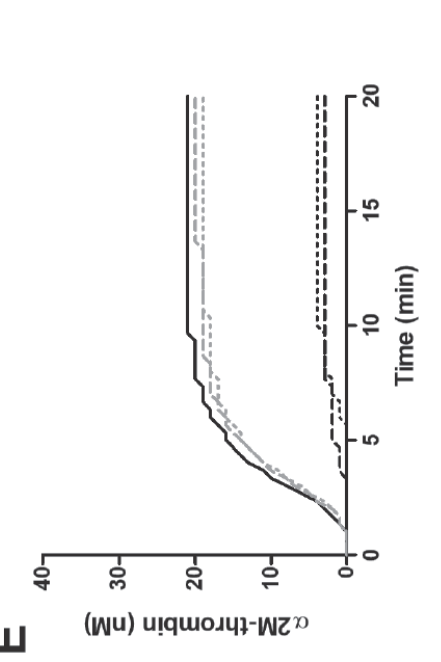




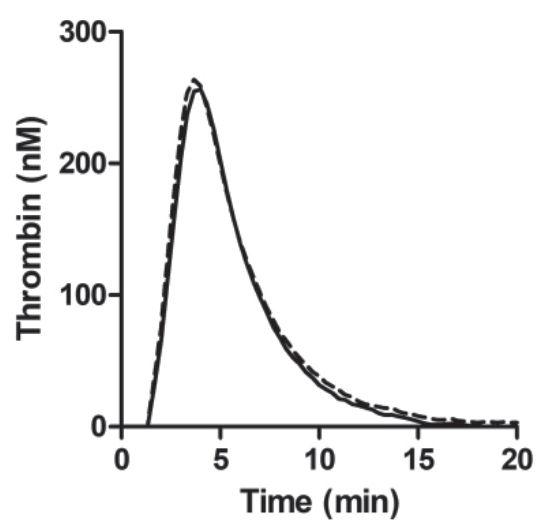

Figure 5. Thrombin generation in the presence and absence of idarucizumab in the absence of dabigatran. Thrombin generation was measured in the presence (full line) and absence (interrupted line) of idarucizumab at 5 pM tissue factor.

\section{Proof of principle in patient samples}

The use of idarucizumab to reverse the effect of dabigatran was tested in a group of patients treated with dabigatran etexilate $(n=63)$. The time since last intake was available for 50 of the patients and the median [IQR] time was $17 \mathrm{~h}[3 \mathrm{~h}-72 \mathrm{~h}]$ with a minimum of $0 \mathrm{~h}$ and a maximum of $540 \mathrm{~h}$. The amount of dabigatran in the patient samples ranged from $0 \mathrm{nM}(0 \mathrm{ng} / \mathrm{ml})$ to $1,362 \mathrm{nM}(643 \mathrm{ng} / \mathrm{ml})$, with a median [IQR] concentration of $205 \mathrm{nM}$ [113 nM-440 nM] (97 $\mathrm{ng} / \mathrm{ml}[53 \mathrm{ng} / \mathrm{ml}-208 \mathrm{ng} / \mathrm{ml}]$ ). As was demonstrated in NPP, the addition of idarucizumab shortens the lag time and time-to-peak in patients with higher dabigatran levels, whereas TG in the sample with low dabigatran levels is almost unaffected. The same trend was found in all 63 patients treated with dabigatran (Figure 6). The addition of idarucizumab causes a significant shortening of the lag time (median [IQR] without versus with idarucizumab: $4.7 \mathrm{~min}$ [3.3 $\mathrm{min}-7.0 \mathrm{~min}$ ] versus $2.7 \mathrm{~min}$ [2.3 $\mathrm{min}-3.3 \mathrm{~min}], \mathrm{p}<0.0001$ ) and the time-to-peak (7.8 min [5.6 min-9.7 min] versus 5.7 min [4.7 min-7.0 min], p<0.0001). One outlier can be seen with a small increase in lag time and time-to-peak after addition of idarucizumab (7.7 min to $8 \mathrm{~min}$ and $13.7 \mathrm{~min}$ to $14.7 \mathrm{~min}$, respectively). This patient took the last dabigatran tablet over $24 \mathrm{~h}$ before blood draw and was found to have $0 \mathrm{ng} / \mathrm{ml}$ dabigatran by HTI. The increase is therefore likely attributable to experimental variation. As expected, based on the results obtained in normal pooled plasma, no statistically significant difference was detected in peak height (median [IQR] with versus without idarucizumab: 241 nM [189 nM-310 $\mathrm{nM}$ ] versus $249 \mathrm{nM}$ [196 nM-280 nM], p = 0.30) and ETP (median [IQR] with versus without idarucizumab: 1,253 nM.min [1,054 nM.min-1,445 nM.min] versus 1,288 nM.min [1,120 
nM.min-1,511 nM.min], p = 0.18). However, a clear restoration of ETP and peak can be seen in the samples that were completely inhibited by dabigatran upon addition of idarucizumab. The velocity index is significantly reduced in the presence of idarucizumab (median [IQR]: $108 \mathrm{nM} / \min$ [61 nM/min-150 nM/min]) as compared to in the absence (median [IQR]: 86 $\mathrm{nM} / \min [56 \mathrm{nM} / \min -125 \mathrm{nM} / \min ])$
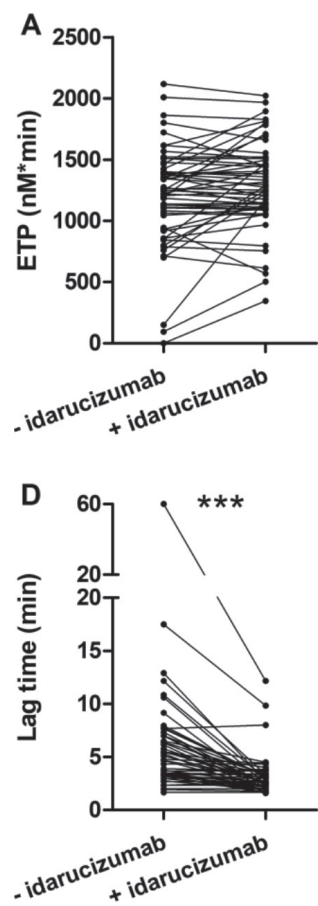
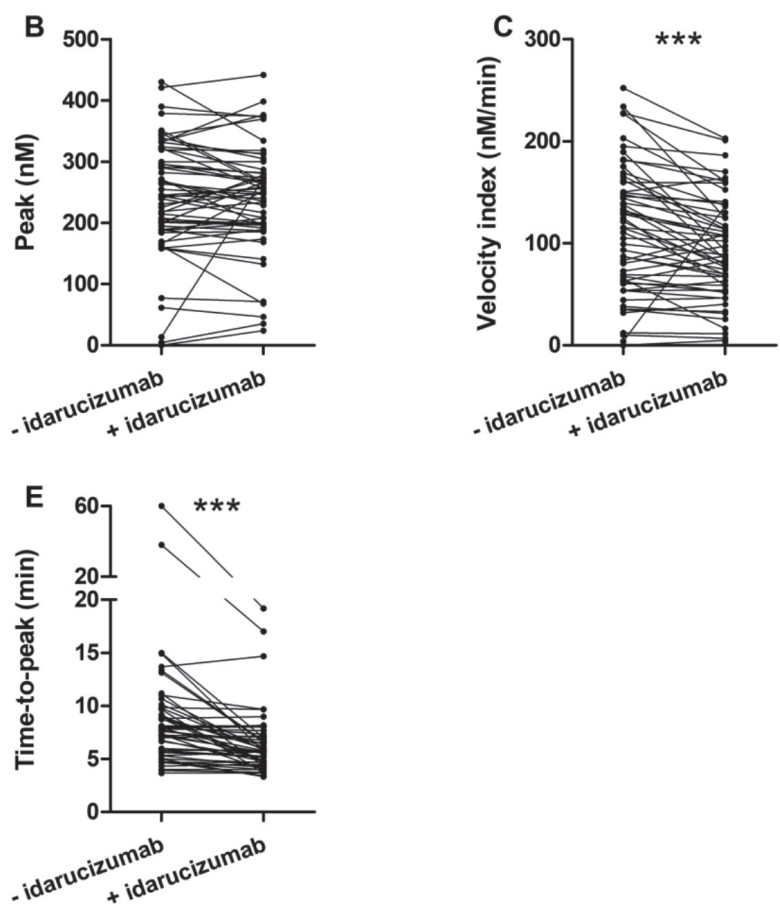

Figure 6 . The effect of idarucizumab on thrombin generation parameters in sixty-three patient samples containing dabigatran.

Endogenous thrombin potential (ETP) (A), peak (B), velocity index (C), lag time (D) and time-to-peak (E) before and after the in vitro addition of idarucizumab. Differences between groups were analyzed using the Wilcoxon signed rank test. ${ }^{* *} \mathrm{p}<0.0001, \mathrm{n}=63$ 


\section{DISCUSSION}

In this paper, we confirm the artefacts in TG that are observed in samples containing dabigatran (an increased peak and ETP), which were reported previously $24,26,27$; and we show that in CAT this increase in ETP and peak is caused by two mechanisms. Firstly, a large part is due to inhibition of the calibrator activity and a subsequent overestimation of thrombin activity in the TG measurement. We demonstrate that this effect can be overcome by the addition of dabigatran reversal agent idarucizumab to the calibrator measurement. Secondly, CAT uses a correction algorithm in which the fluorescence generated by a2M-thrombin activity is subtracted from the total thrombin activity (i.e. free thrombin plus a2M-thrombin). These calculations do not take into account that a2M-thrombin is inhibited by dabigatran to varying extents throughout the TG measurement and therefore TG is overestimated, especially at low dabigatran concentrations. ${ }^{27}$

In line with what was previously reported $24,26,27$, we found an elevation of TG in plasma containing dabigatran. Peak levels of 3,625 nM and ETP's of 17,301 nM.min were found. In normal populations analysed in the same conditions, but in the absence of anticoagulants, these values are on average $330 \mathrm{nM}$ and 1,555 nM.min, respectively. ${ }^{31}$ This elevation in peak and ETP was only partly restored when dabigatran in the calibrator well was either absent or neutralized. Since the velocity index is calculated by dividing the peak by the time between lag time and peak, this parameter is affected by the increased peak and therefore does not reflect the correct value either. For the neutralization of dabigatran idarucizumab was used, which was previously reported to completely reverse the dabigatran effect on TG.9,32,33 Dabigatran in the measurement well can also be completely neutralized by idarucizumab, which would allow assessment of TG as if no dabigatran was present ('uninhibited' conditions). However, the effect of dabigatran on the peak and ETP in CAT cannot be assessed this way. Under all circumstances the lag time and time-to-peak in CAT do correctly reflect the effect of dabigatran, since the time-dependent parameters are not altered by the corrections in the CAT algorithms.

Given that an elevation of the peak and/or ETP is still present after correcting the dabigatran effect on the calibrator, a second artefact must be situated in the correction for the a2M-thrombin in the TG well. A crucial assumption in the CAT algorithm is that the amount of a2M-thrombin increases over time as more thrombin is formed in pseudo first order 
reaction and consequently is proportional to the amount of thrombin. ${ }^{28}$ When dabigatran is present, the a2M-thrombin activity at the end of the measurement will be inhibited relatively more than at the beginning, since little thrombin will be left and a lot of a2M-thrombin is formed. This effect was more pronounced at $1 \mathrm{pM}$ TF as compared to $5 \mathrm{pM}$ TF. At lower TF concentrations, TG is known to be more sensitive and the ratio between free thrombin and a2M-thrombin will be affected differently compared to at $5 \mathrm{pM}$ TF. We speculate that when TG is initiated with 1 pM TF, the formation of thrombin proceeds slower compared to an initiation with $5 \mathrm{pM}$ TF and therefore relatively more a2M-thrombin is formed early in the process which is inhibited by dabigatran. At 20 pM TF the artefacts are not visible in the TG curves. However, a2M-thrombin is inhibited, resulting in an overestimation of the peak and ETP. Additionally, in the presence of higher dabigatran concentrations the peak is reduced compared to the absence of dabigatran. In these conditions the effect of dabigatran on free thrombin is larger than the effect on a2M-thrombin, therefore the net effect is a decreased, but still overestimated, TG.

The in vitro findings were corroborated in samples from patients containing a wide range of dabigatran concentrations (0-1,362 nM). Samples were analysed with idarucizumab present in the calibrator wells, and both in the presence and absence of idarucizumab in the TG wells. We additionally confirmed that idarucizumab does not affect TG in normal plasma. ${ }^{8,33}$ The results in the patient samples support the data in NPP spiked with dabigatran: addition of idarucizumab significantly decreased lag time as well as time-to-peak, yet the effect on ETP and peak was not significantly increased. The explanation for this is that about half of the collected samples contained low dabigatran concentrations. In these samples the counterintuitive decrease in peak and ETP after dabigatran neutralization could be seen. The velocity index is significantly reduced upon addition of idarucizumab. This parameter is partly based on time-dependent parameters and partly on the peak. Therefore, although this parameter is significantly altered in the before or after neutralization of dabigatran (due to the effect of the time-dependent factor), this parameter does not correctly reflect the extent of the anticoagulant effect of dabigatran.

Different solutions can be suggested to circumvent the artefacts in CAT calculations when dabigatran is present: (1) The measurement method can be altered in such a way that a2-M-thrombin is not detected in the experiment, or (2) the CAT algorithm can be updated to correctly perform the a2M-thrombin subtraction step in the TG calculation method. Firstly, 
to avoid the detection of a2M-thrombin activity in the TG measurement, a macrosubstrate could be used, which is only cleaved by free thrombin and not by a2M-thrombin. By using such a substrate the calculation artefacts would not occur. However, substrates with this quality that do not affect TG otherwise are currently not available. Secondly, in order to calculate the correct ETP and peak for samples containing both low and high concentrations of dabigatran, a new computational approach could be developed which allows the correct subtraction of a2M-thrombin activity. However, this activity varies between different patients since it is dependent on the a2M and antithrombin concentrations, as well as on the amount and the rate of thrombin generation. The additional complicating factor in patients using dabigatran is the fact that the interactions between these proteins are also influenced by the dabigatran levels. To correct for this an empirical computational approach would have to be developed to predict the amount of a2M-thrombin formed in individual patient samples with known a2M and dabigatran levels. However, the development of such an approach would require knowledge of the concentration of dabigatran present in the sample, the kinetic constants of the substrate as well as dabigatran with both thrombin and a2M-thrombin. Moreover, a complete correction of the artefacts would only be possible when implementing the computational approach together with the addition of idarucizumab to the calibrator. Validation of this algorithm would require devoted experimentation, calculations and moreover an evaluation in large and different patient populations. It would also require the measurement of plasma dabigatran levels in each plasma sample. Currently, we are working on an assay to measure dabigatran levels, which can be analysed in the same experimental set-up as TG accommodating the convenient and simultaneous measurement of dabigatran levels and the effect on coagulation.

The study had some limitations. Idarucizumab was used in a concentration of $6 \mu \mathrm{M}$, which can neutralize dabigatran concentrations up to $6 \mu \mathrm{M}(2,830 \mathrm{ng} / \mathrm{ml})$. The concentration of idarucizumab that was used, was high in comparison to findings by Jacquemin et al. who used $2.6 \mu \mathrm{M}$ idarucizumab to neutralize dabigatran in routine coagulation assays. ${ }^{34}$ The concentration used, on the other hand, was relatively low compared to patient plasma concentrations at the peak level after a $5 \mathrm{~g}$ dose (between $7 \mu \mathrm{M}$ and $45 \mu \mathrm{M}$ up to $30 \mathrm{~min}$ after infusion) [9]. However, for the purpose of demonstrating artefacts in CAT calculations in the presence of dabigatran, both in vitro and in peri-operative samples, this concentration was sufficient for full neutralization. 
Ideally, the TG peak and ETP would be used to evaluate the remaining haemostatic potential of a patient using dabigatran or the level of reversal achieved by the administration of idarucizumab. Using a thrombin generation test for this purpose would provide an insight into the general coagulation status of a patient. In this paper we show that the peak, ETP and velocity index can only be used to assess the 'uninhibited' coagulation potential by adding idarucizumab to the plasma. These parameters might, however, provide an idea of the anticoagulant effect of dabigatran at higher concentrations. Only the lag time and time-to-peak will give an accurate representation of the effect of dabigatran on CAT in all conditions, when idarucizumab is present in the calibrator well. Several studies have shown, though, that the time-dependent parameters tend to be less informative on the bleeding or thrombotic risk than the functional parameters (peak and ETP). ${ }^{35,36}$ Further research into possible solutions for the dabigatran effect on the CAT algorithms is required.

\section{ACKNOWLEDGEMENTS}

The authors would like to thank Rob Wagenvoord and Bas de Laat for their advice during the preparation of this article.

\section{DISCLOSURES}

S. Bloemen, R. Kremers, S. Zwaveling and M. Roest are linked to Synapse Research Institute, a research institute part of the Diagnostica Stago group. F. Mullier reports institutional fees from Stago, Werfen, Nodia, Sysmex and Bayer. He also reports speaker fees from Boehringer Ingelheim, Bayer Healthcare, Bristol-Myers Squibb-Pfizer and Stago all outside the submitted work. J. Douxfils declares not to have any conflict of interest. 


\section{REFERENCES}

1. European Medicines Agency, Summary for the Public: Pradaxa. . http://www.ema.europa.eu/docs/en_GB/ document_library/EPAR_-_Summary_for_the_public/human/000829/WC500041060.pdf.

2. Connolly SJ, Ezekowitz MD, Yusuf S, et al. Dabigatran versus warfarin in patients with atrial fibrillation. The New England journal of medicine. 2009;361(12):1139-1151.

3. Schulman S, Kearon C, Kakkar AK, et al. Extended use of dabigatran, warfarin, or placebo in venous thromboembolism. The New England journal of medicine. 2013;368(8):709-718.

4. Patel MR, Mahaffey KW, Garg J, et al. Rivaroxaban versus warfarin in nonvalvular atrial fibrillation. The New England journal of medicine. 2011;365(10):883-891.

5. Bauersachs R, Berkowitz SD, Brenner B, et al. Oral rivaroxaban for symptomatic venous thromboembolism. The New England journal of medicine. 2010;363(26):2499-2510.

6. Granger $\mathrm{CB}$, Alexander JH, McMurray JJ, et al. Apixaban versus warfarin in patients with atrial fibrillation. The New England journal of medicine. 2011;365(11):981-992.

7. Giugliano RP, Ruff CT, Braunwald E, et al. Edoxaban versus warfarin in patients with atrial fibrillation. The New England journal of medicine. 2013;369(22):2093-2104

8. Schiele F, van Ryn J, Canada K, et al. A specific antidote for dabigatran: functional and structural characterization. Blood. 2013;121(18):3554-3562.

9. Pollack CV, Jr., Reilly PA, Eikelboom J, et al. Idarucizumab for Dabigatran Reversal. The New England journal of medicine. 2015;373(6):511-520.

10. Vornicu O, Larock AS, Dincq AS, et al. Idarucizumab for the treatment of hemorrhage and dabigatran reversal in patients requiring urgent surgery or procedures. Expert opinion on biological therapy. 2017;17(10):1275-1296.

11. Tripodi A. The recommended dose of idarucizumab may not always be sufficient for sustained reversal of dabigatran: comment. Journal of thrombosis and haemostasis : JTH. 2017;15(9):1882-1883.

12. Simon A, Domanovits H, Ay C, Sengoelge G, Levy JH, Spiel AO. The recommended dose of idarucizumab may not always be sufficient for sustained reversal of dabigatran. Journal of thrombosis and haemostasis : JTH. 2017;15(7):1317-1321.

13. Douxfils J, Ageno W, Samama CM, et al. Laboratory testing in patients treated with direct oral anticoagulants: a practical guide for clinicians. Journal of thrombosis and haemostasis : JTH. 2018;16(2):209-219.

14. Neal MD, Levy JH. Precision Correction of Coagulopathy or Prothrombin Complex Concentrates?: Reversal Options for Dabigatran following Trauma. Anesthesiology. 2017;127(5):744-746.

15. Ten Cate H, Henskens YM, Lance MD. Practical guidance on the use of laboratory testing in the management of bleeding in patients receiving direct oral anticoagulants. Vascular health and risk management. 2017;13:457-467.

16. Dager WE, Gosselin RC, Kitchen S, Dwyre D. Dabigatran effects on the international normalized ratio, activated partial thromboplastin time, thrombin time, and fibrinogen: a multicenter, in vitro study. The Annals of pharmacotherapy. 2012;46(12):1627-1636.

17. Douxfils J, Dogne JM, Mullier F, et al. Comparison of calibrated dilute thrombin time and aPTT tests with LC-MS/ MS for the therapeutic monitoring of patients treated with dabigatran etexilate. Thrombosis and haemostasis. 2013;110(3):543-549.

18. Samuelson BT, Cuker A, Siegal DM, Crowther M, Garcia DA. Laboratory Assessment of the Anticoagulant Activity of Direct Oral Anticoagulants: A Systematic Review. Chest. 2017;151(1):127-138.

19. Douxfils J, Lessire S, Dincq AS, et al. Estimation of dabigatran plasma concentrations in the perioperative setting. An ex vivo study using dedicated coagulation assays. Thrombosis and haemostasis. 2015;113(4):862-869. 
20. Hemker HC, Giesen P, AIDieri R, et al. The calibrated automated thrombogram (CAT): a universal routine test for hyper- and hypocoagulability. Pathophysiology of haemostasis and thrombosis. 2002;32(5-6):249-253.

21. Al Dieri R, Peyvandi F, Santagostino E, et al. The thrombogram in rare inherited coagulation disorders: its relation to clinical bleeding. Thrombosis and haemostasis. 2002;88(4):576-582.

22. Dargaud $Y$, Beguin $S$, Lienhart $A$, et al. Evaluation of thrombin generating capacity in plasma from patients with haemophilia A and B. Thrombosis and haemostasis. 2005;93(3):475-480.

23. van Hylckama Vlieg A, Christiansen SC, Luddington R, Cannegieter SC, Rosendaal FR, Baglin TP. Elevated endogenous thrombin potential is associated with an increased risk of a first deep venous thrombosis but not with the risk of recurrence. $\mathrm{Br} \mathrm{J}$ Haematol. 2007;138(6):769-774.

24. Helin TA, Lemponen M, Hjemdahl P, Ronquist-Nii Y, Lassila R, Joutsi-Korhonen L. From laboratory to clinical practice: Dabigatran effects on thrombin generation and coagulation in patient samples. Thrombosis research. 2015;136(1):154-160

25. Giesen PLA, Gulpen AJW, van Oerle R, ten Cate H, Spronk HM. Calibrated Automated Thrombogram II: A new thrombin generation test able to measure in the presence of a direct thrombin inhibitor. Research and Practice in Thrombosis and Haemostasis 2017.

26. Gribkova IV, Lipets EN, Rekhtina IG, et al. The modification of the thrombin generation test for the clinical assessment of dabigatran etexilate efficiency. Scientific reports. 2016;6:29242.

27. Wagenvoord RJ, Deinum J, Elg M, Hemker HC. The paradoxical stimulation by a reversible thrombin inhibitor of thrombin generation in plasma measured with thrombinography is caused by alpha-macroglobulin-thrombin. Journal of thrombosis and haemostasis : JTH. 2010;8(6):1281-1289.

28. Hemker HC, Kremers R. Data management in thrombin generation. Thrombosis research. 2013;131(1):3-11.

29. Hemker HC, Giesen P, Al Dieri R, et al. Calibrated automated thrombin generation measurement in clotting plasma. Pathophysiology of haemostasis and thrombosis. 2003;33(1):4-15.

30. Coen Hemker H, Hemker PW, Al Dieri R. The technique of measuring thrombin generation with fluorescent substrates: 4. The H-transform, a mathematical procedure to obtain thrombin concentrations without external calibration. Thrombosis and haemostasis. 2009;101(1):171-177.

31. Bloemen S, Hemker HC, Al Dieri R. Large inter-individual variation of the pharmacodynamic effect of anticoagulant drugs on thrombin generation. Haematologica. 2013;98(4):549-554.

32. Pollack CV, Jr., Reilly PA, van Ryn J, et al. Idarucizumab for Dabigatran Reversal - Full Cohort Analysis. The New England journal of medicine. 2017;377(5):431-441.

33. Honickel M, Treutler S, van Ryn J, Tillmann S, Rossaint R, Grottke O. Reversal of dabigatran anticoagulation ex vivo: Porcine study comparing prothrombin complex concentrates and idarucizumab. Thrombosis and haemostasis. 2015;113(4):728-740.

34. Jacquemin $M$, Toelen $J$, Schoeters $J$, et al. The addition of idarucizumab to plasma samples containing dabigatran allows the use of routine coagulation assays for the diagnosis of hemostasis disorders. Journal of thrombosis and haemostasis : JTH. 2015;13(11):2087-2092.

35. Bosch YP, Al Dieri R, ten Cate $\mathrm{H}$, et al. Measurement of thrombin generation intra-operatively and its association with bleeding tendency after cardiac surgery. Thrombosis research. 2014;133(3):488-494

36. Bloemen S, Zwaveling S, Ten Cate H, Ten Cate-Hoek A, de Laat B. Prediction of bleeding risk in patients taking vitamin K antagonists using thrombin generation testing. PloS one. 2017;12(5):e0176967. 


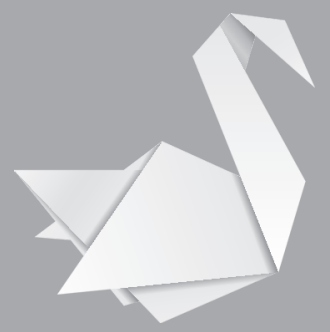




\section{CHAPTER}

A new assay for the concomitant assessment of rivaroxaban concentration and its impact on thrombin generation

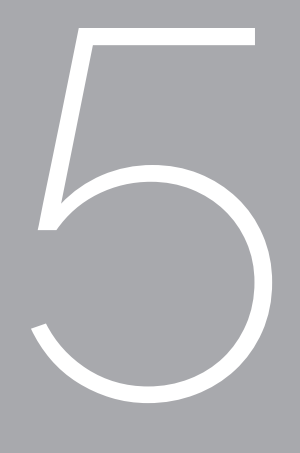

Saartje Bloemen* Suzanne Zwaveling* Jonathan Douxfils

Tessa Peters Rob Wagenvoord H. Coenraad Hemker

François Mullier Bas de Laat Mark Roest Romy Kremers

*These authors contributed equally to this work.

Submitted 


\section{ABSTRACT}

\section{Background}

Reliable assays to measure direct oral anticoagulant (DOAC) levels and their activity in critical situations are needed. Drug levels alone are not representative of the effect of DOACs on an individual's coagulation. We developed a technique that provides direct assessment of the global effect of rivaroxaban on the individual's coagulation in addition to the plasma concentration.

\section{Methods}

DOAC concentrations were determined in fifty patients using rivaroxaban, with the new assay, Xross-CAT. The effect of rivaroxaban on coagulation (activity) was measured with thrombin generation (TG) in platelet poor plasma using 5 pM tissue factor on the same device. The levels were validated with the Biophen DiXal assay. The prothrombin time (PT) and the dilute Russel viper venom (dRVV) confirm were performed to estimate the effect on coagulation.

\section{Results}

The total variability of Xross-CAT was below 12\%. Xross-CAT correlates well with Biophen DiXal $\left(r_{s=}\right.$ 0.885). The bias, determined by Bland-Altman analysis, was $4.9 \%$ and the Passing-Bablok equation was $y=1.1 x-2.1$. The correlation of plasma levels with TG was moderate (ETP $r_{s}=-0.548$; Peak $\left.r_{s}=-0.559\right)$, as for the PT $\left(r_{s}=0.739\right)$ and the DRVV confirm $\left(r_{s}=0.692\right)$.

\section{Conclusions}

Xross-CAT shows a good correlation with Biophen DiXal that was previously confirmed to accurately assess rivaroxaban levels. Bleeding and thrombotic complications are not necessarily associated with drug levels and could be influenced by concomitant risk factors. The main benefit of Xross-CAT is that it can provide an overview of the global anticoagulation status of a patient in relation to circulating DOAC levels. 


\section{INTRODUCTION}

Direct oral anticoagulants (DOACs) are currently prescribed according a fixed dose regimen in routine clinical practice. Their pharmacokinetic and pharmacodynamic profiles are more predictable and less prone to drug-drug interactions than those of VKAs. Therefore, DOACs are not monitored regularly. There are multiple clinical scenarios in which laboratory assessment of drug concentrations can be important (e.g. at the occurrence of a thrombotic or haemorrhagic event, before an invasive procedure, before thrombolysis therapy, in patients with a high bleeding risk, an extreme body weight, a decreased renal function, or when drug accumulation or an overdose is suspected). ${ }^{1-5}$ Furthermore, measuring DOACs may be helpful in assessing antidote requirement and around antidote administration ${ }^{2}$, to estimate the effect of drug-drug interactions or to guide the transition between VKAs and DOACs.

While drug levels can be useful to assess the individual's pharmacokinetic response, correlations to clinical outcome are still uncertain. Patients have developed thrombosis with DOAC levels in the "on-therapy" range. 6,7 Similarly, cases of spontaneous bleeding have been reported within the "on-therapy" range or even below this threshold, demonstrating that the drug level alone may not be sufficient to identify those who are more at risk. ${ }^{8,9}$ Additionally, there is a tendency to undertreat patients by choosing a lower dose of DOACs, out of fear of inducing bleeding complications. ${ }^{10-12}$ This scenario increases the risk of ischemic stroke without diminishing the risk is of bleeding, and should therefore be avoided by all means. Reliable tools to measure DOAC concentrations and their anticoagulant effect can help to prevent these situations and provide reassurance to the physician.

Rivaroxaban was the first direct factor Xa (FXa) inhibitor that was approved for clinical use. Assays to determine the rivaroxaban levels that are based on clotting times (e.g. prothrombin time (PT)) turned out to have limited sensitivity, high inter-reagent variability and poor comparability with specific assays for DOAC levels.,13-18 Standardized rivaroxaban-calibrated, chromogenic anti-FXa assays are currently the most reliable for the quantification of rivaroxaban levels in the routine laboratory. ${ }^{19}$ However, multiple studies have shown that drug levels in real-life patients can differ extremely between patients, 5,18,20,21 resulting in broad ranges of trough and peak levels $\left(6-87 \mathrm{ng} / \mathrm{ml}\right.$ at $\mathrm{T}_{\max }$ and from $189-419 \mathrm{ng} / \mathrm{ml}$ at $\mathrm{T}_{\text {trough }}$ in venous thromboembolism (VTE) patients, and $12-137 \mathrm{ng} / \mathrm{ml}$ and $184-343 \mathrm{ng} / \mathrm{ml}$ for atrial fibrillation 
(AF) patients, respectively). ${ }^{14}$ The variability of rivaroxaban levels within individuals has not yet been sufficiently investigated.

Besides this large inter-individual variation, meaningful interpretation of drug levels is complicated by a lack of guidance on extrapolating anti-Xa activity to bleeding and thrombosis. In other words, levels alone do not provide sufficient information about the influence of DOACs on the coagulation of individual patients. For that purpose, a functional test, which is able to measure both DOAC levels as well as their activity, is needed. The rivaroxaban test described in the current study, the Xross-CAT, is based on the capacity of rivaroxaban to inhibit both free FXa as well as bound FXa. Rivaroxaban can still bind to the active site of FXa when it is bound to $\mathrm{a}_{2}$-macroglobulin $\left(\mathrm{a}_{2} \mathrm{M}\right)$ or when it is in the prothrombinase complex, due to its small size and lack of necessity for a cofactor. ${ }^{22}$ It is known that direct thrombin inhibitors (e.g. dabigatran) interfere with the measurement of calibrated automated thrombinography (CAT) by inhibiting the calibrator ( $\mathrm{a}_{2} \mathrm{M}$-thrombin complex). ${ }^{23,24}$ We speculated that the $\mathrm{a}_{2} \mathrm{M}-\mathrm{FXa}$ complex will be inhibited by small molecule FXa inhibitors such as rivaroxaban. We used this principle to develop a test to measure plasma rivaroxaban levels. Since this test is based on the CAT method, it is possible to determine not only drug levels but also the overall coagulation activity (by determining thrombin generation) within the same setup. We hypothesize that this novel approach will provide an accurate measurement of DOAC concentrations together with activity in individual patients that provides a major advantage over currently used level assays.

\section{MATERIALS \& METHODS}

\section{Reagents}

Recombinant tissue factor (TF) was Innovin (Dade-Behring, Marburg, Germany). Synthetic phospholipids (PL) were purchased from Avanti Polar Lipids Inc. (Alabaster, AL, USA) and used in the form of vesicles consisting of phosphatidylserine, phosphatidylethanolamine and phosphatidylcholine (1:1:3, mol:mol:mol). The fluorogenic substrate Z-Gly-Gly-Arg aminomethylcoumarin (ZGGR-AMC) was obtained from Bachem (Basel, Switzerland). The calibrator ( $\mathrm{a}_{2} \mathrm{M}$-thrombin ( $\mathrm{a}_{2} \mathrm{M}$-Ila) $)$ was prepared according to Hemker et al. ${ }^{25}$ Hepes buffers containing $5 \mathrm{mg} / \mathrm{ml}$ or $60 \mathrm{mg} / \mathrm{ml}$ bovine serum albumin (BSA5 and BSA60) were prepared as described by Hemker et al. ${ }^{26}$ Rivaroxaban (Xarelto ${ }^{\circledR}$ ) was obtained by crushing tablets (Bayer, Berlin, Germany) or as powder (100\% purity) from Alsachim (Illkirch Graffenstaden, France). 


\section{Study population}

Fifty plasma samples from patients on rivaroxaban were collected from September 2014 until October 2015 in the CHU UCL Namur, Yvoir, Belgium. The study was performed in accordance with the Declaration of Helsinki, and sample collection was approved by the Medical Ethics Committee of the Centre Hospitalier Universitaire UCL Namur in Yvoir, Belgium (number of approval BU039201422406). The inclusion criteria were patients receiving rivaroxaban for secondary prevention of thromboembolic events in atrial fibrillation or treatment of venous thromboembolism, as well as collection of blood samples at trough (24 \pm 2 hours after last pill intake) or peak (2-3 hours after last pill intake) concentrations.

\section{Plasma preparation}

Blood was taken by venipuncture in the antecubital vein using a 21-gauge needle (Terumo) or through a peripheral venous catheter (BD Insyte-W, 18- or 16-gauge) and collected into 0.109 M sodium citrate (9:1 V/v) tubes (Venosafe, Terumo, Heverlee, Belgium). Platelet poor plasma (PPP) was obtained from the supernatant fraction after double centrifugation at 1,500 $\mathrm{g}$ for $15 \mathrm{~min}$ at room temperature. Samples were then aliquoted and frozen immediately at $-80^{\circ} \mathrm{C}$. Plasma sample aliquots were thawed and heated to $37^{\circ} \mathrm{C}$ for at least 5 min before running the experiment. Normal pooled plasma (NPP) was prepared from blood collected from 116 healthy volunteers. Blood was centrifuged at 2,500 g for 5 min after which plasma was pooled. The pooled plasma was then ultracentrifuged at 100,000 for $10 \mathrm{~min}$. Aliquots of $500 \mu \mathrm{l}$ were snap-frozen in liquid nitrogen and stored at $-80^{\circ} \mathrm{C}$ until analysis.

\section{Calibrated automated thrombinography}

Thrombin generation was performed in platelet poor plasma using CAT as described previously by Hemker et al. ${ }^{25}$ In summary, calibrator wells consisted of $20 \mu \mathrm{l}$ calibrator with 80 $\mu \mathrm{l}$ of plasma. Thrombin generation wells consisted of $20 \mu \mathrm{lTF} / \mathrm{PL}$ reagent and $80 \mu \mathrm{l}$ of PPP. The final concentration of TF was $5 \mathrm{pmol} / \mathrm{L}$ and $4 \mu \mathrm{mol} / \mathrm{L} \mathrm{PL}$. After $10 \mathrm{~min}$ of incubation at $37^{\circ} \mathrm{C} 20 \mu \mathrm{l}$ of $\mathrm{CaCl}_{2}$ solution containing the fluorescent substrate were dispensed into the wells. Data were analysed with specialized software from Thrombinoscope version 5.0 (Maastricht, The Netherlands). In each run, NPP was included in order to control for experimental variation. 


\section{a2M-FXa preparation}

The preparation of $\mathrm{a}_{2} \mathrm{M}-\mathrm{FX}$ a consists of two steps. Firstly, semi-pure $\mathrm{a}_{2} \mathrm{M}$ was prepared and in the second step the $a_{2} M$ was incubated with FXa to form the $a_{2} M-F X a$ complex. The $a_{2} M-F X a$ was purified further by running it on a Ni-Sepharose column. In step $1, a_{2} M$ was prepared by adding 3\% (w/v) PEG 20,000 to bovine plasma containing $10 \mathrm{mmol} / \mathrm{L}$ tri-sodium citrate and $4 \mathrm{mmol} / \mathrm{L}$ citric acid and incubating the suspension for $30 \mathrm{~min}$ at room temperature (RT) while stirring. The formed precipitate was spun down (25 min at $5277 \times \mathrm{g})$ and discarded. Then, an additional 3\% (w/v) PEG 20,000 was added to the supernatant containing $\mathrm{a}_{2} \mathrm{M}$ and again stirred for 30 min at RT. The precipitate was spun down as before. The pellet containing $\mathrm{a}_{2} \mathrm{M}$ was dissolved in $68.4 \mathrm{mmol} / \mathrm{L} \mathrm{NaCl}, 8.5 \mathrm{mmol} / \mathrm{L}$ Na-citrate ( $\mathrm{pH}$ 7.0). Residual fibrinogen was removed by adding $200 \mathrm{nmol} / \mathrm{L}$ thrombin, stirring for 20 min at RT and spinning down the formed fibrin at $5277 \times \mathrm{g}$ during $30 \mathrm{~min}$. The fibrin pellet was discarded. The traces of $\mathrm{a}_{2} \mathrm{M}$-thrombin and free thrombin in the supernatant were inactivated by addition of $400 \mathrm{nmol} / \mathrm{L}$ PPACK. The raw $\mathrm{a}_{2} \mathrm{M}$ was put at $4^{\circ} \mathrm{C}$ overnight in order to allow the excess PPACK to decay and then filtered $(0.22 \mu \mathrm{m})$ before further use.

For the preparation and purification of $\mathrm{a}_{2} \mathrm{M}-\mathrm{FXa} 8 \mathrm{ml} \mathrm{FXa}(70 \mu \mathrm{mol} / \mathrm{L})$ were added to 62 $\mathrm{ml} \mathrm{a} \mathrm{a}_{2} \mathrm{M}(10 \mu \mathrm{mol} / \mathrm{L})$ and incubated for $3.5 \mathrm{~h}$ at $37^{\circ} \mathrm{C}$. Twenty-three millilitres of $\mathrm{a}_{2} \mathrm{M}-\mathrm{FXa}$ were applied to a $150 \mathrm{ml} \mathrm{Ni-Sepharose} \mathrm{column}\left(5 \mathrm{~cm}^{2} \times 30 \mathrm{~cm}\right)$. Unbound material was removed by eluting the column with $350 \mathrm{ml} 500 \mathrm{mmol} / \mathrm{L} \mathrm{NaCl}, 20 \mathrm{mmol} / \mathrm{L}$ HEPES, $2 \mathrm{mmol} / \mathrm{L}$ imidazole, $0.02 \% \mathrm{NaN}_{3}\left(\mathrm{pH}\right.$ 7.35). Then the $\mathrm{a}_{2} \mathrm{M}-\mathrm{FXa}$ was eluted in a $750-\mathrm{ml}$ gradient of 2-32 $\mathrm{mmol} / \mathrm{L}$ imidazole and the obtained material was concentrated by precipitation in $40 \%$ saturated ammonium sulfate (= $242 \mathrm{~g} / \mathrm{L}$ ) by stirring for $30 \mathrm{~min}$ at RT and centrifuging for $20 \mathrm{~min}$ at $5277 \times \mathrm{g}$. The pellet was dissolved in calibrator buffer $(20 \mathrm{mmol} / \mathrm{L}$ HEPES, $100 \mathrm{mmol} / \mathrm{L} \mathrm{Na}{ }_{3}$-citrate, $0.02 \% \mathrm{NaN}_{3}(\mathrm{pH} 7.35)$ ) and centrifuged (20 min at $5277 \times \mathrm{g}$ ) to remove insoluble material before gel filtration. To the preparation $200 \mathrm{nmol} / \mathrm{L}$ AT and $2 \mathrm{U} /$ $\mathrm{ml}$ heparin were added to inactivate free FXa.

\section{DOAC concentration assay (Xross-CAT)}

The new Xross-CAT assay is based on the known CAT setup. Twenty microliters of $a_{2} \mathrm{M}-\mathrm{FXa}$ were incubated with $80 \mu \mathrm{l}$ of plasma diluted 8 times in Hepes buffer containing bovine serum albumin (BSA5: $5 \mathrm{mg} / \mathrm{ml} \mathrm{BSA}, 20 \mathrm{mmol} / \mathrm{L}$ Hepes, $140 \mathrm{mmol} / \mathrm{L} \mathrm{NaCl}$ and $0.02 \% \mathrm{NaN}_{3}$ at $\mathrm{pH}$ 7.35). The activity of rivaroxaban was gauged kinetically by the conversion of the fluorescent AMC-substrate on a Fluoroskan Ascent fluorimeter (Thermo Labsystems, Helsinki, 
Finland). Since it is usually unknown in what range the plasma concentration is situated, the samples had to be measured at two concentrations of $\mathrm{a}_{2} \mathrm{M}-\mathrm{Xa}$. The lower concentration was appropriate for determining levels between 22 and $218 \mathrm{ng} / \mathrm{ml}$ (which corresponds to 50-500 $\mathrm{nmol} / \mathrm{L}$ ) and the higher concentration for levels between 218 and $436 \mathrm{ng} / \mathrm{ml}$ (corresponding to 500-1000 nmol/L). These concentrations of $\mathrm{a}_{2} \mathrm{M}-\mathrm{X}$ a were selected based on an inhibition of the slope by rivaroxaban at different concentrations in the range of $15-85 \%$. The reference curves were prepared by spiking NPP with the required rivaroxaban concentrations to a constant volume BSA5 buffer. The samples were compared to two reference curves in each experiment $\left(22,44,87,131,174\right.$ and $218 \mathrm{ng} / \mathrm{ml}$ for the lower concentration of $\mathrm{a}_{2} \mathrm{M}$-Xa and $218,262,305,349,392$ and $436 \mathrm{ng} / \mathrm{ml}$ for the higher concentration). Fluorescence was measured during $15 \mathrm{~min}$. Using this method, up to 18 samples can be assessed in duplicate during each run in a total turnaround time of about 50 min, including double-centrifugation of the blood. The data of the samples were analysed by calculating the slope (0-10 min) of the fluorescent curve and comparing those values to the reference curves. Concentrations that were quantified below $44 \mathrm{ng} / \mathrm{ml}$ were not included in the analysis, as explained in the results section.

\section{PT Triniclot PT Excel $S \circledR$}

The prothrombin time was measured using Triniclot PT Excels S® (TrinityBiotech, Bray, Ireland) on a STA-R Evolution ${ }^{\circledR}$ coagulometer. Triniclot PT Excel S® is derived from rabbit brain. The PT was performed as previously described. ${ }^{27}$ We have chosen these reagents as they were known to be the more sensitive one. ${ }^{27}$

\section{Dilute russel viper venom time (DRVVT) confirm}

Briefly, $100 \mu \mathrm{l}$ of plasma sample was incubated during $240 \mathrm{~s}$ at $37^{\circ} \mathrm{C} .28$ Thereafter, $100 \mu \mathrm{l}$ of STA $\circledast$-Staclot ${ }^{\circ} D R V V$-Confirm (containing high PL concentration) was added, starting the reaction on a STA-R Evolution® coagulometer. Results are given in seconds.

\section{Biophen Direct Factor Xa Inhibitors}

Two hundred microliters of diluted plasma (1:50 with Tris-NaCl-EDTA buffer at $\mathrm{pH}$ 7.85) were incubated with $75 \mu \mathrm{l}$ of human FXa (Hyphen BioMed) for $120 \mathrm{~s}$ at $37^{\circ} \mathrm{C}$, then $75 \mu \mathrm{l}$ of a specific FXa substrate [CS-11(65)] (Hyphen BioMed) were added to start the reaction on a STA-R Evolution $®$ coagulometer (Diagnostica Stago). ${ }^{29}$ The concentrations of calibrators for the normal therapeutic range of rivaroxaban (Biophen Rivaroxaban Plasma Calibrator, 
Hyphen BioMed) were 50, 250 and $500 \mathrm{ng} / \mathrm{ml}$ in the initial samples after reconstitution. The low concentration procedure (Biophen DiXal LOW) was the same as the normal one except that plasma was diluted 1:8 in buffer and the calibration was performed with standards for low plasma concentrations of rivaroxaban (Biophen Rivaroxaban Calibrator Low). The standard rivaroxaban concentrations were 0,52 and $110 \mathrm{ng} / \mathrm{ml}$.

\section{Statistical analysis}

Statistical analyses were performed with Graphpad Prism software (version 5.00). Passing-Bablok regression was performed with MedCalc (version 17.7.2). Data are represented as mean with standard deviation (SD). Assay variation coefficients were calculated according to the Clinical and Laboratory Standards Institute (CLSI) guidelines. ${ }^{30}$ Normality of the data was assessed using the Shapiro-Wilk test. Correlation analysis was performed with the Spearman test. A two-sided p-value of 0.05 was considered statistically significant. Bland-Altman analysis was executed by analysing the percentage difference of the two methods $(100 *(A-B) / a v e r a g e)$ versus the average $((A+B) / 2)$ of the two methods. Method $A$ was the new rivaroxaban assay and method B was the Biophen DiXal assay. Passing-Bablok regression analysis was used to analyse the comparability of measurements, with the new assay on the $y$-axis and the established assay on the $x$-axis. The limit of detection (LOD) and limit of quantification (LOQ) were assessed according to ICH Q2R1 guideline. ${ }^{30}$ The LOD and LOQ were determined based on 5 measurements of the reference curve and 5 measurements of the blank as follows: LOD $=((3 * S D$ of $Y 0) /$ slope $)$ and LOQ $=((10 * S D$ of Y0)/slope); with YO being the y-axis intercept.

\section{RESULTS}

\section{Optimal a2M-FXa concentrations}

In order to set up this assay, the effect of different concentrations of rivaroxaban on $\mathrm{a}_{2} \mathrm{M}-\mathrm{FXa}$ was evaluated and found to be dose-dependent (Figure 1). Dose response curves were constructed to determine the concentrations of $\mathrm{a}_{2} \mathrm{M}-\mathrm{FX}$ a at which a linear relation with rivaroxaban concentrations was found. The curves were constructed at $10,20,30,40,50$, $60,70,80,90$ and $100 \mathrm{nmol} / \mathrm{L}$ of $\mathrm{a}_{2} \mathrm{M}-\mathrm{FXa}$, in the presence of eleven concentrations of rivaroxaban (between $22 \mathrm{ng} / \mathrm{ml}$ and $436 \mathrm{ng} / \mathrm{ml}$ ). The two $\mathrm{a}_{2} \mathrm{M}-\mathrm{FX}$ a concentrations were selected in which the inhibitory effects of all rivaroxaban concentrations were between 15 
and $85 \%$. Thirty $\mathrm{nmol} / \mathrm{L}$ of $\mathrm{a}_{2} \mathrm{M}-\mathrm{FX}$ a was the most fitting concentration for rivaroxaban levels from 22 to $218 \mathrm{ng} / \mathrm{ml}$ (Figure $2 \mathrm{~A}$ ) and $90 \mathrm{nmol} / \mathrm{L}$ of $\mathrm{a}_{2} \mathrm{M}-\mathrm{FX}$ a for levels between 218-436 $\mathrm{ng} / \mathrm{ml}$ (Figure 2B).

Table 1. Assay variability and accuracy.

\begin{tabular}{l|l|llllllll}
$\begin{array}{l}\text { Added } \\
\text { concentration } \\
(\mathrm{ng} / \mathrm{ml})\end{array}$ & $\begin{array}{l}\text { Mean } \\
\text { concentration } \\
(\mathrm{ng} / \mathrm{ml})\end{array}$ & SDwr & SDrr & SDdd & SDt & CVwr & CVrr & CVdd & CVt \\
\hline 43.6 & 51.0 & 5.1 & 1.1 & 0.2 & 5.3 & 10.0 & 2.2 & 0.4 & 10.4 \\
\hline 174.4 & 187.9 & 17.6 & 15.5 & 0.0 & 23.5 & 9.4 & 8.2 & 0.0 & 12.5 \\
\hline 436.0 & 464.3 & 16.2 & 23.4 & 24.5 & 37.5 & 3.5 & 5.0 & 5.3 & 8.1
\end{tabular}

SD, standard deviation; CV, coefficient of variation; wr, within run; rr, between run; dd, between day; t, total. SDs are presented in $\mathrm{ng} / \mathrm{ml}$. CVs are presented in \%.

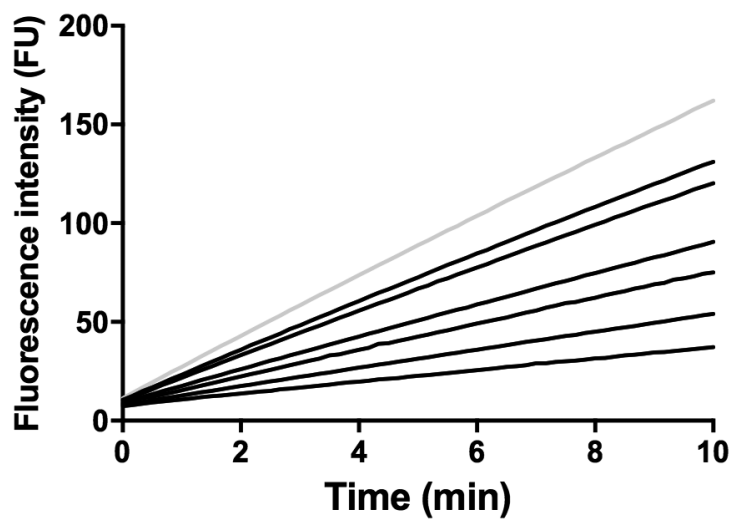

Figure 1. Dose dependent effect of different rivaroxaban concentrations on $a_{2} M-F X a$.

The effect of $0,22,44,87,131,174$ and $218 \mathrm{ng} / \mathrm{ml}$ (lines from top to bottom) of rivaroxaban (mean of two curves) on $\mathrm{a}_{2} \mathrm{M}-\mathrm{FX}$ a is illustrated. 

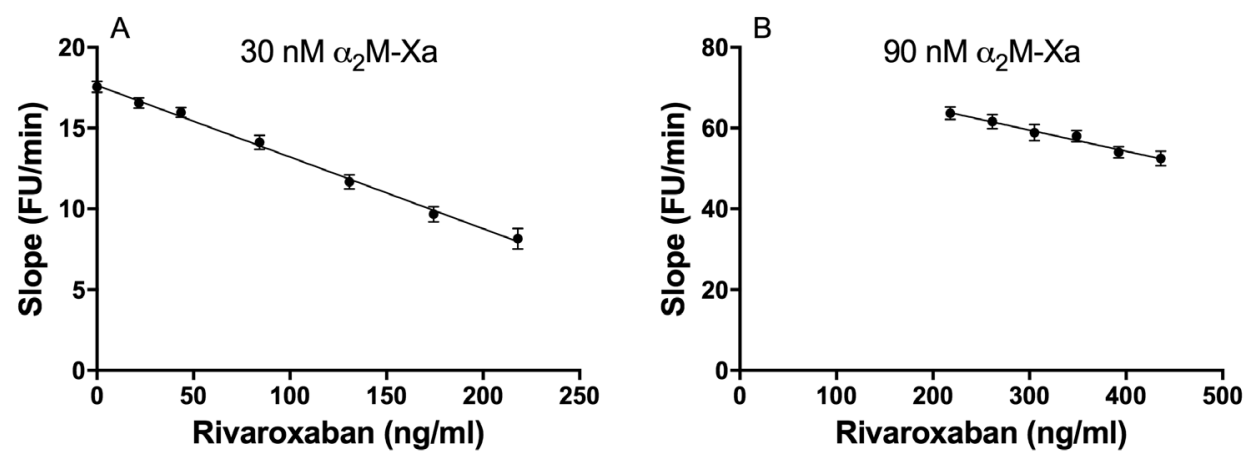

Figure 2. Dose-response of several rivaroxaban concentrations at chosen $a_{2} M-F X a$ concentrations.

Different concentrations of rivaroxaban measured at the $30 \mathrm{nmol} / \mathrm{L}(\mathrm{A})$ and $90 \mathrm{nmol} / \mathrm{L}(\mathrm{B}) \mathrm{a}_{2} \mathrm{M}-\mathrm{FXa} ; \mathrm{n}=5$. Data are presented as mean \pm SD.

\section{Assay performance}

The variability and accuracy of the assay are characterized in Table 1. These results suggest that the precision of the assay is adequate for the assay to be implicated in the laboratory. For the Xross-CAT the LOD was determined to be $3.3 \mathrm{ng} / \mathrm{ml}$ and the LOQ was $10.1 \mathrm{ng} / \mathrm{ml}$. However, the variability and accuracy of the assay for samples spiked with $22 \mathrm{ng} / \mathrm{ml}$, the coefficients of variation (CVs) increased considerably (a within-run variation of $37 \%$ and a between-run variation of $42 \%$ ). For all other tested concentrations $>22 \mathrm{ng} / \mathrm{ml}$ the $\mathrm{CVs}$ were below 13\% (Table 1). Since the CVs for $22 \mathrm{ng} / \mathrm{ml}$ were high, samples with concentrations below $<44 \mathrm{ng} / \mathrm{ml}$ were considered as unreliable and therefore excluded from analysis.

\section{Comparison of the assay to other tests}

In order to test the applicability of the new assay, it was compared to several assays that are known to be affected by rivaroxaban. Samples from 50 patients taking rivaroxaban were tested. The median time since last intake for the rivaroxaban samples was $3 \mathrm{~h}$ (min-max: 0-180h, interquartile range: 2-20h, $\mathrm{n}=44$ ).

We compared the Xross-CAT to the PT and the STA®-Staclot®DRVV-Confirm, which are both able to detect rivaroxaban but have limited sensitivity. The PT and DRVV confirm, displayed a relatively good correlation with our assay $\left(r_{s}=0.739\right.$ and 0.692 , respectively) (Figure 3). To evaluate the applicability of the new assays to detect concentration levels, we compared the Xross-CAT with the Biophen DiXal assay, which is currently used in the clinic as the most reliable assay to detect DOAC levels. Our assay showed the best correlation with the Biophen DiXal assay $\left(r_{s}=0.885\right)$ (Figure 4). As it can be expected that no addition 
of rivaroxaban would result in any detection by the Xross-CAT or the Biophen DiXal assay, the trendline was forced through zero. A Bland-Altman analysis was performed to analyse the agreement between our assay and the Biophen DiXal assay, which showed a bias of $4.9 \%$ between the two tests ( $95 \%$ limits of agreement: $-34.2 \%-44.0 \%$ ). The Passing-Bablok regression equation was $y=1.1(95 \% \mathrm{Cl}$ : 0.9 to 1.2$) x-2.1$ (95\% Cl: -41.8 to 27.8$)$.
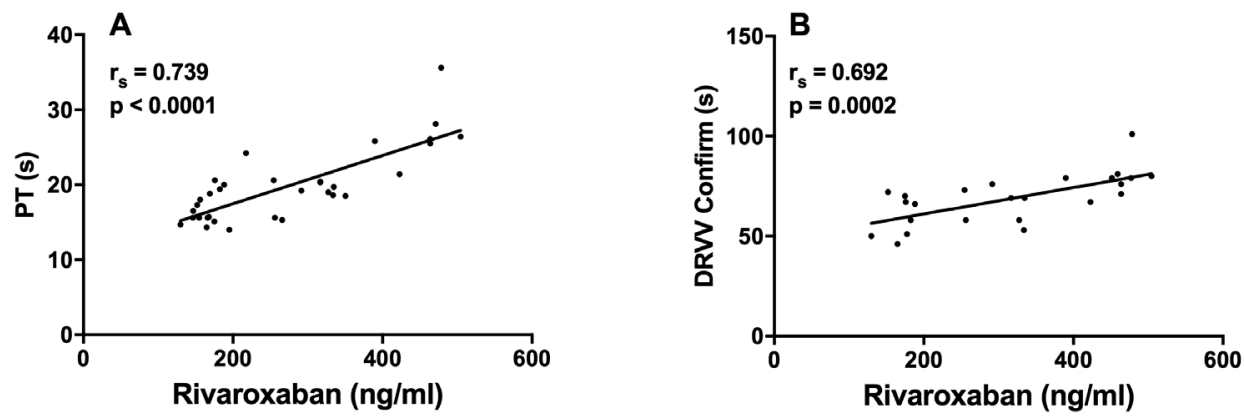

Figure 3. Correlation of rivaroxaban concentration (as determined by the Xross-CAT assay) with A) PT and B) dilute Russel Viper Venom time (DRVV) confirm.

The correlations were determined via Spearman correlation analysis. PT, $n=33$, DRVV confirm, $n=24$

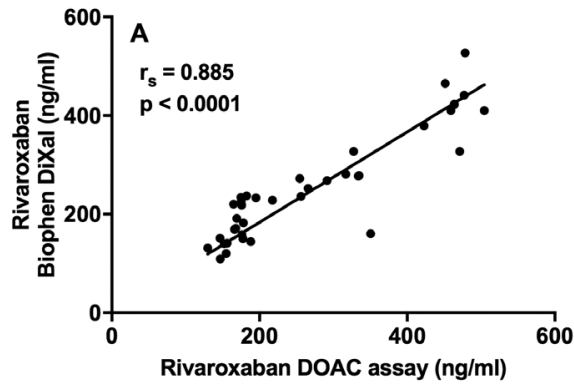

Figure 4. Correlation and Bland-Altman analysis between rivaroxaban concentration as determined by the XrossCAT assay and the concentration determined by the Biophen DiXal assay.

A) The correlations were determined via Spearman correlation analysis, $n=38$. B) The \% difference in the BlandAltman analysis is calculated as: $(100 *(A-B) / a v e r a g e)$ with $A$ being the concentration determined by the new rivaroxaban assay and $B$ the concentration determined by the Biophen DiXal assay, $n=38$. The mean difference was $4.9 \%$ (95\% limits of agreement: $-34.2 \%$ to $44.0 \%)$.

We also evaluated the correlation between the X-ross-CAT levels with the four main TG parameters: endogenous thrombin potential (ETP), peak, lag time and time-to-peak (ttPeak) (Figure 5). We found a moderate correlation between rivaroxaban levels and the lag time and time-to-peak $\left(r_{s}=0.646\right.$ and $r_{s}=0.601$, respectively). As anticipated, no linear correlation between the ETP and the peak was found. As is displayed in figure 5, there was an indication 
for a hyperbolic relation, which can be explained by the fact that low concentrations of rivaroxaban can already suppress the peak considerably. Above a certain concentration the peak is inhibited completely (a plateau phase is reached). This applies to the ETP as well.
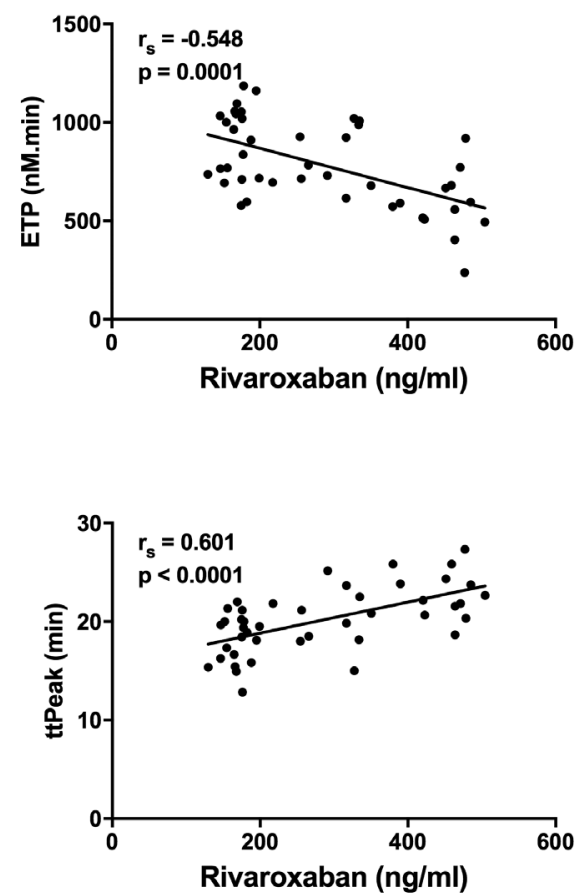
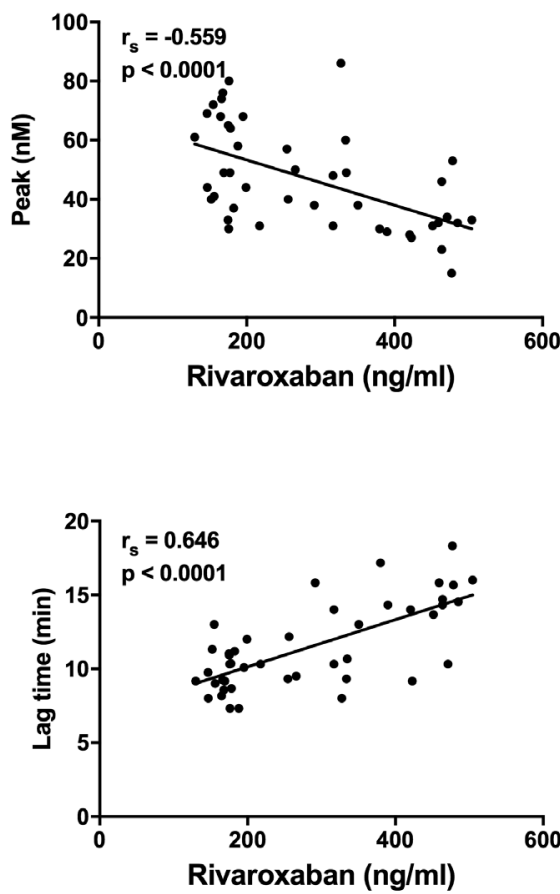

Figure 5. Correlation of rivaroxaban concentration and thrombin generation parameters.

The correlations of the rivaroxaban concentration, as determined by the Xross-CAT assay, with endogenous thrombin potential (ETP), peak, time-to-peak (ttPeak) and lag time were determined via Spearman correlation analysis, $n=44$.

\section{DISCUSSION}

In the past years, DOACs have become the primary choice as anticoagulants for patients with atrial fibrillation or venous thrombosis. One of the most important advantages of DOACs is that they can be given in a fixed dose and do not require regular monitoring. However, there are certain clinical scenarios in which laboratory assessment of DOAC levels is needed to optimize treatment and increase patient safety. As bleeding or thrombotic complications in patients receiving DOACs may not be systematically associated to the drug levels, a combined measurement of drug levels and global haemostatic activity might be more informative than drug levels alone. We developed an assay, the Xross-CAT, to measure 
plasma rivaroxaban levels via the dose-dependent inhibitory effect on the $\mathrm{a}_{2} \mathrm{M}$-Xa complex. We have optimized the test with a large range of rivaroxaban concentrations (44 to $436 \mathrm{ng}$ / $\mathrm{ml})$. These concentrations include in vivo rivaroxaban levels of patients with atrial fibrillation $(12-343 \mathrm{ng} / \mathrm{ml})$ and VTE $(6-419 \mathrm{ng} / \mathrm{ml}){ }^{4}$ Although not shown in the current manuscript, the principle of this test is also applicable to other direct FXa inhibitors (apixaban or edoxaban).

Many approaches have been proposed to measure rivaroxaban levels.,31,14,27 Some assays are based on clotting times, others on the anti-FXa activity. ${ }^{31}$ In order to specify which coagulation assays may be recommended for the measurement of rivaroxaban levels, they need to be compared to liquid chromatography coupled with tandem mass spectrometry (LC-MS/MS) or to the assays with the best relation to LC-MS/MS. At the moment, rivaroxaban-calibrated anti-FXa assays, such as the Biophen DiXal assay, showed the best correlations with LC-MS/MS. ${ }^{19,32}$ However, it should be taken into account that the quality of calibrators can differ. ${ }^{33}$ Xross-CAT showed a high agreement with the Biophen DiXal assay in the Bland-Altman and the Passing-Bablok analysis. This indicates that this newly developed assay reflects reliably the patient plasma concentrations. However, plasma levels assessed with the Xross-CAT are slightly higher compared to the Biophen DiXal assay, which can also be concluded from the Bland-Altman and the Passing-Bablok analysis. This is likely due to the use of different calibrators for the two assays. In the Xross-CAT, the use of homemade calibrators may introduce an unwanted bias. The use of the same sets of calibrators within the two assays could reduce this discrepancy. However, as samples in the Xross-CAT were measured retrospectively, this was not possible in the present study. Further validations are thus required to confirm this hypothesis. At this moment we were not able to reliably detect low concentrations of rivaroxaban $(22 \mathrm{ng} / \mathrm{ml})$ with the concentration of calibrator $\mathrm{a}_{2} \mathrm{M}-\mathrm{FXa}$ used, although this is not in agreement with the found LOD and LOQ of the assay. Therefore, we would not recommend using this assay to measure concentration lower than $44 \mathrm{ng} / \mathrm{ml}$.

The strength of our assay is that rivaroxaban concentrations can be measured in combination with the coagulation state of the patient, using thrombin generation. Our measurements displayed a significant correlation between rivaroxaban concentrations and thrombin generation parameters, which turned out to be the method of choice for measuring hyperand hypo-coagulable state. ${ }^{25,34-37}$ The correlations between rivaroxaban levels and ETP as well as peak describe a hyperbola. This can be explained because rivaroxaban inhibits the amplification phase of thrombin formation. Hence, the effect of rivaroxaban is more 
pronounced on ETP and peak. Moreover, this demonstrates that low levels of rivaroxaban are sufficient to reduce the amplification phase considerably. Therefore, at high rivaroxaban concentrations, the extent of the effect on peak and ETP is relatively small. ${ }^{38}$ Similar correlations were reported between rivaroxaban levels measured by chromogenic anti-FXa assays. ${ }^{39}$ It should be noted that in the large trials performed with DOACs ${ }^{40-42}$, drug levels were generally not compared to clinical outcomes, as the one-dose-fits-all principle was one of the important benefits of the new drugs. Ultimately, a large prospective clinical trial could solve the correlation between DOAC doses, levels and clinical outcomes. Because this would require a large study population and will be very costly, it is unlikely that such a trial will be performed in the nearby future. Therefore, the best option would be to study the relation between DOAC levels and the true effect of the DOACs on the coagulation system. Recently, an interesting observational study was published by Testa et al., which showed a relation between low trough levels of DOACs and the occurrence of thrombotic events in AF patients. Their results support the concept that measuring DOAC levels in a steady state could help identify low responders. ${ }^{7}$ This study was the first study of this sort, and only a relatively small number of patients were included. Larger studies are required for definite confirmation. Larger registries are currently on-going, and results are expected soon. Ideally, these studies will not solely test DOAC levels, but will also assess the effect of DOACs on coagulation.

For this purpose, a global coagulation test should be used with a good correlation to clinical outcomes. Multiple studies reported that thrombin generation is related to bleeding in patients treated with anticoagulants and in patients with heamophilia as well. ${ }^{35,43,44}$ Other studies have shown that thrombin generation parameters are related to thrombosis. ${ }^{45-47}$ Taking this into consideration, the main advantage of the new assay is that it would provide an evaluation of rivaroxaban concentrations and the effect of rivaroxaban on coagulation (via thrombin generation) within the same measurement.

There were also limitations to this study. The turn-around time of our assay is $50 \mathrm{~min}$, which is partly caused by the preparation of platelet poor plasma. The ideal DOAC test should allow near-patient measurements, to facilitate a direct decision regarding treatment. This would ideally be done in whole blood using a point-of-care device, but accurate near-patient tests are currently not available. Although measuring DOAC levels is becoming increasingly available, the importance for customizing treatment of individual patients remains to be 
delineated. Large clinical studies are required to establish the clinical value of those assays as well as for the definition of cut-off values. To further improve our test, an adaptation should be made, making it possible to accurately detect very low rivaroxaban levels, since it complicates the measurement of trough levels if these cannot be analysed.

In conclusion, the Xross-CAT shows a good correlation with a calibrated anti-FXa assay, which was confirmed to accurately assess rivaroxaban levels in patient samples. Measuring DOAC levels together with DOAC activity in an individuals' coagulation system provides an overview of the anticoagulation status in patients in relation to circulating DOAC levels. These findings could facilitate further developments towards personalized treatment in patients at risk of bleeding or recurrent thrombosis.

\section{DISCLOSURES}

Peters T, Wagenvoord R, de Laat B, Roest M and Kremers R are employed by Synapse Research Institute, which is part of Stago. Bloemen S and Zwaveling S are also linked to Synapse Research Institute. Bloemen S additionally reports personal fees from University Fund Limburg/ Foundation for Scientific Education Limburg (UFL/SWOL). Hemker HC reports personal fees from Stago. Mullier F reports institutional fees from Stago, Werfen, Nodia, Sysmex and Bayer. He also reports speaker fees from Boehringer Ingelheim, Bayer Healthcare, Bristol-Myers Squibb-Pfizer and Stago all outside the submitted work. Douxfils $J$ linked to QUALIblood sa and reports personal fees from Stago, Roche and Daiichi-Sankyo, outside the submitted work. 


\section{REFERENCES}

1. European Medicines Agency. Product Information: Xarelto. http://www.ema.europa.eu/docs/en_GB/document_library/EPAR_-_Product_Information/human/000944/WC500057108.pdf.

2. Levy JH, Ageno W, Chan NC, et al. When and how to use antidotes for the reversal of direct oral anticoagulants: guidance from the SSC of the ISTH. J Thromb Haemost. 2016;14(3):623-627.

3. Baglin T, Hillarp A, Tripodi A, Elalamy I, Buller H, Ageno W. Measuring Oral Direct Inhibitors (ODIs) of thrombin and factor Xa: A recommendation from the Subcommittee on Control of Anticoagulation of the Scientific and Standardisation Committee of the International Society on Thrombosis and Haemostasis. Journal of thrombosis and haemostasis : JTH. 2013.

4. Douxfils J, Ageno W, Samama CM, et al. Laboratory testing in patients treated with direct oral anticoagulants: a practical guide for clinicians. Journal of thrombosis and haemostasis : JTH. 2018;16(2):209-219.

5. Godier A, Dincq AS, Martin AC, et al. Predictors of pre-procedural concentrations of direct oral anticoagulants: a prospective multicentre study. European heart journal. 2017;38(31):2431-2439.

6. Ten Cate H, Olie RH, Ten Cate-Hoek AJ, Henskens YMC. Direct oral anticoagulants: When to consider laboratory testing? Int J Lab Hematol. 2018;40 Suppl 1:30-33.

7. Testa S, Paoletti O, Legnani C, et al. Low drug levels and thrombotic complications in high-risk atrial fibrillation patients treated with direct oral anticoagulants. Journal of thrombosis and haemostasis : JTH. 2018;16(5):842-848.

8. Albaladejo P, Bonhomme F, Blais N, et al. Management of direct oral anticoagulants in patients undergoing elective surgeries and invasive procedures: Updated guidelines from the French Working Group on Perioperative Hemostasis (GIHP) - September 2015. Anaesth Crit Care Pain Med. 2017;36(1):73-76

9. Sennesael AL, Larock AS, Douxfils J, et al. Rivaroxaban plasma levels in patients admitted for bleeding events: insights from a prospective study. Thromb J. 2018;16:28

10. Ogilvie IM, Newton N, Welner SA, Cowell W, Lip GY. Underuse of oral anticoagulants in atrial fibrillation: a systematic review. Am J Med. 2010;123(7):638-645 e634.

11. Nieuwlaat R, Capucci A, Lip GY, et al. Antithrombotic treatment in real-life atrial fibrillation patients: a report from the Euro Heart Survey on Atrial Fibrillation. Eur Heart J. 2006;27(24):3018-3026.

12. Wilke $T$, Groth A, Pfannkuche $M$, et al. Real life anticoagulation treatment of patients with atrial fibrillation in Germany: extent and causes of anticoagulant under-use. J Thromb Thrombolysis. 2015;40(1):97-107.

13. Kitchen S, Gray E, Mackie I, Baglin T, Makris M, committee B. Measurement of non-coumarin anticoagulants and their effects on tests of Haemostasis: Guidance from the British Committee for Standards in Haematology. Br J Haematol. 2014;166(6):830-841.

14. Samuelson BT, Cuker A. Measurement and reversal of the direct oral anticoagulants. Blood Rev. 2017;31(1):77-84.

15. Tripodi A. To measure or not to measure direct oral anticoagulants before surgery or invasive procedures. $J$ Thromb Haemost. 2016;14(7):1325-1327.

16. Avecilla ST, Ferrell C, Chandler WL, Reyes M. Plasma-diluted thrombin time to measure dabigatran concentrations during dabigatran etexilate therapy. Am J Clin Pathol. 2012;137(4):572-574.

17. Cuker A, Siegal DM, Crowther MA, Garcia DA. Laboratory measurement of the anticoagulant activity of the non-vitamin K oral anticoagulants. Journal of the American College of Cardiology. 2014;64(11):1128-1139.

18. Testa S, Tripodi A, Legnani C, et al. Plasma levels of direct oral anticoagulants in real life patients with atrial fibrillation: Results observed in four anticoagulation clinics. Thromb Res. 2016;137:178-183.

19. Douxfils J, Tamigniau A, Chatelain B, et al. Comparison of calibrated chromogenic anti-Xa assay and PT tests with LC-MS/MS for the therapeutic monitoring of patients treated with rivaroxaban. Thromb Haemost. 2013;110(4):723-731. 
20. Al-Aieshy F, Malmstrom RE, Antovic J, et al. Clinical evaluation of laboratory methods to monitor exposure of rivaroxaban at trough and peak in patients with atrial fibrillation. European journal of clinical pharmacology. 2016;72(6):671-679.

21. Samama MM, Martinoli JL, LeFlem L, et al. Assessment of laboratory assays to measure rivaroxaban--an oral, direct factor Xa inhibitor. Thromb Haemost. 2010;103(4):815-825.

22. Perzborn E, Kubitza D, Misselwitz F. Rivaroxaban. A novel, oral, direct factor Xa inhibitor in clinical development for the prevention and treatment of thromboembolic disorders. Hamostaseologie. 2007;27(4):282-289.

23. Wagenvoord RJ, Deinum J, Elg M, Hemker HC. The paradoxical stimulation by a reversible thrombin inhibitor of thrombin generation in plasma measured with thrombinography is caused by alpha-macroglobulin-thrombin. J Thromb Haemost. 2010;8(6):1281-1289.

24. Gribkova IV, Lipets EN, Rekhtina IG, et al. The modification of the thrombin generation test for the clinical assessment of dabigatran etexilate efficiency. Sci Rep. 2016;6:29242.

25. Hemker HC, Giesen P, Al Dieri R, et al. Calibrated automated thrombin generation measurement in clotting plasma. Pathophysiol Haemost Thromb. 2003;33(1):4-15.

26. Hemker HC, Hemker PW, Al Dieri R. The technique of measuring thrombin generation with fluorescent substrates: 4. The H-transform, a mathematical procedure to obtain thrombin concentrations without external calibration. Thromb Haemost. 2009;101(1):171-177.

27. Douxfils J, Mullier F, Loosen C, Chatelain C, Chatelain B, Dogne JM. Assessment of the impact of rivaroxaban on coagulation assays: laboratory recommendations for the monitoring of rivaroxaban and review of the literature. Thromb Res. 2012;130(6):956-966.

28. Douxfils J, Chatelain B, Hjemdahl P, et al. Does the Russell Viper Venom time test provide a rapid estimation of the intensity of oral anticoagulation? A cohort study. Thrombosis research. 2015;135(5):852-860.

29. Lessire S, Douxfils J, Pochet L, et al. Estimation of Rivaroxaban Plasma Concentrations in the Perioperative Setting in Patients With or Without Heparin Bridging. Clin Appl Thromb Hemost. 2016:1076029616675968.

30. Kennedy JW, Carey RN, Coolen RB, et al. Evaluation of Precision Performance of Clinical Chemistry Devices; Approved Guideline NCCLS. 1999;19(2).

31. van Pelt LJ, Lukens MV, Testa S, Chatelain B, Douxfils J, Mullier F. The DaXa-inhibition assay: A concept for a readily available, universal aXa assay that measures the direct inhibitory effect of all anti-Xa drugs. Thrombosis research. 2018;168:63-66.

32. Douxfils J, Gosselin RC. Laboratory Assessment of Direct Oral Anticoagulants. Semin Thromb Hemost. 2017;43(3):277-290.

33. Gosselin RC, Adcock D, Hawes EM, Francart SJ, Grant RP, Moll S. Evaluating the use of commercial drug-specific calibrators for determining PT and APTT reagent sensitivity to dabigatran and rivaroxaban. Thromb Haemost. 2015;113(1):77-84.

34. Al Dieri R, Peyvandi F, Santagostino E, et al. The thrombogram in rare inherited coagulation disorders: its relation to clinical bleeding. Thromb Haemost. 2002;88(4):576-582.

35. Dargaud $Y$, Beguin $S$, Lienhart $A$, et al. Evaluation of thrombin generating capacity in plasma from patients with haemophilia A and B. Thromb Haemost. 2005;93(3):475-480.

36. van Hylckama Vlieg A, Christiansen SC, Luddington R, Cannegieter SC, Rosendaal FR, Baglin TP. Elevated endogenous thrombin potential is associated with an increased risk of a first deep venous thrombosis but not with the risk of recurrence. Br J Haematol. 2007;138(6):769-774.

37. Al Dieri R, de Laat B, Hemker HC. Thrombin generation: what have we learned? Blood Rev. 2012;26(5):197-203.

38. Graff J, von Hentig N, Misselwitz F, et al. Effects of the oral, direct factor xa inhibitor rivaroxaban on platelet-induced thrombin generation and prothrombinase activity. J Clin Pharmacol. 2007;47(11):1398-1407. 
39. Artang R, Anderson M, Riley P, Nielsen JD. Assessment of the effect of direct oral anticoagulants dabigatran, rivaroxaban, and apixaban in healthy male volunteers using a thrombin generation assay. Research and Practice in Thrombosis and Haemostasis. 2017;1(2):194-201.

40. Connolly SJ, Ezekowitz MD, Yusuf S, et al. Dabigatran versus warfarin in patients with atrial fibrillation. N Engl J Med. 2009;361(12):1139-1151.

41. Patel MR, Mahaffey KW, Garg J, et al. Rivaroxaban versus warfarin in nonvalvular atrial fibrillation. N Engl J Med. 2011;365(10):883-891.

42. Giugliano RP, Ruff CT, Braunwald E, et al. Edoxaban versus warfarin in patients with atrial fibrillation. N Engl J Med. 2013;369(22):2093-2104.

43. Bosch $\mathrm{Y}, \mathrm{Al}$ Dieri $\mathrm{R}$, ten $\mathrm{Cate} \mathrm{H}$, et al. Preoperative thrombin generation is predictive for the risk of blood loss after cardiac surgery: a research article. J Cardiothorac Surg. 2013;8:154.

44. Dargaud $Y$, Lienhart A, Meunier S, et al. Major surgery in a severe haemophilia A patient with high titre inhibitor: use of the thrombin generation test in the therapeutic decision. Haemophilia. 2005;11(5):552-558.

45. Hron G, Kollars M, Binder BR, Eichinger S, Kyrle PA. Identification of patients at low risk for recurrent venous thromboembolism by measuring thrombin generation. Jama. 2006;296(4):397-402.

46. Tripodi A, Legnani C, Chantarangkul V, Cosmi B, Palareti G, Mannucci PM. High thrombin generation measured in the presence of thrombomodulin is associated with an increased risk of recurrent venous thromboembolism. Journal of thrombosis and haemostasis : JTH. 2008;6(8):1327-1333.

47. Tripodi A, Martinelli I, Chantarangkul V, Battaglioli T, Clerici M, Mannucci PM. The endogenous thrombin potential and the risk of venous thromboembolism. Thromb Res. 2007;121(3):353-359. 


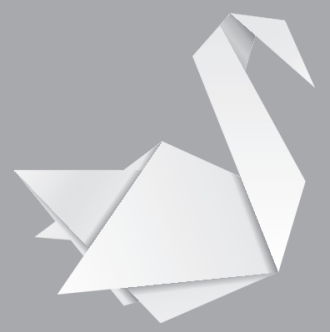




\section{CHAPTER}

The variability in response to rivaroxaban and apixaban between and within individuals, over time

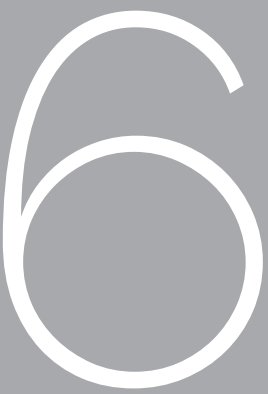

Suzanne Zwaveling Joke Konings

Hilde Kelchtermans

H. Coenraad Hemker

Saartje Bloemen

In preparation 


\section{ABSTRACT}

\section{Background}

Direct oral anticoagulants (DOACs) are prescribed at fixed dosages due to presumed stable pharmacokinetics and-dynamics. The relation between dose and plasma levels, as that between plasma levels and the effect on thrombin generation (TG), were found to be highly variable between persons. Here, the inter-individual response to rivaroxaban and apixaban was studied in addition to the stability within an individual over time.

\section{Methods}

The inter-individual variation was studied in platelet poor plasma (PPP) of 101 healthy donors. For the intra-individual response, blood of twelve healthy donors, was collected every two months to a total of six samples. TG was determined in PPP with or without addition of rivaroxaban or apixaban. The DOAC response was stated as \% inhibition of endogenous thrombin potential (ETP) or peak relative to the control. Additionally, prothrombin conversion and thrombin decay were studied.

\section{Results}

The variation of TG parameters between different individuals after addition of a fixed concentration of rivaroxaban or apixaban was high (CV up to 30.2\%). The inhibition of the ETP over time varied between $5.8 \%$ - 35.0\% for apixaban and $13.1 \%-41.2 \%$ for rivaroxaban; the peak between $2.7 \%-15.4 \%$, and $1.1 \%-6.2 \%$, respectively. Within individuals TG inhibition remained relatively stable over time, with some exceptions.

\section{Conclusions}

We confirmed that the in vitro response to a fixed DOAC concentration between persons is very variable. Here we show that the in vitro response of the same donor changes over time to a minor degree. In vivo the pharmacokinetic variation will likely superimpose on the pharmacodynamic variance detected here. 


\section{INTRODUCTION}

Direct oral anticoagulants (DOACs) specifically target thrombin (e.g. dabigatran) or factor Xa (e.g. rivaroxaban, apixaban, edoxaban). In large clinical trials it was shown that a fixed dosage without control of the anticoagulant effect is at least non-inferior to controlled use of vitamin $\mathrm{K}$ antagonists. ${ }^{1-6}$ The pharmacodynamic and -kinetic effects are stable $\mathrm{e}^{7-9}$ and therefore, guidelines do not recommend routine laboratory monitoring of DOACs, or any form of regular coagulation testing. Dose adjustments are only made on specific indications and patient characteristics (high age, extreme body weight, decreased renal and liver function and concomitant medication). Dose adjustment based on laboratory results, is not recommended. Only in exceptional cases measurement of DOAC levels can be considered (e.g. in patients who suffer from bleeding or thrombosis, prior to surgery or decreased renal clearance). ${ }^{10}$ However, there is a weak correlation between oral dose and plasma levels, which results in a broad "on therapy" range. ${ }^{11-14}$ Studies investigating DOAC levels in real life patients have reported a high variability between patients. ${ }^{15-20}$ The highest variation was seen in patients treated with the lowest dosage of DOACs ${ }^{16}$, which is the group of patients that is considered most vulnerable. Although the large variation in inter-individual levels is acknowledged, it is not considered a reason for laboratory testing and individual dose adjustment. The question remains whether these highly variable concentrations have an effect on overall coagulation efficiency and thereby possibly amplify the risk of thrombotic or bleeding events. There is some evidence that DOAC levels are associated with outcome events ${ }^{20-22}$, but the issue is complicated by the large variation over the day and between individuals. It has been found that patients with DOAC levels within the "on therapy" range can still develop major bleeding or thrombosis. ${ }^{23,24}$ These findings point out that DOAC levels do not necessarily present the effect of the DOAC on a patient's overall coagulation pattern.

Thrombin generation (TG) is a global coagulation assay, which has proven its ability to identify patients at risk of bleeding or thrombosis ${ }^{25-28}$ and which is able to detect the effect of many if not all anticoagulants. ${ }^{29-31}$ Therefore, the anticoagulant effect of DOACs on coagulation can indeed be assessed with TG. ${ }^{32-34}$ Our group previously found that the effect of DOACs (otamixaban and melagatran) on TG is highly variable between individuals, but these drugs are not in clinical use. Therefore, we have now assessed the inter-individual variability of rivaroxaban and apixaban. ${ }^{29}$ Furthermore, since DOACs are not being regularly monitored and no accurate tests are clinically available to measure their effect on coagulation, little or 
no data are available on the stability of their anticoagulant effect within individual donors over a longer period of time (i.e. several months). Only few studies have investigated the intra-individual stability of DOAC levels over a limited period of time and found a large variation in dabigatran levels (average CV 55\%) and lower CVs for rivaroxaban and apixaban levels (33\% and $19 \%$, respectively). ${ }^{16,17}$ In this study we set out to assess the effect of DOACs on TG in samples collected over the course of one year. To our knowledge, this study is the first to investigate the stability of the effect of rivaroxaban and apixaban on global coagulations within individuals over a longer time span.

\section{MATERIALS \& METHODS}

\section{Plasma samples}

Blood was collected from 101 healthy donors in Vacuette ${ }^{\circledR}$ tubes (Greiner Bio-One, Kremsmünster, Austria) containing 1 volume sodium citrate (3.2\%) to 9 volumes blood. Platelet poor plasma (PPP) was prepared by centrifuging the blood at 2,630 g for 10 min at room temperature, removing the supernatant and repeating the centrifugation. Aliquots were made of PPP and these were stored at $-80^{\circ} \mathrm{C}$ until use. Normal pooled plasma (NPP) was prepared by centrifuging blood from 116 healthy donors at 2,500 $\mathrm{g}$ for 5 min after which plasmas were pooled and ultracentrifuged at 10,000 g for $10 \mathrm{~min}$. Aliquots were snap frozen in liquid nitrogen and stored at $-80^{\circ} \mathrm{C}$. To assess the inter-individual variation in the DOAC effect blood of 101 donors was collected (one time point). To determine the within-subject variation, twelve of these donors were followed over a period of twelve months during which blood samples were collected each month. For this study six samples were analyzed (one sample every other month). All subjects provided informed consent.

\section{Reagents}

Innovin (Dade-Behring, Marburg, Germany) was used as a source of recombinant tissue factor (TF). Synthetic phospholipids (PL) were from Avanti Polar Lipids Inc. (Alabaster, AL, USA) and used in the form of vesicles consisting of phosphatidylserine, phosphatidylethanolamine and phosphatidylcholine (1:1:3, mol:mol:mol). Z-Gly-Gly-Arg-aminomethylcoumarin (ZGGR-AMC), the fluorogenic thrombin substrate, was obtained from Bachem (Basel, Switzerland). The calibrator (alpha-2-macroglobulin-thrombin ( $\mathrm{a}_{2} \mathrm{M}$-thrombin)) was prepared as previously described. ${ }^{35}$ Hepes buffers containing $5 \mathrm{mg} / \mathrm{ml}$ or $60 \mathrm{mg} / \mathrm{ml}$ bovine serum 
albumin (BSA5 and BSA60) were prepared according to the specifications by Hemker et al. ${ }^{36}$ Rivaroxaban (Xarelto ${ }^{\circledR}$ ) was obtained from Bayer (Berlin, Germany). Apixaban was purchased from Alsachim (Illkirch Graffenstaden, France).

\section{Calibrated automated thrombinography (CAT)}

Calibrated automated thrombinography (CAT) was performed using MidiCAT as previously described. ${ }^{37}$ Briefly, $10 \mu \mathrm{l}$ of calibrator were added to the calibrator wells in the presence of $40 \mu \mathrm{l}$ of PPP. In the TG wells $5 \mu \mathrm{l}$ of TF/PL solution were added to a well with either 5 $\mu$ of BSA5 buffer or $5 \mu$ of the rivaroxaban or apixaban solution after which $40 \mu$ of PPP were added to each well. NPP was used as a control for experimental variability in each experiment. Ten microliters of Z-Gly-Gly-Arg-aminomethylcoumarin (ZGGR-AMC) and 100 $\mathrm{mM} \mathrm{CaCl}_{2}$ in $\mathrm{BSA} 0$ buffer (FluCa) were dispensed to initiate the measurement. The final concentrations of the reagents were $5 \mathrm{pM} \mathrm{TF}, 4 \mu \mathrm{M} \mathrm{PL}, 16.7 \mathrm{mM} \mathrm{CaCl}_{2}, 416.7 \mu \mathrm{M}$ ZGGRAMC and $200 \mathrm{nM}(91.7 \mathrm{ng} / \mathrm{ml})$ apixaban. DOAC concentrations were chosen to be around $I_{50}$ for the endogenous thrombin potential (ETP) and peak, which was determined as the sum of the inhibition of the ETP and the peak and had to lie between $80-120 \% .{ }^{29}$ As (final) concentration of rivaroxaban for the experiments on the inter-individual variation between 101 donors, 400 nM (174.4 ng/ml) was used. For each measurement a NPP control was simultaneously measured, which allowed to correct for experimental variation. However, we noticed during the experiments that in NPP the effect of this concentration was quite high (ETP: $51 \%$, peak: 89\%). Therefore, not to obscure within-individual differences, we decreased the concentration to $300 \mathrm{nM}(130.8 \mathrm{ng} / \mathrm{ml})$ for this part of the study. For the assessment of the intra-individual variation, we controlled for the experimental variation by measuring all time points per donor on the same 96 wells plate.

\section{Calculation of prothrombin conversion and thrombin inactivation}

During a TG experiment, the net velocity of thrombin formation is always the result of the speed of prothrombin conversion and the speed of thrombin inhibition. We have calculated the course of prothrombin conversion from the measured net change in thrombin concentration and the constant course of thrombin inhibition (the decay constant) according to earlier specifications. ${ }^{38}$ During TG, thrombin is mainly inhibited by antithrombin (AT) and partly by $a_{2} M$. The thrombin decay capacity is a pseudo-first-order decay constant for thrombin that combines the overall effect of thrombin inactivation by AT and $\mathrm{a}_{2} \mathrm{M}$. The speed of thrombin inactivation can therefore be calculated from the measured level of thrombin and the decay 
constant. ${ }^{38}$ The prothrombin conversion curve is quantified by the area under the curve (AUC), which translates to the total amount of prothrombin converted throughout the TG experiment $\left(\mathrm{PC}_{\text {tot }}\right)$ and the peak height of the prothrombin conversion curve, which is the maximum prothrombin conversion rate (i.e. the maximum activity of the prothrombinase complex $\left.\left(P C_{\max }\right)\right)$. The calculations of these processes are referred to as thrombin dynamics.

\section{Data analysis}

The effect of the DOACs was expressed as percentage inhibition of ETP or peak or as prolongation of the lag time of time-to-peak. ${ }^{29}$ Coefficients of variation were the standard deviation expressed as a percentage of the mean ( $C V=100 * S D / m e a n)$. The total $C V\left(C V_{\text {total }}\right)$ is a composite of experimental error $\left(\mathrm{CV}_{\text {exp }}\right)$ and the inter-individual variability $\left(\mathrm{CV}_{\mathrm{ii}}\right)$. The $\mathrm{CV}_{\text {exp }}$ was calculated from the data from NPP, which was included in each run. The $\mathrm{CV}_{\mathrm{ii}}$ was then calculated as $C V_{i i}=\sqrt{C V_{\text {total }}^{2}-C V_{\text {exp }}^{2}}$. The within-subject $C V$ s were calculated per individual donor from the inhibition of the TG-parameters over the six time points.

\section{Statistical analysis}

Statistical analyses were performed using GraphPad Prism version 5.00 (GraphPad Software Inc., La Jolla, CA, USA). Correlations between thrombin dynamics and TG parameters were calculated using the Spearman correlation test. Results were considered statistically significant at $p<0.05$.

\section{RESULTS}

\section{Inter-individual variation}

The inter-individual variation in TG parameters showed a CV of 13.0\% - 15.4\% in the absence of a DOAC (Table 1, upper left part). After the addition of a fixed concentration of rivaroxaban or apixaban the CV's increased to $12.6 \%-44.9 \%$ and $13.5 \%-35.8 \%$, respectively (Table 1, lower left part). This increase is related to the high variability in the inhibitory effects of DOACs on individual plasmas, i.e. 3.2\% - 29.0\% for rivaroxaban and $6.0 \%-44.5 \%$ for apixaban (Table 1, right part). The increase in variability is mostly shown in the peak: an increase of $15.4 \%$ in the absence of DOACs, compared to $44.9 \%$ or $35.8 \%$ in the presence of rivaroxaban or apixaban, respectively. The presence of direct factor Xa inhibitors causes a particular shape change of the TG curve, characterized by a more protracted curve with a 
relatively low peak (Figure 1). Accordingly, the inhibiting effect on the peak was much more pronounced (an average inhibition of $79 \%$ for rivaroxaban and $64 \%$ for apixaban on $\mathrm{T} 1$ ) than the suppressing effect of direct factor Xa inhibitors on the ETP (an average inhibition of 20\% and $16 \%$, respectively). The CV's of time-dependent parameters as lag time and time-to-peak remain stable after addition of rivaroxaban and apixaban.

Table 1. Inter-individual variation of TG parameters and inhibition induced by rivaroxaban and apixaban.

\begin{tabular}{|c|c|c|c|c|c|c|c|c|c|c|c|}
\hline & & Meası & ed va & & & & Inhibit & ry effe & & & \\
\hline Inter-individual vari & ation & Mean & SD & $\begin{array}{l}\mathrm{CV}_{\text {total }} \\
(\%)\end{array}$ & $\begin{array}{l}\mathrm{CV}_{\text {exp }} \\
(\%)\end{array}$ & $\begin{array}{l}\mathrm{CV}_{\mathrm{ii}} \\
(\%)\end{array}$ & Mean & SD & $\begin{array}{l}\mathrm{CV}_{\text {total }} \\
(\%)\end{array}$ & $\begin{array}{l}\mathrm{CV}_{\text {exp }} \\
(\%)\end{array}$ & $\begin{array}{l}\mathrm{CV}_{\mathrm{ii}} \\
(\%)\end{array}$ \\
\hline & Lag time & 2.1 & 0.4 & 17.5 & 8.6 & 15.2 & - & - & - & - & - \\
\hline $5 n M T-n-101$ & ETP & 1093 & 165 & 15.1 & 3.6 & 14.7 & - & - & - & - & - \\
\hline 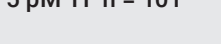 & Peak & 272 & 43 & 15.7 & 3.1 & 15.4 & - & - & - & - & - \\
\hline & ttPeak & 4.2 & 0.6 & 14.1 & 5.4 & 13.0 & - & - & - & - & - \\
\hline & Lag time & 6.3 & 1.0 & 16.2 & 6.8 & 14.7 & 3.1 & 0.3 & 10.3 & 7.6 & 6.9 \\
\hline $5 \mathrm{pM} T F+400 \mathrm{nM}$ & ETP & 661 & 186 & 28.1 & 6.5 & 27.3 & 42.6 & 12.5 & 29.3 & 4.6 & 29.0 \\
\hline rivaroxaban $\mathrm{n}=77$ & Peak & 35 & 16 & 45.5 & 7.2 & 44.9 & 89.2 & 3.0 & 3.4 & 0.8 & 3.2 \\
\hline & ttPeak & 16.7 & 2.2 & 13.3 & 4.1 & 12.6 & 4.0 & 0.6 & 13.7 & 4.3 & 13.1 \\
\hline & Lag time & 3.5 & 0.6 & 16.7 & 8.8 & 14.2 & 1.7 & 0.1 & 8.8 & 6.4 & 6.0 \\
\hline $5 \mathrm{pM} \mathrm{TF}+200 \mathrm{nM}$ & ETP & 974 & 144 & 14.8 & 6.2 & 13.5 & 10.6 & 5.0 & 47.6 & 16.9 & 44.5 \\
\hline apixaban n = 101 & Peak & 104 & 38 & 36.3 & 5.8 & 35.8 & 63.8 & 8.4 & 13.2 & 1.8 & 13.1 \\
\hline & ttPeak & 6.9 & 1.0 & 14.5 & 5.1 & 13.5 & 1.7 & 0.1 & 7.4 & 4.2 & 6.1 \\
\hline
\end{tabular}

n, number of subjects; TF, tissue factor; ETP, endogenous thrombin potential; ttPeak, time-to-peak; SD, standard deviation; $C V$, coefficient of variation; $\mathrm{CV}_{\text {total }}$, total variation; $\mathrm{CV}_{\text {exp }}$ experimental variation; $\mathrm{CV}_{\mathrm{i}}$, inter-individual coefficient of variation

The units of the measured values are: lag time, min; ETP; nM. min; Peak, nM; ttPeak, min. For the effect the following values are noted: lag time and ttPeak, prolongation compared to without anticoagulant; ETP and peak, inhibition (\%) compared to without anticoagulant. 

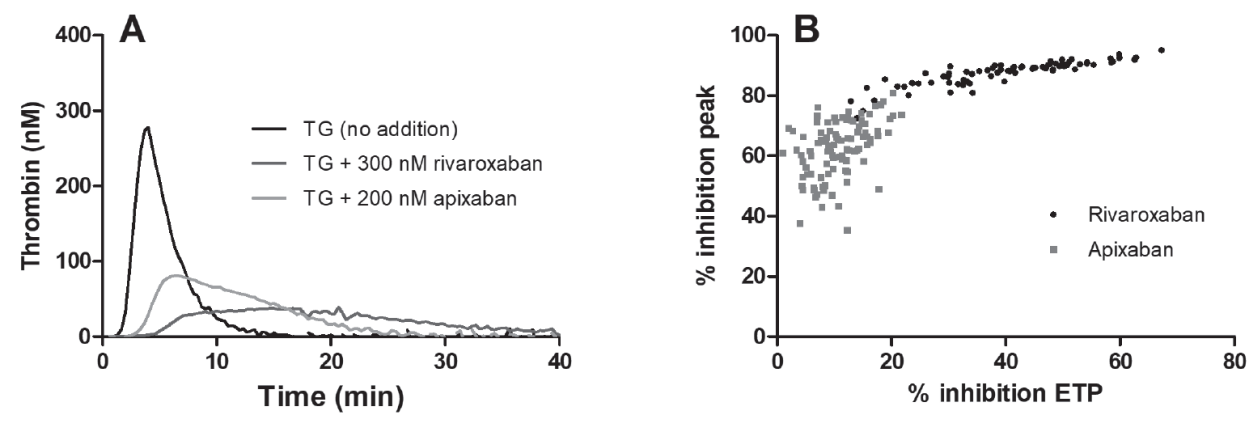

Figure 1. The effect of rivaroxaban and apixaban on thrombin generation (TG).

(A) Upon addition of a fixed concentration of rivaroxaban (300 nM) or apixaban ( $200 \mathrm{nM})$ a protracted TG curve can be seen (exemplary curves). (B) The \% inhibition of the peak versus the endogenous thrombin potential (ETP) for the addition of rivaroxaban $(400 \mathrm{nM})$ or apixaban $(200 \mathrm{nM})$ to the plasmas of healthy individuals $(n=77, n=101$, respectively).

\section{Intra-individual variation}

The variation in TG after the addition of DOACs measured with TG within individuals over time is shown in figure 2. The ETP and peak in the absence of DOACs remain reasonably stable over time (Figure 2A and B) (CV of 3.4\% - 14.2\% for the ETP and 1.2\%-8.5\% for the peak). When a fixed dose of rivaroxaban or apixaban was added the variability in remaining TG increases, particularly for the peak (CV 5.1\% - 25.2\% for rivaroxaban and 10.3\% - 25.7\% for apixaban) (Figure 2D and F and Table 2). For the ETP the CV's ranged from $3.1 \%-16.1 \%$ for rivaroxaban, and from 3.5\% - 11.8\% for apixaban (Figure 2C and E and Table 2). The time-dependent variables remain more stable, as shown table 2. 
A No addition

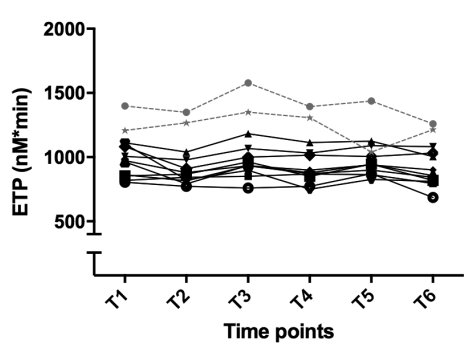

C Rivaroxaban
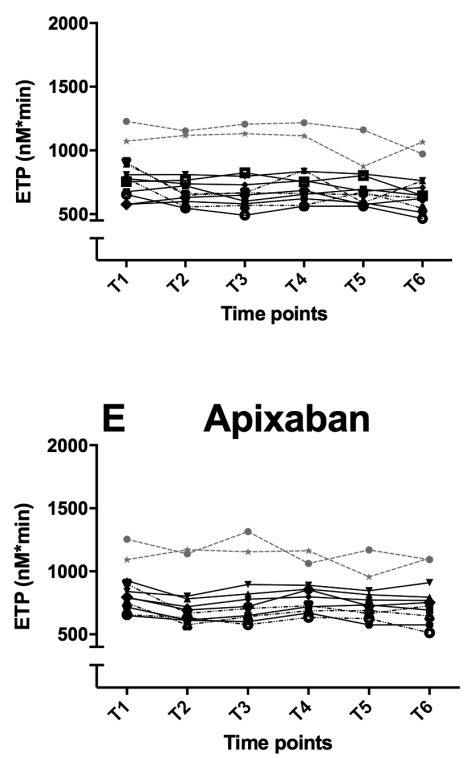

B No addition

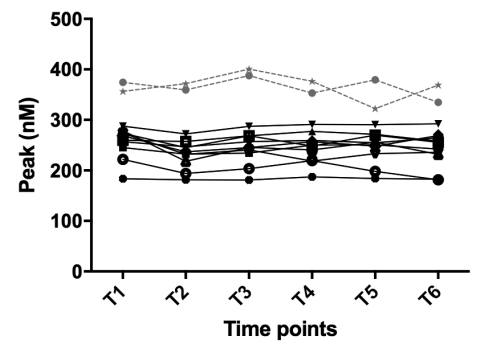

D Rivaroxaban
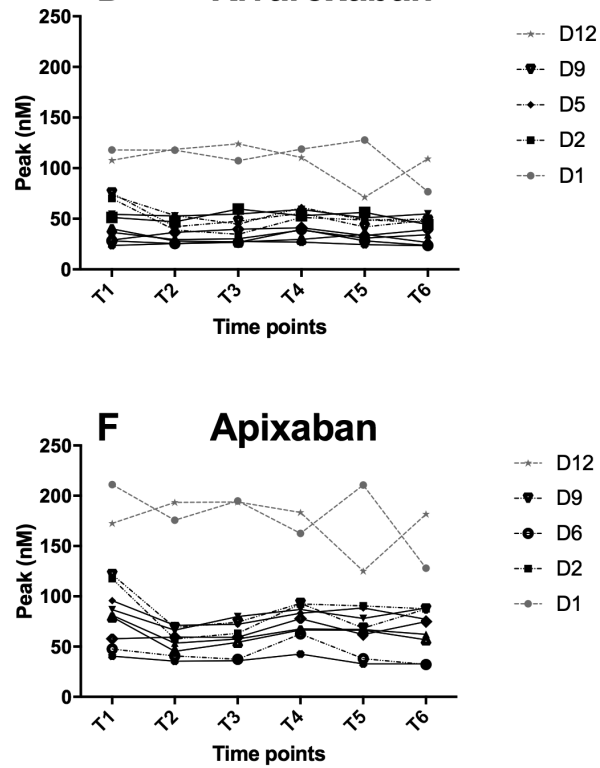

Figure 2. The endogenous thrombin potential (ETP) and peak of twelve individual donors before and after the addition of fixed concentrations rivaroxaban and apixaban.

The residual ETP and peak in the absence of anticoagulants (A-B) and after adding a fixed dose rivaroxaban (300 $\mathrm{nM})(\mathrm{C}-\mathrm{D})$ or apixaban (200 nM) (E-F) in plasma of healthy donors followed over one year. Individuals with the highest variability over time, i.e. with CVs in the upper quartile, are indicated with a black dotted line. The gray dotted line displays the two individuals using hormonal contraceptives. 


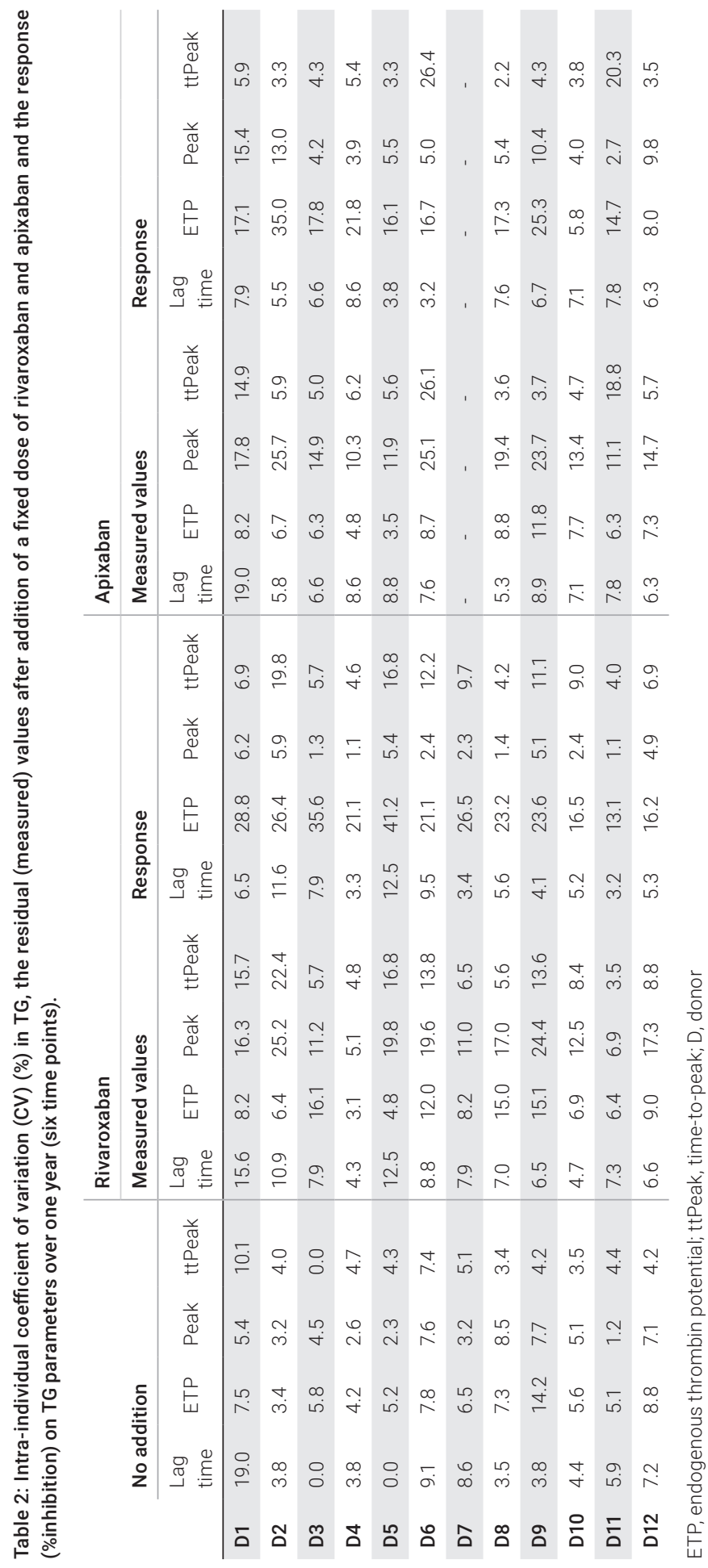


An increase in variability in the response to rivaroxaban and apixaban within individuals is particularly visible for the effect on the ETP (Figure 3 and Table 2). Generally, the remaining ETP and peak as well as the response of these parameters after addition of DOACs were quite stable over time, but there were some exceptions. As can be observed from figure 3C, the ETP of donor 3 was inhibited almost three times as much at T9 compared to T1, in response to a fixed concentration of rivaroxaban.

When studying the course of TG (Figure 2) two individuals (D1 and D12) have a markedly higher TG, in the presence and absence of the DOACs, than the other donors. Interestingly, these were the only two donors that were using a form of hormonal contraception (one a $3^{\text {rd }}$ generation oral contraceptive and one a vaginal ring). To compare the variation in donors over time between rivaroxaban and apixaban, we calculated the upper quartiles for the variability in the remaining ETP and peak as well as for the response. The donors with the highest variability over time are indicated in figures 2 and 3. Interestingly, the individuals that were least stable on rivaroxaban were not necessarily the same as the ones that were the least stable on apixaban.
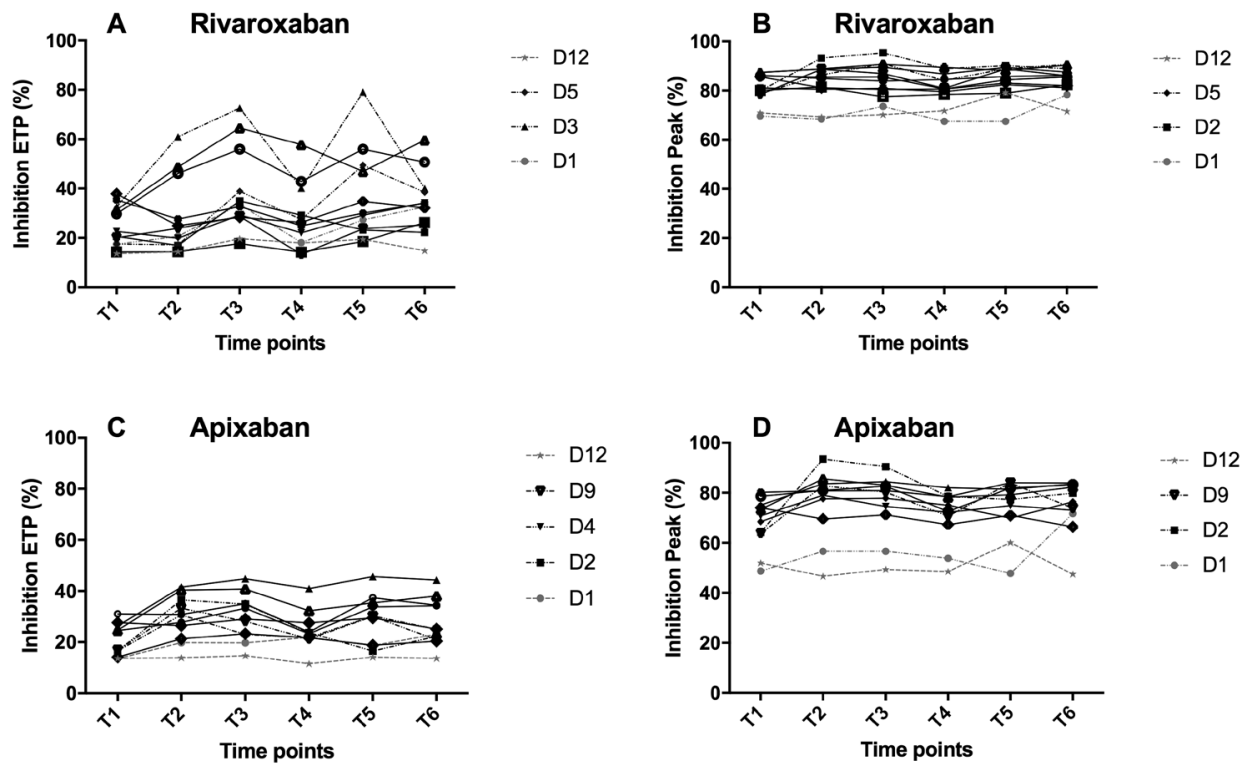

Figure 3. Response of twelve individual donors to rivaroxaban and apixaban.

The percentage inhibition of the endogenous thrombin potential (ETP) and peak in response to a fixed dose of rivaroxaban (300 nM) (A-B) and apixaban (200 nM) (C-D) in the plasma of healthy donors followed over one year. Individuals with the highest variability over time, i.e. with $\mathrm{CV}$ s in the upper quartile, are indicated with a dotted black line. The gray dotted line indicates the two individuals using hormonal contraceptives. 

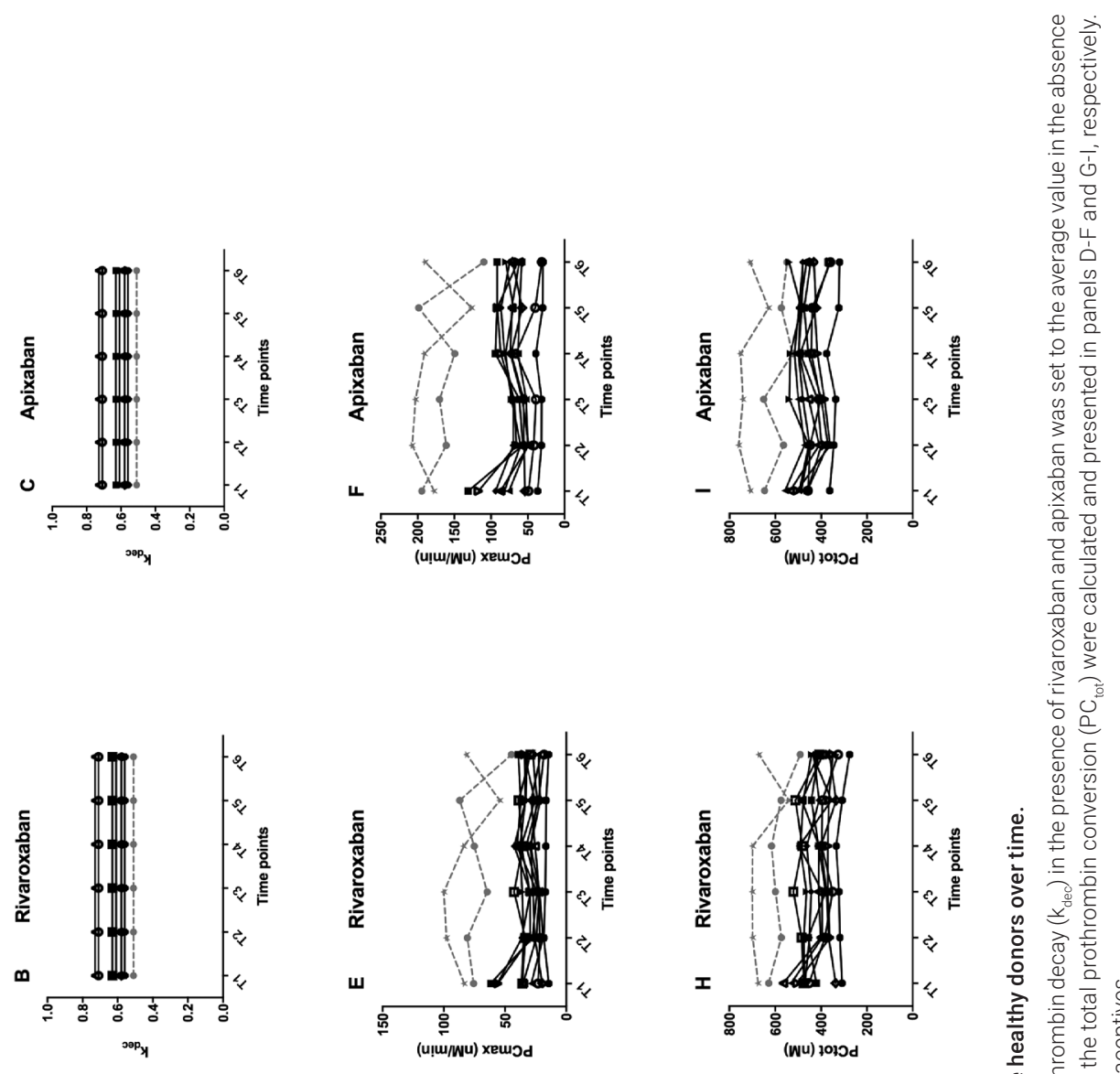
The prothrombin conversion and thrombin decay over time are plotted in figure 4 . The rate of thrombin decay shows good stability over time in the absence of the direct factor Xa inhibitors. Since the decay (AT and $a_{2} M$ ) is not affected by direct $X a$ inhibitors and could not be properly calculated due to the particular shape of TG curves under these conditions, the $\mathrm{k}_{\mathrm{dec}}$ was set to the average value found in the absence of DOACs. The $\mathrm{PC}_{\max }$ and the $\mathrm{PC}_{\text {tot }}$ were also relatively stable over time, and followed the same pattern as the ETP and peak of TG. In the absence of DOAC the $r_{s}$ of the $P C_{\max }$ was 0.565 and 0.897 with the ETP and the peak, respectively $(p<0.001)$. For the PC $_{\text {tot }}$ the $r_{s}$ was 0.725 with the ETP and 0.697 with the peak $(p<0.001)$. When rivaroxaban or apixaban was added, the $r_{s}$ of the $P_{\text {max }}$ with the ETP was 0.824 (rivaroxaban) and 0.761 (apixaban) and with the peak 0.973 (rivaroxaban) and 0.969 (apixaban) $(p<0.001)$. In case of the $P_{\text {tot }}$ the $r_{s}$ with the ETP was 0.899 (rivaroxaban) and 0.885 (apixaban), and with the peak 0.786 (rivaroxaban) and 0.763 (apixaban) $(p<0.001)$. In all three conditions the $k_{\text {dec }}$ was poorly correlated with the ETP and peak ( $r_{s}:-0.205$ to -0.532$)$. Hence, the variation in TG is likely caused by the prothrombin conversion and not the decay. Here again the individuals using oral contraceptives can be distinguished.

\section{DISCUSSION}

TG was shown to be affected by practically all anticoagulants ${ }^{39}$ and to reflect the overall coagulation better than clotting time-based assays. ${ }^{28}$ To our knowledge, this is the first study to assess the intra-individual variation of the effect of DOACs on global coagulation measured with TG over a longer period of time. Additionally, we confirmed the previously reported high inter-individual variation upon addition of a fixed concentration of DOACs to TG. ${ }^{29}$ From the presented data, we can conclude that the variation between individuals of the rivaroxaban and apixaban effect on TG is high but also, that this effect can vary within one subject in time, be it to a lesser extent.

Before discussing the variation, the effect of reversible FXa inhibitors on the pattern of TG curves should be reviewed. It is often observed that TG is prolonged in time and sometimes even a 'camelback' pattern is seen, as if two waves of TG follow each other. We surmise that the second wave is due to a lack of feedback inhibition of TF/FVIla by tissue factor pathway inhibitor (TFPI)/FXa. This makes that the peak is much more inhibited 
than the ETP and the correlation between peak and ETP deviates significantly from that in normal plasmas. ${ }^{29}$ These aspects are important to keep in mind when evaluating the variation in these parameters. The inter-individual variation in peak and ETP in the absence of DOACs was around 15\%, which is comparable to previous reports. ${ }^{29,40}$ Upon addition of rivaroxaban and apixaban this variation increased and was in a similar range as the CV'S we previously noted for otamixaban (ETP: $20.5 \%$, peak: $27.4 \%) .{ }^{29}$ The effect of the DOACs (\% inhibition) varied up to $44.5 \%$ for the ETP and $13.1 \%$ for the peak. These findings also confirmed the ones we reported earlier for otamixaban. ${ }^{29}$ Due to the particular shape of the curve and thus the larger effect on the peak than on the ETP, there is a larger variation in the remaining peak as compared to the ETP. Consequently, the average effect on the peak was $79 \%$ for rivaroxaban and $64 \%$ for apixaban, at $T 1$, and respectively $20 \%$ and $16 \%$ for the ETP. The concentration-effect relation for the anticoagulant effect on TG reaches a plateau phase faster for the peak than for the ETP. Looking at the response parameters (i.e. \% inhibition), the CV is therefore higher for the ETP as compared to the peak. ${ }^{29}$ The clinical impact of the change in shape of the TG curve by direct FXa inhibitors as rivaroxaban and apixaban is unclear and requires further evaluation in clinical studies. One of the strengths of this study was that all the measurements were performed in the same setting and in particular, that with each measurement of the inter-individual variation a NPP control was included. Therefore, we could correct the calculated CV's for the experimental variation of our assay.

High inter-individual variability in DOAC levels among patients must be attributed to pharmacokinetic effects (absorption, metabolism or drug elimination). ${ }^{18-20,29}$ Such variation is excluded from the present in vitro study that focusses on the 'pharmacodynamic' variation, i.e. the response of the donor to a fixed concentration of DOACs. The findings presented here could possibly offer an explanation for cases of treatment failure or bleeding when in the on-therapy plasma range. ${ }^{23,24}$ Kreutz et al. studied pharmacodynamic as well as -kinetic effects of rivaroxaban and apixaban in healthy volunteers over the course of seven days. ${ }^{41}$ They also presented a large standard deviation for both ETP and peak values between individuals, which is confirmed by our data on inter-individual variation. Although they followed donors over time, no results were reported on the intra-individual variation.

In this study, the intra-individual variation of the TG parameters in the absence of DOACs was comparable to those found in previous studies that described CV's of $4.5 \%$ to $12.5 \%$ 
for the endogenous thrombin potential (ETP), peak and lag time (at $5 \mathrm{pM}$ TF and $4 \mu \mathrm{M}$ $\mathrm{PL}){ }^{35,42}$ The variation increased in the presence of DOACs, for some individuals more than others. The majority of the twelve healthy donors showed a stable effect of both rivaroxaban and apixaban over the time course of one year. Interestingly, donors showing a large variation in response to rivaroxaban could differ from those showing a large variation in response to apixaban. This indicates that the variation found over time is not solely due to the characteristics of a donor; which could lead to the hypothesis that, based on the effects in plasma, one DOAC could be more suitable for an individual than another.

We also calculated prothrombin conversion and thrombin decay parameters (thrombin dynamics). ${ }^{38}$ The pattern of the variability of ETP and peak over time was similar to the pattern of $\mathrm{PC}_{\max }$ and $\mathrm{PC}_{\text {tot }}$ (i.e. the maximum prothrombin conversion rate and total prothrombin converted). The decay of thrombin remained relatively stable over time. This was confirmed by good correlations between the ETP and peak of TG and prothrombin conversion. The prothrombinase complex consists of factor $\mathrm{Xa}$, factor $\mathrm{Va}$ and prothrombin bound to a phospholipid surface and is thus affected by direct factor Xa inhibitors. The activity of the prothrombinase complex is reflected in the prothrombin conversion parameters. The thrombin decay is mainly determined by antithrombin and $\mathrm{a}_{2}$ macroglobulin, which is not affected by direct factor Xa inhibitors. ${ }^{38}$ These agents do affect feedback of factor Xa on tissue factor pathway inhibitor (TFPI), but this is not displayed in the thrombin decay constant.

Another remarkable observation was that two donors had a clearly higher TG (ETP and peak), higher prothrombin conversion and a lower response to rivaroxaban and apixaban. These individuals were the only donors using hormonal contraceptives. It is known that the use of hormonal contraceptives is correlated with a higher risk of thrombosis ${ }^{43}$ and acquired resistance to activated protein C (APC). ${ }^{44}$ Our group has previously shown that TG in these individuals is increased even in the absence of thrombomodulin ${ }^{45,46}$, which confirms our results shown here. Moreover, this trend was also seen in the prothrombin conversion parameters, suggesting that these donors have a more active prothrombinase complex and consequently a faster prothrombin conversion. A benefit of studying the pharmacodynamic effects is that multiple varying factors caused by pharmacokinetics were circumvented. The effects seen were large enough, however, to warrant further clinical studies. 
In summary, after in vitro addition of a fixed dose of rivaroxaban and apixaban to the plasma of healthy individuals the effect on TG proved to be highly variable between different individuals. The variation within individuals of effect on TG remained fairly stable over time for most of the donors, with some clear exceptions. The question whether a high variability in response to DOACs is associated with a higher risk of thrombosis or bleeding remains to be established in further research.

\section{ADDENDUM}

S. Zwaveling performed experiments, analyzed data and wrote the manuscript; J. Konings performed experiments; H. Kelchtermans designed research; H.C. Hemker analyzed data and reviewed the manuscript; S. Bloemen designed research, performed experiments, analyzed data and wrote the manuscript. All authors approved the final version of the manuscript.

\section{DISCLOSURE OF CONFLICTS OF INTEREST}

S. Zwaveling, J. Konings, H. Kelchtermans and B. de Laat are employed by Synapse Research Institute, that is part of Diagnostica Stago. S. Bloemen was employed by Synapse Research Institute in the past 36 months and reports personal fees from UFL/SWOL. H.C. Hemker is consultant for Synapse Research Institute and Diagnostica Stago. 


\section{REFERENCES}

1. Granger $\mathrm{CB}$, Alexander JH, McMurray JJ, et al. Apixaban versus warfarin in patients with atrial fibrillation. The New England journal of medicine. 2011;365(11):981-992.

2. Connolly SJ, Ezekowitz MD, Yusuf S, et al. Dabigatran versus warfarin in patients with atrial fibrillation. The New England journal of medicine. 2009;361(12):1139-1151.

3. Patel MR, Mahaffey KW, Garg J, et al. Rivaroxaban versus warfarin in nonvalvular atrial fibrillation. The New England journal of medicine. 2011;365(10):883-891.

4. Giugliano RP, Ruff CT, Braunwald E, et al. Edoxaban versus warfarin in patients with atrial fibrillation. The New England journal of medicine. 2013;369(22):2093-2104.

5. Lassen MR, Ageno W, Borris LC, et al. Rivaroxaban versus enoxaparin for thromboprophylaxis after total knee arthroplasty. The New England journal of medicine. 2008;358(26):2776-2786.

6. Schulman S, Kearon C. Definition of major bleeding in clinical investigations of antihemostatic medicinal products in non-surgical patients. Journal of thrombosis and haemostasis : JTH. 2005;3(4):692-694.

7. Perzborn E, Kubitza D, Misselwitz F. Rivaroxaban. A novel, oral, direct factor Xa inhibitor in clinical development for the prevention and treatment of thromboembolic disorders. Hamostaseologie. 2007;27(4):282-289.

8. Stangier J. Clinical pharmacokinetics and pharmacodynamics of the oral direct thrombin inhibitor dabigatran etexilate. Clin Pharmacokinet. 2008;47(5):285-295.

9. Raghavan N, Frost CE, Yu Z, et al. Apixaban Metabolism and Pharmacokinetics after Oral Administration to Humans. Drug Metabolism and Disposition. 2009;37(1):74-81.

10. Baglin T, Hillarp A, Tripodi A, Elalamy I, Buller H, Ageno W. Measuring Oral Direct Inhibitors (ODIs) of thrombin and factor Xa: A recommendation from the Subcommittee on Control of Anticoagulation of the Scientific and Standardisation Committee of the International Society on Thrombosis and Haemostasis. Journal of thrombosis and haemostasis : JTH. 2013.

11. Samuelson BT, Cuker A. Measurement and reversal of the direct oral anticoagulants. Blood Rev. 2017;31(1):77-84.

12. Cuker A, Siegal DM, Crowther MA, Garcia DA. Laboratory measurement of the anticoagulant activity of the non-vitamin K oral anticoagulants. Journal of the American College of Cardiology. 2014;64(11):1128-1139.

13. Hawes EM, Deal AM, Funk-Adcock D, et al. Performance of coagulation tests in patients on therapeutic doses of dabigatran: a cross-sectional pharmacodynamic study based on peak and trough plasma levels. Journal of thrombosis and haemostasis : JTH. 2013;11(8):1493-1502.

14. Francart SJ, Hawes EM, Deal AM, et al. Performance of coagulation tests in patients on therapeutic doses of rivaroxaban. A cross-sectional pharmacodynamic study based on peak and trough plasma levels. Thrombosis and haemostasis. 2014;111(6):1133-1140.

15. Samama MM, Guinet C, Le Flem L, Ninin E, Debue JM. Measurement of dabigatran and rivaroxaban in primary prevention of venous thromboembolism in 106 patients, who have undergone major orthopedic surgery: an observational study. Journal of thrombosis and thrombolysis. 2013;35(2):140-146.

16. Testa S, Tripodi A, Legnani C, et al. Plasma levels of direct oral anticoagulants in real life patients with atrial fibrillation: Results observed in four anticoagulation clinics. Thromb Res. 2016;137:178-183.

17. Chan NC, Coppens M, Hirsh J, et al. Real-world variability in dabigatran levels in patients with atrial fibrillation. Journal of thrombosis and haemostasis : JTH. 2015;13(3):353-359.

18. Gong IY, Kim RB. Importance of pharmacokinetic profile and variability as determinants of dose and response to dabigatran, rivaroxaban, and apixaban. Can J Cardiol. 2013;29(7 Suppl):S24-33.

19. Kubitza D, Roth A, Becka M, et al. Effect of hepatic impairment on the pharmacokinetics and pharmacodynamics of a single dose of rivaroxaban, an oral, direct Factor Xa inhibitor. Br J Clin Pharmacol. 2013;76(1):89-98. 
20. Reilly PA, Lehr T, Haertter S, et al. The effect of dabigatran plasma concentrations and patient characteristics on the frequency of ischemic stroke and major bleeding in atrial fibrillation patients: the RE-LY Trial (Randomized Evaluation of Long-Term Anticoagulation Therapy). Journal of the American College of Cardiology. 2014;63(4):321-328.

21. Aisenberg J, Chatterjee-Murphy P, Friedman Flack K, et al. Gastrointestinal Bleeding With Edoxaban Versus Warfarin: Results From the ENGAGE AF-TIMI 48 Trial (Effective Anticoagulation With Factor Xa Next Generation in Atrial Fibrillation-Thrombolysis In Myocardial Infarction). Circulation Cardiovascular quality and outcomes. 2018;11(5):e003998.

22. Testa S, Paoletti O, Legnani $\mathrm{C}$, et al. Low drug levels and thrombotic complications in high-risk atrial fibrillation patients treated with direct oral anticoagulants. Journal of thrombosis and haemostasis : JTH. 2018;16(5):842-848.

23. Ten Cate H, Olie RH, Ten Cate-Hoek AJ, Henskens YMC. Direct oral anticoagulants: When to consider laboratory testing? Int J Lab Hematol. 2018;40 Suppl 1:30-33.

24. Albaladejo P, Samama CM, Sie P, et al. Management of Severe Bleeding in Patients Treated with Direct Oral Anticoagulants: An Observational Registry Analysis. Anesthesiology. 2017;127(1):111-120.

25. Bloemen S, Zwaveling S, Ten Cate H, Ten Cate-Hoek A, de Laat B. Prediction of bleeding risk in patients taking vitamin K antagonists using thrombin generation testing. PloS one. 2017;12(5):e0176967.

26. Tripodi A, Legnani C, Chantarangkul V, Cosmi B, Palareti G, Mannucci PM. High thrombin generation measured in the presence of thrombomodulin is associated with an increased risk of recurrent venous thromboembolism. Journal of thrombosis and haemostasis : JTH. 2008;6(8):1327-1333.

27. Segers $\mathrm{O}$, van Oerle R, ten Cate H, Rosing J, Castoldi E. Thrombin generation as an intermediate phenotype for venous thrombosis. Thrombosis and haemostasis. 2010;103(1):114-122.

28. Hemker HC, Giesen P, AIDieri R, et al. The calibrated automated thrombogram (CAT): a universal routine test for hyper- and hypocoagulability. Pathophysiology of haemostasis and thrombosis. 2002;32(5-6):249-253.

29. Bloemen S, Hemker HC, Al Dieri R. Large inter-individual variation of the pharmacodynamic effect of anticoagulant drugs on thrombin generation. Haematologica. 2013;98(4):549-554.

30. Al Dieri R, de Laat B, Hemker HC. Thrombin generation: what have we learned? Blood Rev. 2012;26(5):197-203.

31. Favaloro EJ, Lippi G. Laboratory testing in the era of direct or non-vitamin $\mathrm{K}$ antagonist oral anticoagulants: a practical guide to measuring their activity and avoiding diagnostic errors. Semin Thromb Hemost. 2015;41(2):208-227.

32. Gatt A, van Veen JJ, Woolley AM, Kitchen S, Cooper P, Makris M. Thrombin generation assays are superior to traditional tests in assessing anticoagulation reversal in vitro. Thrombosis and haemostasis. 2008;100(2):350-355

33. Dinkelaar J, Patiwael S, Harenberg J, Leyte A, Brinkman HJ. Global coagulation tests: their applicability for measuring direct factor $\mathrm{Xa}$ - and thrombin inhibition and reversal of anticoagulation by prothrombin complex concentrate. Clinical chemistry and laboratory medicine : CCLM / FESCC. 2014;52(11):1615-1623.

34. van Ryn J, Grottke O, Spronk H. Measurement of dabigatran in standardly used clinical assays, whole blood viscoelastic coagulation, and thrombin generation assays. Clinics in laboratory medicine. 2014;34(3):479-501.

35. Hemker HC, Giesen P, Al Dieri R, et al. Calibrated automated thrombin generation measurement in clotting plasma. Pathophysiol Haemost Thromb. 2003;33(1):4-15

36. Hemker HC, Hemker PW, Al Dieri R. The technique of measuring thrombin generation with fluorescent substrates: 4. The H-transform, a mathematical procedure to obtain thrombin concentrations without external calibration. Thromb Haemost. 2009;101(1):171-177.

37. Bloemen S, Kelchtermans H, Hemker HC. Thrombin generation in low plasma volumes. Thromb J. 2018;16:10.

38. Kremers RM, Peters TC, Wagenvoord RJ, Hemker HC. The balance of pro- and anticoagulant processes underlying thrombin generation. Journal of thrombosis and haemostasis : JTH. 2015;13(3):437-447. 
39. Tripodi A, Padovan L, Veena C, Scalambrino E, Testa S, Peyvandi F. How the direct oral anticoagulant apixaban affects thrombin generation parameters. Thromb Res. 2015;135(6):1186-1190.

40. Hemker HC, Giesen P, Al Dieri R, et al. Calibrated automated thrombin generation measurement in clotting plasma. Pathophysiology of haemostasis and thrombosis. 2003;33(1):4-15.

41. Kreutz R, Persson PB, Kubitza D, et al. Dissociation between the pharmacokinetics and pharmacodynamics of once-daily rivaroxaban and twice-daily apixaban: a randomized crossover study. Journal of thrombosis and haemostasis : JTH. 2017;15(10):2017-2028.

42. Dargaud $Y$, Francillon $S$, Negrier $C$. Intraindividual thrombin generation measurement variability in healthy adults over a one year period. Thromb Res. 2009;124(2):237-238.

43. Fleischer K, van Vliet HA, Rosendaal FR, Rosing J, Tchaikovski S, Helmerhorst FM. Effects of the contraceptive patch, the vaginal ring and an oral contraceptive on APC resistance and SHBG: a cross-over study. Thrombosis research. 2009;123(3):429-435.

44. Tchaikovski SN, van Vliet HA, Thomassen MC, et al. Effect of oral contraceptives on thrombin generation measured via calibrated automated thrombography. Thrombosis and haemostasis. 2007;98(6):1350-1356.

45. Rotteveel RC, Roozendaal KJ, Eijsman L, Hemker HC. The influence of oral contraceptives on the time-integral of thrombin generation (thrombin potential). Thrombosis and haemostasis. 1993;70(6):959-962.

46. Bloemen S, Huskens D, J. K, et al. Interindividual Variability and Normal Ranges of Whole Blood and Plasma Thrombin Generation. The Journal of Applied Laboratory Medicine: an AACC Publication. 2017;2(2):150-164. 


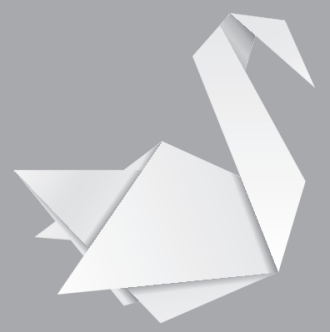




\section{CHAPTER}

The differential effects of dabigatran, rivaroxaban and apixaban on fibrinolysis

S. Zwaveling

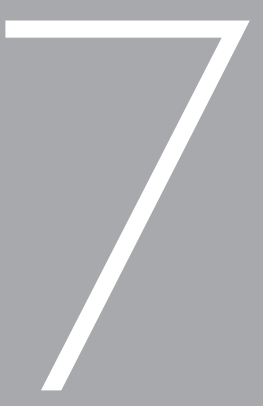

J. Konings

A. Miszta

H.C. Hemker

P.G. de Groot

S. Bloemen

In preparation 


\section{ABSTRACT}

\section{Background:}

The effects of direct oral anticoagulants (DOACs) on fibrinolysis are unclear. Thrombin improves the density of the fibrin network, activates factor XIII and thrombin-activatable fibrinolysis inhibitor (TAFI) via thrombomodulin (TM), forming a stronger clot. By reducing thrombin levels DOACs may facilitate clot lysis.

\section{Aim}

To study the effect of direct factor Xa (FXa) inhibitors and a direct thrombin inhibitor (DTI, dabigatran) on clot lysis.

\section{Methods}

Clot lysis time (CLT) was studied in 20 healthy donors using turbidimetry $(405 \mathrm{~nm})$ initiated with 5 pmol/L tissue factor (TF) and 100 IU tissue plasminogen activator (tPA). Plasma with or without dabigatran, rivaroxaban or apixaban at $I_{50}$ was measured in the presence and absence of TM and potato tuber carboxypeptidase inhibitor (PTCI). Simultaneously, thrombin generation (CAT) was assessed in the presence of TM.

\section{Results}

Dabigatran and direct FXa inhibitors shortened the clot lysis time (CLT) dose-dependently. TM prolonged the CLT significantly, in all conditions. This prolongation disappeared upon addition of PTCI, indicating a TAFI dependent mechanism. In 20 healthy donors, a dose-dependent acceleration of fibrinolysis was found by both DTI as well as by direct FXa inhibitors in the absence of TM. With TM present, this effect remained evident in conditions with dabigatran, but was limited for rivaroxaban and apixaban.

\section{Conclusion}

All DOACs accelerate fibrinolysis by diminishing thrombin activity. Rivaroxaban and apixaban decrease the CLT in the absence but not in the presence of TM, whereas dabigatran has a profibrinolytic effect in both conditions. We hypothesize that dabigatran can inhibit free thrombin as well as TM-bound thrombin, reducing TAFla and thereby promoting fibrinolysis. 


\section{INTRODUCTION}

Direct oral anticoagulants (DOACs) have received much attention since their introduction in the clinic. Large clinical trials have established both the efficacy and safety of DOACs for several thromboembolic diseases..$^{1-3}$ Consequently, they have become a cornerstone of oral anticoagulant therapy. Currently, two types of DOACs are approved worldwide: direct thrombin inhibitors (DTI) (e.g. dabigatran) and direct factor Xa (FXa) inhibitors (e.g. rivaroxban, apixaban and edoxaban). DOACs are frequently prescribed, not only to prevent stroke or systemic thromboembolism in patients with non-valvular atrial fibrillation (AF), but also to treat patients with venous thromboembolism (VTE). Although their mechanism of action focuses on different proteins in coagulation, both classes of DOACs eventually diminish thrombin activity. Dabigatran inhibits thrombin directly, whereas direct FXa inhibitors inhibit thrombin formation, by suppressing the conversion of prothrombin to thrombin.

Most attention is focused on the effects of DOACs on coagulation, but little attention has been paid to the effects of DOACs on fibrinolysis. Besides a key role in clot formation, thrombin is an important regulator of fibrinolysis. Thrombin is able to protect a fibrin clot against fibrinolysis in multiple ways. Firstly, a higher concentration of thrombin is related to thinner fibrin fibres that are tightly packed together in a denser fibrin network. ${ }^{4}$ Secondly, thrombin can activate factor XIII, which is responsible for crosslinking fibrin fibres and for the covalent binding of a2-antiplasmin to fibrin. ${ }^{5}$ Finally, thrombin can activate thrombin-activatable fibrinolysis inhibitor (TAFI); this is most efficient when thrombin is in complex with thrombomodulin (TM). Activated TAFI (TAFla) protects the clot against fibrinolysis by removing the $\mathrm{C}$-terminal lysines from partly degraded fibrin. These lysines are binding sites for tissue-type plasminogen activator (tPA) and plasminogen. TAFla thus impairs tPA mediated plasminogen conversion. 6,7 By reducing thrombin levels DOACs may indirectly facilitate clot lysis. The capacity to make fibrin clots more susceptible to fibrinolysis may represent an important additional mechanism that could contribute to the antithrombotic properties of DOACs. On the other hand, drugs that not only impair coagulation but also facilitate fibrinolysis may increase the risk of bleeding. This would support the findings of the LETS and MEGA studies, which both showed a relation between a decreased overall fibrinolytic potential and an increased risk of venous thrombosis. ${ }^{8-10}$ 
A few studies have investigated the relationship between DOAC and fibrinolysis, but reported conflicting results. Lisman et al. found that direct FXa inhibitors shorten the clot lysis time (CLT), in contrast to a bivalent DTI. ${ }^{11}$ However, dabigatran was not among the studied drugs. Another group showed enhanced fibrinolysis in the presence of dabigatran, but did not examine direct FXa inhibitors. ${ }^{12}$ The investigation of both types of DOAC, performed in more recent studies, resulted in contradicting findings as well: a study by Semeraro et al. established a reduction in CLT in the presence of dabigatran, whereas no profibrinolytic effect of direct FXa inhibitors was detected..$^{13}$ Königsbrügge et al. found a profibrinolytic effect of all DOACs on the CLT, but solely in the presence of $\mathrm{TM} .{ }^{14}$ From these studies it seems plausible that the differential effects of DOACs on fibrinolysis could be attributed to the activity of thrombin. Therefore, a possible explanation could be found by measuring thrombin generation (TG).

Several studies have tried to measure TG but encountered difficulties with measuring samples containing dabigatran, and no conclusive answers were found. In this study we assessed the effect of most currently used DOACs (dabigatran, rivaroxaban and apixaban) on clot formation as well as degradation by measuring turbidimetry and TG in parallel, under similar conditions. Due to the in vitro design of our study, we were able to use plasma not containing dabigatran in the calibrator wells of TG. The interference of dabigatran with the thrombin calibrator could thus be circumvented, resulting in more reliable effects. Moreover, low concentrations of TF and TM were used, to more closely resemble physiological conditions. To unravel the mechanism of action behind the effect of DOACs on CLT we studied the potential role of TM and TAFI.

\section{MATERIALS AND METHODS}

\section{Reagents}

Recombinant tissue factor (TF), Innovin, was supplied by Dade-Behring (Marburg, Germany). Synthetic phospholipids (PL) were purchased from Avanti Polar Lipids Inc. (Alabaster, AL, USA) and used in the form of vesicles consisting of phosphatidylserine, phosphatidylethanolamine and phosphatidylcholine (1:1:3, mol:mol:mol). As recombinant tissue plasminogen activator (tPA), Alteplase (Actilyse) was used, from Boehringer Ingelheim (Alkmaar, the Netherlands). Potato tuber carboxypeptidase inhibitor (PTCI) was obtained from Sigma (Milan, Italy). Recombinant thrombomodulin (TM) was purchased from U-Protein Express BV (Utrecht, the 
Netherlands). Activated protein C (APC) was a generous gift from dr. Regnault (Nancy université, France). TAFI deficient plasma was obtained from Stago BNL (Leiden, the Netherlands). The fluorogenic substrate Z-Gly-Gly-Arg-aminomethylcoumarin (ZGGR-AMC) was acquired from Bachem (Basel, Switzerland). The calibrator ( $\mathrm{a}_{2} \mathrm{M}-\mathrm{Ila}$ ) used during CAT was prepared according to standard method established by Hemker et al. ${ }^{15}$ The majority of the reagents were dissolved in HEPES buffers containing $5 \mathrm{mg} / \mathrm{ml}$ or $60 \mathrm{mg} / \mathrm{ml}$ bovine serum albumin (BSA5 and BSA60) and were prepared as reported in the standard method, mentioned above. ${ }^{15}$ Rivaroxaban (Xarelto ${ }^{\circledR}$ ) was from Bayer (Berlin, Germany); stock solutions of $10 \mathrm{mg} / \mathrm{ml}$ were prepared in dimethylsulfioxide (DMSO). Dabigatran (Pradaxa ${ }^{\circledR}$ ) was diluted in pure $\mathrm{HCl}$ and Apixaban (Eliquis ${ }^{\circledR}$ ) in DMSO. Both were purchased from Alsachim (Illkirch-Graffenstaden, France). All DOACs were further diluted in BSA5 buffer.

\section{Blood collection and plasma preparation}

Venous blood samples were collected from twenty healthy individuals ( 9 males, 11 females), after they had given their informed consent. Blood was drawn by venipuncture of the antecubital vein using a 21-gauge needle (Terumo) and collected into 0.109 M sodium citrate (9:1 v/v) tubes (Venosafe, Terumo, Heverlee, Belgium). Platelet poor plasma (PPP) was prepared directly after blood was drawn by double centrifugation at $2830 \mathrm{~g}$ for $10 \mathrm{~min}$ at room temperature. Plasma samples were aliquoted and frozen immediately at $-80^{\circ} \mathrm{C}$. Normal pooled plasma (NPP) was prepared from blood collected from 116 healthy volunteers. Plasma was pooled after an initial centrifugation step $(2,500 \mathrm{~g}, 5 \mathrm{~min})$, followed by ultracentrifugation at $100,000 \mathrm{~g}$ for $10 \mathrm{~min}$. Aliquots of $500 \mu \mathrm{l}$ were snap frozen in liquid nitrogen and stored at $-80^{\circ} \mathrm{C}$ until analysis.

\section{Measuring clot lysis time}

Fibrinolysis was studied in PPP, using a modified version of the turbidimetric assay as described by the group of Colucci (and Lisman). ${ }^{11,16}$ The changes in optical density of the samples were measured in duplicate at a wavelength of $405 \mathrm{~nm}$ at $37^{\circ} \mathrm{C}$ in a SpectraMax M2e kinetic microplate reader (Molecular Devices, USA). Measurements were carried out for at least 2 hours with intervals of 20 seconds. To initiate clot formation, $10 \mu \mathrm{l} \mathrm{TF/PL} \mathrm{reagent}$ were added to the wells ( $5 \mathrm{pmol} / \mathrm{L}$ and $4 \mu \mathrm{mol} / \mathrm{L}$ final concentration, respectively), together with either $10 \mu \mathrm{l}$ BSA5 buffer, $5 \mu \mathrm{l} \mathrm{TM} \mathrm{(1} \mathrm{nmol/L} \mathrm{final} \mathrm{concentration)} \mathrm{and/or} 5 \mu \mathrm{lPTCl}(10 \mu \mathrm{g} / \mathrm{ml}$ final concentration). Plasma samples were thawed at $37^{\circ} \mathrm{C}$ for at least $5 \mathrm{~min}$. Next, samples from single donors were spiked with a fixed concentration of DOACs and then added (80 
$\mu \mathrm{l})$ to the well. After $10 \mathrm{~min}$ of incubation at $37^{\circ} \mathrm{C}$, the reaction was started by the rapid, but careful, addition of $20 \mu \mathrm{l}$ preheated solution of $\mathrm{CaCl}_{2}$ (final concentration: $16.7 \mathrm{mM}$ ) and tPA (final concentration: $100 \mathrm{IU}(972.2 \mathrm{pmol} / \mathrm{L})$ ). CLT was defined as the time from half maximal fibrin formation to half maximal degradation. When assessing samples from healthy donors, all samples from one subject were measured simultaneously in one run to reduce variation.

The amount of TF, TM and TPA were selected based on titration curves with concentrations ranging from $0-5 \mathrm{pmol} / \mathrm{L}, 0-40 \mathrm{nmol} / \mathrm{L}$ and $0-125 \mathrm{U}$ ) respectively. The fixed DOAC concentrations were derived as follows: dose-response curves of DOAC concentrations (from 0-1000 nmol/L) and clot lysis time were established in NPP. From these curves the $\mathrm{IC}_{50}$ for each DOAC was determined in the presence and absence of $\mathrm{TM}$. The $\mathrm{IC}_{50}$ concentrations were estimated to be $100 \mathrm{nmol} / \mathrm{L}$ for both rivaroxaban and apixaban and $125 \mathrm{nmol} / \mathrm{L}$ for dabigatran in the absence of TM, and $200 \mathrm{nmol} / \mathrm{L}$ for rivaroxaban as well as apixaban and $500 \mathrm{nmol} / \mathrm{L}$ for dabigatran in the presence of TM. Two hundred nmol/L of dabigatran were included as well to compare one concentration for all DOACs. Additional measurements were executed in the presence or absence of $10 \mu \mathrm{APC}(1 \mathrm{nmol} / \mathrm{L}$ final concentration) to confirm the dependency of TAFI. Also, to analyse the effects of DOACs on clot formation and lysis, both TG and turbidimetry were performed in parallel.

\section{Calibrated automated thrombinography (CAT)}

TG was performed in duplicate in PPP using CAT as described previously by Hemker et al. ${ }^{15}$ In summary, calibrator wells both consisted of $20 \mu$ calibrator and $80 \mu$ l of plasma without DOAC. TG wells consisted of $10 \mu \mathrm{lTF} / \mathrm{PL}$ reagent (final concentrations of $5 \mathrm{pmol} / \mathrm{L}$ TF and 4 $\mu \mathrm{mol} / \mathrm{L} \mathrm{PL})$, and $10 \mu \mathrm{lTM}(1 \mathrm{nmol} / \mathrm{L}$ final concentration). Next, $80 \mu \mathrm{l}$ plasma, in the presence or absence of DOACs, were added to the TG wells. After 10 min of incubation at $37^{\circ} \mathrm{C}, 20 \mu \mathrm{l}$ of $\mathrm{CaCl}_{2}$ solution containing the fluorescent substrate were dispensed into the wells. Data were analysed with specialized software from Thrombinoscope (Maastricht, The Netherlands). To control for experimental variation all samples of one donor were measured in one run.

\section{Statistical analysis}

Statistical analyses were performed with Graphpad Prism software (version 5.00). The distribution of the data was estimated using descriptive statistics. A normal distribution was confirmed using the Shapiro-Wilk test. Data are represented as mean with standard deviation (SD). Differences between means were analysed using an unpaired t-test, e.g. 
between different DOAC concentrations. A p-value $<0.05$ was considered significant. Variances between multiple groups were compared using the one-way ANOVA with Bonferroni as post hoc test.

\section{RESULTS}

\section{Effects of DOACs on fibrinolysis}

As outlined in the introduction, the main purpose of this work was to examine the in vitro effects of DOACs on fibrinolysis, using turbidimetry. Both direct Flla as well as direct FXa inhibitors shortened the CLT in a dose-dependent manner in NPP (Figure 1). The addition of $1 \mathrm{nmol} / \mathrm{L}$ TM prolonged the CLT considerately, both in the presence and in the absence of DOACs, as can be observed from figure $2 \mathrm{~A}$.

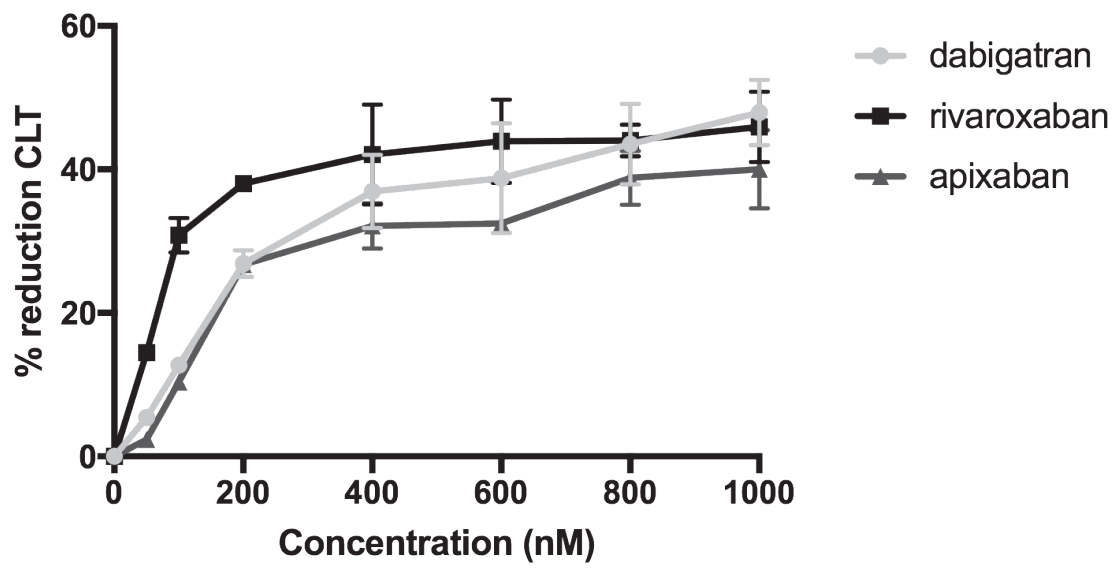

Figure 1. Dose response curves of the clot lysis time (CLT) in the presence of DOAC.

The inhibition of the CLT measured with turbidimetry in normal pooled plasma spiked with increasing concentrations of dabigatran, rivaroxaban and apixaban. Data are presented as mean \pm standard deviation (SD) $(n=2)$.

To show that the antifibrinolytic activity of TM is not attributed to the APC pathway, experiments were conducted in NPP in the presence of $1 \mathrm{nmol} / \mathrm{L}$ APC. The addition of APC did not prolong the CLT, but showed a cumulative anticoagulant effect in the presence of DOAC (data not shown). The prolongation of the CLT in the presence of TM was reversed by PTCI, a specific TAFI inhibitor (Figure 2B). Similar results were found in TAFI deficient plasma (data not shown). This indicates that the prolongation caused by this concentration of TM is TAFI-dependent. 

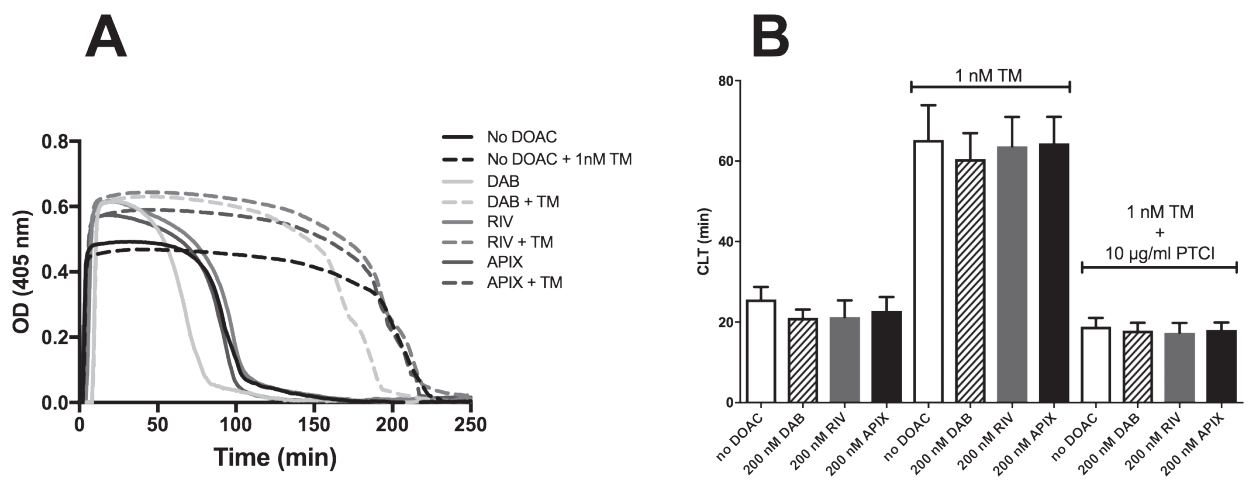

Figure 2. The effect of thrombomodulin (TM) and thrombin-activatable fibrinolysis inhibitor (TAFI) on the clot lysis time (CLT).

(A) CLT measured with turbidimetry in platelet poor plasma, with or without $200 \mathrm{nmol} / \mathrm{L}$ of dabigatran (DAB), rivaroxaban (RIV), apixaban (APIX), in the presence or absence of TM. (B) CLT in healthy donors $(n=20)$ in the presence or absence of $200 \mathrm{nmol} / \mathrm{L}$ dabigatran (DAB), rivaroxaban (RIV) and apixaban (APIX), with or without TM and potato tuber carboxypeptidase inhibitor (PTCI), a specific TAFI inhibitor. Data are represented as mean plus standard deviation (SD).

\section{Healthy controls}

The CLT was measured in twenty healthy donors in the presence and absence of TM. As illustrated in figure 3A, a dose-dependent acceleration of fibrinolysis can be observed for both the DTI as well as the direct FXa inhibitors in the absence of TM. When $125 \mathrm{nmol} / \mathrm{L}$ $\left(\mathrm{IC}_{50}\right)$ dabigatran was added, the CLT was reduced with $8.2 \%( \pm 4.4 \%)$. With the addition of $200 \mathrm{nmol} / \mathrm{L}$ dabigatran the lysis time shortened significantly by $17.3 \%$ ( $\pm 6.7 \%, p<0.0001)$. Dabigatran at a concentration of $500 \mathrm{nmol} / \mathrm{L}$ decreased the CLT even further with $28.5 \%$ $( \pm 8.5 \%, p<0.0001)$. For rivaroxaban we found a decrease in CLT of $8.9 \%( \pm 7.6 \%)$ and $17.0 \%$ $( \pm 11.6 \%)$ for $100 \mathrm{nmol} / \mathrm{L}\left(\mathrm{IC}_{50}\right)$ and $200 \mathrm{nmol} / \mathrm{L}$, respectively $(\mathrm{p}=0.015)$. For apixaban the effect on the CLT increased from 3.7\% ( \pm 4.2$)$ for $100 \mathrm{nmol} / \mathrm{L}\left(\mathrm{IC}_{50}\right)$ to $11.0 \%( \pm 6.3 \%)$ for $200 \mathrm{nmol} / \mathrm{L}$, $(p<0.001)$. In the presence of TM the dose-dependent decrease in CLT remained evident in plasma spiked with dabigatran (125 nmol/L: 0.04\% ( $\pm 3.6 \%) ; 200 \mathrm{nmol} / \mathrm{L}: 9.6 \%( \pm 5.9 \%)$; $500 \mathrm{nmol} / \mathrm{L}: 25.5 \%( \pm 7.4 \%),(p<0.0001))$, as presented in figure 4B. This effect, however, was muted in conditions with rivaroxaban and apixaban. Only a marginal acceleration of the mean CLT was found for both drugs: from $2.4 \%( \pm 4.3 \%)$ at $100 \mathrm{nmol} / \mathrm{L}$ to $4.9 \%( \pm 7.0 \%)$ at $200 \mathrm{nmol} / \mathrm{L}$ for rivaroxaban ( $\mathrm{p}=0.2)$ and from 2.4\% ( $\pm 2.4 \%)$ at $100 \mathrm{nM}$ to $3.9 \%( \pm 3.4 \%)$ at $200 \mathrm{nmol} / \mathrm{L}$ for apixaban ( $\mathrm{p=0.1)}$ (Figure 3B). 
A

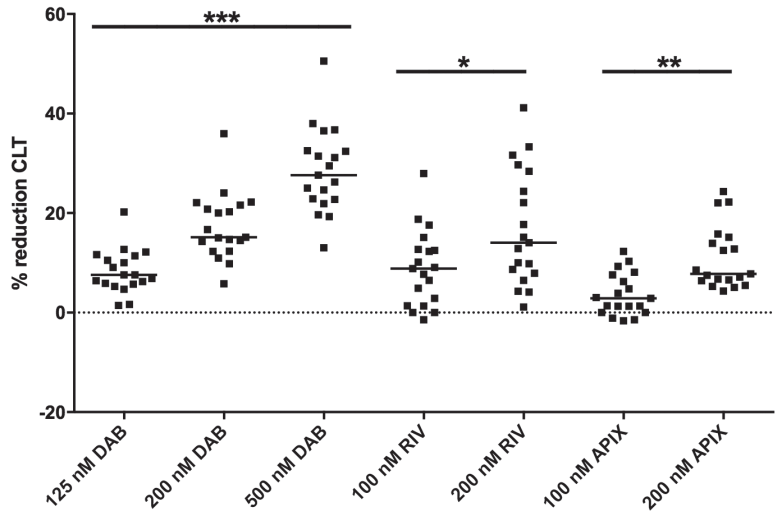

B

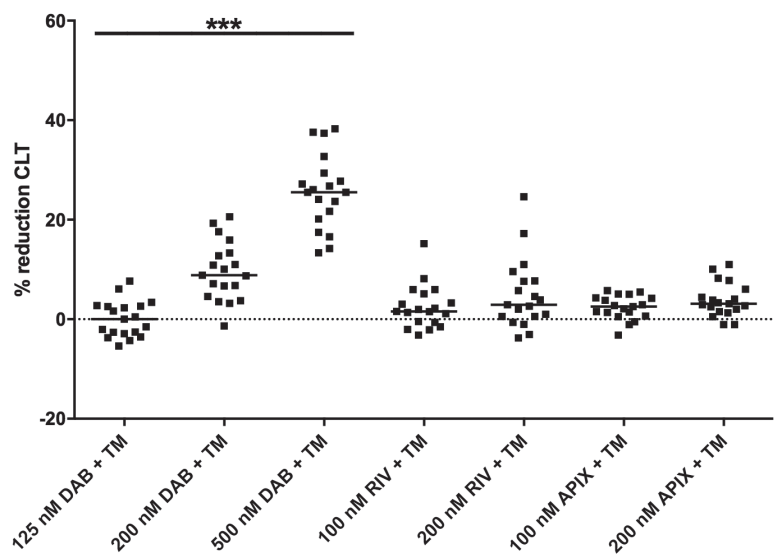

Figure 3. Reduction of the clot lysis time (CLT) by different concentrations of dabigatran, rivaroxaban and apixaban in healthy donors.

Inhibition of the CLT in plasma of healthy donors $(n=20)$ spiked with different concentrations of dabigatran (DAB), rivaroxaban (RIV) and apixaban (APIX). Turbidity measurements were performed in the absence (A) or presence (B) of thrombomodulin (TM)

\section{Effects of DOACs on TG}

To assess if the quantity of thrombin formed over time was equally supressed by DTI as by direct FXa inhibitors, TG was measured in the same healthy controls $(n=18)$. TG was performed in the presence of $1 \mathrm{nmol} / \mathrm{L} \mathrm{TM}$, to resemble the conditions in CLT. As displayed in figure 5 , the addition of $125 \mathrm{nmol} / \mathrm{L}$ dabigatran and $1 \mathrm{nmol} / \mathrm{L}$ TM increased the ETP in most of the cases (13 donors) with a mean increase of $4.1 \%( \pm 9 \%)$. When higher concentrations were tested $(200 \mathrm{nmol} / \mathrm{L}$ and $500 \mathrm{nmol} / \mathrm{L})$ the ETP was inhibited by $11.9 \%( \pm 10.4 \%)$ and 
$22.2 \%( \pm 11.4 \%)$, respectively, as would be expected. Adding $100 \mathrm{nmol} / \mathrm{L}$ rivaroxaban resulted in $18.9 \%( \pm 19,2 \%)$ decrease of ETP, and $200 \mathrm{nmol} / \mathrm{L}$ caused an inhibition of $31.7 \%( \pm 23.1 \%)$. The ETP was reduced by $13.6 \%( \pm 14.9 \%)$ at the addition of $100 \mathrm{nmol} / \mathrm{L}$ apixaban compared to $24.6 \%( \pm 16.2 \%)$ at $200 \mathrm{nmol} / \mathrm{L}$. All DOACs inhibited TG to a similar extent ( $p>0.3)$, when compared at the $\mathrm{IC}_{50}$ concentration of the CLT in the presence of TM $(500 \mathrm{nmol} / \mathrm{L}$ dabigatran; $200 \mathrm{nmol} / \mathrm{L}$ rivaroxaban and $200 \mathrm{nmol} / \mathrm{L}$ apixaban).

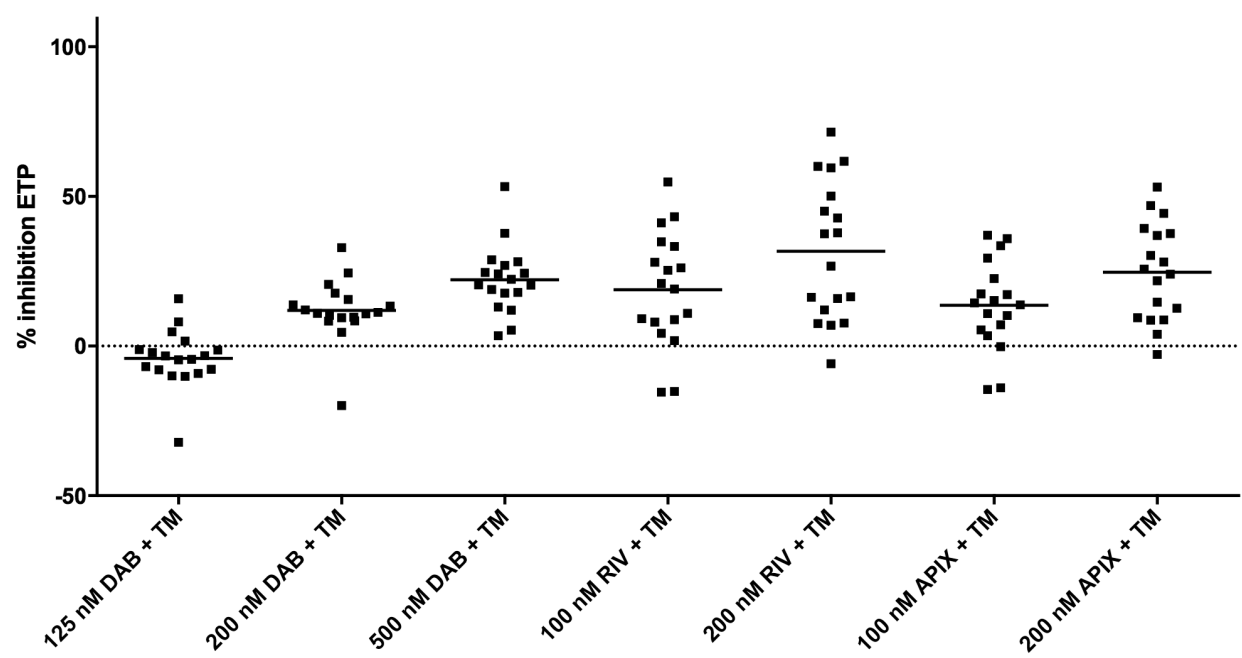

Figure 4. The effect of different DOAC concentrations on the endogenous thrombin potential (ETP) of thrombin generation (TG).

Inhibition of the ETP in plasma of healthy donors $(n=18)$, measured with TG in the presence of $1 \mathrm{nmol} / \mathrm{L}$ thrombomodulin (TM) spiked with dabigatran (DAB), rivaroxaban (RIV) and apixaban (APIX).

\section{DISCUSSION}

With this work we demonstrate that both, dabigatran (DTI), as well as the direct FXa inhibitors rivaroxaban and apixaban, accelerate fibrinolysis dose-dependently in the absence of TM. After the addition of TM, this dose-dependent decrease in the CLT remained evident in plasma spiked with dabigatran. Strikingly, this effect was muted in conditions with rivaroxaban and apixaban. By monitoring clot formation and degradation in a turbidimetric assay we demonstrated that all tested DOACs caused a dose-dependent reduction of the CLT, as anticipated.12,13,17,18 This effect can be attributed to the ability of DOACs to diminish thrombin activity resulting in an impeded clot formation. Additionally, a looser fibrin network allows for a better availability of tPA binding sites, thereby facilitating clot lysis. ${ }^{19,20}$ 
In line with previous research, we have demonstrated that the addition of a low concentration of TM prolongs the CLT considerably, both in the presence and the absence of DOACs. ${ }^{12-14}$ By measuring turbidity after addition of PTCI (a specific TAFI inhibitor), it could be shown that the profibrinolytic effect of TM under these conditions was caused by TAFla. Thrombin can form a complex with TM which, at low concentrations, stimulates the activation of TAFI into TAFla about a thousand times. ${ }^{21,22}$ TAFla protects the clot against tPA-mediated fibrinolysis. Prior studies have demonstrated markedly reduced amounts of TAFla in patients using dabigatran, in contrast to patients using direct FXa inhibitors. ${ }^{13}$ Consequently, it was suggested that these lower amounts of TAFla could be the result of lowered thrombin levels in patients using dabigatran compared to direct FXa inhibitors. ${ }^{13,14}$ Therefore, we assessed whether the quantity of thrombin formed over time was differently supressed by direct thrombin inhibitors as compared to direct FXa inhibitors by measuring TG under similar conditions as were used in the turbidity measurements. We established that both types of DOAC are able to inhibit TG substantially in the presence of TM. Moreover, there was no significant difference in thrombin formation between dabigatran and rivaroxaban or apixaban at equal molar concentrations. Due to the interference of dabigatran with the internal calibrator of CAT, prior studies were not able to accurately compare the amount of generated thrombin between the two types of DOAC. In our study, due to the in vitro design, we could use plasma that does not contain dabigatran in the calibrator wells, and could thereby circumvent most of the interference of dabigatran with TG. These findings confirm the results from previous studies showing the detection of similar levels of F1 + 2 and PAP complex in patients using different types of DOAC ${ }^{13}$. The present results imply that an alternative mechanism is responsible for the differential effects on CLT of DTI and direct FXa inhibitors.

Thrombin plays a central role in haemostasis, not only in coagulation, but also in fibrinolysis. Therefore, the regulation of its activity is of great importance. The role of thrombin (either stimulating or tempering coagulation) or its activity depends greatly on the ligand to which thrombin is bound. Thrombin has two positively charged domains that flank the active site of the molecule, exosite 1 and 2 , which regulate the binding of thrombin to substrates or cofactors. By binding a ligand, the exosites can either bring a substrate closer to the active site of thrombin, or facilitate the connection to an inhibitor. TM gains its cofactor abilities when bound in a 1:1 stoichiometric complex with thrombin via exosite 1. This connection to exosite 1 impairs access of other substrates to this particular exosite and reveals binding 
sites for protein C or TAFla. ${ }^{23}$ However, the occupation of exosite 1 leaves the active site of thrombin open. Dabigatran is a small molecule that binds selectively and reversibly to thrombin via the active site. It has been shown that dabigatran can not only inhibit soluble thrombin, but also thrombin bound to TM. ${ }^{24}$ Therefore, we postulate that the mechanism of action of DOACs on fibrinolysis is as follows: when dabigatran is present, continuous inhibition of thrombin-TM complex will reduce the activation of TAFI, which enhances fibrinolysis. Contrastingly, direct FXa inhibitors do not affect the thrombin-TM complex and therefore TAFI can still be activated, protecting the clot against lysis (Figure 6). Off course, the question remains in which way these in vitro observations will affect the clinical picture of patients receiving these classes of anticoagulants.

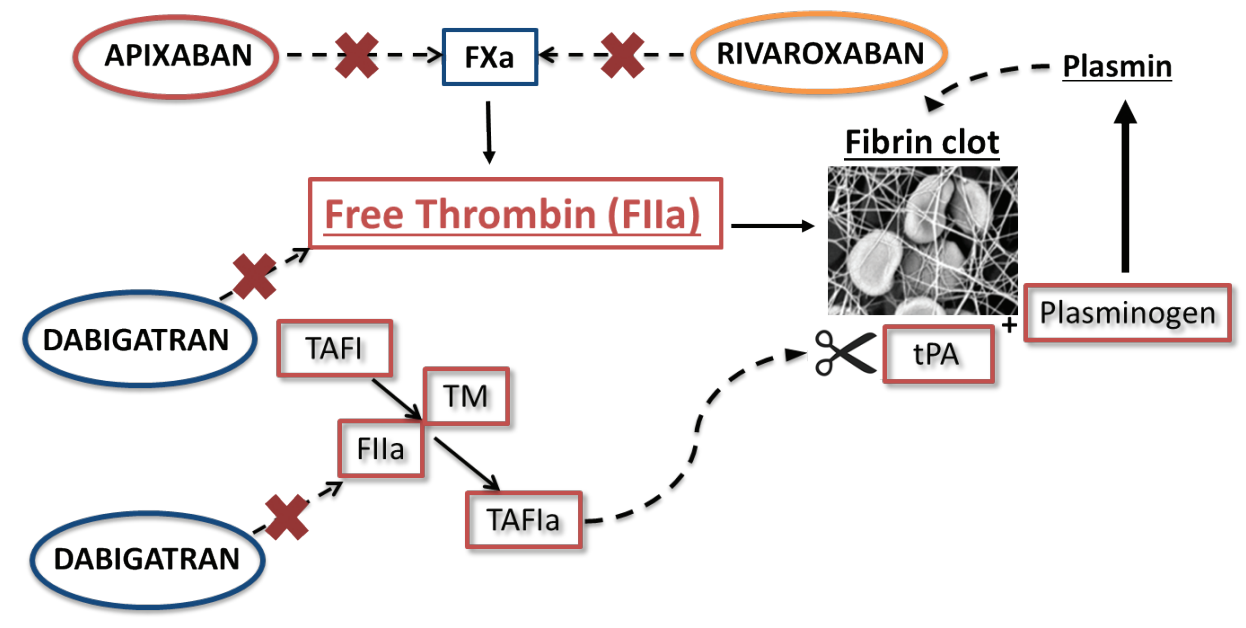

Figure 5. Proposed mechanism of the observed effects of direct thrombin and FXa inhibitors on fibrinolysis. Dabigatran inhibits not only free thrombin but also thrombin bound to thrombomodulin (TM), which leads to a reduced activation of thrombin activatable fibrinolysis inhibitor (TAFI), facilitating fibrinolysis. Direct FXa inhibitors do not affect the thrombin-TM complex. Thereby, TAFI activation is still feasible, protecting the clot from lysis.

This study has some limitations. When measuring TG in the presence of $125 \mathrm{nmol} / \mathrm{L}$ dabigatran we found an increased ETP in the majority of the cases, even after correction for dabigatran in the calibrator. This result, which would imply that dabigatran stimulates coagulation, is based on a known calculation artefact of TG software regarding dabigatran samples. ${ }^{25,26}$ Therefore, after the deduction of $\mathrm{a}_{2} \mathrm{M}$-thrombin from the total thrombin, an overestimation of the amount of free thrombin occurs. Nevertheless, the remaining artefact did not influence TG to the extent that the dose-dependent effect of dabigatran in TG was obscured. 
Although turbidity is a well-established method to measure CLT, is only reflects physiological conditions to a limited extent. In our experiments we used freshly thawed plasma and low amounts of TF and TM to approach an "in vivo" situation. It should be noted, however, that exogenous tPA was added at the start of the experiment, which could potentially interfere with the formation of the clot. To correct for inter-experimental variability, all conditions for one donor were assessed on the same plate, using the same plate layout over different runs.

In conclusion, we have shown that both types of DOACs can accelerate fibrinolysis by diminishing thrombin. Rivaroxaban and apixaban do decrease the CLT dose-dependently in the absence of TM, whereas this effect is limited when TM is present. Dabigatran has a clear profibrinolytic effect in both conditions. Dabigatran can inhibit free thrombin as well as thrombin bound to TM, reducing TAFla and thereby enabling more efficient clot lysis. Direct FXa inhibitors do not affect the thrombin-TM complex and therefore TAFI can still be activated, leading to better protection of the clot. Differential effects of DOACs on fibrinolysis, if confirmed in vivo, might influence clinical decision-making.

\section{ACKNOWLEDGEMENTS}

The authors like to thank Dr. H. Kelchtermans and Dr. B. de Laat for their contribution to the design of the study. Additionally, we would like to thank prof. H. ten Cate for his critical view in the discussion of the results. Furthermore, the authors are grateful to the volunteers that participated in this study.

\section{CONFLICT OF INTEREST STATEMENT}

All authors are linked to Synapse Research Institute, a research company part of Diagnostica Stago. S. Bloemen additionally reports personal fees from UFL/SWOL. H.C. Hemker and P.G. de Groot report personal fees from Diagnostica Stago. 


\section{REFERENCES}

1. Connolly SJ, Ezekowitz MD, Yusuf S, et al. Dabigatran versus warfarin in patients with atrial fibrillation. N Engl J Med. 2009;361(12):1139-1151.

2. Patel MR, Mahaffey KW, Garg J, et al. Rivaroxaban versus warfarin in nonvalvular atrial fibrillation. N Engl J Med. 2011;365(10):883-891.

3. Agnelli G, Buller HR, Cohen A, et al. Oral apixaban for the treatment of acute venous thromboembolism. N Engl J Med. 2013;369(9):799-808.

4. Wolberg AS. Thrombin generation and fibrin clot structure. Blood Rev. 2007;21(3):131-142.

5. Lorand L. Factor XIII: structure, activation, and interactions with fibrinogen and fibrin. Ann N Y Acad Sci. 2001;936:291-311.

6. Bajzar $\mathrm{L}$. Thrombin activatable fibrinolysis inhibitor and an antifibrinolytic pathway. Arterioscler Thromb Vasc Biol. 2000;20(12):2511-2518.

7. Mosnier LO, Bouma BN. Regulation of fibrinolysis by thrombin activatable fibrinolysis inhibitor, an unstable carboxypeptidase $B$ that unites the pathways of coagulation and fibrinolysis. Arterioscler Thromb Vasc Biol. 2006;26(11):2445-2453

8. Meltzer ME, Bol L, Rosendaal FR, Lisman T, Cannegieter SC. Hypofibrinolysis as a risk factor for recurrent venous thrombosis; results of the LETS follow-up study. J Thromb Haemost. 2010;8(3):605-607.

9. Lisman T, de Groot PG, Meijers JC, Rosendaal FR. Reduced plasma fibrinolytic potential is a risk factor for venous thrombosis. Blood. 2005;105(3):1102-1105.

10. Meltzer ME, Lisman T, Doggen CJ, de Groot PG, Rosendaal FR. Synergistic effects of hypofibrinolysis and genetic and acquired risk factors on the risk of a first venous thrombosis. PLoS Med. 2008;5(5):e97.

11. Lisman T, Adelmeijer J, Nieuwenhuis HK, de Groot PG. Enhancement of fibrinolytic potential in vitro by anticoagulant drugs targeting activated factor $X$, but not by those inhibiting thrombin or tissue factor. Blood Coagul Fibrinolysis. 2003;14(6):557-562.

12. Ammollo CT, Semeraro F, Incampo F, Semeraro N, Colucci M. Dabigatran enhances clot susceptibility to fibrinolysis by mechanisms dependent on and independent of thrombin-activatable fibrinolysis inhibitor. $J$ Thromb Haemost. 2010;8(4):790-798.

13. Semeraro F, Incampo F, Ammollo CT, et al. Dabigatran but not rivaroxaban or apixaban treatment decreases fibrinolytic resistance in patients with atrial fibrillation. Thromb Res. 2016;138:22-29.

14. Konigsbrugge $\mathrm{O}$, Weigel G, Quehenberger P, Pabinger I, Ay C. Plasma clot formation and clot lysis to compare effects of different anticoagulation treatments on hemostasis in patients with atrial fibrillation. Clin Exp Med. 2018.

15. Hemker HC, Giesen P, Al Dieri R, et al. Calibrated automated thrombin generation measurement in clotting plasma. Pathophysiol Haemost Thromb. 2003;33(1):4-15.

16. Colucci M, Binetti BM, Tripodi A, Chantarangkul V, Semeraro N. Hyperprothrombinemia associated with prothrombin G20210A mutation inhibits plasma fibrinolysis through a TAFI-mediated mechanism. Blood. 2004;103(6):2157-2161.

17. Semeraro F, Piro D, Rossiello MR, Ammollo T, Colucci M. Profibrinolytic activity of the direct thrombin inhibitor melagatran and unfractionated heparin in platelet-poor and platelet-rich clots. Thromb Haemost. 2007;98(6):1208-1214.

18. Nagashima H. Studies on the different modes of action of the anticoagulant protease inhibitors DX-9065a and Argatroban. II. Effects on fibrinolysis. J Biol Chem. 2002;277(52):50445-50449.

19. Gabriel DA, Muga K, Boothroyd EM. The effect of fibrin structure on fibrinolysis. J Biol Chem. 1992;267(34):24259-24263. 
20. Carr ME, Jr., Alving BM. Effect of fibrin structure on plasmin-mediated dissolution of plasma clots. Blood Coagul Fibrinolysis. 1995;6(6):567-573.

21. Mosnier LO, von dem Borne PA, Meijers JC, Bouma BN. Plasma TAFI levels influence the clot lysis time in healthy individuals in the presence of an intact intrinsic pathway of coagulation. Thromb Haemost. 1998;80(5):829-835.

22. Miah MF, Boffa MB. Functional analysis of mutant variants of thrombin-activatable fibrinolysis inhibitor resistant to activation by thrombin or plasmin. J Thromb Haemost. 2009;7(4):665-672.

23. Petrera NS, Stafford AR, Leslie BA, Kretz CA, Fredenburgh JC, Weitz JI. Long range communication between exosites 1 and 2 modulates thrombin function. J Biol Chem. 2009;284(38):25620-25629.

24. Cunanan J. Effect Of Dabigatran and Rivaroxiban On Thrombomodulin Mediated Activation Of Protein C and Thrombin Activated Fibrinolysis Inhibitor (TAFI). Potential Clinical Implications. Blood. 2013;122(21):3641.

25. Bloemen S, Zwaveling S, Douxfils J, Roest M, Kremers R, Mullier F. The anticoagulant effect of dabigatran is reflected in the lag time and time-to-peak, but not in the endogenous thrombin potential or peak, of thrombin generation. Thromb Res. 2018;171:160-166.

26. Wagenvoord RJ, Deinum J, Elg M, Hemker HC. The paradoxical stimulation by a reversible thrombin inhibitor of thrombin generation in plasma measured with thrombinography is caused by alpha-macroglobulin-thrombin. J Thromb Haemost. 2010;8(6):1281-1289. 


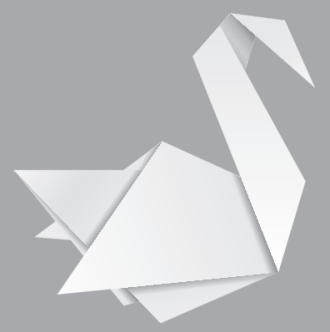




\section{CHAPTER | \\ Summary, general discussion and future prospects

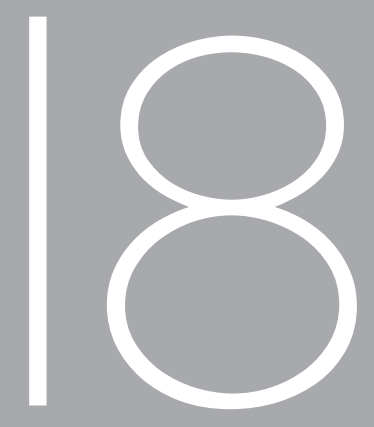




\section{SUMMARY AND GENERAL DISCUSSION}

\section{Introducing new drugs introduces new challenges}

Coagulation is a vital and highly complex system that maintains the dynamic balance between thrombosis and bleeding. Thrombosis is a common disease that is associated with a high rate of co-morbidity and mortality. In the Netherlands, about 1.5 million patients are being treated with anticoagulants for either arterial or venous thrombosis. The primary and most feared side effect of anticoagulant treatment is bleeding. The expected incidence of clinically relevant bleeding per year, for patients using oral anticoagulants, is $10-17 \%$. Additionally, anticoagulant drugs are frequently involved in complications or hospital admissions due to $\mathrm{ADE} .^{2,3}$ Most of these ADE's are caused by human errors and are thus potentially preventable. Therefore, correct management of anticoagulant treatment is of great importance. In the Netherlands, oral anticoagulant treatment of outpatients is managed by specialized, well-organized anticoagulation clinics. These centres have great expertise, not only in monitoring patients treated with VKA, using regular PT-tests to determine the right personal dosage for each patient, but also in educating patients and physicians.

The last decade thrombosis treatment has evolved. DOACs have driven VKAs from their dominant position and are now more frequently prescribed. ${ }^{4}$ These drugs are claimed to have stable pharmacokinetics and -dynamics and a short half-life. They are prescribed in a fixed dose based on indication and patient characteristics. Routine monitoring of these anticoagulants is considered unnecessary and is explicitly discouraged by guidelines. Thus, where other fields of medicine are developing towards a more personalized approach to treatment, thrombosis and haemostasis seems to be moving in the opposite direction.

The introduction of DOACs has introduced new challenges. Currently, there is no standardized follow-up of patients using DOACs within the Netherlands. Since monitoring of these drugs is discouraged, these patients are not referred to anticoagulation clinics. Follow-up is now mostly performed by GP's, who have limited experience in anticoagulant management due to the traditional, prominent role of anticoagulation clinics. The loss of regular visits to anticoagulation clinics, could lead to reduced patient education and coaching and perhaps further undermine therapy adherence. Even though regular monitoring of DOACs is not recommended, consensus is growing regarding the measurement of (the effect of) DOACs on certain occasions. ${ }^{5-8}$ The quick and easily available clotting time assays that are generally 
used to test coagulation (e.g. the PT or the aPTT) often lack the required sensitivity to assess the effect of DOACs. Their sensitivity is highly dependent on the reagent used in the laboratory. Hence, a normal aPTT with dabigatran or a normal PT with a direct FXa inhibitor does not exclude the presence of potentially relevant drug levels. Moreover, these clotting time tests have a more fundamental limitation. They solely measure the time from coagulation activation until the first traces of thrombin (time until clot is formed), disregarding the impact of extra feedback loops and the role of natural protein $\mathrm{C}$ and $\mathrm{S}$. Furthermore, they are not capable to assess hypercoagulability. This reveals a need for the development of more functional tests that are able to assess global coagulation. Ideally, such test could also detect the presence of DOACs and measure their effect on coagulation.

\section{Thrombin generation to investigate the risk of bleeding}

TG is a global coagulation assay that measures the total amount of thrombin that can be formed by an individual over time. ${ }^{9}$ In contrast to clotting time assays, TG represents a more complete view on the thrombotic tendency by measuring the full (functional) activity of thrombin. High amounts of thrombin are associated with an increased risk of (recurrent) thrombosis..$^{10-12}$ In contrast, patients who suffer from haemophilia, with an increased risk of bleeding, have an impaired TG compared to healthy controls. ${ }^{13,14}$ Additionally, a lower TG is associated with increased blood loss after cardiac surgery. ${ }^{15}$

Patients who are treated with VKAs have an increased risk of bleeding. Although these patients are monitored by regular INR measurements, bleeding can still occur. Patients using VKAs have a lower TG compared to patients without anticoagulants, although results on the correlation between the ETP and the INR are conflicting. 13,16,17 Previous studies have shown that TG measured in PPP is indicative of a risk of bleeding in different populations. ${ }^{15,18}$ TG can also be measured in PRP or in whole blood using CAT. The latter method includes the contribution of platelets, red and white blood cells, reflecting a more physiological environment. In the whole blood CAT, erythrocytes and platelets can function as a source of procoagulant phospholipids, thereby directly contributing to TG. In chapter 2 we investigated whether TG can identify a bleeding risk in 129 patients treated with VKAs. TG was measured in PPP, PRP and in whole blood in the presence and absence of TM. In addition, the risk of bleeding was calculated for each patient using the HAS-BLED score, which is the most common clinical decision score used to assess the risk of bleeding in patients with AF and has the highest predictive potential. ${ }^{19}$ We found that whole blood ETP and peak predicted bleeding 
as well as the HAS-BLED score with comparable areas under the ROC-curve. Implementing whole blood CAT in bleeding scores such as the HAS-BLED might theoretically improve their sensitivity, enabling further tailoring of therapy for individual patients using VKA. In contrast to previous studies we found a non-significant decrease in PPP ETP and peak in patients with bleeding compared to non-bleeding patients. The addition of TM, to make the TG assay more sensitive to the APC-system, did not change these results.

To further assess the association between TG and the risk of bleeding in patients using anticoagulants, we performed a systematic review of the literature (chapter $\mathbf{3}$ ). Although CAT is frequently used to assess the effects of anticoagulation in different research settings, remarkably few studies have investigated the direct relation between TG and clinical bleeding in stable, anticoagulated patients. Only seven studies could be included; two studies were of good methodological quality, others moderate. All papers studied VKAs only and most of them confirmed decreased TG values in patients compared with healthy controls. 16,18,20-24 The studies of highest quality found evidence supporting an association between low CAT-TG values and bleeding..$^{18,20}$ Other reviewed studies supported this reasoning, but did not provide enough data to validate this hypothesis. Unfortunately, no papers investigated the relation between TG and bleeding in patients using DOACs. In part, this may be due to technical limitations in measuring direct thrombin inhibitors (DTI) with TG, showing a paradoxical increase in peak and ETP. ${ }^{25-30}$ Otherwise, at the time of review, there were still no published data on measurements of direct Xa inhibitors with TG, in relation to bleeding complications. Interestingly, numerous papers have documented TG to assess the reversal of DOACs. ${ }^{31-39}$ These studies all assumed an indirect connection between TG results and the risk of bleeding (e.g. inadequate coagulation). Such studies also highlight the importance to look for associations between TG activity and bleeding outcomes in patients using DOACs. The use of fixed doses of DOACs, based solely on patient characteristics, causes a wide variability in their anticoagulant responses ${ }^{40,41}$ which makes testing with global coagulation assays like TG potentially interesting.

\section{The effect of direct thrombin inhibitors on CAT}

In assessing anticoagulants, TG measured with CAT is one of the most informative and sensitive tests. As described in chapters 2 and 3, the TG curve in response to VKAs shows a prolongation of the lag time and the time-to-peak, and a decrease in ETP and peak as a result of a diminished generation of thrombin. Previous studies have shown that UFH, 
LMWH, fondaparinux, VKA and also direct FXa inhibitors inhibit TG peak and ETP, although not always to the same extent. ${ }^{39,42}$

As mentioned before, dabigatran, a DTI, prolongs the lag time and time-to-peak during TG, but also shows an increased peak and ETP. This could bring about the misleading suggestion that the addition of thrombin inhibitor would increase the formation of thrombin in vivo. In chapter 4 we further investigated this paradoxical effect, also described by other groups $25,26,29,30,43$, and demonstrated that TG measurements in samples containing dabigatran are hampered by two mechanisms:

1. Most of the artificial increase in ETP and peak is caused by the inhibition of the calibrator activity by dabigatran. The calibrator used in CAT is the $\mathrm{a}_{2} \mathrm{M}$-thrombin complex. Although, thrombin is bound in this complex, the active site of the molecule is still available. Dabigatran is a small molecule and can therefore still attach to the active site, inhibiting thrombin when bound in the complex as is presented in Figure 2 and 3.44,45 Calibration in CAT is based on the simultaneous measurement of a known thrombin concentration. Subsequently, diminishing this concentration (by the DTI) leads to an overestimation of calculated thrombin activity in the TG well. To overcome this artefact, multiple groups have used donor plasmas in the TG well, and normal pooled plasma (NPP) (without dabigatran) in the calibrator wells. ${ }^{26,43,46}$ This method eliminates one of the benefits of CAT-TG: that samples are calibrated with their own plasma. It is known that the colour and the unique composition of plasma will affect the results of TG. ${ }^{47}$ We have neutralized the effect of dabigatran on the calibrator by the addition of the specific reversal agent idarucizumab, to the calibrator well. Studies have shown that administration of idarucizumab successfully restores coagulation in patients using dabigatran. ${ }^{48,49}$ When TG is measured with dabigatran in the presence and absence of idarucizumab in the calibrator well, the elevation in peak and ETP are only partly corrected, revealing a second, underlying artefact. 


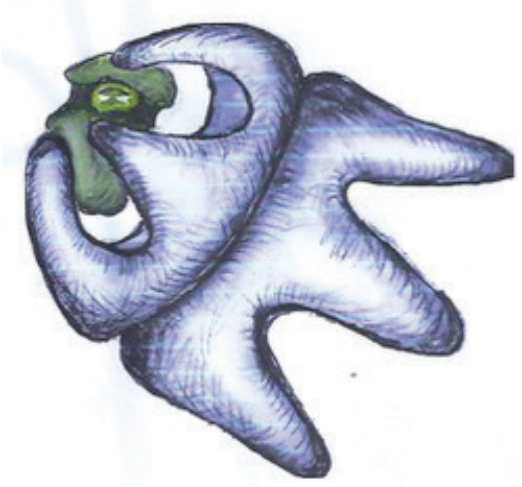

Figure 1. a2-macroglobulin molecule forming a complex with thrombin. The active site of thrombin is open. Figure made by Coen Hemker.

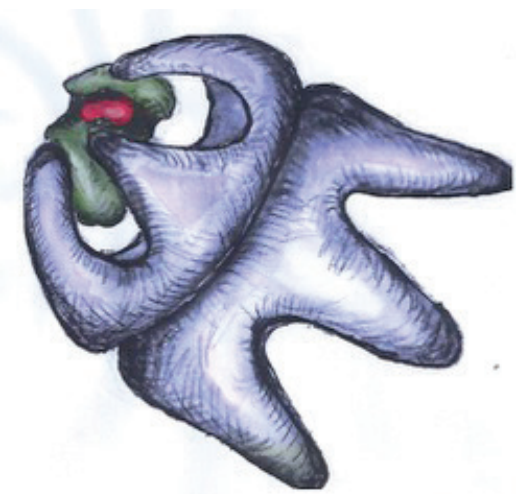

Figure 2. a2-macroglobulin molecule in complex with thrombin.

Dabigatran (in red) is bound to the active site of thrombin. Figure made by Coen Hemker.

2. From the newly generated thrombin in the TG well during CAT, $75-80 \%$ is permanently eliminated by AT and $20-25 \%$ binds to $\mathrm{a}_{2} \mathrm{M}$. $^{50,51}$ Thrombin in complex with $\mathrm{a}_{2} \mathrm{M}$ can still cleave the fluorescent substrate. Therefore, a standard correction algorithm is used which subtracts the fluorescence generated by $a_{2} M$-thrombin activity, from the total thrombin activity (i.e. free thrombin plus $\mathrm{a}_{2} \mathrm{M}$-thrombin). This calculation does not take into account that $\mathrm{a}_{2} \mathrm{M}$-thrombin is inhibited by dabigatran throughout the TG measurement. Consequently, less $\mathrm{a}_{2} \mathrm{M}$-thrombin is subtracted from total measured thrombin leading to an overestimation of TG, which is especially visible at low dabigatran concentrations. In this chapter we show that lag time and time-to-peak provide an accurate representation of the anticoagulant effect of dabigatran, in contrast to the functional parameters (peak, ETP and velocity index). When idarucizumab is added in both the calibrator and the TG well, these parameters can assess the "uninhibited TG", mimicking a situation of coagulation in a patient, in absence of dabigatran. In order to measure the effect of dabigatran on coagulation with TG, without overestimation, correcting the inhibition of the calibrator is an important first step, but will not solve the second artefact. Further research into possible solutions concerning the CAT algorithm is required to reliably assess the anticoagulant effect of dabigatran with CAT. 


\section{The effect of direct factor Xa inhibitors on thrombin generation}

The normal TG cure is characterized by a particular "bell shape" with a steep slope and a high peak. All anticoagulants tested so far affect this TG curve dose-dependently. 39,42,52,53 Most types of anticoagulants have a more profound effect on the peak than on the ETP. The most pronounced are the direct FXa inhibitors, which induce a more "protracted" TG curve, characterized by a long plateau phase. ${ }^{30,54-56}$ This results in a strongly decreased peak, but a relatively less inhibited ETP. In contrast to TG in the presence of DTI, technical or mathematical artefacts do not hamper TG in the presence of direct FXa inhibitors. The mechanism behind the protracted curve is not yet fully understood. It was hypothesized by Bloemen et al. that the first part of the TG curve is shaped by feedback of the Josso Ioop, i.e. the activation of FIX by TF/FVIIa. ${ }^{57}$ We postulate that the plateau phase could be the result of reduced activation of TFPI by FXa. The binding of FXa to TFPI controls the extrinsic pathway via TF. When the inhibition of TF is limited trough lower amounts of FXa, TG via the extrinsic pathway can continue for a longer period. Interestingly, in chapters 6 and 7, a different pattern of TG suppression is observed for different direct FXa inhibitors as well, despite similar mechanisms of action. Rivaroxaban suppresses the peak, but mostly the ETP, to a greater extent than apixaban. ${ }^{58} \mathrm{Kim}$ et al. have shown that both inhibitors had a similar affinity for $\mathrm{FXa}$ with comparable $\mathrm{K}_{\mathrm{i}}$ values at equilibrium. Kinetic measurements have revealed that rivaroxaban was a 4-fold more potent inhibitor of FXa. The same authors have studied TG to monitor the inhibition of prothrombinase and found that the association rates for the binding of rivaroxaban to either free FXa or incorporated FXa were 10- and 1.2- fold faster than those of apixaban, respectively. Interestingly, these findings suggest that rivaroxaban and apixaban differ in their capacity to inhibit free and bound FXa. We postulate that these inhibitors could also differ in their affınity for TFPI-FXa.

The different shape of the TG curve caused by different direct FXa inhibitors complicates interpretation of the test. Due to a lack of studies correlating TG in patients using direct FXa inhibitors with bleeding, the clinical impact of the TG curve remains to be further elucidated. When looking at the whole curve it is clear that the process of thrombin formation is impaired (Figure 2). However, looking at separate parameters could trigger conflicting assumptions: a prolonged lag time, time-to-peak and a suppressed peak would suggest that less thrombin is generated, whereas a barely changed ETP would suggest otherwise. Perhaps, combining multiple parameters would help to give more accurate information on clinical outcome. This would be an interesting and important topic for further research. 


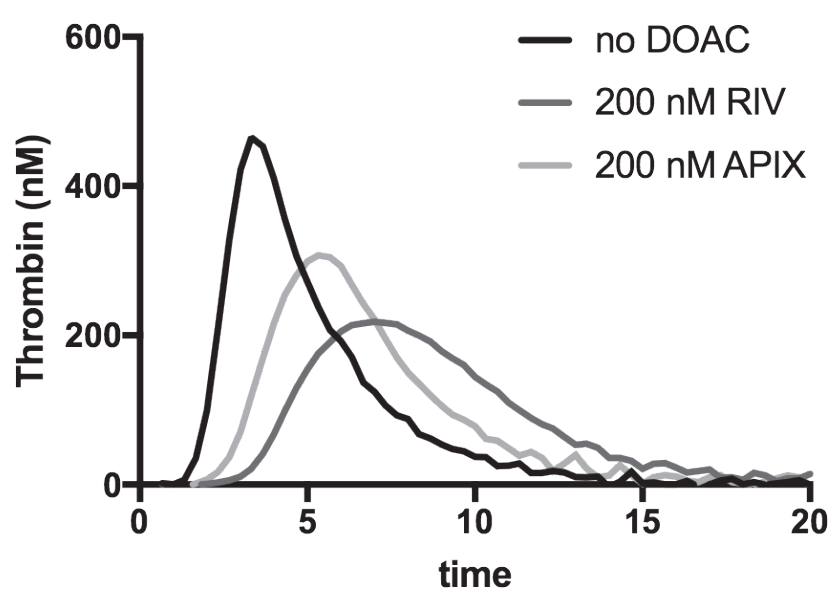

Figure 3. Thrombograph from one donor without direct oral anticoagulants (DOACs) and with $200 \mathrm{nM}$ rivaroxaban (RIV) or apixaban (APIX).

\section{A functional assay to measure DOACs}

There is increased consensus that measuring DOACs in certain clinical scenarios could optimize treatment and increase patient safety. ${ }^{5,7,8,59}$ Therefore, reliable assays are needed. Currently, all clotting time-based assays lack the required sensitivity and/or a good correlation between clotting time prolongation and anticoagulant intensity. ${ }^{8,60-65}$ Specialized assays (e.g. standardized calibrated chromogenic anti-FXa assays, the diluted thrombin time, or liquid chromatography tandem mass spectrometry) are able to give an accurate quantification of DOAC levels. ${ }^{54,65-68}$ These tests are not yet widely available and their availability and turn-around time are still hurdles in clinical practice, certainly in case of emergencies, like acute bleeding, surgery, or indications for thrombolysis, e.g. ischemic stroke. Furthermore, the interpretation of drug levels is complicated, because they are highly influenced by the moment of intake and the short half-life time of the drugs. Multiple studies found that drug levels differ widely between patients. ${ }^{40,41,69,70}$ Similarly to the situation with VKAs, bleeding or thrombotic events still occurred in spite of DOAC levels within the on-therapy range. ${ }^{71,72}$ Even though some studies have found an association between drug levels and clinical outcomes, more research is needed to define the strength of this risk association. ${ }^{73-75}$ Moreover, drug levels do not reflect the anticoagulant effect of DOACs. The impact on global coagulation should therefore be assessed with a functional assay. As bleeding or thrombotic complications may not be systematically associated to the drug levels, a combined measurement of levels and global haemostatic activity with TG might be more informative. 
In chapter 5 we developed a test to measure levels of rivaroxaban and concomitantly their effect on global coagulation. Xross-CAT is based on the capacity of rivaroxaban to dose-dependently inhibit free FXa as well as FXa in complex with $a_{2} M$, via the same mechanism as described in chapter 4. Using calibration curves and two different concentrations of $\mathrm{a}_{2} \mathrm{M}-\mathrm{FX}$, the Xross-CAT can detect rivaroxaban levels over a large range of concentrations (44 to $436 \mathrm{ng} / \mathrm{ml}$ ), relevant in clinical settings. The major advantage of this assay is that the detection of levels is performed in the CAT set-up, creating the possibility to determine not only drug levels, but also measure overall effect of those levels on coagulation activity with TG. Although not shown in this thesis, the principle of this test is also applicable to other direct FXa inhibitors as apixaban and edoxaban. The detection of levels is also possible for dabigatran provided that $\mathrm{a}_{2} \mathrm{M}$-Ila is used, but TG measurement has proven to be difficult. In chapter 5 we found a good correlation between the rivaroxaban levels measured with XrossCAT and with the Biophen DiXal, a chromogenic calibrated anti-FXa assay, currently used in the clinic. Plasma levels evaluated with Xross-CAT were slightly higher in general, which could be due to the different calibrators that are used in the two assays. The correlation with the PT and DRVV was moderate as expected, and comparable with other established correlations between quantitative- and clotting time-based assays. ${ }^{67}$ The Xross-CAT has an acceptable variability below $12 \%$ for drug levels of $44 \mathrm{ng} / \mathrm{ml}$ (i.e. $100 \mathrm{nM}$ ) and up. Lower levels of $22 \mathrm{ng} / \mathrm{ml}$ resulted in high CV's and were therefore excluded from measurements. This is a drawback, for example, when thrombolysis is considered and clinically relevant drug levels should be excluded. Another limitation is the current turn-around time of 50 min, which is primarily due to the centrifuging phase of blood to PPP. The Xross-CAT levels showed a hyperbolic relation with the functional TG parameters ETP and peak. This shows that low levels of rivaroxaban are sufficient to reduce the amplification phase of thrombin considerably. Similarly, at high concentrations the extent of the effect on the peak and ETP is relatively small. The non-linear relation between drug levels and ETP and peak, underlines the necessity to assess both aspects. Registries and cohort studies are needed to evaluate whether there is a net benefit for patients to measure the effect of DOACs on coagulation in addition to DOAC levels.

\section{The stability of DOAC effect between and within individuals over time}

As previously mentioned, the pharmacodynamic and -kinetic effects of DOACs are claimed to be stable. ${ }^{76-78}$ Therefore routine laboratory monitoring is discouraged. Dose adjustments are solely based on specific indications and patient characteristics. Nevertheless, measurement 
of DOACs on certain occasions might be helpful. 5,59 The latest guidelines suggest DOAC monitoring in specific situations, including major bleeding or thrombosis while on therapy, when considering thrombolysis, before urgent/elective surgery, when patients have decreased renal or liver function, are under- or overweight, or in case of long term drug exposure. ${ }^{8,79}$ However, these drug levels have proven to differ widely between as well as within patients. ${ }^{40,41,74,80-82}$ Moreover, drug levels are highly dependent on the type of DOAC, the dosage and, most importantly, the time since last intake. This large variation in levels observed in the large clinical trials with DOACs against warfarin has resulted in broad "on therapy" ranges. ${ }^{64,83-85}$ Real life studies have reported the highest variation between patients using the reduced dose of DOAC, which are often considered the most vulnerable group. ${ }^{86}$ Generally, median trough levels vary 6 - to 11 -fold between individuals. ${ }^{87}$ A within-patient variability expressed as the CV between $20 \%$ and $55 \%$ is reported depending on the time of testing (peak or trough) and the population. Instability of DOAC levels within patients indicates that single measurement of drug levels cannot accurately identify patients with consistently high or low levels and suggests the requirement of repeated measurements. The cause of this variation is sought in altered absorption, metabolism or drug-drug interactions but remains uncertain. Although the large variation in DOAC levels is known, it is not considered a reason for laboratory testing or individual dose adjustment. The main reason to refrain from laboratory test-based dose adjustment is the argument that in the large DOAC trials this was not needed to achieve efficacy and safety, globally comparable to dose adjusted VKAs. The question remains whether DOAC therapy could be further improved by laboratory guided DOAC or dose selection. Not for permanent monitoring but for optimizing drug and dose selection in the individual patient (as there is a choice in DOACs, there is a range of DOACs and doses to consider). A related issue is the type of test and whether the effects of highly variable DOAC concentrations are sufficiently measured in the available quantitative assays. As discussed before, although there are associations between levels and clinical outcomes, drug levels alone do not accurately represent the biochemical anticoagulant effects of DOACs. For this purpose, functional assays like TG may be additionally helpful. Studies on the stability of the anticoagulant effect of DOACs over time are scarce.

In chapter 6 we describe the variation of the effect of a fixed dose (in vitro) of rivaroxaban and apixaban on TG between individuals as well as within individuals over the period of one year. Our data confirm previously reported data on the high inter-individual variation of a direct FXa inhibitor in TG. ${ }^{42}$ Due the protracted shape of the curve, the peak showed 
the highest variation. The variation in effect of both DOACs on TG within twelve healthy individuals remained fairly stable over one year for the most donors, with some exceptions. Interestingly, donors showing a highly variable response to rivaroxaban could have a more stable response to apixaban and vice versa. This suggests that the variability found over time is not only caused by the donor characteristics, which could lead to the hypothesis that, based on the effects in plasma, one DOAC could be more suitable for a given individual than another.

Another remarkable observation was that the donors using hormonal contraceptives had a clearly higher TG, higher prothrombin conversion and a lower response to rivaroxaban and apixaban. It is known that the use of hormonal contraceptives is correlated with a higher risk of thrombosis, previously biochemically characterized as a resistance of TG against the effects of activated protein $\mathrm{C}$ (so called APC resistance). The apparent and quite remarkably reduced activity of DOACs on the TG in plasma from these two females on hormonal contraceptives, raises the question whether in the rare situation that these contraceptives need to be continued in combination with anticoagulation, a DOAC is the ideal anticoagulant as compared to INR-titrated VKA. In general, this study was performed in vitro and solely addressed the pharmacodynamic effects of DOACs in healthy controls, not taking into account pharmacokinetics. It is likely that the pharmacokinetics might induce additional variation, which will superimpose on the variability reported here. It remains unclear at the moment, whether the observed variation in this study correlates with clinical outcomes as bleeding and thrombosis, which remains to be established in further research.

\section{Exploring the differences in DOAC effects on fibrinolysis}

Both classes of DOACs operate via similar mechanisms as small reversible inhibitors, but on different coagulation factors. Dabigatran neutralizes thrombin directly, whereas rivaroxaban, apixaban and edoxaban inhibit FXa, hence the formation of thrombin, i.e. the activation of prothrombin by the prothrombinase complex. The pivotal enzyme thrombin has important functions to protect the clot from fibrinolysis. A prominent way in which DOACs could affect fibrinolysis is by modifying the thrombin-mediated activation of TAFI. In the above, we discussed several differences between the types of DOACs in their alteration of the TG curve. This observation lead us to investigate whether the two types of DOACs could have a different mode of action on fibrinolysis as well, caused by their different effect on thrombin (formation). Chapter 7 describes the in vitro evaluation of the different effects 
of DTI as compared to direct FXa inhibitors on fibrinolysis, studied with turbidimetry and TG. We found that both types of DOACs accelerate fibrinolysis by diminishing thrombin. When TM is added, a catalyst of TAFI activation by thrombin, the clot lysis time (CLT) was prolonged considerably in all conditions, with and without DOACs. We confirmed that this prolongation was TAFI dependent by adding a specific TAFI inhibitor, which is consistent with findings form other groups ${ }^{88,89}$. We studied the CLT in twenty healthy individuals, using plasma spiked with different types of DOACs in comparable concentrations. Interestingly, we found a clear dose-dependent shortening of the CLT in the absence and presence of TM for dabigatran, indicating a profibrinolytic effect in both conditions. This was not the case for rivaroxaban and apixaban, which both showed an accelerated clot lysis in the absence of $\mathrm{TM}$, but a limited effect in its presence. It was shown before that dabigatran, as a small molecule can also inhibit thrombin when incorporated in a complex. ${ }^{44,45}$ Carrying this type of reasoning further, we propose that dabigatran inhibits thrombin bound to TM, thereby reducing TAFI activation and enabling more efficient clot lysis. Direct FXa inhibitors do not affect the thrombin-TM complex. Consequently, TAFI can still be activated which leads to better protection of the clot.

We also report substantial differences between healthy individuals in their response to a fixed dose of DOAC in our experiments with TG. For example, one donor barely responded to increasing fixed doses of DTI (little reduction of ETP), while showing an average response to the comparable doses of both fixed direct FXa inhibitors. Obviously, such data need to be corroborated in larger experimental studies. These experiments were all performed in vitro using spiked plasma from healthy donors. The question remains in which way these in vitro observations will affect the clinical picture. It would be fascinating to know whether dabigatran has indeed a more profibrinolytic profile than direct FXa inhibitors in patients using DOACs. In the large DOAC trials, more myocardial infarctions were seen in the group using dabigatran. Hypothetically, a more profibrinolytic character could lead to a less stable clot and consequently easier embolization. Insight in differential pro- or antifibrinolytic effects between DOACs could ultimately be used to our advantage in the treatment of patients. Theoretically, DOACs with additional profibrinolytic characteristics might improve the efficacy of anticoagulant treatment in preventing postthrombotic syndrome in patients with deep venous thrombosis, or other indications for anticoagulation. 


\section{GENERAL CONCLUSION AND FUTURE PROSPECTS}

Coagulation is a complex system that can be analysed with TG, measuring the (individual) capacity of thrombin formed over time. TG is a functional assay that is associated with the risk of thrombosis and bleeding. This thesis confirms that TG may be helpful in estimating the risk of bleeding in patients treated with VKA. Additional research should explore whether there could be a place for TG in prediction scores for bleeding.

As for DOACs, we have shown that the effect of DTI is not reliably represented by the functional parameters of TG. In contrast, TG is able to measure the effect of all direct FXa inhibitors, but with variable response. In this thesis the development of an assay is described that can detect the amount of rivaroxaban (the drug level) and simultaneously measure the effect of this level on individual coagulation with TG. There is a large variation in DOAC levels in response to a fixed oral dose between and within individuals. From the available literature, there is evidence that extreme DOAC levels are associated with clinical outcomes, i.e. thrombosis or bleeding. However, drug levels do not adequately represent the effect of DOACs on the individual's coagulation cascade, including fibrinolysis.

This thesis also shows that the response to a fixed dose of DOACs is highly variable at an individual level, but is relatively stable within individuals over time. This would suggest that in selected cases, patients using DOACs would benefit from the determination of drug levels combined with TG testing (to obtain more insight in the drug effect). Further research is needed to establish the benefit of functional versus quantitative assays with respect to outcome. These studies should ideally be prospective cohort- or large registry studies measuring DOAC levels and their activity, together with outcomes. These studies should be performed for every DOAC. When TG is considered in these trials, a baseline TG without DOAC should not be omitted. An example of a study following this approach is the paper recently published by Yin et al. ${ }^{90}$ They show that the response to edoxaban by measuring the endogenous activity of FXa is more strongly associated with clinical outcome than DOAC levels.

The large DOAC trails have shown that regular monitoring is not needed to achieve efficacy and safety compared to therapy with dose adjusted VKA. The important question remains whether DOAC therapy could be further improved by laboratory testing of drug levels or 
activity to optimize drug and dose selection in individual patients. In some cases the determination of drug levels will be enough to answer a straightforward clinical questions regarding drug accumulation or to exclude the presence of DOACs. In other situations measuring the activity of a DOAC might be of additional value.

Further research should evaluate whether there is a place for TG in DOAC management in certain niches:

- Frail and/or elderly patients

- Patients with renal or hepatic failure

- Perioperative settings

- To manage antidote administration

- Patients with extreme weight

- Patients using combined antithrombotic therapy

- Patients using combined hormonal contraceptives

Other fields in medicine, like oncology, cardiovascular disease, surgery and recently infectious diseases, are increasingly moving towards more personalized treatment. It may well be that in the future even DOAC therapy will develop from one-size-fits-all to a more personalized selection and dosing of the drug. In this thesis is shown that in vitro some patients responded differently to one DOAC than another. It can be imagined that in the near future functional and potentially also genetic testing will guide us in the optimal use of a given DOAC (or VKA) in the individual patient. 


\section{REFERENCES:}

1. Hellenbart EL, Faulkenberg KD, Finks SW. Evaluation of bleeding in patients receiving direct oral anticoagulants. Vasc Health Risk Manag. 2017;13:325-342.

2. Piazza G, Nguyen TN, Cios D, et al. Anticoagulation-associated adverse drug events. Am J Med. 2011;124(12):1136-1142.

3. Shehab N, Lovegrove MC, Geller Al, Rose KO, Weidle NJ, Budnitz DS. US Emergency Department Visits for Outpatient Adverse Drug Events, 2013-2014. Jama. 2016;316(20):2115-2125.

4. Ten Cate V, Ten Cate H, Verheugt FW. Erratum to: The Global Anticoagulant Registry in the FIELD - Atrial Fibrillation (GARFIELD-AF): Exploring the changes in anticoagulant practice in patients with non-valvular atrial fibrillation in the Netherlands. Neth Heart J. 2016;24(12):768.

5. Heidbuchel H, Verhamme P, Alings M, et al. Updated European Heart Rhythm Association Practical Guide on the use of non-vitamin $\mathrm{K}$ antagonist anticoagulants in patients with non-valvular atrial fibrillation. Europace. 2015;17(10):1467-1507.

6. Weitz JI, Eikelboom JW. Urgent Need to Measure Effects of Direct Oral Anticoagulants. Circulation. 2016;134(3):186-188

7. Levy JH, Ageno W, Chan NC, Crowther M, Verhamme P, Weitz JI. When and how to use antidotes for the reversal of direct oral anticoagulants: guidance from the SSC of the ISTH. J Thromb Haemost. 2016;14(3):623-627.

8. Baglin T, Hillarp A, Tripodi A, Elalamy I, Buller H, Ageno W. Measuring Oral Direct Inhibitors (ODIs) of thrombin and factor Xa: A recommendation from the Subcommittee on Control of Anticoagulation of the Scientific and Standardisation Committee of the International Society on Thrombosis and Haemostasis. J Thromb Haemost. 2013.

9. Hemker HC, Giesen P, AIDieri R, et al. The calibrated automated thrombogram (CAT): a universal routine test for hyper- and hypocoagulability. Pathophysiol Haemost Thromb. 2002;32(5-6):249-253.

10. Hron G, Kollars M, Binder BR, Eichinger S, Kyrle PA. Identification of patients at low risk for recurrent venous thromboembolism by measuring thrombin generation. Jama. 2006;296(4):397-402.

11. Tripodi A, Legnani C, Chantarangkul V, Cosmi B, Palareti G, Mannucci PM. High thrombin generation measured in the presence of thrombomodulin is associated with an increased risk of recurrent venous thromboembolism. J Thromb Haemost. 2008;6(8):1327-1333.

12. Baglin T. Unraveling the thrombophilia paradox: from hypercoagulability to the prothrombotic state. J Thromb Haemost. 2010;8(2):228-233.

13. Dargaud $Y$, Beguin $S$, Lienhart $A$, et al. Evaluation of thrombin generating capacity in plasma from patients with haemophilia A and B. Thromb Haemost. 2005;93(3):475-480.

14. de Koning MLY, Fischer K, de Laat B, Huisman A, Ninivaggi M, Schutgens REG. Comparing thrombin generation in patients with hemophilia A and patients on vitamin K antagonists. J Thromb Haemost. 2017;15(5):868-875.

15. Bosch YP, Al Dieri R, ten Cate $H$, et al. Measurement of thrombin generation intra-operatively and its association with bleeding tendency after cardiac surgery. Thromb Res. 2014;133(3):488-494.

16. Luna-Zaizar H, Gonzalez-Moncada Al, Padilla-Lopez EL, et al. Thrombin generation and international normalized ratio in inherited thrombophilia patients receiving thromboprophylactic therapy. Thromb Res. 2015;136(6):1291-1298.

17. Gatt A, van Veen JJ, Bowyer A, et al. Wide variation in thrombin generation in patients with atrial fibrillation and therapeutic International Normalized Ratio is not due to inflammation. Br J Haematol. 2008;142(6):946-952.

18. Dargaud $Y$, Hoffman $M$, Lefrapper $L$, et al. Bleeding risk in warfarinized patients with a therapeutic international normalized ratio: the effect of low factor IX levels. J Thromb Haemost. 2013;11(6):1043-1052. 
19. Apostolakis S, Lane DA, Buller H, Lip GY. Comparison of the CHADS2, CHA2DS2-VASc and HAS-BLED scores for the prediction of clinically relevant bleeding in anticoagulated patients with atrial fibrillation: the AMADEUS trial. Thromb Haemost. 2013;110(5):1074-1079.

20. Bloemen S, Zwaveling S, Ten Cate H, Ten Cate-Hoek A, de Laat B. Prediction of bleeding risk in patients taking vitamin K antagonists using thrombin generation testing. PLoS One. 2017;12(5):e0176967.

21. Marchetti M, Giaccherini C, Lerede T, Maggioni A, Cefis M, Falanga A. Characterization of thrombin generation (TG) by the calibrated automated thrombography (CAT) in atrial fibrillation (AF) patients on permanent oral anticoagulation therapy (OAT) with warfarin. Journal of Thrombosis and Haemostasis. 2013;3):59-60.

22. Verzeroli C, Giaccherini C, Marchetti M, Milesi V, Barcella L, Falanga A. Thrombin generation (TG) potential in patients on chronic oral anticoagulant therapy (OAT). Thrombosis Research. 2014;2):S98-S99.

23. Choi Q, Kim JE, Hyun J, Han KS, Kim HK. Contributions of procoagulants and anticoagulants to the international normalized ratio and thrombin generation assay in patients treated with warfarin: potential role of protein $\mathrm{Z}$ as a powerful determinant of coagulation assays. Thromb Res. 2013;132(1):e70-75.

24. Herpers R, Van Beem RT, Michel WM, et al. INR vs. Thrombin generation assays for guiding VKA reversal: A retrospective comparison. Nederlands Tijdschrift voor Klinische Chemie en Laboratoriumgeneeskunde. 2015;40 (2):107-108.

25. Helin TA, Lemponen M, Hjemdahl P, Ronquist-Nii Y, Lassila R, Joutsi-Korhonen L. From laboratory to clinical practice: Dabigatran effects on thrombin generation and coagulation in patient samples. Thromb Res. 2015;136(1):154-160.

26. Douxfils J, Mullier F, Robert S, Chatelain C, Chatelain B, Dogne JM. Impact of dabigatran on a large panel of routine or specific coagulation assays. Laboratory recommendations for monitoring of dabigatran etexilate. Thromb Haemost. 2012;107(5):985-997.

27. Bloemen S, Zwaveling S, Douxfils J, Roest M, Kremers R, Mullier F. The anticoagulant effect of dabigatran is reflected in the lag time and time-to-peak, but not in the endogenous thrombin potential or peak, of thrombin generation. Thromb Res. 2018;171:160-166.

28. Wagenvoord RJ, Deinum J, Elg M, Hemker HC. The paradoxical stimulation by a reversible thrombin inhibitor of thrombin generation in plasma measured with thrombinography is caused by alpha-macroglobulin-thrombin J Thromb Haemost. 2010;8(6):1281-1289.

29. Gribkova IV, Lipets EN, Rekhtina IG, et al. The modification of the thrombin generation test for the clinical assessment of dabigatran etexilate efficiency. Sci Rep. 2016;6:29242.

30. Perzborn E, Heitmeier S, Buetehorn U, Laux V. Direct thrombin inhibitors, but not the direct factor Xa inhibitor rivaroxaban, increase tissue factor-induced hypercoagulability in vitro and in vivo. J Thromb Haemost. 2014;12(7):1054-1065

31. Zahir H, Brown KS, Vandell AG, et al. Edoxaban effects on bleeding following punch biopsy and reversal by a 4-factor prothrombin complex concentrate. Circulation. 2015;131(1):82-90.

32. Levi M, Moore KT, Castillejos CF, et al. Comparison of three-factor and four-factor prothrombin complex concentrates regarding reversal of the anticoagulant effects of rivaroxaban in healthy volunteers. $J$ Thromb Haemost. 2014;12(9):1428-1436.

33. Herrmann R, Thom J, Wood A, Phillips M, Muhammad S, Baker R. Thrombin generation using the calibrated automated thrombinoscope to assess reversibility of dabigatran and rivaroxaban. Thromb Haemost. 2014;111(5):989-995.

34. Perzborn E, Heitmeier S, Laux V, Buchmuller A. Reversal of rivaroxaban-induced anticoagulation with prothrombin complex concentrate, activated prothrombin complex concentrate and recombinant activated factor VII in vitro. Thromb Res. 2014;133(4):671-681. 
35. Marlu R, Hodaj E, Paris A, Albaladejo P, Cracowski JL, Pernod G. Effect of non-specific reversal agents on anticoagulant activity of dabigatran and rivaroxaban: a randomised crossover ex vivo study in healthy volunteers. Thromb Haemost. 2012;108(2):217-224.

36. Dinkelaar J, Patiwael S, Harenberg J, Leyte A, Brinkman HJ. Global coagulation tests: their applicability for measuring direct factor $\mathrm{Xa}$ - and thrombin inhibition and reversal of anticoagulation by prothrombin complex concentrate. Clin Chem Lab Med. 2014;52(11):1615-1623.

37. Eerenberg ES, Kamphuisen PW, Sijpkens MK, Meijers JC, Buller HR, Levi M. Reversal of rivaroxaban and dabigatran by prothrombin complex concentrate: a randomized, placebo-controlled, crossover study in healthy subjects. Circulation. 2011;124(14):1573-1579.

38. Cheung KC, Rodgers S, McRae S. Local experience in reversal dabigatran using activated prothrombin complex concentrates (FEIBA). Haematologica. 2012;97:185.

39. Gatt A, van Veen JJ, Woolley AM, Kitchen S, Cooper P, Makris M. Thrombin generation assays are superior to traditional tests in assessing anticoagulation reversal in vitro. Thromb Haemost. 2008;100(2):350-355.

40. Chan NC, Coppens M, Hirsh J, et al. Real-world variability in dabigatran levels in patients with atrial fibrillation. J Thromb Haemost. 2015;13(3):353-359.

41. Testa S, Tripodi A, Legnani C, et al. Plasma levels of direct oral anticoagulants in real life patients with atrial fibrillation: Results observed in four anticoagulation clinics. Thromb Res. 2016;137:178-183.

42. Bloemen S, Hemker HC, Al Dieri R. Large inter-individual variation of the pharmacodynamic effect of anticoagulant drugs on thrombin generation. Haematologica. 2013;98(4):549-554.

43. Giesen PLA, Gulpen AJW, van Oerle R, ten Cate H, Spronk HM. Calibrated Automated Thrombogram II: A new thrombin generation test able to measure in the presence of a direct thrombin inhibitor. Research and Practice in Thrombosis and Haemostasis 2017.

44. Cunanan J. Effect Of Dabigatran and Rivaroxiban On Thrombomodulin Mediated Activation Of Protein $\mathrm{C}$ and Thrombin Activated Fibrinolysis Inhibitor (TAFI). Potential Clinical Implications. Blood. 2013;122(21):3641.

45. Wienen W, Stassen JM, Priepke H, Ries UJ, Hauel N. In-vitro profile and ex-vivo anticoagulant activity of the direct thrombin inhibitor dabigatran and its orally active prodrug, dabigatran etexilate. Thromb Haemost. 2007;98(1):155-162.

46. Rigano J, Ng C, Nandurkar H, Ho P. Thrombin generation estimates the anticoagulation effect of direct oral anticoagulants with significant interindividual variability observed. Blood Coagul Fibrinolysis. 2018;29(2):148-154.

47. De Smedt E, Al Dieri R, Spronk HM, Hamulyak K, ten Cate H, Hemker HC. The technique of measuring thrombin generation with fluorogenic substrates: 1. Necessity of adequate calibration. Thromb Haemost. 2008;100(2):343-349.

48. Schiele F, van Ryn J, Canada K, et al. A specific antidote for dabigatran: functional and structural characterization. Blood. 2013;121(18):3554-3562.

49. Pollack CV, Jr., Reilly PA, van Ryn J, et al. Idarucizumab for Dabigatran Reversal - Full Cohort Analysis. N Engl J Med. 2017;377(5):431-441.

50. Beguin S, Kessels H, Dol F, Hemker HC. The consumption of antithrombin III during coagulation, its consequences for the calculation of prothrombinase activity and the standardisation of heparin activity. Thromb Haemost. 1992;68(2):136-142.

51. Kremers RM, Peters TC, Wagenvoord RJ, Hemker HC. The balance of pro- and anticoagulant processes underlying thrombin generation. J Thromb Haemost. 2015;13(3):437-447.

52. Robert S, Ghiotto J, Pirotte B, et al. Is thrombin generation the new rapid, reliable and relevant pharmacological tool for the development of anticoagulant drugs? Pharmacol Res. 2009;59(3):160-166.

53. al Dieri R, Alban S, Beguin S, Hemker HC. Thrombin generation for the control of heparin treatment, comparison with the activated partial thromboplastin time. J Thromb Haemost. 2004;2(8):1395-1401. 
54. Douxfils J, Chatelain C, Chatelain B, Dogne JM, Mullier F. Impact of apixaban on routine and specific coagulation assays: a practical laboratory guide. Thromb Haemost. 2013;110(2):283-294.

55. Samama MM, Mendell J, Guinet C, Le Flem L, Kunitada S. In vitro study of the anticoagulant effects of edoxaban and its effect on thrombin generation in comparison to fondaparinux. Thromb Res. 2012;129(4):e77-82.

56. Douxfils J, Mullier F, Loosen C, Chatelain C, Chatelain B, Dogne JM. Assessment of the impact of rivaroxaban on coagulation assays: laboratory recommendations for the monitoring of rivaroxaban and review of the literature. Thromb Res. 2012;130(6):956-966.

57. Bloemen S. Managing anticoagulant therapies using innovative thrombin generation assays. 2014

58. Kim PY, Yeh CH, Dale BJ, et al. Mechanistic Basis for the Differential Effects of Rivaroxaban and Apixaban on Global Tests of Coagulation. TH Open. 2018;2:190-201.

59. Tripodi A, Ageno W, Ciaccio M, et al. Position Paper on laboratory testing for patients on direct oral anticoagulants. A Consensus Document from the SISET, FCSA, SIBioC and SIPMeL. Blood Transfus. 2018;16(5):462-470.

60. Kitchen S, Gray E, Mackie I, Baglin T, Makris M, committee B. Measurement of non-coumarin anticoagulants and their effects on tests of Haemostasis: Guidance from the British Committee for Standards in Haematology. Br J Haematol. 2014;166(6):830-841.

61. Samuelson BT, Cuker A. Measurement and reversal of the direct oral anticoagulants. Blood Rev. 2016.

62. Tripodi A. To measure or not to measure direct oral anticoagulants before surgery or invasive procedures. $J$ Thromb Haemost. 2016;14(7):1325-1327.

63. Avecilla ST, Ferrell C, Chandler WL, Reyes M. Plasma-diluted thrombin time to measure dabigatran concentrations during dabigatran etexilate therapy. Am J Clin Pathol. 2012;137(4):572-574.

64. Cuker A, Siegal DM, Crowther MA, Garcia DA. Laboratory measurement of the anticoagulant activity of the non-vitamin K oral anticoagulants. J Am Coll Cardiol. 2014;64(11):1128-1139.

65. Douxfils J, Chatelain B, Hjemdahl P, et al. Does the Russell Viper Venom time test provide a rapid estimation of the intensity of oral anticoagulation? A cohort study. Thromb Res. 2015;135(5):852-860.

66. Douxfils J, Dogne JM, Mullier F, et al. Comparison of calibrated dilute thrombin time and aPTT tests with LC-MS/MS for the therapeutic monitoring of patients treated with dabigatran etexilate. Thromb Haemost. 2013;110(3):543-549

67. Douxfils J, Tamigniau A, Chatelain B, et al. Comparison of calibrated chromogenic anti-Xa assay and PT tests with LC-MS/MS for the therapeutic monitoring of patients treated with rivaroxaban. Thromb Haemost. 2013;110(4):723-731.

68. Douxfils J, Ageno W, Samama CM, et al. Laboratory testing in patients treated with direct oral anticoagulants: a practical guide for clinicians. J Thromb Haemost. 2018;16(2):209-219.

69. Godier A, Dincq AS, Martin AC, et al. Predictors of pre-procedural concentrations of direct oral anticoagulants: a prospective multicentre study. Eur Heart J. 2017;38(31):2431-2439.

70. Al-Aieshy F, Malmstrom RE, Antovic J, et al. Clinical evaluation of laboratory methods to monitor exposure of rivaroxaban at trough and peak in patients with atrial fibrillation. Eur J Clin Pharmacol. 2016;72(6):671-679.

71. Albaladejo P, Samama CM, Sie P, et al. Management of Severe Bleeding in Patients Treated with Direct Oral Anticoagulants: An Observational Registry Analysis. Anesthesiology. 2017;127(1):111-120.

72. Ten Cate H, Olie RH, Ten Cate-Hoek AJ, Henskens YMC. Direct oral anticoagulants: When to consider laboratory testing? Int J Lab Hematol. 2018;40 Suppl 1:30-33.

73. Testa S, Paoletti O, Legnani C, et al. Low drug levels and thrombotic complications in high-risk atrial fibrillation patients treated with direct oral anticoagulants. J Thromb Haemost. 2018;16(5):842-848.

74. Reilly PA, Lehr T, Haertter S, et al. The effect of dabigatran plasma concentrations and patient characteristics on the frequency of ischemic stroke and major bleeding in atrial fibrillation patients: the RE-LY Trial (Randomized Evaluation of Long-Term Anticoagulation Therapy). J Am Coll Cardiol. 2014;63(4):321-328. 
75. Giugliano RP, Ruff CT, Braunwald E, et al. Edoxaban versus warfarin in patients with atrial fibrillation. N Engl J Med. 2013;369(22):2093-2104.

76. Perzborn E, Kubitza D, Misselwitz F. Rivaroxaban. A novel, oral, direct factor Xa inhibitor in clinical development for the prevention and treatment of thromboembolic disorders. Hamostaseologie. 2007;27(4):282-289.

77. Stangier J. Clinical pharmacokinetics and pharmacodynamics of the oral direct thrombin inhibitor dabigatran etexilate. Clinical pharmacokinetics. 2008;47(5):285-295.

78. Raghavan N, Frost CE, Yu Z, et al. Apixaban Metabolism and Pharmacokinetics after Oral Administration to Humans. Drug Metabolism and Disposition. 2009;37(1):74-81.

79. Steffel J, Verhamme P, Potpara TS, et al. The 2018 European Heart Rhythm Association Practical Guide on the use of non-vitamin $\mathrm{K}$ antagonist oral anticoagulants in patients with atrial fibrillation: executive summary. Europace. 2018.

80. Samama MM, Guinet C, Le Flem L, Ninin E, Debue JM. Measurement of dabigatran and rivaroxaban in primary prevention of venous thromboembolism in 106 patients, who have undergone major orthopedic surgery: an observational study. J Thromb Thrombolysis. 2013;35(2):140-146.

81. Gong IY, Kim RB. Importance of pharmacokinetic profile and variability as determinants of dose and response to dabigatran, rivaroxaban, and apixaban. Can J Cardiol. 2013;29(7 Suppl):S24-33.

82. Kubitza D, Roth A, Becka M, et al. Effect of hepatic impairment on the pharmacokinetics and pharmacodynamics of a single dose of rivaroxaban, an oral, direct Factor Xa inhibitor. Br J Clin Pharmacol. 2013;76(1):89-98.

83. Samuelson BT, Cuker A. Measurement and reversal of the direct oral anticoagulants. Blood Rev. 2017;31(1):77-84.

84. Hawes EM, Deal AM, Funk-Adcock D, et al. Performance of coagulation tests in patients on therapeutic doses of dabigatran: a cross-sectional pharmacodynamic study based on peak and trough plasma levels. J Thromb Haemost. 2013;11(8):1493-1502.

85. Francart SJ, Hawes EM, Deal AM, et al. Performance of coagulation tests in patients on therapeutic doses of rivaroxaban. A cross-sectional pharmacodynamic study based on peak and trough plasma levels. Thromb Haemost. 2014;111(6):1133-1140.

86. Testa S, Tripodi A, Legnani C, et al. Plasma levels of direct oral anticoagulants in real life patients with atrial fibrillation: Results observed in four anticoagulation clinics. Thromb Res. 2016;137:178-183.

87. Eikelboom JW, Quinlan DJ, Hirsh J, Connolly SJ, Weitz JI. Laboratory Monitoring of Non-Vitamin K Antagonist Oral Anticoagulant Use in Patients With Atrial Fibrillation: A Review. JAMA Cardiol. 2017;2(5):566-574.

88. Ammollo CT, Semeraro F, Incampo F, Semeraro N, Colucci M. Dabigatran enhances clot susceptibility to fibrinolysis by mechanisms dependent on and independent of thrombin-activatable fibrinolysis inhibitor. J Thromb Haemost. 2010;8(4):790-798.

89. Semeraro F, Incampo F, Ammollo CT, et al. Dabigatran but not rivaroxaban or apixaban treatment decreases fibrinolytic resistance in patients with atrial fibrillation. Thromb Res. 2016;138:22-29.

90. Yin OQP, Antman EM, Braunwald E, et al. Linking Endogenous Factor Xa Activity, a Biologically Relevant Pharmacodynamic Marker, to Edoxaban Plasma Concentrations and Clinical Outcomes in the ENGAGE AF-TIMI 48 Trial. Circulation. 2018;138(18):1963-1973. 


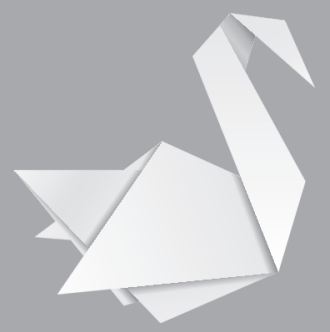




\section{CHAPTER \\ Nederlandse \\ samenvatting

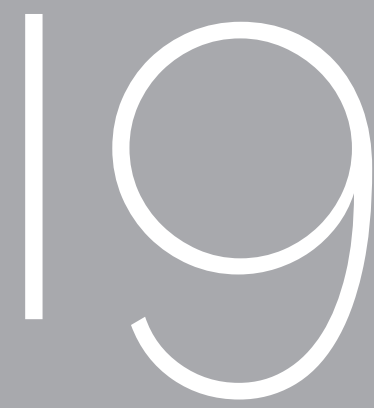




\section{NEDERLANDSE SAMENVATTING}

In deze thesis hebben we de effecten van orale antistollingsmedicatie (anticoagulantia) op de bloedstolling en op de afbraak van bloedstolsels (fibrinolyse), onderzocht.

Hoofdstuk 1 is de inleiding van dit proefschrift. In dit hoofdstuk wordt de fysiologie van de vorming en de afbraak van een stolsel uitgelegd. Daarna wordt ingegaan op enkele belangrijke aandoeningen waarin de bloedstolling verstoord is en wordt de huidige behandeling hiervan besproken. Trombose (ongewenste stolling) is een veel voorkomende aandoening met een hoge co-morbiditeit en mortaliteit. In Nederland gebruiken ongeveer 1,5 miljoen mensen anticoagulantia ter bescherming, of als behandeling, tegen trombose. De meest voorkomende bijwerking van deze medicijnen zijn bloedingen. Gebruikers van anticoagulantia hebben ongeveer $10-17 \%$ kans per behandeljaar om een bloeding te krijgen, waarvoor medische hulp noodzakelijk is. ${ }^{1}$ Het adequaat instellen van antistolling is dus van groot belang. In Nederland wordt de zorg omtrent orale anticoagulantia geregeld via trombosediensten. In deze gespecialiseerde centra wordt het effect van de medicatie gecontroleerd met behulp van een stollingstest: de protrombine tijd (PT). Voor vitamine $\mathrm{K}$ antagonisten (VKA's), die tot voor kort de meest gebruikte orale anticoagulantia waren, wordt met behulp van deze test de juiste, persoonlijke dosis bepaald.

In het afgelopen decennium hebben vier nieuwe, orale anticoagulantia, "direct werkende orale anticoagulantia"(DOACs) hun intrede gemaakt in de kliniek. Deze middelen remmen allen slechts één stollingsfactor, in tegenstelling tot de VKA's die de vorming van vier stollingsfactoren belemmeren. Dabigatran is een directe remmer van trombine (factor Ila). Rivaroxaban, apixaban en edoxaban remmen factor Xa. Mede vanwege deze selectieve remming wordt gezegd dat ze stabieler zijn qua farmacokinetiek en -dynamiek dan de VKA's. Grote, gerandomiseerde studies hebben de efficiëntie en veiligheid van DOACs onderzocht om veneuze trombose te voorkomen en te behandelen.2-9 De auteurs van deze studies concludeerden dat DOACs in een vaste dosering kunnen worden voorgeschreven en dat (regelmatige) controle van de stolling gedurende de behandeling niet nodig is. Hierbij lijkt het gebied trombose en hemostase dus af te wijken van andere gebieden in de geneeskunde, waar behandeling meer en meer wordt gepersonaliseerd ('personalized medicine' in tegenstelling tot 'one-size-fits-all'). 
Hoewel het routinematige monitoren van DOACs wordt afgeraden door richtlijnen is er wel een toenemende consensus dat het meten van (het effect van) DOACs in sommige situaties nuttig kan zijn. ${ }^{10-13}$ Het meten van het effect van deze middelen op de stolling is een uitdaging. Traditionele stollingstesten, zoals de PT en de aPTT, die respectievelijk gebruikt worden voor het meten van VKA's en heparines, geven een minder betrouwbare weergave van de effecten van DOACs. Er zijn wel speciale testen ontwikkeld om plasmaconcentraties van DOACs te meten. Deze testen zijn gevoelig voor de aanwezigheid van DOACs, maar zeggen weinig over het effect van deze DOACs op de stolling. In dit proefschrift wordt de trombinegeneratie (TG) test onderzocht als potentiele test om het effect van DOACs op de bloedstolling te meten. Deze test meet de hoeveelheid trombine die gevormd wordt over tijd. Daarbij kunnen onder bepaalde condities de extra feedbackloops van de stollingscascade ook gemeten worden. TG wordt daarom gezien als een functionele test die de globale stolling weergeeft, in tegenstelling tot slechts het eerste gedeelte (zoals bij de traditionele stollingstesten). Bovendien wijst eerder onderzoek uit dat TG het effect van meerdere soorten anticoagulantia op stolling kan weergeven. ${ }^{14,15}$

In hoofdstuk 2 werd onderzocht of trombinegeneratie patiënten ( $n=129)$ met een verhoogd risico op bloedingen kan identificeren tijdens de behandeling met VKA. Trombinegeneratie werd gemeten met CAT (calibrated automated thrombinography) in plaatjesarm plasma (PPP), plaatjesrijk plasma (PRP) en in volbloed, met en zonder de toevoeging van trombomoduline (TM). Daarnaast werd voor elke patiënt de HASBLED-score uitgerekend (i.e. een bloedingsscore). In volbloed CAT bleek dat "endogene trombinepotentiaal" (ETP) en de peak-waarde bloedingen even goed konden voorspellen als de HAS-BLED score. Dit impliceert dat het zinvol zou kunnen zijn om de volbloed CAT te implementeren in HASBLED om zo de voorspellende waarde te vergroten. In PPP zagen we een trend maar geen significante daling in de ETP en peak bij patiënten met een bloeding, in tegenstelling tot eerdere onderzoeken. ${ }^{16,17}$ Het toevoegen van TM, wat de test gevoeliger zou moeten maken, veranderde niets aan deze resultaten.

In hoofdstuk 3 hebben we het verband tussen een verlaagde TG en het risico op bloedingen in patiënten op anticoagulantia verder onderzocht in een systematische literatuurstudie. Hoewel CAT vaak gebruikt wordt om de effecten van antistolling te onderzoeken in verschillende onderzoeksinstellingen, zijn er maar weinig klinische studies gedaan naar de directe relatie tussen TG en bloedingen in patiënten die behandeld worden met orale antistolling. Alle 
artikelen die aan de inclusiecriteria voldeden ( $n=7)$, onderzochten patiënten die behandeld werden met VKA. De twee studies met de hoogste methodologische kwaliteit vonden beiden aanwijzingen dat er een verband bestaat tussen lage TG-waarden en bloedingen. ${ }^{16,18}$ De andere studies, slechts van matige kwaliteit, sloten aan bij deze bevindingen, maar hadden zelf niet genoeg solide data om deze hypothese te kunnen valideren.

De remming van stolling door anticoagulantia is zichtbaar in het TG experiment doordat de TG-curve lager wordt en later van start gaat, hetgeen een teken is van een verminderde capaciteit om trombine te vormen. Klassiek wordt de tijd tot de vorming van het eerste trombine langer (lag time); wordt de tijd tot de maximale hoeveelheid trombine gevormd is langer (time-to-peak) en neemt de hoeveelheid gevormde trombine over tijd af (ETP), evenals de maximale hoeveelheid trombine (peak). ${ }^{14,15}$ Wanneer dabigatran wordt toegevoegd aan het TG experiment, worden de lag time en de time-to-peak langer (zoals verwacht) maar worden de ETP en peak hoger (tegen de verwachting in). Dit zou kunnen leiden tot de foutieve conclusie dat de toevoeging van een reversibele trombineremmer de vorming van trombine zou stimuleren. In Hoofdstuk 4 wordt het paradoxale effect van de directe trombineremmer (dabigatran) op CAT in meer detail behandeld. Er worden twee mechanismes aangetoond die verantwoordelijk zijn voor het artefact in TG:

1. Het grootste gedeelte van de verhoging van de TG curve komt door een artefact als gevolg van de calibratie. In CAT wordt een vaste concentratie $\mathrm{a}_{2}$-macroglobuline $\left(a_{2} M\right)$-trombine als calibrator gebruikt. Hoewel trombine gebonden is aan $a_{2} M$, kan deze nog steeds geremd worden door dabigatran. Wanneer dabigatran dus aanwezig is in de calibrator well, resulteert dit in een overschatting van de berekende hoeveelheid vrij trombine in de TG-well. We tonen vervolgens aan dat dit effect kan worden opgeheven door de toevoeging van idarucizumab (een antilichaam specifiek gericht tegen dabigatran) aan de calibrator well. Dit corrigeerde echter slechts een gedeelte van de verhoging van de ETP en peak, wat duidt op een tweede artefact.

2. Tijdens het TG experiment wordt een gedeelte van de nieuwe gevormde trombine weggevangen door binding aan $\mathrm{a}_{2} \mathrm{M}$ (20-25\%); trombine gebonden in dit complex kan nog steeds fluorescent substraat knippen en daardoor de indruk wekken dat er nog steeds vrij trombine wordt gevormd. Hiervoor wordt in de berekening van de vrije trombineconcentratie gecorrigeerd door de hoeveelheid $\mathrm{a}_{2} \mathrm{M}$-trombine af te trekken 
van de totale hoeveelheid trombine. Omdat $\mathrm{a}_{2} \mathrm{M}$-trombine dus ook kan worden geremd door dabigatran, wordt de hoeveelheid vrij trombine overschat in de berekening. Dit artefact kan niet gecorrigeerd worden door idarucizumab toe te voegen, omdat dit het gehele effect van dabigatran zal opheffen. Je meet in dit geval dan de ongeremde stolling van de patiënt (dus alsof hij/zij geen dabigatran zou gebruiken).

De opinie dat het meten van DOACs in bepaalde klinische situaties, de behandeling kan verbeteren voor sommige patiënten, heeft de afgelopen jaren steeds meer bijval gekregen..$^{10-13,19}$ Het is van belang dat hierin een onderscheid gemaakt wordt in het meten van DOAC spiegels in het plasma (de concentratie) of het effect van deze spiegels op de stolling zelf. Het is bekend dat de variatie van spiegels binnen en tussen personen erg veel verschilt, wat heeft geleid tot brede "normaalwaardes". Toch zijn er ook studies die een verband hebben gevonden tussen de hoogte van de spiegels en klinische uitkomsten, zoals trombose of bloedingen. ${ }^{4,20,21}$ Meer onderzoek is nodig om te bepalen hoe sterk deze relatie is. Ons leek het interessant om naar zowel de spiegels als het effect op stolling te kijken.

Hoofdstuk 5 beschrijft de ontwikkeling van een test die zowel de spiegels van rivaroxaban kan meten, alsook het effect op stolling, door gebruik te maken van dezelfde CAT-setup: de Xross-CAT. Een goede correlatie werd gevonden tussen de rivaroxaban spiegels gemeten met de Xross-CAT en de Biophen DiXal (een chromogene, gecalibreerde anti-FXa test die nu in de kliniek wordt gebruikt). De correlatie met de PT en de "Diluted Russel Viper Venom time" was matig, en komt overeen met de gevonden relatie tussen andere kwantitatieve en stollingstijd-testen. ${ }^{22}$

De relatie tussen de Xross-CAT spiegels en TG waarde was hyperbool, wat betekent dat kleine hoeveelheden rivaroxaban in staat zijn de vorming van trombine sterk te remmen. Daarnaast laat dit zien dat hogere concentraties rivaroxaban de ETP en peak relatief weinig beïnvloeden, doordat een plateaufase wordt bereikt. Het non-lineaire verband tussen plasmaconcentraties met ETP en peak wijst erop dat deze testparameters informatie geven over verschillende aspecten. Grotere cohort en observationele studies zijn nodig om te evalueren of er een voordeel is om naast concentraties ook het effect op TG te meten. 
Dat de variatie tussen DOAC spiegels tussen patiënten groot is, is reeds bekend. ${ }^{21,23-27}$ De variatie van DOAC concentraties binnen patiënten over tijd is nog weinig onderzocht.

In Hoofdstuk 6 rapporteren we de variatie in het effect van een vaste concentratie rivaroxaban en apixaban (in vitro) op TG tussen gezonde personen (inter-individuele variatie). Daarnaast hebben we gekeken naar de variatie in het effect binnen 12 gezonde personen over de periode van een jaar (intra-individuele variatie). Onze data bevestigen de eerder gevonden grote inter-individuele variatie van een vaste concentratie FXa remmers op TG. ${ }^{14}$ Het intra-individuele effect van FXa remmers op TG bleef vrij stabiel voor de meeste donoren, maar er waren enkele uitzonderingen. Interessant was dat donoren die het meeste variatie toonden bij de ene DOAC (bv. rivaroxaban), niet per definitie het meeste variatie vertoonden bij de andere DOAC (apixaban), of vice versa. Een andere bijzondere observatie was dat twee gezonde vrijwilligers over tijd een sterk verhoogde TG bleven hebben in vergelijking met de andere donoren. De respons van deze donoren op DOACs bleek ook verlaagd ten opzichte van de anderen. Deze donoren waren de enige gebruikers van hormonale anticonceptie. Aangezien deze studie volledig berust op farmacodynamische bevindingen, zal de variatie in effect op TG in vivo waarschijnlijk nog toenemen door de bijkomende farmacokinetische variatie. Verder onderzoek zal deze hypothese en de mogelijke consequenties van de variatie moeten uitwijzen.

In Hoodstuk 7 zijn de in vitro effecten van een directe trombineremmer en directe FXa remmers op fibrinolyse onderzocht met behulp van turbiditeitsmetingen. Beide typen DOACs versnellen fibrinolyse door het (direct of indirect) remmen van trombine. Wanneer TM wordt toegevoegd, een katalysator van de activatie van Thrombin Activatable Fibrinolyis Inhibitor (TAFI) door trombine, duurt het langer voordat een stolsel wordt afgebroken (de afbraaktijd verlengt) zowel in de aan- en afwezigheid van DOACs.

Verder is het verschil in afbraaktijd van een stolsel tussen DOACs bestudeerd in twintig gezonde donoren, door plasma te spiken met een vaste concentratie dabigatran, apixaban en rivaroxaban. Er werd een duidelijke concentratie-afhankelijke versnelling van de afbraaktijd gevonden in de aan- en afwezigheid van TM voor dabigatran. Bij de direct FXa-remmers werd deze versnelde stolselafbraak enkel gezien in de afwezigheid van TM; in de aanwezigheid van TM was dit effect minimaal. Eerder is aangetoond dat dabigatran, als klein molecuul, trombine gebonden in een complex nog steeds kan remmen, doordat het actieve centrum 
in trombine beschikbaar blijft. Volgens deze redenering zou dabigatran trombine kunnen remmen, ook al is het gebonden aan TM. Hierdoor zal minder TAFI door trombine kunnen worden geactiveerd, wat kan leiden tot een efficiëntere stolselafbraak. Trombine in complex met TM zal niet worden beïnvloed door directe FXa remmers, waardoor TAFI kan worden geactiveerd, hetgeen resulteert in een betere bescherming van het stolsel.

Tot slot worden in Hoofdstuk 8 de belangrijkste conclusies samengevat en besproken. Deze thesis bevestigt dat TG een mogelijk hulpmiddel is om het bloedingsrisico in te schatten van patiënten die VKA gebruiken. Aanvullend onderzoek zal moeten aantonen of TG een plaats zou kunnen hebben in risicoscores.

We hebben aangetoond dat het effect van directe trombineremmers niet accuraat wordt weergegeven in de functionele parameters van TG, maar wel in de tijdsafhankelijke parameters. Het effect van verschillende directe FXa remmers wordt wel weergegeven in TG, hetzij met variabele effecten. In deze thesis wordt de ontwikkeling van een test beschreven waarbij zowel de concentratie van rivaroxaban kan worden gemeten, als het effect op stolling met TG. Verder tonen we aan dat het effect van een vaste concentratie DOAC op TG, sterk varieert tussen personen, maar redelijk stabiel blijft binnen een persoon in het verloop van de tijd. Dit houdt in dat in selecte groepen patiënten die DOACs gebruiken, wellicht sommigen baat hebben bij een bepaling van zowel de DOAC concentratie als het effect op TG. Verder onderzoek is nodig om een eventueel voordeel van functionele test versus een kwantitatieve test aan te tonen. 


\section{REFERENTIES}

1. Hellenbart EL, Faulkenberg KD, Finks SW. Evaluation of bleeding in patients receiving direct oral anticoagulants. Vascular health and risk management. 2017;13:325-342.

2. Connolly SJ, Ezekowitz MD, Yusuf S, et al. Dabigatran versus warfarin in patients with atrial fibrillation. The New England journal of medicine. 2009;361(12):1139-1151.

3. Patel MR, Mahaffey KW, Garg J, et al. Rivaroxaban versus warfarin in nonvalvular atrial fibrillation. The New England journal of medicine. 2011;365(10):883-891.

4. Giugliano RP, Ruff CT, Braunwald E, et al. Edoxaban versus warfarin in patients with atrial fibrillation. The New England journal of medicine. 2013;369(22):2093-2104.

5. Granger CB, Alexander JH, McMurray JJ, et al. Apixaban versus warfarin in patients with atrial fibrillation. The New England journal of medicine. 2011;365(11):981-992.

6. Schulman S, Kearon C, Kakkar AK, et al. Dabigatran versus warfarin in the treatment of acute venous thromboembolism. The New England journal of medicine. 2009;361(24):2342-2352.

7. Bauersachs R, Berkowitz SD, Brenner B, et al. Oral rivaroxaban for symptomatic venous thromboembolism. The New England journal of medicine. 2010;363(26):2499-2510.

8. Agnelli G, Buller HR, Cohen A, et al. Oral apixaban for the treatment of acute venous thromboembolism. The New England journal of medicine. 2013;369(9):799-808.

9. Buller HR, Decousus $H$, Grosso MA, et al. Edoxaban versus warfarin for the treatment of symptomatic venous thromboembolism. The New England journal of medicine. 2013;369(15):1406-1415.

10. Heidbuchel H, Verhamme P, Alings M, et al. Updated European Heart Rhythm Association Practical Guide on the use of non-vitamin $\mathrm{K}$ antagonist anticoagulants in patients with non-valvular atrial fibrillation. Europace : European pacing, arrhythmias, and cardiac electrophysiology : journal of the working groups on cardiac pacing, arrhythmias, and cardiac cellular electrophysiology of the European Society of Cardiology. 2015;17(10):1467-1507.

11. Weitz JI, Eikelboom JW. Urgent Need to Measure Effects of Direct Oral Anticoagulants. Circulation. 2016;134(3):186-188.

12. Levy JH, Ageno W, Chan NC, Crowther M, Verhamme P, Weitz JI. When and how to use antidotes for the reversal of direct oral anticoagulants: guidance from the SSC of the ISTH. Journal of thrombosis and haemostasis JTH. 2016;14(3):623-627.

13. Baglin T, Hillarp A, Tripodi A, Elalamy I, Buller H, Ageno W. Measuring Oral Direct Inhibitors (ODIs) of thrombin and factor Xa: A recommendation from the Subcommittee on Control of Anticoagulation of the Scientific and Standardisation Committee of the International Society on Thrombosis and Haemostasis. Journal of thrombosis and haemostasis : JTH. 2013.

14. Bloemen S, Hemker HC, Al Dieri R. Large inter-individual variation of the pharmacodynamic effect of anticoagulant drugs on thrombin generation. Haematologica. 2013;98(4):549-554.

15. Gatt A, van Veen JJ, Woolley AM, Kitchen S, Cooper P, Makris M. Thrombin generation assays are superior to traditional tests in assessing anticoagulation reversal in vitro. Thrombosis and haemostasis. 2008;100(2):350-355.

16. Dargaud $Y$, Hoffman $M$, Lefrapper $L$, et al. Bleeding risk in warfarinized patients with a therapeutic international normalized ratio: the effect of low factor IX levels. Journal of thrombosis and haemostasis : JTH. 2013;11(6):1043-1052.

17. Bosch YP, Al Dieri R, ten Cate $\mathrm{H}$, et al. Measurement of thrombin generation intra-operatively and its association with bleeding tendency after cardiac surgery. Thrombosis research. 2014;133(3):488-494

18. Bloemen S, Zwaveling S, Ten Cate H, Ten Cate-Hoek A, de Laat B. Prediction of bleeding risk in patients taking vitamin $\mathrm{K}$ antagonists using thrombin generation testing. PloS one. 2017;12(5):e0176967. 
19. Tripodi A, Ageno W, Ciaccio M, et al. Position Paper on laboratory testing for patients on direct oral anticoagulants. A Consensus Document from the SISET, FCSA, SIBioC and SIPMeL. Blood transfusion = Trasfusione del sangue. 2018;16(5):462-470.

20. Testa S, Paoletti O, Legnani C, et al. Low drug levels and thrombotic complications in high-risk atrial fibrillation patients treated with direct oral anticoagulants. Journal of thrombosis and haemostasis: JTH. 2018;16(5):842-848.

21. Reilly PA, Lehr T, Haertter $S$, et al. The effect of dabigatran plasma concentrations and patient characteristics on the frequency of ischemic stroke and major bleeding in atrial fibrillation patients: the RE-LY Trial (Randomized Evaluation of Long-Term Anticoagulation Therapy). Journal of the American College of Cardiology. 2014;63(4):321-328.

22. Douxfils J, Tamigniau A, Chatelain B, et al. Comparison of calibrated chromogenic anti-Xa assay and PT tests with LC-MS/MS for the therapeutic monitoring of patients treated with rivaroxaban. Thrombosis and haemostasis. 2013;110(4):723-731.

23. Testa S, Tripodi A, Legnani C, et al. Plasma levels of direct oral anticoagulants in real life patients with atrial fibrillation: Results observed in four anticoagulation clinics. Thrombosis research. 2016;137:178-183.

24. Chan NC, Coppens M, Hirsh J, et al. Real-world variability in dabigatran levels in patients with atrial fibrillation. Journal of thrombosis and haemostasis : JTH. 2015;13(3):353-359.

25. Samama MM, Guinet C, Le Flem L, Ninin E, Debue JM. Measurement of dabigatran and rivaroxaban in primary prevention of venous thromboembolism in 106 patients, who have undergone major orthopedic surgery: an observational study. Journal of thrombosis and thrombolysis. 2013;35(2):140-146.

26. Gong IY, Kim RB. Importance of pharmacokinetic profile and variability as determinants of dose and response to dabigatran, rivaroxaban, and apixaban. Can J Cardiol. 2013;29(7 Suppl):S24-33.

27. Kubitza D, Roth A, Becka M, et al. Effect of hepatic impairment on the pharmacokinetics and pharmacodynamics of a single dose of rivaroxaban, an oral, direct Factor Xa inhibitor. Br J Clin Pharmacol. 2013;76(1):89-98. 


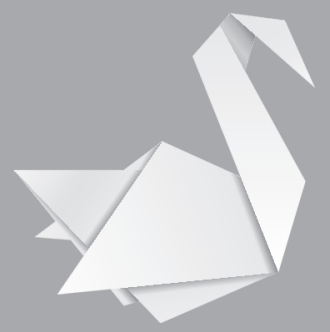


APPENDICES 


\section{VALORISATION}

Venous thrombosis, coronary artery disease and stroke are among the most prevalent cardiovascular diseases in the western population, and thus pose a significant healthcare problem to our society. According to the Dutch Federation of Anticoagulation clinics, about 1.5 million people in the Netherlands are using anticoagulant drugs for prevention or treatment of thrombosis. Although treatment will decrease the individual's risk of (recurrent) thrombosis, it will inevitably increase the risk of bleeding. The balance between the risk of bleeding and thrombosis is crucial in adequate anticoagulant treatment. Vitamin $\mathrm{K}$ antagonists (VKAs) have dominated prevention and treatment of thrombosis for over half a century. These drugs are administered and controlled by specialized anticoagulation clinics. The effect of VKAs is influenced by food and co-medication; therefore, treatment with VKAs requires regular monitoring of the effect using a prothrombin time test. Based on the plasma clotting time, a personalized dosage is determined for each patient. This need for monitoring significantly adds to the burden of healthcare cost.

In the last decade new drugs, commonly referred to as direct oral anticoagulants (DOACs), have taken over the prominent place of VKAs in treatment and prevention of thrombosis. After the failed launch of two predecessors, four DOACs have been approved by the U.S. Food and Drug Administration (FDA) and European Medicines Agency (EMA). DOACs are claimed to be more stable than VKAs due to less interactions with food or drugs. Clinical trials have shown that the treatment of venous thromboembolism (VTE) with DOACs without monitoring, and prophylaxis in patients with atrial fibrillation (AF) is not inferior to treatment with VKAs. ${ }^{2-9}$ Consequently, it was determined that routine monitoring can be omitted and dose adjustments are only made in selected cases (e.g. patients with reduced renal/hepatic clearance or extreme body weights). ${ }^{10}$ This became the major selling point of DOACs, because monitoring and personalized dosages can be a burden for patients as well as for physicians. Moreover, omitting visits to specialised anticoagulation clinics could potentially reduce the costs. At the same time, DOACs are currently more expensive as compared to the generic VKA's, such that the total cost for VKA management is still lower than the cost for DOACs without monitoring. Although the real prices of the DOACs are confidential as part of a government negotiated price agreement, most sources estimate the costs of a DOAC around €839per patient per year versus €244for monitored VKA treatment per patient per year in the Netherlands.11-13 On the other hand, a cost-effectiveness study 
which investigated apixaban versus low molecular weight heparin (LMWH)/VKA treatment, showed that treatment with apixaban increases the anticoagulation costs, but decreases the total event-related costs compared to LMWH/VKA treatment (€8178 versus €8414 per treatment per year). ${ }^{14}$

All in all, the question remains if the 'one-size-fits-all' approach is the best way to go when it comes to patient care? Standard dosing goes against the modern trend in many areas of medicine, for example in cancer and cardiovascular medicine, where a development is seen towards personalized medicine. When a modern drug (DOAC) in standard dosage is as good as an old drug (VKAs) in personalized dosage, wouldn't a modern drug in personalized dosages be even better?

During the preparation of this thesis the DOACs have evolved from relatively new and "unknown" drugs to the drugs of choice for patients with VTE as well as AF. Real-life data on the efficiency and safety were not yet available. At first, physicians hesitated to prescribe new drugs without monitoring and some are still tempted by off-label use (i.e. reducing the dose without approved indication). That the effect of DOACs on coagulation cannot be tested at the moment contributes to this problem. Currently, underdosing is a substantial problem that will require further attention in the future, starting by improving the education and awareness of physicians. ${ }^{15}$ Another pitfall of DOAC management is non-adherence. The short half-life of the DOACs comes with the consequence that missing a dose could lead to almost immediate loss of protection against thrombosis. Also in this case, education of the patient, family or other caretakers is important to counter this problem, but assessing DOAC levels could also give more insight in therapy adherence, or drug failure. Furthermore, the rate of clinically relevant bleeding due to oral anticoagulants is still substantial, i.e. 10-17\% per treatment year, which leaves room for improvement in DOAC management. ${ }^{2,3}$ Recently, the question whether or not laboratory monitoring of DOACs is necessary has been debated extensively. This thesis connects to this debate and proposes new ways to measure the effect of DOACs. The topic of this thesis not only concerns an increasingly large population, but is particularly relevant in the light of optimizing DOAC treatment. The research in this thesis was driven by curiosity arising during my clinical residency, in the time that the DOACs were first introduced in the Netherlands. The experiments have been performed in a laboratory with special focus on translational research. In short, it went from bed, to bench and back again. 
With the findings in chapter 2 we show that whole blood thrombin generation (WB TG) and TG might be able to identify patients with a higher risk of bleeding while using VKAs, better than the prothrombin time (PT) from which the international normalized ratio (INR) is calculated. The INR is valuable to determine the current state of (anti)coagulation of a patient, but does not indicate a future bleeding risk. TG by itself, is not able to predict bleeding in individual patients either, but can help to identify patients at risk. The TG parameters could potentially be incorporated in a prediction score. This could ultimately reduce the risk of bleeding complications during treatment.

Chapter 3 investigates the potential benefit of TG in more detail. The limited number of studies that have been performed investigating the potential of TG to detect a bleeding tendency, stresses the need for more research. Interestingly, we found many studies that used TG to investigate the reversibility of DOACs, but no study investigated the relation between TG and the risk of bleeding in patients on DOACs directly. Additionally, TG was used to assess the global coagulation of patients included in some of the large clinical trials, which led to the implementation of DOACs in clinical practice.

There is evidence from the randomized clinical trials with dabigatran and edoxaban, that inter-individual variation in plasma concentrations of DOACs (pharmacokinetics (PK)) relates to outcomes (thrombosis or bleeding) ${ }^{5,16}$; furthermore, Testa et al provided evidence for an association of DOAC levels and thrombotic outcomes ${ }^{17}$, whereas similar associations with peak height and bleeding have been observed in the same observational study (Testa, personal communication 2018). It therefore makes sense that introducing any form of laboratory fine-tuning of DOACs (selecting the right drug and proper dose) will improve DOAC management and reduce complications. The latter would ideally be proven through randomized intervention trials. ${ }^{18}$ DOAC levels can be measured with several newly developed DOAC-specific assays. These are quantitative assays that can reliably measure the amount of DOAC present, but not necessarily the effect of DOACs on coagulation.

In the present thesis the effects of different DOACs on coagulation are investigated in more detail by using more functional assays, in most cases TG. A recently published article has examined the value in determining edoxaban levels versus the effect on coagulation (measured with a newly developed technique), related to clinical outcomes (thrombosis and bleeding). ${ }^{19}$ This paper showed a favourable effect of a functional assay, such as TG. In chapter 5 of this thesis the development of a new assay is described, that can measure rivaroxaban levels as 
well as the effect on coagulation using the same TG set-up. If future research would demonstrate that quantitative and functional measuring of DOACs has a beneficial effect on patient care, the use of such an assay should be considered.

In chapter 6 we have confirmed that in vitro, the response to a fixed dose of rivaroxaban and apixaban on TG varies widely between individuals. Within individuals this variation is less, indicating that the effect of DOACs is quite stable over time. This could lead to the assumption that patients might benefit from initial personalization of the dose, but will not need frequent regular monitoring afterwards. In that case, DOACs (certainly future generic compounds) will remain a more practical and potentially cheaper choice than VKAs.

The question whether high responders will have a higher bleeding risk and low responders will have a higher risk of thrombosis has been addressed in aforementioned trials and observational studies. Critics claim that this evidence cannot be translated to dose adjusted therapy, because fixed doses were studied in clinical trials and consequently doses based on clinical characteristics were registered. Eikelboom and colleagues propose a randomized clinical trial comparing current DOAC policy with laboratory adjusted DOAC dosing. However, in their calculation such study would have immense proportions and would essentially not be feasible. ${ }^{18}$ I would propose that no specific clinical trial is required in order to optimize selection of the proper DOAC for the individual patient, potentially even for guidance of the proper dose. Important is to remain within correct dose ranges, registered for the appropriate indication. At the same time, more evidence would help to support such a strategy and hopefully more studies like the one from Testa et al. will be performed.

An additional aspect to be taken into account is the genetic variation that plays a role in pharmacokinetics and-dynamics. DOACs, studies are limited to dabigatran, showing that genetic variation could lead to higher or lower peak and trough levels, which are associated with an increased risk of bleeding or thrombosis. The (genetic) impact could not only influence drug levels but also functional markers such as TG. From existing literature it is known that the heritability estimate for TG parameters is $\pm 50 \%$, i.e. around $50 \%$ can be contributed to genetic factors. ${ }^{20,21}$ In newly initiated research we have started to investigate to what extent the response to rivaroxaban is genetically determined. Theoretically, in the future, pharmacogenetics could play a role in the decision making process for the determination of the initial dose when prescribing DOACs, redirecting the treatment of thrombosis back towards the paradigm of personalized medicine. 


\section{REFERENCES:}

1. Townsend N, Wilson L, Bhatnagar P, Wickramasinghe K, Rayner M, Nichols M. Cardiovascular disease in Europe: epidemiological update 2016. Eur Heart J. 2016;37(42):3232-3245.

2. Connolly SJ, Ezekowitz MD, Yusuf S, et al. Dabigatran versus warfarin in patients with atrial fibrillation. N Engl J Med. 2009;361(12):1139-1151.

3. Patel MR, Mahaffey KW, Garg J, et al. Rivaroxaban versus warfarin in nonvalvular atrial fibrillation. N Engl J Med. 2011;365(10):883-891.

4. Granger $\mathrm{CB}$, Alexander JH, McMurray JJ, et al. Apixaban versus warfarin in patients with atrial fibrillation. N Engl J Med. 2011;365(11):981-992.

5. Giugliano RP, Ruff CT, Braunwald E, et al. Edoxaban versus warfarin in patients with atrial fibrillation. N Engl J Med. 2013;369(22):2093-2104.

6. Bauersachs R, Berkowitz SD, Brenner B, et al. Oral rivaroxaban for symptomatic venous thromboembolism. N Engl J Med. 2010;363(26):2499-2510.

7. Schulman S, Kearon C, Kakkar AK, et al. Dabigatran versus warfarin in the treatment of acute venous thromboembolism. N Engl J Med. 2009;361(24):2342-2352.

8. Agnelli G, Buller HR, Cohen A, et al. Oral apixaban for the treatment of acute venous thromboembolism. N Engl J Med. 2013;369(9):799-808.

9. Buller HR, Decousus H, Grosso MA, et al. Edoxaban versus warfarin for the treatment of symptomatic venous thromboembolism. N Engl J Med. 2013;369(15):1406-1415

10. Hijazi Z, Hohnloser SH, Oldgren J, et al. Efficacy and safety of dabigatran compared with warfarin in relation to baseline renal function in patients with atrial fibrillation: a RE-LY (Randomized Evaluation of Long-term Anticoagulation Therapy) trial analysis. Circulation. 2014;129(9):961-970.

11. Horizonscan Geneesmiddelen Dabigatran. https://www.horizonscangeneesmiddelen.nl/geneesmiddelen/ dabigatran-etexilaat-cardiovasculaire-aandoeningen-antitrombotische-medicatie/versie2.

12. Horizonscan Geneesmiddelen Rivaroxaban. https://www.horizonscangeneesmiddelen.nl/geneesmiddelen/ rivaroxaban-cardiovasculaire-aandoeningen-antitrombotische-medicatie/versie3.

13. Stichting Farmaceutische Kengetallen.

14. de Jong LA, Dvortsin E, Janssen KJ, Postma MJ. Cost-effectiveness Analysis for Apixaban in the Acute Treatment and Prevention of Venous Thromboembolism in the Netherlands. Clin Ther. 2017;39(2):288-302. e284.

15. Miele C, Taylor M, Shah A. Assessment of Direct Oral Anticoagulant Prescribing and Monitoring Pre- and Post-Implementation of a Pharmacy Protocol at a Community Teaching Hospital. Hosp Pharm. 2017;52(3):207-213.

16. Reilly PA, Lehr T, Haertter $S$, et al. The effect of dabigatran plasma concentrations and patient characteristics on the frequency of ischemic stroke and major bleeding in atrial fibrillation patients: the RE-LY Trial (Randomized Evaluation of Long-Term Anticoagulation Therapy). J Am Coll Cardiol. 2014;63(4):321-328.

17. Testa S, Paoletti $O$, Legnani $C$, et al. Low drug levels and thrombotic complications in high-risk atrial fibrillation patients treated with direct oral anticoagulants. J Thromb Haemost. 2018;16(5):842-848.

18. Eikelboom JW, Quinlan DJ, Hirsh J, Connolly SJ, Weitz JI. Laboratory Monitoring of Non-Vitamin K Antagonist Oral Anticoagulant Use in Patients With Atrial Fibrillation: A Review. JAMA Cardiol. 2017;2(5):566-574.

19. Yin OQP, Antman EM, Braunwald E, et al. Linking Endogenous Factor Xa Activity, a Biologically Relevant Pharmacodynamic Marker, to Edoxaban Plasma Concentrations and Clinical Outcomes in the ENGAGE AF-TIMI 48 Trial. Circulation. 2018;138(18):1963-1973.

20. Martin-Fernandez L, Ziyatdinov A, Carrasco M, et al. Genetic Determinants of Thrombin Generation and Their Relation to Venous Thrombosis: Results from the GAIT-2 Project. PLoS One. 2016;11(1):e0146922.

21. Wichers IM, Tanck MW, Meijers JC, et al. Assessment of coagulation and fibrinolysis in families with unexplained thrombophilia. Thromb Haemost. 2009;101(3):465-470 


\section{ABOUT THE AUTHOR}

Suzanne Zwaveling was born on May $22^{\text {nd }} 1988$ in Utrecht, the Netherlands. She attended the pre-university secondary education at the Praedinius Gymnasium in Groningen and received her degree in 2006. Subsequently, Suzanne started studying Medicine at Maastricht University and received her MD in March 2013. Following this she started a position as a resident internal medicine at the Catharina hospital in Eindhoven. In September 2013 she moved to Curaçao for one year, where she worked as a resident internal medicine in the St. Elisabeth Hospital in Willemstad. After her return to the Netherlands, she worked as a resident internal medicine in the St. Elisabeth hospital in Tilburg. In January 2015 she started her PhD programme named 'The impact of oral anticoagulation on haemostasis - a step toward individualized therapy', at the department of Biochemistry of Maastricht University and partly at Synapse Research Institute, under the supervision of prof. dr. Hugo ten Cate. As of May 2019 Suzanne will start her training in Internal Medicine at the University of Leiden under the supervision of prof. dr. de Feijter. 


\section{LIST OF PUBLICATIONS:}

\section{Publications}

Spronk HM, Cannegieter S, Morange P, Hackeng T, Huisman M, Nagler M, Posthuma J, Ninivaggi M, Zwaveling S, van der Hulle T, Scheres LJ, van Mens TE, Mackman N. Theme 2: Epidemiology, Biomarkers, and Imaging of Venous Thromboembolism (and postthrombotic syndrome). Thromb Res. 2015 Sep;136 Suppl 1:S8-S12.

Vries MJ, Bouman HJ, Olie RH, Veenstra LF, Zwaveling S, Verhezen PW, Ten Cate-Hoek AJ, Ten Cate H, Henskens YM, van der Meijden PE. Determinants of agreement between proposed therapeutic windows of platelet function tests in vulnerable patients. Eur Heart J Cardiovasc Pharmacother. Eur Heart J Cardiovasc Pharmacother. 2017 Jan;3(1):11-17.

Bloemen S*, Zwaveling S*, Ten Cate, H, Ten Cate-Hoek A, de Laat, B. Prediction of bleeding risk in patients taking vitamin $\mathrm{K}$ antagonists using thrombin generation testing. PLoS One. 2017 May 4;12(5):e0176967.

Spronk HMH, Padro T, Siland JE, Prochaska JH, Winters J, van der Wal AC, Posthuma JJ, Lowe G, d'Alessandro E, Wenzel P, Coenen DM, Reitsma PH, Ruf W, van Gorp RH, Koenen RR, Vajen T, Alshaikh NA, Wolberg AS, Macrae FL, Asquith N, Heemskerk J, Heinzmann A, Moorlag M, Mackman N, van der Meijden P, Meijers JCM, Heestermans M, Renné T, Dólleman S, Chayouâ W, Ariëns RAS, Baaten CC, Nagy M, Kuliopulos A, Posma JJ, Harrison P, Vries MJ, Crijns HJGM, Dudink EAMP, Buller HR, Henskens YMC, Själander A, Zwaveling S, Erküner O, Eikelboom JW, Gulpen A, Peeters FECM, Douxfils J, Olie RH, Baglin T, Leader A, Schotten U, Scaf B, van Beusekom HMM, Mosnier LO, van der Vorm L, Declerck P, Visser M, Dippel DWJ, Strijbis VJ, Pertiwi K, Ten Cate-Hoek AJ, Ten Cate H. Atherothrombosis and Thromboembolism: Position Paper from the Second Maastricht Consensus Conference on Thrombosis. Thromb Haemost. 2018 Feb;118(2):229-250.

Zwaveling S, Bloemen S, de Laat B, Ten Cate H, Ten Cate-Hoek AJ. Calibrated automated thrombinography (CAT), a tool to identify patients at risk of bleeding during anticoagulant therapy? A systematic review. TH open 2018;2:e291-e302. 
Bloemen S, Zwaveling S, Douxfils J, Roest M, Kremers R, Mullier F. The anticoagulant effect of dabigatran is reflected in the lag time and time-to-peak, but not in the endogenous thrombin potential or peak, of thrombin generation. Thromb Res. 2018 Nov;171:160-166.

Bloemen S*, Zwaveling S*, Douxfils J, Peters T, Wagenvoord R, Hemker HC, Mullier F, de Laat B, Roest M, Kremers R. A new assay for the concomitant assessment of rivaroxaban concentration and its impact on thrombin generation. - Submitted at Clinical Chemistry

Zwaveling S, Konings J, Kelchtermans H, Hemker HC, Bloemen S. The variability in response to rivaroxaban and apixban between and within individuals, over time. - To be submitted

Zwaveling S, Konings J, Miszta A, Hemker HC, de Groot PH, Bloemen S. The differential effects of dabigatran, rivaroxaban and apixaban on fibrinolysis. - To be submitted

Kicken CH, Van der Vorm LN, Zwaveling S, Schoenmaker E, Remijn, JA, Huskens D, De Laat B. A hypoxic environment attenuates exercise-induced procoagulant changes due to decreased platelet activation. - Submitted 


\section{Oral Presentations:}

Zwaveling S, Bloemen S, Konings J, Kelchtermans H, Hemker HC, Miszta A, de Laat B. Differential effects of dabigatran and rivaroxaban on fibrinolysis. European and Mediterranean League against Thrombotic Diseases (EMLTD), Venetië 2018, trainee travel award

Zwaveling S, Bloemen S, Konings J, Kelchtermans H, Hemker HC, Miszta A, de Laat B. The Effects of DOACs on fibrinolysis. International Society for Laboratory Hematology (ISLH), Brussel 2018, trainee travel award

\section{Zwaveling S}

Antistolling, nooit meer prikken alleen nog slikken, direct oral anticoagulants, ambassadeursdag Trombosestichting Nederland, Utrecht 2017

\section{Zwaveling S}

Wat is trombose? Wereld Trombosedag, MUMC+, Maastricht 2015

Zwaveling S, Vroomen L, Roos A, Bindels A.

Standaard doseren van LMWH o.b.v. opnamegewicht leidt tot subtherapeutische anti-factor Xa levels in ICU patiënten. Nederlands internistendagen (NIV), MECC, Maastricht 2013

\section{Awards:}

- Young investigator award ISTH 2018, Dublin, Ireland

- Trainee travel award EMLTD, 2018 Venice, Italy

- Trainee travel award ISLH 2018, Brussels, Belgium

- 1st place at the postercompetition Santeon wetenschapsdagen, Catherina ziekenhuis, 2013, Eindhoven, the Netherlands 


\section{DANKWOORD}

Promoveren was mij nooit gelukt zonder de inzet en hulp van collega's, familie en vrienden. In dit hoofdstuk wil ik graag iedereen bedanken die heeft bijgedragen aan dit proefschrift.

Geachte prof. dr. ten Cate, beste Hugo, dank je voor de kans om bij jou te mogen promoveren. Ik heb veel ontzag voor je rol in het translationele onderzoek. Jouw hartstocht hiervoor is tastbaar en aanstekelijk. Veel van mijn voorgangers noemen je een baken van rust, maar dat zal ik hier niet schrijven. Rustig waren de afgelopen 4 jaar in ieder geval niet. In de tijd dat er grote veranderingen optreden in de wereld van antistolling ben je niet bang om je mening te geven, ook al druist deze in tegen de algemene opinie. Ik vond het inspirerend om je te horen spreken op congressen. Dank voor je hulp bij projecten, het meedenken, het snelle nakijken van mijn stukken, je humor, het feit dat je deur altijd openstaat (ook als hij dicht is) Ik weet tot op heden niet wat je over mij heb geschreven in de aanbevelingsbrief voor mijn sollicitatie voor de opleiding, maar het heeft geleid tot een positief resultaat, waarvoor dank! Ik zal onze tijd op D5 niet snel vergeten.

Beste dr. Bloemen, lieve Saartje, wie had van te voren gedacht dat wij samen zo'n goed team zouden zijn? Mijn geboren tegenpool! Ik heb erg veel respect voor je Vlaamse nuchterheid, je perfectionisme, je voorspellende blik en vooral je onuitputtelijke doorzettingsvermogen en strijdkracht. In het lab ben jij een machine! Als bij jou eenmaal het idee voor een bepaald project viel, dan werd dat het liefst diezelfde dag nog uitgevoerd. Waarom iets morgen doen, als het ook gisteren kon? Als het maar voor 16:00 uur gebeurd is... Onderzoek doen ging bij ons razendsnel. Dank je, dat je mij, als verdwaalde arts in het lab zonder ervaring in het handteren van een pipet of enig besef van de lab-mores, toch onder je hoede hebt genomen, en van mij een basale onderzoeker hebt gemaakt. We hebben heel veel samen meegemaakt in de afgelopen 4 jaar, en je bent voor mij onmisbaar geweest tot het einde. Ondanks je befaamde uitspraken "tjah, het is wat het is...", en "de data zijn de data...", heb ik veel plezier geput uit onze projecten, uit onze tijd in het lab en uit ons "congressenrondje" afgelopen jaar. Dank voor je vertrouwen in mij. Je hebt mij laten zien dat basaal onderzoek doen super leuk kan zijn.

Beste dr. ten Cate-Hoek, beste Arina, dank je voor de intensieve begeleiding met name in het eerste jaar. Deze periode is voor mij zeer leerzaam geweest. Je hebt me laten zien hoe je 
"systematisch" (dat wil zeggen stap voor stap) een "systematic review" schrijft; en bovendien dat je meer kans hebt om je promotie succesvol af te ronden als je een Excel-sheet hebt.

Geachte leescommissie, prof. dr. Hackeng, prof. dr. Ariëns, dr. Winckers, prof. dr. Schutgens en prof. dr. Koopmans, hartelijk dank dat jullie de tijd hebben genomen om mijn proefschrift te lezen en te beoordelen.

Prof. dr. Hackeng, beste Tilman, wat een feest was het met jou als mentor! Dank voor alle adviezen, je altijd goede stemming, je kordaatheid, je straight-forward visie en je goede smaak in wijn! Je zorgt erg goed voor je afdeling, onder andere met goed verzorgde borrels.

Prof. dr. Hemker, ik vond het een eer om met u te mogen werken en van u de hardcore biochemie en kinetiek te leren, de achtergrond van de TG. Ontzettend bedankt voor uw mooie illustraties en de leuke brainstorm-momenten over gepaste en minder gepaste stellingen.

Dear co-authors, it has been a pleasure to work with you. Your suggestions have improved the quality of my work greatly. A special thanks to dr. Jonathan Douxfils and prof. dr. François Mullier from Namur for their extra effort and critical comments that improved several articles of this thesis. You have been a source of inspiration and I look forward to your work on DOAC measurements in the future.

Beste Henri, René, Paola, José, Renske, Avi, Yvonne, Marieke, Rick, Paul, Elisa, Billy, Simone en andere leden van de groep 'Clinical Thrombosis van Haemostasis' a.k.a. 'de Hugo-groep'. Dank voor de onmisbare, leerzame discussies op dinsdagochtend! Het belang om met meerdere kritische blikken en kennis vanuit verschillende achtergronden naar data te kijken, is niet te onderschatten en ook best leuk! Daarnaast was er ook ruimte voor gezelligheid en erg veel taart (zelfgebakken of door Lemmens). Speciale dank gaat naar mijn kamergenootjes. Minka, mijn grote voorbeeld (op meer manieren dan je denkt)! Dank je voor het altijd tonen van de juiste weg, je kennis, je enorme geduld en je lieve meelevende karakter. Ik hoop dat we vriendinnen en collega's interne blijven in de toekomst! Jens, dank voor je relativerend vermogen en je altijd positieve kijk op het leven, ik wens je heel veel succes met je eigen promotie! Jelle, bedankt voor het altijd hooghouden van sfeer, met name tijdens de uitjes. Elham, wat fijn dat je promotie zo goed is gegaan! Anouk, ik wens je ook heel veel succes met promoveren. 
Andere collega's binnen de vakgroep biochemie: dank voor al jullie input op de woensdagochtend besprekingen, de vele afdelingsborrels, uitjes en de gezelligheid tijdens de congressen. Elisabetta en Stella, jullie zijn van die onmisbare karakters die deze afdeling zo speciaal maken. Trees en Lidewij, jullie fantastische vermogen om altijd een gaatje te kunnen vinden in overvolle agenda's van veel te drukke professoren, en de hulp bij de logistiek omtrent promoveren wordt heel erg op prijs gesteld. En dan natuurlijk mijn mede-promovendi, in het bijzonder Rick, Armand, Daniëlle, Annemiek, Danique, Bogac, Frauke en Peter, het leven van een PhD gaat niet altijd over rozen, ontzettend fijn om met jullie te kunnen lachen om ons lot. Stijn, mijn maatje op biochemie vanaf (voor mij) het begin! Met veel plezier denk ik terug aan o.a. de CARIM-caterpillars, de NVTH's, en onze eindeloze gesprekken over de toekomst (vooral de jouwe) onder het genot van koffie/choco om stipt 15:30 uur. Ik heb heel veel bewondering voor je! Hoe kun je elke dag om 16:00 uur naar huis gaan om vervolgens cum laude te promoveren? Daarnaast lach ik me kapot om je stiptheid en je vermogen om alles te overdenken. Fijn dat je blijft, je hoort hier thuis!

Het gehele team achter de plaatjespoli: Minka, Renske, Hugo, Yvonne, Rick, Paul, Mariëlle, Carol, Daniëlle, Linda, Bart, Jos en René. Dankzij jullie is deze studie van de grond gekomen en worden alle experimenten zorgvuldig uitgevoerd. Voor mij was het fijn om deel uit te maken van deze klinische studie om zo het contact met de kliniek niet te verliezen. Renkse en Hugo, dank voor jullie begeleiding. WESP-studenten waaronder oa. Robin, Rachelle, Lian, Eline, Merel, Sanne, Bregje en Charlotte, dankzij jullie had ik het laatste jaar meer tijd om aan de thesis te schrijven, mijn dank hiervoor is groot.

Collega's van Synapse, in het bijzonder Joke, Dana, Hilde, Erik, Iris, Cui en mijn fellow-PhD's: Jun, Yaqiu, Dongmei, Walid and Lisa. Thanks for the cooperation and your contribution to a good atmosphere in the lab. I wish you all the best of luck for the future.

Tijdens mijn promotie heb ik met veel plezier enkele studenten mogen begeleiden, waar ik zelf ook veel van geleerd heb. Daarvoor dank aan Alicia, Evi, Jaël en Charlotte.

Alle medewerkers van de trombosedienst Maastricht, in het bijzonder Jolanda, Loes, Maurice, Marie-Jose, Cissi, Karly en Arina. Dank jullie voor de fijne samenwerking, ik wens jullie veel goeds toe in de toekomst. 
Veel dank ook aan alle vrijwilligers en patiënten die zichzelf beschikbaar hebben gesteld en daardoor een grote rol vervullen voor dit proefschrift.

Dank aan alle mensen die financieel hebben bijgedragen aan deze dag.

Alexandra, Annelijn, Anneloes, Anouk, Camille, Eline, Jessica, Maaike, Marin, Marloes, Romy en Simone dank voor onze vriendschap en jullie steun, ik heb ontzettend veel zin in de volgende reis!

Dushi Korsou, waar het voor mij allemaal is begonnen. Ils, Sammie, Rosa, Eva, Jan, Greet, Bart, Floor, Mahsa en Wertz. Dank voor de fantastische tijd samen, het was ontzettend leuk om met jullie samen te werken en ik geniet nog steeds van onze reünies. Ik hoop dat deze dag weer zo'n gelegenheid mag zijn. Beste Ken, lieve Jeanne, zonder jullie vertrouwen in mij zou ik hier nu niet staan. Dankzij jullie kennis, inzicht en vaardigheden ben ik een betere dokter geworden.

Mijn collega's van de Interne/MDL in Maastricht, dank voor de fijne samenwerking op D5. Daarnaast, veel dank dat ik mee mocht op skireis, het was echt onvergetelijk!

Prof. dr. de Feijter en dr. Koster, dank voor het vertrouwen in mij als assistent interne geneeskunde in opleiding. Ik heb erg veel zin om te starten in het Groene Hart Ziekenhuis.

Wouter en Jan, sporten en zingen met jullie was een onmisbare uitlaatklep. Van jullie heb ik geleerd hoe belangrijk het is om hiervoor tijd vrij te maken, hoe druk het ook is. Ik zal hieraan blijven denken in de toekomst.

Lieve Viola, Cecile en Martijn, 'the hard core'! Wat een tijd hebben wij achter de rug! Ik denk niet dat ik de afgelopen 4 jaar was doorgekomen zonder jullie aan mijn zijde. Jullie hebben het in ieder geval zo veel leuker gemaakt! We hebben een hele mooie vriendschap opgebouwd op een voor mij onverwachte plek, waarvan ik hoop dat hij nog lang stand zal houden. Lieve Viool, jouw vermogen om elke situatie uit te kunnen drukken in een grappige afbeelding is buitengewoon. Lieve Ciel, ondanks dat we in sommige dingen heel erg verschillen, waardeer ik jou enorm! Dank voor je vermogen om alles tot een (enigszins geëscaleerd) feestje te maken! Lieve Mart, dank voor het feit dat je geen navigatie-skills hebt. Het gaat om 
reis, niet om de bestemming. Dank je wel dat je mijn paranimf wilt zijn. Jullie waren er altijd voor mij, door dik en dun. We stonden voor vuren en afgronden, maar kwamen er samen altijd door. Dank voor al jullie bijdragen aan dit proefschrift, jullie kritische blikken, Mart's poëtische Engels, Ciel's grammarnazi skills, maar met name ook dank voor alles wat matig heeft bijgedragen aan dit proefschrift: de feestjes, picknicks, fun valley's, congressen, liters wijn en (speciaal) bier, films, veggy risotto's, spoedbijeenkomsten in café's en bij ons thuis en natuurlijk de memorabele trips. Hopelijk komt die surf/chill vakantie er binnenkort.

Lieve Eem en Cath, dank voor jullie steun en vertrouwen in mij, jullie wijze raad en natuurlijk voor jullie onvoorwaardelijke vriendschap al sinds ik me kan herinneren. Ik zal nooit vergeten dat we alle drie in dezelfde maand een nieuwe baan (of promotie) kregen en dit samen konden vieren. Lieve Juud en Sas ook jullie bedankt voor jullie vriendschap en de moeite om elke keer maar weer naar Maastricht te komen. Ik heb heel veel zin om naar jullie toe te verhuizen, zodat we wat vaker bij elkaar op bezoek kunnen gaan.

Lieve familie Pijning, Henk, Julia, Aster, Anna en Leon en natuurlijk Oma. Hoe vaak was ik bijna in het schuurtje beland? Bedankt voor jullie betrokkenheid en oprechte interesse in mijn onderzoek. Bedankt voor jullie luisterend oor, ook als de interesse echt niet meer op te brengen was. Ik hoop dat ik deze dag samen met jullie kan vieren.

Ook de familie Zwaveling, in het bijzonder Opa Albert en Oma Mieke, bedankt voor het goede voorbeeld, ik hoop dat jullie er vandaag bij kunnen zijn in het verre Maastricht.

Lieve Bianca, Rob, Emma en Max, ook jullie bedankt voor jullie blind vertrouwen in mij en de steun die ik zomaar onvoorwaardelijk kreeg; ook voor jullie geduld in de tijd dat ik met mijn laptop aan tafel zat.

Lieve Em, mijn grote kleine zus. Wat ben ik trost op jou! Ik heb grote bewondering voor hoe jij je hebt ontwikkeld de laatste jaren en er lijkt geen einde aan te komen. We hebben nog lang niet alles van jou gezien! Samen met Justin ga je nog zoveel moois tegemoet! Je bent voor mij een grote steun geweest tijdens dit gehele traject en ik vindt het ontzettend fijn dat je deze dag naast me staat. 
Lieve Sooph, mijn kleine grote zus! Hoe eenzaam zou ik zijn als jij mij niet af toe belde. Er zijn weinig mensen met wie ik zoveel kan lachen als met jou. Bovendien kwam jouw uitvinding van "het Slaventeam" af en toe goed van pas. Multiple inzetbaar en faalt nooit. Ook voor jou heb ik grote bewondering, met name hoe jij en Michael samen door het leven gaan. Ik hoop heel erg dat je komend jaar nog even in Nederland blijft hangen. Special thanks to you and Michael for creating the cover of my thesis and also for creating "chillie". You were always the creative one...

Lieve papa en mama, ik ben eruit! We hebben een vrij bijzonder, maar toch het allerleukste gezin en ik kan me echt niets beters wensen. Ontzettend bedankt voor jullie onvoorwaardelijke steun en het oprechte vertrouwen dat jullie hebben in mij, maar ook in Emma en Sophie.

Liefste Jor, zonder jou was dit nooit gelukt. Dank je wel voor je onvoorwaardelijke liefde, steun, begrip en geduld. Dank je voor de vanzelfsprekendheid waarmee wij er voor elkaar zijn; dat jij me opvangt als het te laat is geworden, dat je me naar buiten trekt in het kader van het Suus-en-Jor-fitheids-programma en voor al die duizend andere dingen. Met name wil ik je bedanken voor onze fantastische tijd in Nieuw Zeeland. lets wat we (jij) altijd al wilden en een belangrijke break in mijn promotie traject. Deze reis was onvergetelijk. Wat hebben we genoten van het land, de natuur, het reizen en het sporten. Ik kan niet wachten om samen met jou te verhuizen naar misschien wel ons eerste echte huis! Ik houd ontzettend veel van jou. Op naar een nieuw avontuur! 